\title{
FORCE-REFLECTING TELEOPERATION OVER WIDE-AREA NETWORKS
}

\author{
by \\ Ilia G. Polouchine \\ Candidate of Technical Sciences, M. Sc.
}

A Thesis submitted to the Faculty of Graduate Studies and Research in partial fulfillment of the requirements for the degree of Doctor of Philosophy in Electrical Engineering

Ottawa-Carleton Institute for Electrical and Computer Engineering Department of Systems and Computer Engineering Carleton University, Ottawa, Ontario, Canada

Copyright (CIlia G. Polouchine, 2008 


$\begin{array}{ll}\begin{array}{l}\text { Library and } \\ \text { Archives Canada }\end{array} & \begin{array}{l}\text { Bibliothèque et } \\ \text { Archives Canada }\end{array} \\ \begin{array}{l}\text { Published Heritage } \\ \text { Branch }\end{array} & \begin{array}{l}\text { Direction du } \\ \text { Patrimoine de l'édition }\end{array} \\ \begin{array}{l}\text { 395 Wellington Street } \\ \text { Ottawa ON K1A 0N4 } \\ \text { Canada }\end{array} & \begin{array}{l}\text { 395, rue Wellington } \\ \text { Ottawa ON K1A 0N4 } \\ \text { Canada }\end{array}\end{array}$

Your file Votre référence ISBN: 978-0-494-47487-7 Our file Notre référence ISBN: 978-0-494-47487-7

NOTICE:

The author has granted a nonexclusive license allowing Library and Archives Canada to reproduce, publish, archive, preserve, conserve, communicate to the public by telecommunication or on the Internet, loan, distribute and sell theses worldwide, for commercial or noncommercial purposes, in microform, paper, electronic and/or any other formats.

The author retains copyright ownership and moral rights in this thesis. Neither the thesis nor substantial extracts from it may be printed or otherwise reproduced without the author's permission.
AVIS:

L'auteur a accordé une licence non exclusive permettant à la Bibliothèque et Archives Canada de reproduire, publier, archiver, sauvegarder, conserver, transmettre au public par télécommunication ou par l'Internet, prêter, distribuer et vendre des thèses partout dans le monde, à des fins commerciales ou autres, sur support microforme, papier, électronique et/ou autres formats.

L'auteur conserve la propriété du droit d'auteur et des droits moraux qui protège cette thèse. $\mathrm{Ni}$ la thèse ni des extraits substantiels de celle-ci ne doivent être imprimés ou autrement reproduits sans son autorisation.
In compliance with the Canadian Privacy Act some supporting forms may have been removed from this thesis.

While these forms may be included in the document page count, their removal does not represent any loss of content from the thesis.
Conformément à la loi canadienne sur la protection de la vie privée, quelques formulaires secondaires ont été enlevés de cette thèse.

Bien que ces formulaires aient inclus dans la pagination, il n'y aura aucun contenu manquant.

\section{Canada}




\section{Abstract}

Bilateral (force-reflecting) teleoperation in the presence of network induced communication constraints presents significant challenges in terms of stability and transparency of the teleoperator system. It is well-known that the stability of the force reflecting teleoperators is compromised in the presence of even small communication delays. Also, stability and transparency are conflicting goals; in particular, high force reflection gain provides a better kinesthetic as well as tactile feedback, however, it also destabilizes the overall system due to increasing the closed-loop gain. In this thesis, a set of results is presented towards stable and transparent force-reflecting teleoperation in the presence of communication constraints typical for serial communication networks. A small gain approach to network-based bilateral teleoperation is systematically developed. The approach is built upon a new version of the input-to-output stability small gain theorem for systems which communicate over multiple networked channels. Based on this theorem, schemes for bilateral teleoperation over networks are developed that guarantee the stability/tracking properties in the presence of network induced communication constraints. Projection-based force reflection algorithms are introduced that solve the contradiction between stability, maneuvrability, and high force reflection gain; in particular, these algorithms allow for achieving the stability for arbitrarily low damping of the master manipulator and arbitrarily high force reflection gain Next, the problem of design of the network-based teleoperators enhanced by means of virtual environment is addressed. The approach proposed is based on a nonlinear sampled-data design framework, and uses ideas from model-based control as well as multi-rate sampled data systems. The control algorithms and the communication protocols are presented that guarantee stability of the overall system under mild assumptions imposed on communication process. 


\section{Acknowledgements}

I would like to express my sincere gratitude to my supervisors Dr. Peter X. Liu and Dr. Chung-Horng Lung for giving me the opportunity to work on the topic of teleoperation and for their constant support and most helpful advice throughout my doctoral studies. I'm thankful to Dr. Rafik A. Goubran and Dr. Jérôme Talim from the Department of Systems and Computer Engineering, Dr. Mojtaba Ahmadi from the Department of Mechanical and Aerospace Engineering, Dr. Shervin Shirmohammadi from the School of Information Technology and Engineering at the University of Ottawa, and Dr. Guangjun Liu from the Department of Aerospace Engineering at Ryerson University, for their willingness to serve as committee members. I'd also like to thank the helpful and considerate administrative and technical staff members from the Department of Systems and Computer Engineering, as well as many friends and fellow graduate students.

The financial support of Natural Sciences and Engineering Research Council of Canada and Carleton University is gratefully acknowledged. 


\section{Contents}

1 Introduction 1

1.1 Master-Slave Teleoperator Systems and Their Applications to Surgery 2

1.1.1 Remote Surgery . . . . . . . . . . . . . . 3

1.1.2 Micro-Surgery: Scaled Teleoperation .............. 4

1.1.3 Minimally-Invasive Surgery . . . . . . . . . . . . . . 4

1.2 Importance of The Force Feedback . . . . . . . . . . . . . . 5

1.3 Transparency and Fidelity . . . . . . . . . . . . . 7

1.4 Stability vs. Transparency Trade-Off . . . . . . . . . . . . . 8

1.5 The Effect of Communication Delay . . . . . . . . . . . . 10

1.5.1 The Effect of Time-Delay on Performance of Telesurgery ...................... 11

1.5.2 Transparency in The Presence of Time Delay . . . . . . . 12

1.5.3 Stability in The Presence of Time Delay . . . . . . . . . . . . 12

1.6 Design Guidelines for Telesurgery Systems . . . . . . . . . . . 14

1.7 Thesis Contributions . . . . . . . . . . . . . . 15

1.8 Thesis Structure . . . . . . . . . . . . . . . 17

2 Small-Gain Approach to Network-Based Teleoperation 20

2.1 Introduction: Stability and Small Gain Theorems . . . . . . . . . 21 
2.2 Features of the Network-Based Teleoperators . . . . . . . . . . 23

2.2 .1 Sampled-Data Implementation . . . . . . . . . . . . . 23

2.2 .2 Multiple Channels . . . . . . . . . . . . . . . . 24

2.2 .3 Communication Constraints . . . . . . . . . . . . . . 24

2.3 Technical Preliminaries $\ldots \ldots \ldots \ldots \ldots \ldots \ldots$

2.3.1 Basic Notation and Definitions $\ldots \ldots \ldots \ldots . \ldots 25$

2.3.2 Multidimensional Extensions of $\mathcal{G}, \mathcal{K}, \mathcal{K}_{\infty}$-Classes $\ldots \ldots .25$

2.3.3 Input-to-State Stability and Input-to-Output Stability of FunctionalDifferential Equations $\ldots \ldots \ldots \ldots \ldots$

2.4 Network-Based Interconnections . . . . . . . . . . . . . 28

2.5 Small-Gain Theorem . . . . . . . . . . . . . . . 33

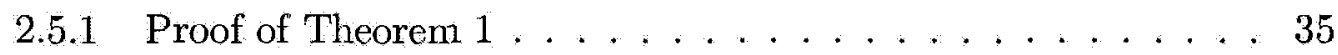

2.6 Example . . . . . . . . . . . . . . . . 38

2.7 Conclusion . . . . . . . . . . . . . . . . . . 40

3 Small-Gain Based Schemes for Stable Bilateral Teleoperation 41

3.1 Introduction . . . . . . . . . . . . . . . . . . . . . 41

3.2 Mathematical Model of The Teleoperator

System . . . . . . . . . . . . . . . . . . 42

3.2.1 Master and Slave Manipulators . . . . . . . . . . . 42

3.2 .2 Communication Constraints . . . . . . . . . . 43

3.3 Control Schemes $\ldots \ldots \ldots \ldots \ldots$. . . . . . . . . 44

3.4 Force Reflection . . . . . . . . . . . . . . . . . 45

3.5 Environmental Dynamics . . . . . . . . . . . . . . 47

3.6 Stability . . . . . . . . . . . . . . . 48

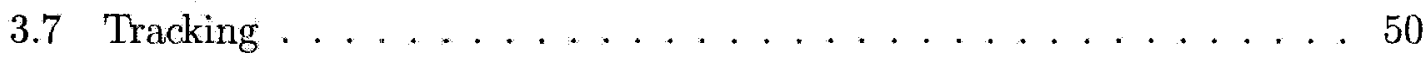

3.8 Proofs $\ldots \ldots \ldots \ldots \ldots \ldots \ldots \ldots \ldots \ldots \ldots \ldots$ 
3.9 Experimental Results . . . . . . . . . . . . . . . . 60 60

4 Projection-Based Force Reflection Algorithms $\quad 74$

4.1 Introduction . . . . . . . . . . . . . . . . 74

4.2 Trade-Off Between Stability and High Force Reflection Gain . . . . . 75

4.3 Projection-Based Force Reflection Algorithms . . . . . . . . . . . . . 78

4.4 General Stability Result . . . . . . . . . . . . . . . . 80

4.5 A Scheme with Direct Human Force Measurement . . . . . . . . . 86

4.6 A Scheme without Human Force Measurement . . . . . . . . . . . . 88

4.7 Experimental Results . . . . . . . . . . . . . . . . 90

4.8 Conclusions . . . . . . . . . . . . . . . . 100

5 Model-Based Teleoperation with Communication Constraints 101

5.1 Introduction . . . . . . . . . . . . . . . 101

5.2 Model-Based Approach to Nonlinear

Networked Control Systerns . . . . . . . . . . . . . . . . 103

5.2.1 Sampled-Data Stabilization of Nonlinear Systems . . . . . . 106

5.2 .2 Protocol for Model-Based NCS . . . . . . . . . . . . 110

5.2.3 Stability and Performance Recovery . . . . . . . . . . . 114

5.3 Virtual Reality Enhanced Bilateral Teleoperation with Communication Constraints . . . . . . . . . . . . . . . . . . 121

5.3.1 Approximate Discrete-Time Model Based Design ... . . . . 122

5.3 .2 Proof of Theorem $9 \ldots \ldots \ldots . \ldots \ldots$

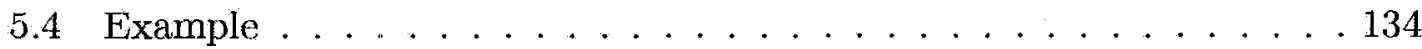

5.5 Conclusions . . . . . . . . . . . . . . . . . . . . 140

6 Concluding Remarks $\quad 143$

6.1 Thesis Contributions ...................... 143 
6.2 Manuscripts Published and Submitted . . . . . . . . . 145

6.3 Possible Directions for Future Research . . . . . . . . . . . . . 148

$\begin{array}{ll}\text { References } & 151\end{array}$

A Modelling of the Phantom TM Device $\quad 167$

A.1 Forward Kinematics . . . . . . . . . . . . . . 167

A.2 Manipulator Jacobian . . . . . . . . . . . . . . . 172

A.3 Dynamics . . . . . . . . . . . . . . . . . . . 174

A.4 Parameter Identification . . . . . . . . . . . . . . . . 180

B Communication Aspects of Telesurgery Over The Internet 188

B.1 Visual and Haptic Feedback in Telesurgery . . . . . . . . . . . 189

B.2 Comparison of QoS Requirements for Visual and Haptic Data . . . 190

B.3 Protocols for Haptic Communication in the Internet . . . . . . . . 191

B.3.1 TCP and UDP . . . . . . . . . . . . . . . . . . 191

B.3.2 Example: TeXCP for Haptic Data . . . . . . . . . . . . . 193

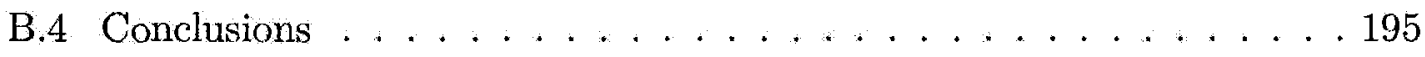




\section{List of Figures}

2.1 Network-based interconnection . . . . . . . . . . . . . . . . 29

3.1 Experimental setup . . . . . . . . . . . . . . 60

3.2 Example of the master trajectory generated by the human operator . 65

3.3 Example of communication delay function, $\tau(\cdot) \sim \mathbb{N}\left(T_{a v}, \sigma^{2}\right), T_{a v}=$

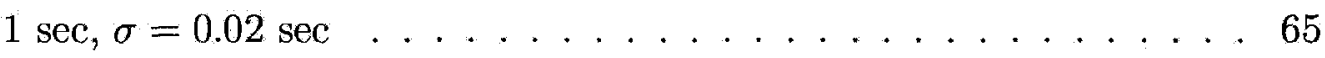

3.4 Teleoperator system response, Delay $\approx 0, \mathrm{FR}$ gain $=500: \mathrm{X}$-trajectories, master and slave (top); Contact X-Forces (bottom) . . . . . . . 66

3.5 Teleoperator system response, Delay $\approx 0, \mathrm{FR}$ gain $=1500: \mathrm{X}$-trajectories, master and slave (top); Contact X-Forces (bottom) . . . . . . . 67

3.6 Teleoperator system response, Delay $\approx 0, \mathrm{FR}$ gain $=3000$ : $\mathrm{X}$-trajectories, master and slave (top); Contact X-Forces (bottom) . . . . . . . . 68

3.7 Teleoperator system response, Delay $\approx 0, \mathrm{FR}$ gain $=4000: \mathrm{X}$-trajectories, master and slave (top); Contact X-Forces (bottom) . . . . . . . 69

3.8 Teleoperator system response, Delays $\tau_{f}(\cdot), \tau_{b}(\cdot) \sim \mathbb{N}\left(T_{a v}, \sigma^{2}\right), T_{a v}=$ $1 \mathrm{~s}, \sigma=0.02 \mathrm{~s}$, FR gain=500: X-trajectories, master and slave (top); Contact X-Forces (bottom) . . . . . . . . . . . . . . . 70

3.9 Teleoperator system response, Delays $\tau_{f}(\cdot), \tau_{b}(\cdot) \sim \mathbb{N}\left(T_{a v}, \sigma^{2}\right), T_{a v}=$ $1 \mathrm{~s}, \sigma=0.02 \mathrm{~s}$, FR gain=1000: X-trajectories, master and slave (top); Contact X-Forces (bottom) . . . . . . . . . . . . . . . 71 
3.10 Teleoperator system response, Delays $\tau_{f}(\cdot), \tau_{b}(\cdot) \sim \mathbb{N}\left(T_{a v}, \sigma^{2}\right), T_{a v}=$ $1 \mathrm{~s}, \sigma=0.02 \mathrm{~s}$, FR gain=1500: X-trajectories, master and slave (top); Contact X-Forces (bottom) . . . . . . . . . . . . 72

3.11 Teleoperator system response, Delays $\tau_{f}(\cdot), \tau_{b}(\cdot) \sim \mathbb{N}\left(T_{a v}, \sigma^{2}\right), T_{a v}=$ $2.5 \mathrm{~s}, \sigma=0.05 \mathrm{~s}, \mathrm{FR}$ gain=1000: X-trajectories, master and slave (top); Contact X-Forces (bottom) ............. 73

4.1 Teleoperator system response, Delay $\approx 0, \mathrm{FR}$ gain $=4000, \alpha=1$ (i.e., direct force reflection), free motion: X-trajectories, master and slave (top); Contact X-Forces (bottom) . . . . . . . . . . . 93

4.2 Teleoperator system response, Delay $\approx 0, \mathrm{FR}$ gain $=4000, \alpha=0.5$, free motion: X-trajectories, master and slave (top); Contact X-Forces

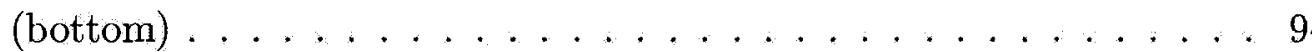

4.3 Teleoperator system response, Delay $\approx 0, \mathrm{FR}$ gain $=4000, \alpha=0.5$, operator holds: X-trajectories, master and slave (top); Contact XForces vs. Reflected X-Forces(bottom) . . . . . . . . . . . . . 95

4.4 Teleoperator system response, Delay $\approx 0, \mathrm{FR}$ gain $=4000, \alpha=0.25$, free motion: X-trajectories, master and slave (top); Contact X-Forces

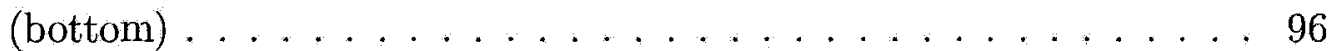

4.5 Teleoperator system response, Delay $\approx 0, F R$ gain $=4000, \alpha=0.25$, operator holds: X-trajectories, master and slave (top); Contact XForces vs. Reflected X-Forces(bottom) . . . . . . . . . . . 97

4.6 Teleoperator system response, Delay $\approx 2$ sec, FR gain $=4000, \alpha=0.3$, free motion: X-trajectories, master and slave (top); Contact X-Forces

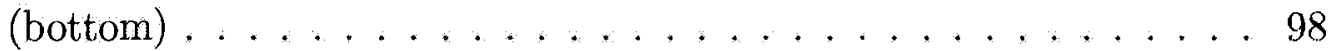


4.7 Teleoperator system response, Delay $\approx 2 \mathrm{sec}, \mathrm{FR}$ gain $=4000, \alpha=0.3$, operator holds: X-trajectories, master and slave (top); Contact XForces vs. Reflected X-Forces(bottom) . . . . . . . . . . . . 99

5.1 Structure of VE-enhanced teleoperator system . . . . . . . . . . 121

5.2 Kinematic Scheme . . . . . . . . . . . . . . . . . 134

5.3 Reference trajectories: joint space (top) and task space(bottom) . . . 136

5.4 Slave manipulator interconnected with environment . . . . . . . . 138

5.5 Direct force feedback: X-trajectories, master and slave devices . . . . 139

5.6 VE based force feedback: X-trajectories, master, slave and virtual slave (top); X-components of the contact forces, environment and virtual environment (bottom) . . . . . . . . . . . . . 141

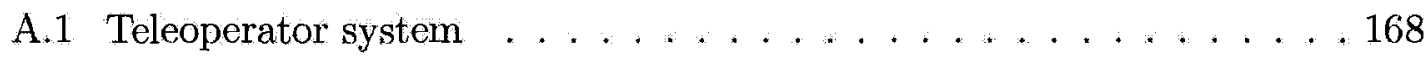

A.2 Master device ... . . . . . . . . . . . . . . 168

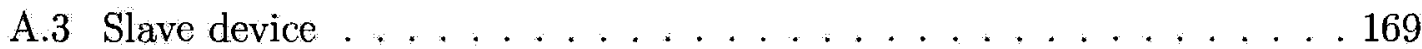

A.4 Kinematic scheme of the Phantom ${ }^{T M}$ device . . . . . . . . . 169 


\section{List of Acronyms}

FDEs Functional-Differential Equations

FR Force Reflection

IOS Input-to-Output Stability

ISS Input-to-State Stability

ODEs Ordinary Differential Equations

RTT Round Trip Time

UDP User Datagram Protocol 


\section{Chapter 1}

\section{Introduction}

In this chapter, some introductory material is presented that is related to masterslave teleoperator systems with special emphasis to surgical applications. At the end of this chapter, the goals of this thesis are identified and discussed. The chapter is organized as follows. In Section 1.1, we introduce master-slave force-reflecting teleoperator systems and discuss three conceptually different applications of the master-slave teleoperator systems to surgery: remote surgery, micro-surgery, and minimally invasive surgery. In Section 1.2 , we give a brief survey of the experimental results that demonstrate importance of the haptic feedback in telesurgery. Notions of transparency and fidelity of teleoperation are discussed in Section 1.3. In Section 1.4, a trade-off between transparency and stability in teleoperator systems is briefly addressed. Section 1.5 describes the effect of communication delay on stability and performance of teleoperation. General design requirements for telesurgery systems are summarized in Section 1.6. Finally, Sections 1.7 and 1.8 discuss thesis contributions and thesis structure, respectively. 


\subsection{Master-Slave Teleoperator Systems and Their Applications to Surgery}

According to Sheridan [1], teleoperation can be defined as the extension of a person's sensing and manipulation capabilities to a remote location. A standard teleoperator system consists of two (or more) robot manipulators called master and slave(s) connected through communication channels. The master manipulator is moved by a human operator, and the information about master's position and/or velocity (and, sometimes, forces applied by the human operator) is transferred over the communication channel to the remotely located slave. The slave is designed to follow the motion of the master, thus executing the remote task. In bilateral (also called bilaterally controlled or force-reflecting) teleoperator systems, the information about contact forces due to interaction with the environment (and, sometimes, position/velocity error) is sent back to the master site and applied to the motor of the master. Such a force feedback provides the human operator with a certain kinesthetic feeling of the interaction with the remote environment which may help to execute complex tasks better.

In master-slave surgical systems, a surgeon controls the operation from the master console, while the slave manipulator(s) follows the master command to perform a surgery on a patient. Generally, several areas can be identified where it may be advantageous to perform a surgery using a master-slave surgical robotic system. Below, three such areas are briefly considered: remote surgery, micro-surgery, and minimally-invasive surgery. 


\subsubsection{Remote Surgery}

Master-slave teleoperator system may be used to perform surgery in the situations where the surgeon(s) and the patient are spatially separated. One can imagine a number of situations where the presence of a surgeon on the patient's site is impossible or undesirable. Examples include the situations where a patient is in a geographically isolated area which is not accessible by a specialized surgeon within given time constraints; a surgery performed on a member of a space mission by a specialized surgeon located on Earth; a surgery performed on a patient in a combat zone, etc. Some of the most challenging problems that appear in this situation is based on the inevitable existence of communication delays between the surgeon and the patient sites, which results in surgical performance degradation and, in the case of force-reflecting teleoperators, delay-induced instability.

Telesurgery over very long distances is already proven to be feasible. In particular, the first trans-oceanic telerobotic surgery, entitled Operation Lindbergh, were performed in $2001[2 ; 3]$, when a group of surgeons located in New York City performed a laparoscopic cholecystectomy on a patient in Strasbourg, France. Although the operation was a success, several technical challenges were identified. In particular, formidable communication resources were committed in order to guarantee the quality of service (QoS) requirements. "Though we have demonstrated the concept and feasibility of remote telesurgery, we have done so using quite extensive (and expensive) telecommunications resources. It is realized that the demonstration as conducted in operation Lindbergh does not represent a cost-effective approach, nor does it constitute a viable scenario" [3]. In terms of communication resources, performing telesurgery over the Internet rather than over dedicated channels would greatly increase its accessibility and decrease cost. In particular, telesurgery systems that operate over the Internet would potentially allow global access to highly 
specialized surgeons.

\subsubsection{Micro-Surgery: Scaled Teleoperation}

Micro-surgery generally involves manipulation of tissue features of very small size, often of order of hundreds of microns. Human surgeons normally have very good dexterity at the natural human scale, however, their ability to operate deteriorates significantly outside the natural scale. In particular, humans have very limited abilities to perform precise motions at the very small geometric scale (particularly due to the human hands tremor) as well as to feel and control very small forces. Hence, significant research efforts were devoted to create telerobotic systems that can augment the human capabilities while performing surgery at the micro scale [4; $5 ; 6 ; 7]$. In microsurgical master-slave teleoperator systems, the positions (velocities) and forces are scaled appropriately between the master and the slave robots. For example, in microsurgical master-slave teleoperator system for eye surgery [5], the position of the master is scaled down 1 to 100 times before transmitting to the slave, while the forces experienced by the slave are scaled up 1 to 100 times before applying to the master. In particular, this allows surgeon to feel the interaction forces between the microsurgical tool and the eye which otherwise are too small to be felt. Such a microsurgical teleoperator system may also perform some accompanying tasks such as image augmentation and enhancement, as well as filtering out the hand tremor. Overall, development of practical master-slave teleoperator systems for microsurgery would allow surgeons to operate at unprecedented level of dexterity [4].

\subsubsection{Minimally-Invasive Surgery}

The notion of minimally-invasive surgery refers to a wide range of surgical procedures that can be performed without major incisions. Minimally-invasive surgical 
procedures are normally executed through small incisions using long handled specialized instruments, where endoscopic cameras provide the surgeon with a view of the internal operation field. The major benefit of this approach comparing to the traditional surgical procedures is that it is substantially less traumatic to the patient, which implies less pain, quicker recovery, decreased cost due to shorter hospital stay, and less cosmetic damage. Currently, however, the applicability of the minimallyinvasive surgical procedures is very limited due to insufficient development of the corresponding surgical equipment. Indeed, the current equipment frequently consists of one-degree-of-freedom tools that enter the human body through small incisions. Since the lateral movement is not possible at the incision, the instrument tip moves in the direction opposite to the one of the surgeon hand. Additionally, the surgeon normally watches the movement of the instrument on the display across the operation table, which makes the hand-eye coordination very difficult. Overall, the activities performed by the surgeon during minimally-invasive surgical procedures are very unnatural; as a result, every surgeon's motion must be deliberate rather than spontaneous as it is in traditional surgery [8]. Also, the surgeon is largely deprived of the sense of touch which normally plays a very important role. It is anticipated that the use of specialized master-slave force-reflecting teleoperator systems may remove the above mentioned obstacles and lead to effective minimally-invasive surgery.

\subsection{Importance of The Force Feedback}

In $[9 ; 10]$, the roles of the visual and the haptic (force) feedback in applications related to minimally invasive (laparoscopic) surgery were evaluated experimentally. In the experiments presented, tissue samples with different hardness characteristics were grasped with a specially designed laparoscopic grasper, and the subjects were 
asked to rank these samples from softest to hardest based on visual information, force feedback, or combination of the both. During experiments, subject opened and closed the jaws of the grasper using keyboard. The visual feedback was provided through video screen where subject was able to view the deformation of the samples. On the other hand, the force feedback were received through interaction with the PHANTOM device. The experiments clearly show that visual and force feedback used simultaneously result in better performance comparing to the cases where either visual feedback or force feedback were employed separately. (The other hypotheses of [9] was that the purely force feedback would provide better performance comparing to the purely visual feedback; it was not validated satisfactory).

In [11] (also [12]), the roles of the two components of the haptic feedback (namely, the force and the tactile components) in a suturing task were addressed. More precisely, in the experiments performed the suture forces applied during the first throw of the suture knot were measured and compared in the following three cases: i) hand ties, where complete force and tactile information is naturally provided; ii) ties performed by an instrument (needle driver) that provides complete force feedback but no tactile feedback, this case imitates a perfectly transparent force reflecting teleoperator system, and iii) ties performed by da Vinci ${ }^{T M}$ robot with no force feedback. The authors addressed the following two hypotheses. The first one states that the force magnitudes applied in case ii are indistinguishable from the case $i$, however, the force magnitudes in case iii are different. In other words, this hypothesis implies that the suture forces can be controlled more accurately with force feedback than without, however, the tactile feedback is not essential in this task. This hypothesis, however, was not validated by the experiments. In fact, it was found that, even though the forces in case ii are slightly closer to the case i comparing to case iii, there still is a significant difference between cases $i$ and 
ii. In other words, although the performance is improved in the presence of force feedback, the tactile feedback is probably crucial in maintaining higher accuracy. The second hypothesis was that the repeatability (described in terms of normalized standard deviation of the applied forces) in case ii is better comparing to case iii; this hypothesis was validated experimentally. The authors conclude that, since the current systems of robotic-assisted surgery continue to be limited in terms of tactile feedback, a sort of sensory substitution may be a short-term solution.

\subsection{Transparency and Fidelity}

One of the traditional goals of teleoperation is to achieve a complete transparency of the interface, i.e., to let the operator feel the task object as if it is being handled directly [13]. One possible definition of transparency can be given in terms of correspondence between position responses of the master $q_{m}$ and the slave $q_{s}$ manipulators as well as the correspondence between the forces exerted by the environment $f_{e}$ and the forces felt by the human operator $\hat{f}_{e}$ ("ideal response III" [14]): for any forces applied by the human operator $f_{h}(\cdot)$, the identities $q_{m}(\cdot) \equiv q_{s}(\cdot)$ and $f_{e}(\cdot) \equiv \hat{f}_{e}(\cdot)$ are to be satisfied regardless of the environmental dynamics in contact with the slave. A close (but somewhat more convenient for analysis especially using frequency domain methods) definition of transparency can be given in terms of impedance matching [15]: the impedance transmitted to or "felt" by the operator $Z_{t}$ is equal to the task or environmental impedance $Z_{e}$, i.e., $Z_{t}=Z_{e}$. In this case, the value of $\left\|Z_{t}-Z_{e}\right\|$ can serve as a measure of transparency.

In contrast with transparency, which is an unconditional requirement to feel the remote task "as is", fidelity is a task dependent definition of performance [16]. There are several reasons why, instead of optimizing for transparency, a teleoperator might 
be used to intentionally change the feeling of the environment. Such a change would be highly appropriate if, for example, master and slave operate on very different length, force, and/or power scales [17]. In this case, the goal is not (just) to enable remote manipulation, but rather to extend the capabilities of the human limbs. Examples are strength increasing devices like "man-amplifiers" or "extenders" [18] on one hand, and dexterity increasing macro-micro bilateral teleoperation on the other. The latters are enjoying increasing applications to microsurgery, in particular, microsurgical augmentation (see [19] and bibliography therein).

Other fidelity measures are also possible. For example, as it is pointed out in [20], it may be desirable to design a system that helps a surgeon to improve the ability to detect the changes in environmental compliance. This observation motivates the choice of fidelity measure in the form of a (weighted) sensitivity of the transmitted impedance $Z_{t}$ to changes in the environmental impedance $Z_{e}$, i.e.,

$$
\left\|\left.W_{s} \frac{d Z_{t}}{d Z_{e}}\right|_{Z_{e}=\hat{Z}_{e}}\right\|_{2}=\sqrt{\left.\frac{1}{2 \pi} \int_{-\infty}^{+\infty}\left|W_{s}(j \omega) \frac{d Z_{t}(j \omega)}{d Z_{e}(j \omega)}\right|_{Z_{e}(j \omega)=\hat{Z}_{e}(j \omega)}\right|^{2} d \omega},
$$

where $\hat{Z}_{e}$ is a nominal environmental impedance and $W_{s}$ is a weighting function. The design goal addressed in [20] is to maximize the minimum of the norm (1.1) over a given set of nominal environment impedances $\hat{\mathbb{Z}}_{e}$ subject to robust stability and tracking constraints.

\subsection{Stability vs. Transparency Trade-Off}

Stability is a requirement of paramount importance for majority of engineering systems, including teleoperators. One can think of an "ideal" teleoperator system as the one which is perfectly transparent and perfectly stable at the same time. The relation between stability and transparency is one of the central topics in the literature 
on the teleoperator system design. In particular, Lawrence [15] studied relation between stability (more precisely, passivity) and transparency for several teleoperator schemes, including position-position, position-force, passivated position-force [21], as well as "transparency optimized" teleoperator systems architecture proposed in [15]. In all these cases, it is found that passivity and transparency are conflicting goals; more precisely, using an appropriate choice of design parameters, a teleoperator system can be made more passive (stable) at the expense of decreased transparency, and vice versa. This fact can be illustrated by the following simple example [21]. Consider a master-slave teleoperator system described by two-port Hannaford's model of the form

$$
\left[\begin{array}{c}
T_{m} \\
-V_{s}
\end{array}\right]=\left[\begin{array}{ll}
h_{11} & h_{12} \\
h_{21} & h_{22}
\end{array}\right]\left[\begin{array}{l}
V_{m} \\
T_{s}
\end{array}\right]
$$

Suppose the master-slave system is designed to be perfectly transparent. This implies that the parameters of the hybrid matrix are $h_{11}=h_{22}=0, h_{12}=1$, and $h_{21}=-1$. It can also be obtained by direct verification that the system is passive and therefore stable. However, it can also be shown that this system is marginally stable, and an arbitrary small disturbance may destabilize it. For example, existence of even a small delay in the communication channel destroys the stability properties of such a system. Indeed, let $T>0$ be an arbitrarily small time delay. In presence of communication delay $T>0$ the system becomes

$$
\left[\begin{array}{c}
T_{m} \\
-V_{s}
\end{array}\right]=\left[\begin{array}{cc}
0 & e^{-s T} \\
-e^{-s T} & 0
\end{array}\right]\left[\begin{array}{c}
V_{m} \\
T_{s}
\end{array}\right] .
$$

This system is not passive. It can be shown by calculation a norm of a scattering operator. Scattering operator of a system can be found based on its hybrid matrix 
$H(s)$ as follows

$$
S(s):=\left[\begin{array}{cc}
1 & 0 \\
0 & -1
\end{array}\right](H(s)-\mathbb{I})(H(s)+\mathbb{I})^{-1} .
$$

It is well-known fact that the system is passive if and only if $\|S(s)\| \leq 1$. Calculating the scattering operator for the system (1.2) gives

$$
\begin{gathered}
S(s)=\left[\begin{array}{cc}
1 & 0 \\
0 & -1
\end{array}\right]\left[\begin{array}{cc}
-1 & e^{-s T} \\
-e^{-s T} & -1
\end{array}\right]\left[\begin{array}{cc}
1 & e^{-s T} \\
-e^{-s T} & 1
\end{array}\right]^{-1} \\
=\left[\begin{array}{cc}
-\tanh (s T) & \cosh ^{-1}(s T) \\
\cosh ^{-1}(s T) & \tanh (s T)
\end{array}\right],
\end{gathered}
$$

and its norm

$$
\|S(s)\|=\sup _{\omega}(|\tan (\omega T)|+|\sec (\omega T)|)=\infty .
$$

Thus, $S(s)$ is an unbounded operator, therefore (1.2) is not passive, and the stability is not guaranteed.

For the force-reflecting teleoperator systems, the stability vs. transparency tradeoff can also be explained in terms of the closed-loop gain. Namely, for transparent teleoperation, the contact force due to environment must be sent to the master with high enough force-reflecting gain; however, this would increase closed-loop gain and therefore decrease stability margin. In this thesis, the contradiction between stability and high force reflecting gain is addressed, and a new solution is proposed (Chapter 4).

\subsection{The Effect of Communication Delay}

Communication delays are intrinsic for teleoperation over long distances. By obvious physical reasons, communication delays can never be avoided despite of any possible 
technological development. Existence of a delay in the communication channel of a telesurgical system imposes several limitations and presents certain technological challenges. Here, we consider three aspects of communication delay impact on telesurgical performance. The first one is how the delay affects the ability of a surgeon to perform operations. The second and third aspects are how the existence of communication delay affects transparency and stability characteristics of a highperformance teleoperator system, respectively.

\subsubsection{The Effect of Time-Delay on Performance of Telesurgery}

Generally, the existence of time delays in the communication channels of a teleoperator system results in a loss of the sense of causality for the human operator. An important question is what is the maximal amount of communication delay that does not compromise significantly the ability of a surgeon to operate. This question has been addressed in $[22 ; 3]$. In particular, the results of [22] suggest that a laparoscopic surgeon can operate successfully in the presence of communication delay up to $700 \mathrm{~ms}$. In [3], similar study has been performed as a part of preparation to the first transatlantic laparoscopic cholecystectomy (2001). Surgical experiments were performed over a teleoperator system which was subject to different communication latency settings. It was generally agreed by participants that the "comfort zone" to perform telesurgery was up to $330 \mathrm{~ms}$ of round-trip delay, although some surgeons felt that they can adapt to delays up to $500 \mathrm{~ms}$ [3]. 


\subsubsection{Transparency in The Presence of Time Delay}

The existence of time delay in the communication channel imposes fundamental limitations on the performance of the teleoperator system. Indeed, after the human operator moves the master manipulator, the corresponding reaction of the environment cannot be felt earlier than the round trip delay time $T$. This implies that, in the presence of communication delay, it is technically impossible to achieve transparency in the sense defined by Yokokohjii and Yoshikawa ("Ideal Response III" [14]) or by Lawrence (impedance matching [15]). Although the complete transparency is not achievable, it is still possible to guarantee certain relaxed versions of transparency, such as delayed kinematic correspondence [23]. Another important concept related to transparency in presence of time delay is the concept of Virtual Tool introduced by Niemeyer [24]. The idea behind this concept is that, in the presence of communication delay, it makes sense to pursue a goal of creating a simple and predictable dynamics of the teleoperator system rather than trying to completely eliminate them (the latter corresponds to the case of perfect transparency).

\subsubsection{Stability in The Presence of Time Delay}

Existence of time delay in the communication channel may create stability problems in force-reflecting teleoperator systems. This fact was first established in early experiments performed by Ferrell in 1966 [25]. In this work, it is shown that instability may arise even in the presence of time delay as small as $0.1 \mathrm{sec}$. Theoretically, the time delay in the communication channel creates an unpredictable phase shift of the closed-loop system which may destroy stability of passivity-based schemes. Since then, significant research effort have been devoted to overcome delay-induced instability. The first solution to this problem had been proposed by Anderson and Spong in 1989 [21]. Their solution was based on so-called Caley feedback trans- 
formation. This idea was further developed by Niemeyer and Slotine [26], where the approach of Anderson and Spong was extended to the case of position-position teleoperator architecture, and the feedback Caley transformation was interpreted in terms of wave variables. Since then, a number of solutions have been proposed based on different ideas. Among these, there are several works that further developed the application of wave-variables concept $[27 ; 28 ; 24]$. In the shared compliant control approach [29], a special local force feedback is implemented on the slave side which results in improved stability properties. The work of Leung, Francis, and Apkarian [30] applies $\mathcal{H}_{\infty}$ optimal control and $\mu$-analysis and synthesis techniques to design a system which is robust with prescribed time-delay stability margin. In [31], independent adaptive motion/force controllers are implemented on both the master and the slave side to guarantee stable teleoperation in presence of time delay. These schemes and some others are analyzed from unified point of view in survey paper [32].

All the above mentioned schemes, however, have some common limitations. First, stability of these schemes are achieved at the expense of transparency deterioration. Second, stability of all of these schemes is guaranteed only in the case of constant communication delay; in fact, stability in presence of time-varying significantly irregular communication delay is not addressed in the above mentioned works. The case of time-varying irregular communication delay, however, appears to be of significant practical importance, since this is a common situation when dealing with teleoperation over the Internet. It is a well-known fact [33] that the stability in the presence of arbitrary constant communication delay does not generally imply the stability in the presence of time varying-delay; therefore, time-varying case requires separate proof. Recently, a number of works are published which propose stabilization schemes for bilateral teleoperation with time-varying communication 
delay. In particular, partial extensions of wave-variable approach to the case of time-varying communication delay were presented in $[34 ; 35]$. A wave variable with prediction approach is applied to teleoperation over the Internet in [27]. Sliding mode control approach in combination with impedance control on the master side is presented in [36]. In [37], a control scheme is presented which stabilize teleoperator in presence of slowly-varying communication delay, while the fast varying component is filtered using a Kalman filter. In the paper [38], which describes a real-life industrial project of force-reflecting teleoperation over the Internet, a combination of buffering mechanism and a predictor is utilized to cope with time-varying delay.

Overall, there is a large body of work done in developing stabilization schemes for bilateral teleoperation with time delay, including time-varying case. However, the proof of stability of the above described schemes still requires imposing significant constraints on communication process, which may be hard to justify in the case of

the Internet-based teleoperation. In other words, there is lack of general stability results for teleoperator system with significantly irregular communication delays. This is one of the central problem addressed in this thesis.

\subsection{Design Guidelines for Telesurgery Systems}

Although different telesurgery systems may have their specifics, a set of general design guidelines can be identified. Ideally, the slave device should track the master trajectory as precisely as possible, and the surgeon should feel no forces/torques other than the interaction between the tool and the tissue on the patient side. These general considerations can be specified using the following list of design requirements.

1. Low damping of the master device. Again, high damping of the master device would require the surgeon to apply excessive forces in order to execute the 
operations and lead to transparency deterioration, since the local damping forces would interfere with the forces reflected from the surgical site.

2. High stiffness of the overall master-slave interconnection. In particular, this includes the requirement that the slave must precisely track the trajectory of the master, and demonstrate high impedance against the environmental force while tracking the master trajectory.

3. High bandwidth and high enough force reflection gain. Both these are necessary to provide the surgeon with a crisp feeling of the contact against obstacles on the patient side.

4. In presence of significant communication delay, it may be desirable to incorporate some force regulation on the slave side.

Note that the combination of requirements 1-3 is in natural contradiction with the stability of the overall teleoperator system in the presence of time delays.

\subsection{Thesis Contributions}

The thesis deals with different issues that arise in master-slave teleoperator systems that operate over wide-area networks. More precisely, we present a set of results that aims at achieving stable and transparent force-reflecting teleoperation in the presence of network induced communication constraints that include but are not limited to irregularity of transmission intervals, existence of time-varying possibly unbounded communication delays, and possible packet losses. Specifically, the key contributions of this thesis can be identified as follows.

- In this thesis, the IOS/ISS small gain approach to bilateral teleoperation in the presence of network induced communication constraints is systematically 
developed. The approach is based on a new version of the IOS/ISS small gain theorem that is applicable to systems where communication between subsystems is performed over multiple channel and is subject to significant communication constraints such as time-varying discontinuous possibly unbounded communication delays and possible information losses. Based on this approach, schemes for bilateral teleoperation are designed that guarantee stability in the presence of irregular communications. Additionally, tracking properties are guaranteed in the case of sufficiently smooth communication delay in the forward channel.

- The projection-based force reflection algorithms are introduced and systematically developed. The idea behind these algorithms is to attenuate the component of the external forces that is not compensated by the human hand; such an attenuation does not affect the human perception of the force, however, it removes the component of the force reflection that can potentially destroy the system's stability. In particular, the proposed approach allows to achieve the stability for an arbitrarily low damping of the master manipulator and arbitrarily high force reflection gain; thus, it solves the trade-off between stability, maneuvrability, and high force reflection gain in bilateral teleoperation with time-varying irregular communication delay.

- A model-based approach to the design of bilateral teleoperators enhanced by means of virtual environment in the presence of communication constraints is presented. This approach develops ideas from sampled-data nonlinear systems design based on partial knowledge of a set of approximate discrete-time models. The virtual environment block runs the remote state estimation, identification, and trajectory prediction algorithms that are based on an available model of 
the remote process and delayed possibly corrupted measurements. An essential and distinct feature of the approach presented is the simultaneous design of a control algorithm and a communication protocol that together guarantee the overall scheme stability and performance in the presence of significant network induced communication constraints.

\subsection{Thesis Structure}

The thesis has the following structure. In Chapter 2, a new multi-channel version of the IOS small gain theorem for systems with multiple time-varying communication delays is presented. This theorem is derived specifically for the stability analysis of the interconnections where the communication between subsystems is performed over multiple channels, and is subject to unknown, time-varying and possibly unbounded communication delays; moreover, delays in different channels may have independent characteristics. For this type of interconnections, a "multi-dimensional" small gain stability condition is established. This theorem is our main technical tool that allows investigating the stability of different teleoperation schemes presented in the subsequent chapters.

In Chapter 3, schemes for stable bilateral teleoperation are presented that are based directly on the proposed version of the IOS small gain theorem. The schemes proposed essentially utilize local control algorithms that make the master and the slave subsystems ISS/IOS; the slave algorithm is also designed to make the slave track the (delayed) trajectory of the master. We address a general form of force reflection term as a (possibly trivial) linear combination of the slave position error, slave velocity error and the actual contact force/torque due to environment, and prove that the teleoperator scheme proposed guarantees stable contact with the en- 
vironment under mild assumptions on communication delays. Tracking properties are also proven under an additional assumption that the communication process in the forward communication channel is approximately smooth in some sense. Theoretical results presented in this chapter are confirmed by experiments.

In Chapter 4, the trade-off between closed-loop teleoperator stability and high force reflecting gain is addressed. To solve this trade-off, projection-based force reflection algorithms are introduced. These algorithms use the fact that the human perception of an external force depends critically on the direction and the magnitude of her/his own forces applied to the master manipulator. The idea behind the projection-based force reflection (FR) algorithms, therefore, is to alternate the force reflection signal depending on the forces applied by the human operator; it can be done in such a way that the human does not feel this alteration, however, the resulting induced master motion can be eliminated or reduced to an appropriate level. The approach presented allows to achieve an arbitrarily high force reflection gain without loosing the overall stability and/or without increasing the damping and the stiffness of the master; thus, it solves the contradiction between stability and high force reflection gain in bilateral teleoperation with time-varying irregular communication delay. The theoretical results presented in this section are also confirmed by experiments.

In Chapter 5, an alternative approach to teleoperation in the presence of delayed force feedback is presented. In this approach, a virtual environment is created on the master side that duplicates the essential features of the remote slave+environment interconnection. In this case, the human operator may use the local force feedback generated by the virtual "slave+environment" model in order to obtain certain "feeling" the process. Such a local force feedback is not subject to communication delay, which restores the sense of causality for the human operator and does not have the 
destabilization effect of the delayed force feedback. The approach presented is based on further development of some ideas from model-based sampled data stabilization of nonlinear systems based on a set of approximate models. In particular, we present a control algorithm and a communication protocol that deals with teleoperation in presence of time-varying unknown communication delays and possible packet losses. The theoretical results presented in this section are confirmed by simulations.

In Chapter 6, the contribution of the thesis is briefly discussed, and future research directions are identified.

Finally, Appendix A presents a complete derivation of the Phantom ${ }^{T M}$ kinematic and dynamic models, while communication aspects of telesurgery over the Internet are briefly discussed in Appendix B. 


\section{Chapter 2}

\section{Small-Gain Approach to}

\section{Network-Based Teleoperation}

The goal of this Chapter is to present, together with all the necessary preliminaries, a new version of the IOS small-gain theorem designed specifically for the systems that communicate over multiple networked channels. This theorem serves as the main tool for stability analysis of the force-reflecting teleoperator schemes presented subsequently in Chapters 3 and 4 . The structure of the Chapter is as follows. Introductory discussion together with some historical remarks on small-gain theorems are given in Section 2.1. In Section 2.2, some specific features of the network-based teleoperators are discussed that motivate the particular form of the small gain result presented in this work. Section 2.3 introduces main definitions and contains preliminary technical material. Section 2.4 presents a mathematical description of the network-based interconnections, in particular, constraints imposed on communication process are discussed. The IOS small gain theorem together with its proof are given in Section 2.5. An illustrative example is presented in Section 2.6, while concluding remarks are given in Section 2.7 . 


\subsection{Introduction: Stability and Small Gain The-}

\section{orems}

The small-gain theorem is possibly one of the most important results in feedback systems theory. In its simplest form, it asserts that a feedback system is stable if the product of the subsystem's gains is less than one. After its establishment in 1960s $[39 ; 40]$, the small gain theorem has found numerous applications in many areas of systems and control design. Some important references on this topic include $[40 ; 41 ; 42 ; 43 ; 44]$, among many others (see also a recent survey [45] devoted to nonlinear control applications). In particular, extensions of the small gain theorem to the case of input-to-state stable (ISS, [46]) subsystems have been developed $[43 ; 44]$. In modern control applications, communication between subsystems is frequently performed over communication networks, such as local area networks (LANs) as well as the Internet; network-based teleoperation is a good example of such an application. In particular, in advanced teleoperator systems, the master and the slave manipulators may exchange information of different nature such as positions, velocities, forces, and video. These signals come from different sensors and may be sent asynchronously over multiple network connections which results in different delay/packet losses characteristics.

In this Chapter, we present a version of the IOS Small-Gain Theorem that is designed specifically for the stability analysis of the interconnections where the communication between subsystems is performed over multiple channels, and is subject to time-varying possibly unbounded communication delays. Comparing to the wellestablished versions of the IOS (ISS) small gain theorem [43; 44], our work differs in at least two aspects. First, we consider multi-input-nulti-output (MIMO) subsystems where a separate gain function is associated with each input-output channels 
pair, and address stability properties of the interconnection which is carried out over multiple channels in both the feedforward and the feedback paths. For this type of interconnections, a "multi-dimensional" small gain stability condition is established. This approach results in significantly less conservative stability criteria comparing to the traditional one, where the maximal gain over all channels are taken into consideration. In particular, in many multi-channel systems such as teleoperators, some gains but usually not all of them can be assigned arbitrarily, therefore, using the small-gain theorem based on the only one gain function that works for all the channels may lead to a wrong conclusion about impossibility to stabilize the system. Second, we formulate and prove the IOS small gain theorem for the case where the communications between subsystems is subject to unknown, time-varying and possibly unbounded communication delays, and moreover, delays in different channels may have independent characteristics. To deal with stability analysis of systems with multiple time-varying delays, we use a (multi-channel) extension of the IOS notion to systems of functional differential equations (FDEs) analogous to the one of the input-to-state stability (ISS) proposed in [47]. We prove that if both subsystems are IOS then a "multi-channel" version of the strict contraction ("small gain") condition implies that the interconnected system, being considered as a system of FDEs, is IOS if the communication delays satisfy certain mild assumptions. We show that the fulfillment of these assumptions does not depend on characteristics of the communication channels; on the contrary, it can always be guaranteed by implementation of certain standard features of the underlying communication protocol such as packet numbering and (or) timestamping.

References relevant to the result presented in this Chapter include [48; 49]. In particular, in [48] the ISS small gain arguments are applied to the problem of stabilization of nonlinear systems in presence of quantization and bounded communi- 
cation delays, while in [49] small-gain theorems for monotone dynamical systems are established that are suitable for treatment of delay-differential equations with multiple inputs and outputs. The results presented in this Chapter form a basis for the stability analysis of the force-reflecting teleoperation schemes presented in the subsequent parts of this thesis.

\subsection{Features of the Network-Based Teleoperators}

\subsubsection{Sampled-Data Implementation}

In the network-based teleoperator systems, the master and slave subsystems normally consist of a mechanical device interconnected with a digital controller. Thus, the subsystems can naturally be described as sampled-data systems (possibly with quantization). Analytically, a sampled-data nonlinear system can be described either as a continuous-time system with time-varying delays (as proposed for example in $[50 ; 51])$, or represented by its discrete-time model [52]. Although both these approaches have their merits and advantages, the continuous-time modelling approach seems to be preferable over the discrete-time one in at least two aspects. First, modelling of a continuous-time nonlinear plant in the discrete-time domain requires exact integration of the corresponding differential equations, which is generally not possible for a nonlinear system. Such a lack of exact integration in the nonlinear case was the main reason for development of the methods for sampled-data stabilization of nonlinear systems based on a set of approximate discrete-time models [52; 53]. Second, the continuous-time approach appears to be more complete, because the continuous-time plant always exhibits some inter-sampling dynamics which are ignored in the discrete-time approach. In particular, stability of a discrete-time model does not necessarily imply stability of the corresponding sampled-data system, since 
the intersampling behaviour of a continuous-time plant may exhibit peaking (see, for example [54]). Because of these two reasons, we choose to use a continuoustime framework, where the components of the interconnection are described by functional-differential equations (that, in particular, include retarded differential equations with time-varying delays as a special case).

\subsubsection{Multiple Channels}

In advanced master-slave teleoperator systems, the subsystems may exchange information of different nature, such as positions/velocities of the manipulators, contact forces/torques, visual information, as well as some auxiliary signals. These signals may be sent over multiple network connnections with different QoS characteristics. Also, these signals may have different influence on the processes in the other subsystem; for example, in terms of small gain design, the subsystems may have different gains with respect to different input signals. It can be shown that modelling of the teleoperator system as an interconnection with multiple communication channels may lead to significantly less conservative design comparing to the case where only one channel in each direction is taken into account. This fact is illustrated by an example presented below in Section 2,6.

\subsubsection{Communication Constraints}

Having a network such as the Internet as a communication medium implies existence of certain communication constraints. For example, packets in the Internet may follow different routes, and the transmission delay along each route depends significantly on current state of that route, existence of congestions, routers queue lengths, etc. As a result, transmission delay of each particular packet is unknown beforehand and may differ significantly from packet to packet. Also, occurrence 
of congestions may result in unbounded communication delays and packet drops. All these phenomena complicate the analysis and control of systems that include networks as a communication medium, and this fact recently stimulated significant research efforts in the area of networked control systems (see [55], and bibliography therein).

\subsection{Technical Preliminaries}

\subsubsection{Basic Notation and Definitions}

Throughout this work, the notion of functional classes $\mathcal{N}, \mathcal{G}, \mathcal{K}, \mathcal{N}_{\infty}, \mathcal{K}_{\infty}$, and $\mathcal{K} L$ will be used extensively. These classes are defined as follows (see, for example, [56]). By $\mathbb{R}_{+}$we denote the set of nonnegative real numbers, $\mathbb{R}_{+}:=[0,+\infty)$. A continuous function $\gamma: \mathbb{R}_{+} \rightarrow \mathbb{R}_{+}$is said to belong to class $\mathcal{N}(\gamma \in \mathcal{N})$ if it is nondecreasing. A function $\gamma \in \mathcal{N}$ is said to belong to class $\mathcal{G}(\gamma \in \mathcal{G})$ if it satisfies $\gamma(0)=0$. A function $\gamma \in \mathcal{G}$ belongs to class $\mathcal{K}(\gamma \in \mathcal{K})$ if it is strictly increasing. Furthermore, the classes $\mathcal{N}_{\infty}$ and $\mathcal{K}_{\infty}$ are defined as follows: a function $\gamma \in \mathcal{K}(\gamma \in \mathcal{N})$ belongs to class $\mathcal{K}_{\infty}\left(\mathcal{N}_{\infty}\right)$ if $\gamma(s) \rightarrow \infty$ as $s \rightarrow \infty$. Finally, $\beta: \mathbb{R}_{+} \times \mathbb{R}_{+} \rightarrow \mathbb{R}_{+}$is said to belong to class $\mathcal{K} L(\beta \in \mathcal{K} L)$, if $\beta(\cdot, b)$ is a $\mathcal{K}_{\infty}$-class function for each fixed $b \geq 0$, and $\beta(a, \cdot)$ is strictly decreasing for each fixed $a>0$.

\subsubsection{Multidimensional Extensions of $\mathcal{G}, \mathcal{K}, \mathcal{K}_{\infty}$-Classes}

Below, we will frequently deal with stability properties of multiple inputs - multiple outputs (MIMO) systems. To simplify the notation in MIMO case, it is desirable to use an extension of the classes $\mathcal{G}, \mathcal{K}$, and $\mathcal{K}_{\infty}$ to the case of multivariable maps $\mathbb{R}_{+}^{m} \rightarrow \mathbb{R}_{+}^{n}$, where $\mathbb{R}_{+}^{n}$ is a set of n-tuples of nonnegative real numbers, and $m, n \in \mathbb{N}$. One possible way to define such an extension is to consider $n \times m$-dimensional arrays 
of functions $\Gamma_{i j}: \mathbb{R}_{+} \rightarrow \mathbb{R}_{+}, i=1,2, \ldots, n, j=1, \ldots, m$. With each such an array, a map $\Gamma: \mathbb{R}_{+}^{m} \rightarrow \mathbb{R}_{+}^{n}$ can be associated which is defined for each $\mathbf{s}=\left(s_{1}, s_{2}, \ldots, s_{m}\right)^{T} \in$ $\mathbb{R}_{+}^{m}$ according to the formula

$$
(\boldsymbol{\Gamma}(\mathbf{s}))_{i}=\max _{j \in\{1, \ldots, m\}} \Gamma_{i j}\left(s_{j}\right), \quad i=1, \ldots, n .
$$

The map $\Gamma: \mathbb{R}_{+}^{m} \rightarrow \mathbb{R}_{+}^{n}$ is said to belong to a class $\mathcal{G}^{n \times m}$ if all $\Gamma_{i j}$ belong to class $\mathcal{G}$. Classes $\mathcal{K}^{n \times m}$ and $\mathcal{K}_{\infty}^{n \times m}$ are defined analogously.

Some further notations are as follows. Similarly to the scalar case, the composition of maps $\Gamma_{1} \in \mathcal{G}^{n \times m}$ and $\Gamma_{2} \in \mathcal{G}^{m \times p}$ is denoted by $\Gamma_{2} \circ \Gamma_{1} \in \mathcal{G}^{n \times p}$, i.e., $\Gamma_{2} \circ \Gamma_{1}(s):=\Gamma_{2}\left(\Gamma_{1}(s)\right)$. For vectors in $\mathbb{R}_{+}^{l}$, we use relations $\leq,<, \geq,>$ defined as follows: given $\mathbf{u}_{1}, \mathbf{u}_{2} \in \mathbb{R}_{+}^{l}$, we will write $\mathbf{u}_{1} \leq \mathbf{u}_{2}$ if and only if $u_{1}^{\{i\}} \leq u_{2}^{\{i\}}$ for all $i \in\{1, \ldots, l\}$, and relations $<, \geq,>$ are defined analogously. Finally, the maximum of $\mathbf{u}_{1}, \mathbf{u}_{2} \in \mathbb{R}_{+}^{l}$ should be understood in the elementwise sense, 1.e., $\mathbf{u}:=\max \left\{\mathbf{u}_{1}, \mathbf{u}_{2}\right\} \in\left(\mathbb{R}^{+}\right)^{l}$ consists of elements $u^{\{i\}}:=\max \left\{u_{1}^{\{i\}}, u_{2}^{\{i\}}\right\}, i=1, \ldots, l$.

\subsubsection{Input-to-State Stability and Input-to-Output Stabil- ity of Functional-Differential Equations}

The notion of the input-to-output stability (IOS) as well as the related notion of the input-to-state stability (ISS) were first introduced for the case of time-invariant ordinary differential equations (ODEs) in [57]. Since then, these notions were thoroughly developed (see for example [46] and the extensive bibliography therein). In particular, paper [47] deals with the notion of ISS for systems of functional-differential equations (FDEs). This paper is of a special interest for our work, since we address stability properties of the interconnections where the subsystems communicate over network channels that may involve communication delays. To describe such an interconnection, a system of FDEs would be an appropriate mathematical object. 
Below, we use the notation borrowed from [58; 47]. Given functions $x: \mathbb{R} \rightarrow \mathbb{R}^{n}$, $t_{d}: \mathbb{R} \rightarrow \mathbb{R}_{+}$, denote $x_{d}(t):=\left\{x(s), s \in\left[t-t_{d}(t), t\right]\right\}$. Thus, $x_{d}(t)$ is a piece of trajectory which begins at $s=t-t_{d}(t)$ and ends at $s=t$, rather than simply its value at time $t$. Now, given a function $t_{d}: \mathbb{R} \rightarrow \mathbb{R}_{+}$satisfying $t_{d}\left(t_{2}\right)-t_{d}\left(t_{1}\right) \leq t_{2}-t_{1}$ for all $t_{1}, t_{2} \in \mathbb{R}$, consider a system of functional differential equations where both the input and the output are partitioned into separate "channels", as follows

$$
\begin{aligned}
\dot{x}(t) & =F\left(x_{d}, u_{d}^{\{1\}}, \ldots, u_{d}^{\{l\}}, t\right), \\
y^{\{1\}}(t) & =H^{\{1\}}\left(x_{d}, u_{d}^{\{1\}}, \ldots, u_{d}^{\{l\}}, t\right), \\
\vdots & \\
y^{\{r\}}(t) & =H^{\{r\}}\left(x_{d}, u_{d}^{\{1\}}, \ldots, u_{d}^{\{l\}}, t\right) .
\end{aligned}
$$

Here, $u^{\{i\}} \in \mathbb{R}^{m_{i}}, i \in\{1, \ldots, l\}$ are the inputs, and $y^{\{j\}} \in \mathbb{R}^{p_{j}}, j \in\{1, \ldots, r\}$ are the outputs, $\Sigma_{i=1}^{l} m_{i}=m, \Sigma_{j=1}^{r} p_{j}=p$. Note that in the special case where $t_{d}(\cdot) \equiv 0,(2.1)$ becomes a system of ODEs. It is assumed that $F$ and $H^{\{j\}}, j=$ $\{1, \ldots, r\}$ are Lipschitz continuous operators in $x_{d}, u_{d}$ uniformly for all $t \in \mathbb{R}$, and Lebesgue measurable in $t$. Traditional definition of the IOS deals with system with one input and one output, so that the stability is characterized by a single IOS gain function (although benefits of using different gain functions for different inputs were mentioned, for example, in [43]). For a MIMO system of the form (2.1), however, it may be desirable to use a definition of IOS (ISS) where separate gain functions are specified for each pair of the input-output channels. We present such a definition using the multidimensional extension of the classes $\mathcal{G}, \mathcal{K}$, and $\mathcal{K}_{\infty}$ introduced above. Below, we use the notation $\left|x_{d}(t)\right|:=\sup _{s \in\left[t-t_{d}(t), t\right]}|x(s)|$ which is adopted from [47], and we denote $\mathbf{u}_{d}^{+}:=\left[\left|u_{d}^{\{1\}}\right|, \ldots,\left|u_{d}^{\{l\}}\right|\right]^{T} \in \mathbb{R}_{+}^{l}, \mathbf{y}^{+}:=\left[\left|y^{\{1\}}\right|, \ldots,\left|y^{\{r\}}\right|\right]^{T} \in \mathbb{R}_{+}^{r}$, where $|\cdot|$ denotes the vector $\infty$-norm. A multi-channel version of the IOS notion for systems of FDEs of the form (2.1) can be defined as follows. 
Definition 1. (IOS/ISS for FDEs) The system of the form (2.1) is input-tooutput stable (IOS) at $t=t_{0} \in \mathbb{R}$ with $t_{d}\left(t_{0}\right) \geq 0$, IOS gains $\Gamma \in \mathcal{G}^{r \times l}$, restrictions $\Delta_{x} \in \mathbb{R}_{+}, \Delta_{u} \in \mathbb{R}_{+}^{l}$, and offset $\delta \in \mathbb{R}_{+}^{r}$ if the conditions $\left|x_{d}\left(t_{0}\right)\right| \leq \Delta_{x}$, and $\sup _{t \geq t_{0}} \mathbf{u}_{d}^{+} \leq$ $\Delta_{u}$, imply that the solution of (2.1) are well-defined for all $t \geq t_{0}$, and the following properties hold:

i) uniform boundedness: there exists a function $\beta \in \mathcal{K}_{\infty}^{r \times 1}$ such that

$$
\sup _{t \geq t_{0}} \mathbf{y}^{+}(t) \leq \max \left\{\beta\left(\left|x_{d}\left(t_{0}\right)\right|\right), \Gamma\left(\sup _{t \geq t_{0}} \mathbf{u}_{d}^{+}(t)\right), \delta\right\}
$$

ii) convergence:

$$
\limsup _{t \rightarrow \infty} \mathbf{y}^{+}(t) \leq \max \left\{\boldsymbol{\Gamma}\left(\limsup _{t \rightarrow \infty} \mathbf{u}_{d}^{+}(t)\right), \delta\right\} .
$$

The system (2.1) is said to be input-to-state stable (ISS), if the above conditions hold for $\mathbf{y}^{+}:=|x|$.

Remark 1. As mentioned above, in the special case where $t_{d}(\cdot) \equiv 0$, system (2.1) becomes a system of ODEs. Similarly, the above defined multi-channel notion of IOS/ISS can be specialized to the case of systems of ODEs by imposing the same restriction, i.e., $t_{d}(\cdot) \equiv 0$. Throughout this work, we will use the above notions for systems of FDEs as well as systems of ODEs; in the latter case, $t_{d}(\cdot) \equiv 0$ is assumed.

\subsection{Network-Based Interconnections}

Consider two systems of FDEs $\Sigma_{i}, i \in\{1,2\}$, of the form

$$
\begin{aligned}
\dot{x}_{i} & =F_{i}\left(x_{i d}, u_{i d}^{\{1\}}, \ldots, u_{i d}^{\left\{l_{i}\right\}}, w_{i d}^{\{1\}}, \ldots, w_{i d}^{\left\{v_{i}\right\}}, t\right), \\
y_{i}^{\{1\}} & =H_{i}^{\{1\}}\left(x_{i d}, u_{i d}^{\{1\}}, \ldots, u_{i d}^{\left\{l_{i}\right\}}, w_{i d}^{\{1\}}, \ldots, w_{i d}^{\left\{v_{i}\right\}}, t\right), \\
\vdots & \\
y_{i}^{\left\{r_{i}\right\}} & =H_{i}^{\{r\}}\left(x_{i d}, u_{i d}^{\{1\}}, \ldots, u_{i d}^{\left\{l_{i d}\right\}}, w_{i d}^{\{1\}}, \ldots, w_{i d}^{\left\{v_{i}\right\}}, t\right),
\end{aligned}
$$




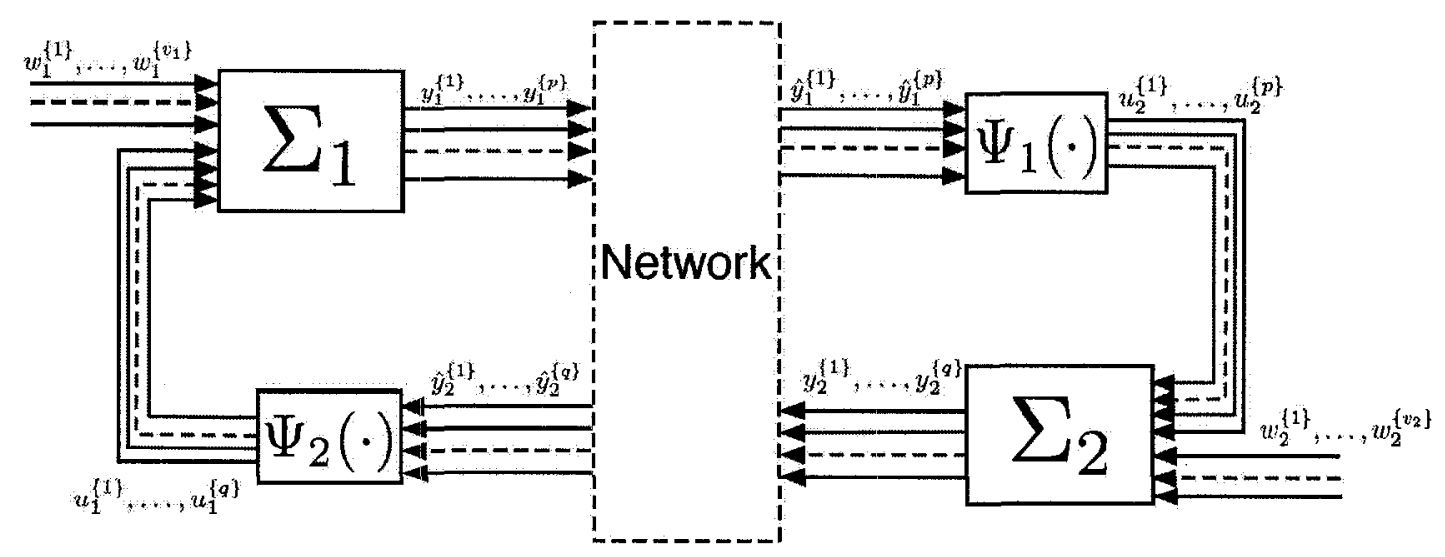

Figure 2.1: Network-based interconnection

where the inputs are categorized into the controlled inputs $u$ and disturbances inputs $w, r_{1}=l_{2}=p \in \mathbb{N}, r_{2}=l_{1}=q \in \mathbb{N}, v_{1}, v_{2} \in \mathbb{N}$. As before, both $t_{i d}(t), i \in\{1,2\}$, are assumed to satisfy $t_{i d}\left(t_{2}\right)-t_{i d}\left(t_{1}\right) \leq t_{2}-t_{1}$ for all $t_{1}, t_{2} \in \mathbb{R}$.

Remark 2. In the special case where $t_{1 d}(t) \equiv 0$ and/or $t_{2 d}(t) \equiv 0$, the corresponding subsystem(s) becomes a system of ODEs. Our choice to describe subsystems $\Sigma_{i}, i \in\{1,2\}$ in terms of FDEs is based on the fact that, in many cases, the subsystems that communicate over a network are implemented in sampled-data fashion. Such a sampled-data system can naturally be represented as a system of FDEs with uniformly bounded $t_{d}(\cdot)$ (more precisely, as a system of retarded differential equations with time-varying uniformly bounded delays) [50].

Below, we consider the interconnection of $\Sigma_{1}, \Sigma_{2}$ where the communication between the subsystems is performed over multiple communication channels which are subject to constraints typical for serial communication networks, such as the existence of unknown time-varying possibly unbounded communication delays as well as possible packet losses. More precisely, consider the interconnection of $\Sigma_{1}, \Sigma_{2}$ whose inputs and outputs are related according to the formulas

$$
\mathbf{u}_{1}^{+}(t) \equiv 0, \quad \mathbf{u}_{2}^{+}(t) \equiv 0 \quad \text { for } t<T_{0}
$$


and

$$
\mathbf{u}_{1}^{+}(t) \leq \boldsymbol{\Psi}_{2}\left(\hat{\mathbf{y}}_{2}^{+}(t)\right), \mathbf{u}_{2}^{+}(t) \leq \boldsymbol{\Psi}_{1}\left(\hat{\mathbf{y}}_{1}^{+}(t)\right) \text { for } t \geq T_{0}
$$

where $T_{0} \in \mathbb{R}$ is the time instant when a connection between the subsystems has been established, $\boldsymbol{\Psi}_{1} \in \mathcal{G}^{p \times p}, \boldsymbol{\Psi}_{2} \in \mathcal{G}^{q \times q}$, and $\hat{\mathbf{y}}_{1}^{+}, \hat{\mathbf{y}}_{2}^{+}$are delayed versions of $\mathbf{y}_{1}^{+}$, $\mathbf{y}_{2}^{+}$, defined as follows

$$
\hat{\mathbf{y}}_{1}^{+}(t):=\left[\begin{array}{c}
\left|y_{1}^{\{1\}}\left(t-\tau_{f}^{\{1\}}(t)\right)\right| \\
\vdots \\
\left|y_{1}^{\{p\}}\left(t-\tau_{f}^{\{p\}}(t)\right)\right|
\end{array}\right], \quad \hat{\mathbf{y}}_{2}^{+}(t):=\left[\begin{array}{c}
\left|y_{2}^{\{1\}}\left(t-\tau_{b}^{\{1\}}(t)\right)\right| \\
\vdots \\
\left|y_{2}^{\{q\}}\left(t-\tau_{b}^{\{q\}}(t)\right)\right|
\end{array}\right] .
$$

The assumption below is imposed on communication delays $\tau_{f}^{\{i\}}, \tau_{b}^{\{j\}}$.

Assumption 1. The communication delays $\tau_{f}^{\{i\}}, \tau_{b}^{\{j\}}:\left[T_{0},+\infty\right) \rightarrow \mathbb{R}_{+}, i \in$ $\{1, \ldots, p\}, j \in\{1, \ldots, q\}$, are Lebesgue measured functions with the following properties:

i) there exist $\tau_{*}>0$ and a piecewise continuous function $\tau^{*}: \mathbb{R} \rightarrow \mathbb{R}_{+}$satisfying $\tau^{*}\left(t_{2}\right)-\tau^{*}\left(t_{1}\right) \leq t_{2}-t_{1}$, such that the inequalities

$$
\tau_{*} \leq \min _{\substack{i \in\{1, \ldots, p\} \\ j \in\{1, \ldots, q\}}}\left\{\tau_{f}^{\{i\}}(t), \tau_{b}^{\{j\}}(t)\right\} \leq \max _{\substack{i \in\{1, \ldots, p\} \\ j \in\{1, \ldots, q\}}}\left\{\tau_{f}^{\{i\}}(t), \tau_{b}^{\{j\}}(t)\right\} \leq \tau^{*}(t)
$$

hold for all $t \geq 0$;

ii)

$$
t-\max _{\substack{i \in\{1, \ldots, p\} \\ j \in\{1, \ldots, q\}}}\left\{\tau_{f}^{\{i\}}(t), \tau_{b}^{\{j\}}(t)\right\} \rightarrow+\infty \quad \text { as } \quad t \rightarrow+\infty
$$

Assumption 1 is not a restrictive one. In particular, part i) implies the existence of an upper bound $\tau^{*}(t)$ for delays $\tau_{f}^{\{i\}}(t), \tau_{b}^{\{j\}}(t)$, which is possibly a time-varying unbounded function of time that does not grow faster than the time itself. Note that the lower bound $\tau_{*}>0$ always exists in any practical (not necessarily networkedbased) feedback system due to natural transmission/propagation constraints. From mathematical point of view, however, the existence of a positive lower bound is a 
purely technical assumption which allows to simplify formulation and proof of the small-gain theorem, in particular, to avoid dealing with well-posedness issue. Assumption 1 , i) can always be satisfied in real world communication networks by using standard techniques such as sequence numbering and (or) timestamping. For example, a sequence number can be assigned to each outgoing packet at the sender side, while at the receiver side, the control signals are applied to the system's input in order according to their packet's sequence number. In this case, the packets with sequence numbers less than those already applied to the system's input are considered to be outdated and has to be discarded. As a result, the control signals are applied to the input in the same order as they were sent (possibly with some packets lost). Mathematically, this implies that $t-\tau(t)$, where $\tau(\cdot)$ is the corresponding communication delay, is a nondecreasing function of time $t$. Now, suppose we have $n$ communication channels with the corresponding communication delays $\tau_{i}(\cdot), i \in\{1, \ldots, n\}$, such that $t-\tau_{i}(t)$ are nondecreasing functions of time. Denote $\tau_{\max }(\cdot)=\max _{i} \tau_{i}(\cdot)$, then $\min _{i}\left(t-\tau_{i}(t)\right)=t-\tau_{\max }(t)$ is also a nondecreasing function of time, i.e., $t_{1}-\tau_{\max }\left(t_{1}\right) \leq t-\tau_{\max }(t)$ holds for all $t \geq t_{1}$. Rewriting the last inequality, we see that $\tau_{\max }(t)-\tau_{\max }\left(t_{1}\right) \leq t-t_{1}$ holds for all $t \geq t_{1}$, i.e., the Assumption 1,i) is satisfied with an upper bound $\tau^{*}(\cdot):=\tau_{\max }(\cdot)$.

On the other hand, Assumption 1 can also be satisfied by using timestamps and by assigning a maximal packet lifetime $T_{\max }<\infty$, so that all packets "older" than $T_{\max }$ are to be discarded on the receiver's side. In this case, Assumption 1, i) holds with $\tau^{*}(t):=t-T_{0}+\tau_{\max }\left(T_{0}\right)+T_{\max }$, where $\tau_{\max }(\cdot):=\max _{i} \tau_{i}(\cdot)$. To prove this, pick an arbitrary $t \geq T_{0}$. Suppose there are no packets arrived to the receiver over $i$-th channel during the time interval $\left[T_{0}, t\right]$, then we have $\tau_{i}(t) \leq t-T_{0}+\tau_{\max }\left(T_{0}\right)$. Otherwise, let $t_{1} \in\left[T_{0}, t\right]$ be the last time instant (within the interval $\left[T_{0}, t\right]$ ) when a new packet arrives to the receiver over $i$-th channel. Then, $\tau_{i}\left(t_{1}\right) \leq T_{\max }$, which 
implies that $\tau_{i}(t) \leq t-t_{1}+T_{\max } \leq t-T_{0}+T_{\max }$. In both these cases, we have $\tau_{i}(t) \leq t-T_{0}+\tau_{\max }\left(T_{0}\right)+T_{\max }:=\tau^{*}(t)$. Clearly, the function $\tau^{*}(\cdot)$ satisfies $\tau^{*}\left(t_{2}\right)-\tau^{*}\left(t_{1}\right)=t_{2}-t_{1}$, i.e, $\tau^{*}(\cdot)$ is an upper bound that satisfies Assumption 1, i).

Finally, for the above described schemes with time-stamping and (or) sequence numbering, part ii) of Assumption 1 simply means the existence of communication between the subsystems on a semi-infinite time interval. To clarify this statement, assume the converse, i.e., there exists a sequence $s_{j} \rightarrow+\infty, j=1,2, \ldots$, and $t_{L}<+\infty$ such that

$$
s_{j}-\tau_{i}\left(s_{j}\right) \leq t_{L}
$$

holds for some $i \in\{1, \ldots, n\}$ and for all $s_{j}$. For the scheme with sequence numbering, it is shown above that $t-\tau_{i}(t)$ is a nondecreasing function of $t$, therefore, (2.8) is equivalent to $\sup _{t \geq T_{0}}\left\{t-\tau_{i}(t)\right\} \leq t_{L}$. The last simply implies that the packets which are sent after $t_{L}$ would never be received by the other subsystem, i.e., no communication exists between the subsystems during the time interval $\left(t_{L}, \infty\right)$. For the scheme with timestamping, on the other hand, it is shown above that if a packet arrives to the receiver at some instant $t_{\text {arr }} \in\left[T_{0},+\infty\right)$ then

$$
\tau_{i}\left(s_{j}\right) \leq s_{j}-t_{a r r}+T_{\max }
$$

holds for all $s_{j} \geq t_{a r r}$. Combining (2.8) and (2.9), we see that $t_{a r r} \leq t_{L}+T_{\max }$, i.e., no packets would arrive to the receiver after $t=t_{L}+T_{\max }$. Overall, Assumption 1, ii) is always satisfied unless the communication between the subsystems is totally lost on a semi-infinite time interval. 


\subsection{Small-Gain Theorem}

The stability properties of the interconnection of $\Sigma_{1}$ and $\Sigma_{2}$ are addressed in this work under the assumption that both the subsystems are IOS. Below, this assumption is presented formally.

Assumption 2. The systems $\Sigma_{1}, \Sigma_{2}$ are IOS with restrictions $\Delta_{x i}, \Delta_{u i}, \Delta_{w i}$ and offsets $\delta_{i}, i \in\{1,2\}$, respectively, where $\Delta_{x 1}, \Delta_{x 2} \in \mathbb{R}_{+}, \Delta_{u 1} \in \mathbb{R}_{+}^{q}, \Delta_{u 2} \in \mathbb{R}_{+}^{p}$, $\delta_{1} \in \mathbb{R}_{+}^{p}, \delta_{2} \in \mathbb{R}_{+}^{q}$, and $\Delta_{w i} \in \mathbb{R}_{+}^{v_{i}}, i \in\{1,2\}$. More precisely, there exist $\beta_{1} \in \mathcal{K}_{\infty}^{p \times 1}$, $\beta_{2} \in \mathcal{K}_{\infty}^{q \times 1}, \Gamma_{1 u} \in \mathcal{G}^{p \times q}, \Gamma_{2 u} \in \mathcal{G}^{q \times p}, \Gamma_{1 w} \in \mathcal{G}^{p \times v_{1}}$, and $\Gamma_{2 w} \in \mathcal{G}^{q \times v_{2}}$, such that for each $i \in\{1,2\}$ and each $t_{0} \in \mathbb{R}$, the conditions $\left|x_{i}\left(t_{0}\right)\right| \leq \Delta_{x i}, \sup _{t \geq t_{0}} \mathbf{u}_{i}^{+} \leq \Delta_{u i}$, and sup $\mathbf{w}_{i}^{+} \leq \Delta_{w i}$ imply that the corresponding solution of $\Sigma_{i}$ is well-defined for all $t \geq t_{0}$ $t \in\left[t_{0},+\infty\right)$, and the following inequalities hold

$$
\begin{aligned}
& \sup _{t \geq t_{0}} \mathbf{y}_{i}^{+} \leq \max \left\{\beta_{i}\left(\left|x_{i d}\left(t_{0}\right)\right|\right), \sup _{t \geq t_{0}} \boldsymbol{\Gamma}_{i u}\left(\mathbf{u}_{i d}^{+}\right), \sup _{t \geq t_{0}} \boldsymbol{\Gamma}_{i w}\left(\mathbf{w}_{i d}^{+}\right), \delta_{i}\right\}, \\
& \limsup _{t \rightarrow+\infty} \mathbf{y}_{i}^{+} \leq \max \left\{\limsup _{t \rightarrow+\infty} \Gamma_{i u}\left(\mathbf{u}_{i d}^{+}\right), \limsup _{t \rightarrow+\infty} \Gamma_{i w}\left(\mathbf{w}_{i d}^{+}\right), \delta_{i}\right\} . \bullet
\end{aligned}
$$

Remark 3. The form of Assumption 2 (i.e., the stability with finite restrictions and a nonzero offset), in particular, allows the subsystem to be implemented as a sampled-data system with quantization. Indeed, for a nonlinear sampled-data system with quantization, the global stability cannot generally be achieved. On the contrary, the existing design methods (such as the controller emulation approach as well as the design based on approximate discrete-time models) generally lead to stability with some finite restrictions and some nonzero offset [59], which exactly meets the requirements imposed by Assumption 2.

Below, we use the notation $\mathbf{x}^{+}:=\left(\left|x_{1}\right|,\left|x_{2}\right|\right)^{T} \in \mathbb{R}_{+}^{2}, \mathbf{y}^{+}:=\left(\mathbf{y}_{1}^{+T}, \mathbf{y}_{2}^{+T}\right)^{T} \in$ $\mathbb{R}_{+}^{p+q}$, and $\mathbf{w}^{+}:=\left(\mathbf{w}_{1}^{+T}, \mathbf{w}_{2}^{+T}\right)^{T} \in \mathbb{R}_{+}^{v_{1}+v_{2}}$. The following small gain theorem is the main result of this section. 
Theorem 1. Suppose the system (2.2) - (2.5) satisfies Assumptions 1, 2, and there exist $\delta, \Delta \in \mathbb{R}_{+}^{p}, \mathbf{0} \leq \delta<\Delta$, such that the following small gain condition holds

$$
\Gamma_{1 u} \circ \Psi_{2} \circ \Gamma_{2 u} \circ \Psi_{1}(\mathrm{~s})<\mathrm{s} \quad \text { for all } \mathrm{s} \in(\delta, \Delta)
$$

If $\Delta>\Delta^{*}$, where

$$
\Delta^{*}:=\max \left\{\beta_{1}\left(\Delta_{x 1}\right), \boldsymbol{\Gamma}_{1 u} \circ \mathbf{\Psi}_{2} \circ \beta_{2}\left(\Delta_{x 2}\right), \boldsymbol{\Gamma}_{1 u} \circ \boldsymbol{\Psi}_{2} \circ \Gamma_{2 w}\left(\Delta_{w 2}\right), \boldsymbol{\Gamma}_{1 w}\left(\Delta_{w 1}^{+}\right), \delta\right\},
$$

then the system (2.2) - (2.5), is IOS at $t=T_{0}$ in the sense of Definition 1 with

$$
t_{d}\left(T_{0}\right):=t_{1 d}\left(T_{0}\right)+t_{2 d}\left(T_{0}\right)+\tau^{*}\left(T_{0}\right)+\tau^{*}\left(T_{0}-\tau^{*}\left(T_{0}\right)\right)
$$

restrictions $\Delta_{x}:=\left(\Delta_{x 1}, \Delta_{x 2}\right)^{T} \in \mathbb{R}_{+}^{2}, \Delta_{w}:=\left(\Delta_{w 1}^{T}, \Delta_{w 2}^{T}\right)^{T} \in \mathbb{R}_{+}^{v_{1}+v_{2}}$, and offset $\delta_{0}:=\left(\delta^{T},\left(\boldsymbol{\Gamma}_{2 u} \circ \boldsymbol{\Psi}_{1}(\delta)\right)^{T}\right)^{T} \in \mathbb{R}_{+}^{p+q}$. More precisely, the conditions

$$
\mathbf{x}_{d}^{+}\left(T_{0}\right) \leq \Delta_{x}, \quad \sup _{t \geq T_{0}} \mathbf{w}_{d}^{+} \leq \Delta_{w}
$$

imply that the following inequalities hold

$$
\begin{gathered}
\sup _{t \geq T_{0}} \mathbf{y}^{+} \leq \max \left\{\beta\left(\mathbf{x}_{d}^{+}\left(T_{0}\right)\right), \boldsymbol{\Gamma}_{w}\left(\sup _{t \geq T_{0}} \mathbf{w}_{d}^{+}\right), \delta_{0}\right\} \\
\limsup _{t \rightarrow+\infty} \mathbf{y}^{+} \leq \max \left\{\boldsymbol{\Gamma}_{w}\left(\limsup _{t \rightarrow+\infty} \mathbf{w}_{d}^{+}\right), \delta_{0}\right\},
\end{gathered}
$$

where $\beta \in \mathcal{G}^{(p+q) \times 2}$ and $\Gamma_{w} \in \mathcal{G}^{(p+q) \times\left(v_{1}+v_{2}\right)}$ are defined as follows

$$
\begin{gathered}
\beta:=\left[\begin{array}{cc}
\beta_{1} & \boldsymbol{\Gamma}_{1 u} \circ \boldsymbol{\Psi}_{2} \circ \beta_{2} \\
\boldsymbol{\Gamma}_{2 u} \circ \boldsymbol{\Psi}_{1} \circ \beta_{1} & \beta_{2}
\end{array}\right], \\
\boldsymbol{\Gamma}_{w}:=\left[\begin{array}{cc}
\boldsymbol{\Gamma}_{1 w} & \boldsymbol{\Gamma}_{1 u} \circ \boldsymbol{\Psi}_{2} \circ \boldsymbol{\Gamma}_{2 w} \\
\boldsymbol{\Gamma}_{2 u} \circ \boldsymbol{\Psi}_{1} \circ \boldsymbol{\Gamma}_{1 w} & \boldsymbol{\Gamma}_{2 w}
\end{array}\right] .
\end{gathered}
$$




\subsubsection{Proof of Theorem 1}

Consider the system (2.2) - (2.5), and suppose

$$
\left|x_{1 d}\left(T_{0}\right)\right| \leq \Delta_{x 1},\left|x_{2 d}\left(T_{0}\right)\right| \leq \Delta_{x 2}, \sup _{t \in\left[T_{0},+\infty\right)}\left|\mathbf{w}_{1 d}^{+}\right| \leq \Delta_{w 1}, \sup _{t \in\left[T_{0},+\infty\right)}\left|\mathbf{w}_{2 d}^{+}\right| \leq \Delta_{w 2},
$$

where $\Delta_{x 1}, \Delta_{x 2}, \Delta_{w 1}, \Delta_{w 2}$ satisfy (2.13). Since $\mathbf{u}_{1}^{+}(t) \equiv \mathbf{0}$, and $\mathbf{u}_{2}^{+}(t) \equiv \mathbf{0}$ for $t<T_{0}$, Assumption 2 together with (2.18) as well as causality arguments imply that

$$
\begin{aligned}
\mathbf{y}_{i d}^{+}\left(T_{0}\right) & \leq \max \left\{\beta_{i}\left(\left|x_{i d}\left(T_{0}\right)\right|\right), \Gamma_{i w}\left(\mathbf{w}_{i d}^{+}\left(T_{0}\right)\right), \delta_{i}\right\} \\
& \leq \max \left\{\beta_{i}\left(\Delta_{x i}\right), \Gamma_{i w}\left(\Delta_{w i}\right), \delta_{i}\right\}
\end{aligned}
$$

holds for $i \in\{1,2\}$. Taking into account (2.3), (2.4), as well as Assumption 1, i), we see that

$$
\begin{aligned}
& \sup _{t \in\left[T_{0}-t_{d}\left(T_{0}\right), T_{0}+\tau_{*}\right]} \mathbf{u}_{1}^{+} \leq \boldsymbol{\Psi}_{2}\left(\max \left\{\beta_{2}\left(\Delta_{x 2}\right), \boldsymbol{\Gamma}_{2 w}\left(\Delta_{w 2}\right)\right\}\right), \\
& \sup _{t \in\left[T_{0}-t_{d}\left(T_{0}\right), T_{0}+\tau_{*}\right]} \mathbf{u}_{2}^{+} \leq \boldsymbol{\Psi}_{1}\left(\max \left\{\beta_{1}\left(\Delta_{x 1}\right), \boldsymbol{\Gamma}_{1 w}\left(\Delta_{w 1}\right)\right\}\right),
\end{aligned}
$$

which implies that all the inputs of the system (2.2) - (2.5) are uniformly essentially bounded on $\left[T_{0}-t_{d}\left(T_{0}\right), T_{0}+\tau_{*}\right]$. Due to the IOS assumptions imposed on the subsystems, this implies that the solutions of (2.2) - (2.5) are well-defined for all $t \in\left[T_{0}, T_{\max }\right)$, where $T_{\max }>T_{0}+\tau_{*}>T_{0}$. Furthernore, the following inequality holds

$$
\sup _{t \in\left[T_{0}, T_{\max }\right)} \mathbf{y}_{1 d}^{+} \leq \Delta^{*}
$$

where $\Delta^{*}<\Delta$ is defined by (2.13). To prove (2.20), assume the converse. Then there exists $T_{1} \in\left[T_{0}, T_{\max }-\tau_{*}\right.$ ) such that

$$
\sup _{t \in\left[T_{0}, T_{1}\right]} \mathbf{y}_{1 d}^{+} \leq \Delta^{*}
$$

and

$$
\sup _{t \in\left[T_{0}, T_{1}+\tau_{*}\right]} \mathbf{y}_{1}^{+}>\Delta^{*} .
$$


However, from (2.10), (2.14), and (2.18), together with Assumption 1, i), we see that

$\sup _{t \in\left[T_{0}, T_{0}+T_{*}\right]} \mathbf{y}_{1}^{+} \leq \max \left\{\begin{array}{c}\beta_{1}\left(\Delta_{x 1}\right), \boldsymbol{\Gamma}_{1 w}\left(\Delta_{w 1}\right), \boldsymbol{\Gamma}_{1 u} \circ \boldsymbol{\Psi}_{2} \circ \beta_{2}\left(\Delta_{x 2}\right), \\ \Gamma_{1 u} \circ \boldsymbol{\Psi}_{2} \circ \boldsymbol{\Gamma}_{2 w}\left(\Delta_{w 2}\right), \boldsymbol{\Gamma}_{1 u} \circ \boldsymbol{\Psi}_{2} \circ \boldsymbol{\Gamma}_{2 u} \circ \boldsymbol{\Psi}_{1}\left(\sup _{t \in\left[T_{0}, T_{1}\right]} \mathbf{y}_{1 d}^{+}\right)\end{array}\right\}$.

Taking into account (2.12), (2.13), (2.21), we get

$$
\sup _{t \in\left[T_{0}, T_{1}+\tau_{*}\right]} \mathbf{y}_{1}^{+} \leq \max \left\{\begin{array}{c}
\beta_{1}\left(\Delta_{x 1}\right), \boldsymbol{\Gamma}_{1 w}\left(\Delta_{w 1}\right), \Gamma_{1 u} \circ \mathbf{\Psi}_{2} \circ \beta_{2}\left(\Delta_{x 2}\right), \\
\boldsymbol{\Gamma}_{1 u} \circ \mathbf{\Psi}_{2} \circ \boldsymbol{\Gamma}_{2 w}\left(\Delta_{w 2}\right), \Delta_{*}
\end{array}\right\}:=\Delta_{*},
$$

which contradicts (2.22). This contradiction proves (2.20).

Next, let us show that $T_{\max }=+\infty$. To prove this, assume the converse, i.e., $T_{\max }<+\infty$. Since both the subsystems are assumed to be IOS, the last implies that

$$
\sup _{t \in\left[T_{0}, T_{\max }\right)} \max \left\{\mathbf{u}_{1}^{+}(t), \mathbf{u}_{2}^{+}(t)\right\}=+\infty
$$

which may only be possible if

$$
\sup _{t \in\left[T_{0}, T_{\max }-\tau_{*}\right)} \max \left\{\mathbf{y}_{1}^{+}(t), \mathbf{y}_{2}^{+}(t)\right\}=+\infty .
$$

Combining (2.10) with $(2.3),(2.4)$, it is easy to see that (2.24) necessarily implies that $\sup _{t \in\left[T_{0}, T_{\max }-\tau_{*}\right)} \mathbf{y}_{1}^{+}(t)=+\infty$, which contradicts $(2.20)$. Thus, $T_{\max }=+\infty$. In particular, $(2.20)$ becomes

$$
\sup _{t \in\left[T_{0},+\infty\right)} \mathbf{y}_{1 d}^{+} \leq \Delta^{*},
$$

Now, the proof can be easily finalized as follows. Combining (2.10) with (2.4)(2.5), (2.19), and using Assumption 1, i), one gets

$$
\sup _{t \geq T_{0}} \mathbf{y}_{1 d}^{+} \leq \max \left\{\begin{array}{c}
\beta_{1}\left(\left|x_{1 d}\left(T_{0}\right)\right|\right), \boldsymbol{\Gamma}_{1 w}\left(\sup _{t \geq T_{0}} \mathbf{w}_{1 d}^{+}(t)\right), \boldsymbol{\Gamma}_{1 u} \circ \mathbf{\Psi}_{2} \circ \beta_{2}\left(\left|x_{2 d}\left(T_{0}\right)\right|\right), \\
\boldsymbol{\Gamma}_{1 u} \circ \boldsymbol{\Psi}_{2} \circ \boldsymbol{\Gamma}_{2 w}\left(\sup _{t \geq T_{0}} \mathbf{w}_{2 d}^{+}(t)\right), \boldsymbol{\Gamma}_{1 u} \circ \boldsymbol{\Psi}_{2} \circ \boldsymbol{\Gamma}_{2 u} \circ \boldsymbol{\Psi}_{1}\left(\sup _{t \geq T_{0}} \mathbf{y}_{1 d}^{+}\right)
\end{array}\right\},
$$


and taking into account (2.25) and the small-gain condition (2.12), it is easy to see that

$$
\sup _{t \geq T_{0}} \mathbf{y}_{1 d}^{+} \leq \max \left\{\begin{array}{c}
\beta_{1}\left(\left|x_{1 d}\left(T_{0}\right)\right|\right), \boldsymbol{\Gamma}_{1 w}\left(\sup _{t \geq T_{0}} \mathbf{w}_{1 d}^{+}(t)\right), \\
\boldsymbol{\Gamma}_{1 u} \circ \mathbf{\Psi}_{2} \circ \beta_{2}\left(\left|x_{2 d}\left(T_{0}\right)\right|\right), \boldsymbol{\Gamma}_{1 u} \circ \mathbf{\Psi}_{2} \circ \boldsymbol{\Gamma}_{2 w}\left(\sup _{t \geq T_{0}} \mathbf{w}_{2 d}^{+}(t)\right), \delta
\end{array}\right\}
$$

On the other hand, combining (2.10), (2.3), (2.4) with (2.27), and taking into account (2.19), we get

$$
\sup _{t \geq T_{0}} \mathbf{y}_{2}^{+} \leq \max \left\{\begin{array}{c}
\beta_{2}\left(\left|x_{2 d}\left(t_{0}\right)\right|\right), \boldsymbol{\Gamma}_{2 u} \circ \boldsymbol{\Psi}_{1} \circ \beta_{1}\left(\left|x_{1 d}\left(T_{0}\right)\right|\right), \\
\boldsymbol{\Gamma}_{2 u} \circ \boldsymbol{\Psi}_{1} \circ \boldsymbol{\Gamma}_{1 u} \circ \boldsymbol{\Psi}_{2} \circ \beta_{2}\left(x_{2}\left(T_{0}\right)\right), \boldsymbol{\Gamma}_{2 u} \circ \boldsymbol{\Psi}_{1} \circ \boldsymbol{\Gamma}_{1 w}\left(\sup _{t \geq T_{0}} \mathbf{w}_{1 d}^{+}\right) \\
\boldsymbol{\Gamma}_{2 u} \circ \boldsymbol{\Psi}_{1} \circ \boldsymbol{\Gamma}_{1 u} \circ \boldsymbol{\Psi}_{2} \circ \boldsymbol{\Gamma}_{2 w}\left(\sup _{t \geq T_{0}} \mathbf{w}_{2 d}^{+}\right), \boldsymbol{\Gamma}_{2 w}\left(\sup _{t \geq T_{0}} \mathbf{w}_{2 d}^{+}\right)
\end{array}\right\}
$$

Under the assumptions of the theorem, however, we have

$$
\boldsymbol{\Gamma}_{2 u} \circ \boldsymbol{\Psi}_{1} \circ \boldsymbol{\Gamma}_{1 u} \circ \boldsymbol{\Psi}_{2} \circ \beta_{2}\left(\left|x_{2 d}\left(t_{0}\right)\right|\right) \leq \max \left\{\beta_{2}\left(\left|x_{2 d}\left(t_{0}\right)\right|\right), \boldsymbol{\Gamma}_{2 u} \circ \Psi_{1}(\delta)\right\} .
$$

Indeed, note first that the small gain condition (2.12) imply

$$
\Gamma_{2 u} \circ \Psi_{1} \circ \Gamma_{1 u} \circ \Psi_{2}(\mathrm{~s})<\mathrm{s}
$$

for all $\mathbf{s} \in\left(\boldsymbol{\Gamma}_{2 u} \circ \boldsymbol{\Psi}_{1}(\delta), \boldsymbol{\Gamma}_{2 u} \circ \boldsymbol{\Psi}_{1}(\Delta)\right)$. Also, (2.13) imply that

$$
\Gamma_{2 u} \circ \Psi_{1} \circ \Gamma_{1 u} \circ \Psi_{2} \circ \beta_{2}\left(\left|x_{2 d}\left(t_{0}\right)\right|\right) \leq \Gamma_{2 u} \circ \Psi_{1}\left(\Delta^{*}\right):=\Delta_{1}^{*} .
$$

Now, if $\beta_{2}\left(\left|x_{2 d}\left(t_{0}\right)\right|\right) \leq \Delta_{1}^{*}$, then (2.28) follows from (2.29). Otherwise (i.e., if $\left.\beta_{2}\left(\left|x_{2 d}\left(t_{0}\right)\right|\right)>\Delta_{1}^{*}\right)$, the inequality (2.28) follows from (2.30). Analogously,

$$
\Gamma_{2 u} \circ \boldsymbol{\Psi}_{1} \circ \Gamma_{1 u} \circ \boldsymbol{\Psi}_{2} \circ \boldsymbol{\Gamma}_{2 w}\left(\sup _{t \geq T_{0}} \mathbf{w}_{2 d}^{+}\right) \leq \max \left\{\boldsymbol{\Gamma}_{2 w}\left(\sup _{t \geq T_{0}} \mathbf{w}_{2 d}^{+}\right), \Gamma_{2 u} \circ \boldsymbol{\Psi}_{1}(\delta)\right\}
$$

Taking into account (2.28), (2.31), we get

$$
\sup _{t \geq T_{0}} \mathbf{y}_{2}^{+} \leq \max \left\{\begin{array}{c}
\beta_{2}\left(\left|x_{2 d}\left(T_{0}\right)\right|\right), \Gamma_{2 u} \circ \boldsymbol{\Psi}_{1} \circ \beta_{1}\left(\left|x_{1 d}\left(T_{0}\right)\right|\right), \\
\Gamma_{2 u} \circ \boldsymbol{\Psi}_{1} \circ \boldsymbol{\Gamma}_{1 w}\left(\sup _{t \geq T_{0}} \mathbf{w}_{1 d}^{+}\right), \boldsymbol{\Gamma}_{2 w}\left(\sup _{t \geq T_{0}} \mathbf{w}_{2 d}^{+}\right), \boldsymbol{\Gamma}_{2 u} \circ \boldsymbol{\Psi}_{1}(\delta)
\end{array}\right\} .
$$


Finally, combining (2.27) and (2.32), and using the notation (2.17), we get (2.15).

To prove convergence, note that Assumption 1, ii), implies that

$$
\limsup _{t \rightarrow+\infty} \mathbf{u}_{1}^{+} \leq \limsup _{t \rightarrow+\infty} \boldsymbol{\Psi}_{2}\left(\mathbf{y}_{2}^{+}\right), \quad \limsup _{t \rightarrow+\infty} \mathbf{u}_{2}^{+} \leq \limsup _{t \rightarrow+\infty} \boldsymbol{\Psi}_{\mathbf{1}}\left(\mathbf{y}_{1}^{+}\right)
$$

Substituting the above inequalities into (2.11) and taking into account (2.12), (2.25), we get

$$
\limsup _{t \rightarrow+\infty} \mathbf{y}_{2}^{+} \leq \max \left\{\boldsymbol{\Gamma}_{2 u} \circ \boldsymbol{\Psi}_{1} \circ \boldsymbol{\Gamma}_{1 w}\left(\limsup _{t \rightarrow+\infty} \mathbf{w}_{1 d}^{+}\right), \boldsymbol{\Gamma}_{2 w}\left(\limsup _{t \rightarrow+\infty} \mathbf{w}_{2 d}^{+}\right), \boldsymbol{\Gamma}_{2 u} \circ \boldsymbol{\Psi}_{1}(\delta)\right\} .
$$

The convergence of $\mathbf{y}_{2}^{+}$can be shown analogously. This completes the proof of Theorem 1.

\subsection{Example}

The IOS small gain theorem presented above will serve as the main tool for stability analysis of the network-based teleoperator schemes described in the next two Chapters. The purpose of the following very simple example is merely to illustrate that the proposed multi-channel version of the small-gain theorem may indeed lead to less conservative stability conditions comparing to the more traditional "onechannel-in-each-direction" approach. Consider a teleoperator system which consists of a master and a slave manipulators connected through a communication channel. A human operator moves the master, and the information about the master trajectory is sent to the remotely located slave. The slave is designed to follow the motion of the master. When slave is in contact with the environment, the information about contact forces is sent back to the master and applied to master's motor to make the human operator feel the interaction. Suppose the master manipulator is equipped with a local "PD+gravity compensation" control algorithm; the closed-loop master 
subsystem is then described by the following equations

$$
H_{m}\left(q_{m}\right) \ddot{q}_{m}+C_{m}\left(q_{m}, \dot{q}_{m}\right) \dot{q}_{m}+K_{m}\left(\dot{q}_{m}+\Lambda_{m} q_{m}\right)=f_{h}+\hat{f}_{e}
$$

where $q_{m}$ is the position of the master, $H_{m}\left(q_{m}\right), C_{m}\left(q_{m}, \dot{q}_{m}\right)$ are the matrices of inertia and Coriolis/centrifugal forces, $K_{m}=K_{m}^{T}>0, \Lambda_{m}=\Lambda_{m}^{T}>0$ are controller parameters, $f_{h}$ is the force applied by the human, and $\hat{f}_{e}$ is the force reflection term. It is easy to check (for details, see [60]) that the system is ISS, and the gain from $f_{h}$ to $\left(q_{m}^{T}, \dot{q}_{m}^{T}\right)^{T}$ (denoted by $\gamma_{\left[f_{e} \rightarrow\left(q_{m}, \dot{q}_{m}\right)\right]} \in \mathcal{K}$ ) can be made "arbitrarily small" by an appropriate choice of $K_{m}, \Lambda_{m}$; however, the gain from $\hat{f}_{e}$ to the "output" $\ddot{q}_{m}$ (denoted by $\gamma_{\left[f_{e} \rightarrow \ddot{q}_{m}\right]} \in \mathcal{K}$ ) cannot be assigned arbitrarily. On the other hand, let the "slave+environment" interconnection be described as follows

$$
\begin{aligned}
& \dot{x}_{s}=F_{s}\left(x_{s}, \hat{q}_{m}, \hat{\dot{q}}_{m}, \hat{\vec{q}}_{m}\right), \\
& f_{e}=G_{s}\left(x_{s}, \hat{q}_{m}, \hat{\dot{q}}_{m}, \hat{\vec{q}}_{m}\right),
\end{aligned}
$$

where $x_{s}$ is a state of the "slave+environment" subsystem, $\hat{q}_{m}, \hat{\dot{q}}_{m}, \hat{\vec{q}}_{m}$ are delayed versions of the master position, velocity, and acceleration, and $f_{e}$ is the contact force due to environment which plays the role of the output of the subsystem; $f_{e}$ is then sent over the communication channel, and its delayed version $\hat{f}_{e}$ is applied to the motors of the master. (Normally, $\hat{\dot{q}}_{m}, \hat{\ddot{q}}_{m}$ are recovered from $\hat{q}_{m}$ using some sort of estimation/filtering process rather than directly sent over the communication channel, but this is not important for our purposes). Suppose the control system is designed on the slave side such that the "slave+environment" subsystem is ISS. In most situations, the system can be designed such that the gain from $\hat{\ddot{q}}_{m}$ to $f_{e}$ (denoted by $\gamma_{\left[\hat{\vartheta}_{m} \rightarrow f_{e}\right]} \in \mathcal{K}$ ) can be made negligible (arbitrarily small), however, the gain $\gamma_{\left[\left(\hat{q}_{m}, \hat{q}_{m}\right) \rightarrow f_{e}\right]} \in \mathcal{K}$ significantly depends on environmental damping/stiffness, and cannot be made small without deterioration of the slave's tracking properties. For this system, using traditional approach (one gain that works for all input/output 
pairs) one gets the following small gain condition

$$
\max \left\{\gamma_{\left[\left(\hat{q}_{m}, \hat{q}_{m}\right) \rightarrow f_{e}\right]}, \gamma_{\left[\hat{\tilde{q}}_{m} \rightarrow f_{e}\right]}\right\} \circ \max \left\{\gamma_{\left[f_{e} \rightarrow\left(q_{m}, \dot{q}_{m}\right)\right]}, \gamma_{\left[f_{e} \rightarrow \ddot{q}_{m}\right]}\right\}(s)<s
$$

which is not satisfied if $\gamma_{\left[\left(\hat{q}_{m}, \hat{q}_{m}\right) \rightarrow f_{e}\right]} \circ \gamma_{\left[f_{e} \rightarrow \ddot{q}_{m}\right]}$ is not a strict contraction. Thus, in this case the traditional form of the IOS small gain theorem does not guarantee stability. On the other hand, using "multi-channel" approach proposed, one gets the small gain condition of the form

$$
\begin{gathered}
{\left[\gamma_{\left[\left(\hat{q}_{m}, \hat{\hat{q}}_{m}\right) \rightarrow f_{e}\right]} \gamma_{\left[\hat{\hat{q}}_{m} \rightarrow f_{e}\right]}\right] \circ\left[\begin{array}{c}
\gamma_{\left[f_{e} \rightarrow\left(q_{m}, \dot{q}_{m}\right)\right]} \\
\gamma_{\left[f_{e} \rightarrow \ddot{q}_{m}\right]}
\end{array}\right](s)} \\
:=\max \left\{\gamma_{\left[\left(\hat{q}_{m}, \hat{\hat{q}}_{m}\right) \rightarrow f_{e}\right]} \circ \gamma_{\left[f_{e} \rightarrow\left(q_{m}, \dot{q}_{m}\right)\right]}, \gamma_{\left[\hat{\hat{q}}_{m} \rightarrow f_{e}\right]} \circ \gamma_{\left[f_{e} \rightarrow \ddot{q}_{m}\right]}\right\}(s)<s,
\end{gathered}
$$

which can always be satisfied (possibly with some restriction and offset) by choosing $\gamma_{\left[f_{e} \rightarrow\left(q_{m}, \dot{q}_{m}\right)\right]}, \gamma_{\left[\hat{\tilde{q}}_{m} \rightarrow f_{e}\right]} \in \mathcal{K}$ "sufficiently small". Thus, the multi-channel version of the small gain theorem guarantees stability, while the traditional version does not.

\subsection{Conclusion}

In this Chapter, we have presented a version of the IOS small gain theorem that is designed specifically for stability analysis of the interconnections where the communication between subsystems is performed asynchronously over multiple channels and is subject to constraints typical for communication networks. In particular, it allows to handle multiple time-varying possibly discontinuous and unbounded communication delays. The assumptions imposed on communication delays can always be satisfied in real world communication networks using techniques such as timestamping and (or) sequence numbering. The presented version of the IOS small-gain theorem will be applied later to the stability analysis of the force-reflecting teleoperator schemes. 


\section{Chapter 3}

\section{Small-Gain Based Schemes for Stable Bilateral Teleoperation}

\subsection{Introduction}

In this Chapter, we present schemes for bilateral teleoperation over networks that are based directly on the IOS/ISS small-gain result presented in Chapter 2. Schemes with general type of force-reflection term are considered; more precisely, the forcereflection term is assumed to be proportional to a (possibly trivial) linear combination of the position error and velocity error on the slave side as well as the actual contact force due to environment. Two types of control algorithms are proposed that guarantee stable contact with the remote environment in the presence of timevarying, discontinuous and possibly unbounded communication delays; also, arbitrarily precise tracking can be achieved if the communication process in the forward communication channel guarantees sufficiently smooth transmission of the master's trajectories. The theoretical results presented in this Chapter are supported by experiments. 
The Chapter is organized as follows. A mathematical model of the network-based teleoperator system is described in Section 3.2. In Section 3.3, control schemes for stable force-reflecting teleoperation are presented. Force reflection scheme is presented in Section 3.4, and the assumptions imposed on environmental dynamics are described in Section 3.5. Section 3.6 presents stability results, while tracking properties are studied in Section 3.7. In Section 3.8, proofs of the stability/tracking results are given. Experimental results are presented in Section 3.9. Finally, some concluding remarks are given.

\subsection{Mathematical Model of The Teleoperator}

\section{System}

\subsubsection{Master and Slave Manipulators}

Throughout this chapter, we consider a teleoperator system that consists of a master and a slave manipulators described by the following set of Euler-Lagrange equations

$$
\begin{gathered}
H_{m}\left(q_{m}\right) \ddot{q}_{m}+C_{m}\left(q_{m}, \dot{q}_{m}\right) \dot{q}_{m}+G_{m}\left(q_{m}\right)=u_{m}+f_{h}-\hat{f}_{r}, \\
H_{s}\left(q_{s}\right) \ddot{q}_{s}+C_{s}\left(q_{s}, \dot{q}_{s}\right) \dot{q}_{s}+G_{s}\left(q_{s}\right)=u_{s}-f_{e} .
\end{gathered}
$$

Here, $q_{m} \in \mathbb{Q}_{m}, q_{s} \in \mathbb{Q}_{s}$ are positions of the master and the slave manipulators, respectively, which belong to compact connected configuration manifolds $\mathbb{Q}_{m}, \mathbb{Q}_{s}$. For simplicity, assume $\mathbb{Q}_{m}=\mathbb{Q}_{s} \subset \mathbb{R}^{n}$. In the above equations, $H_{i}\left(q_{i}\right) \in \mathbb{R}^{n \times n}$ are matrices of inertia, $C_{i}\left(q_{i}, \dot{q}_{i}\right) \in \mathbb{R}^{n \times n}$ are matrices of Coriolis/centrifugal forces, and $G_{i}\left(q_{i}\right) \in \mathbb{R}^{n}$ are vectors of potential forces of the master $(i=m)$ and the slave $(i=s)$ manipulators, respectively. Also, $f_{h} \in \mathbb{R}^{n}$ is the force (torque) applied by the human operator to the master, $f_{e} \in \mathbb{R}^{n}$ is the environmental force (torque) applied 
to the slave, $\hat{f}_{r} \in \mathbb{R}^{n}$ is the force reflection term on the master side, and $u_{m}, u_{s} \in \mathbb{R}^{n}$ are the control inputs of the master and the slave respectively. The dynamics of the master (3.1) and the slave (3.2) manipulators are assumed to satisfy a set of standard properties described, for example, in [61, Section 2.1]

\subsubsection{Communication Constraints}

In this thesis, we generally address the situation where the communication between the master and the slave manipulators is subject to network-induced communication constraints. Following the considerations presented in Chapter 2, these constraints are described in terms of time-varying discontinuous and possibly unbounded communication delays. It is also assumed that the communication between the parts of telerobot is subject to an error that is due to quantization as well as measurement noise. Thus, let $\tau_{f}, \tau_{b}: \mathbb{R}_{+} \rightarrow \mathbb{R}_{+}$be the communication delays in the forward (from master to slave) and the backward (from slave to master) communication channels, respectively. Specifically, we assume that the master's position is transmitted to the slave side with communication delay $\tau_{f}(t)$ and is subject to quantization/measurement noise error $\hat{\delta}_{q}(t)$, i.e., the signal

$$
\hat{q}_{m}(t):=q_{m}\left(t-\tau_{f}(t)\right)+\hat{\delta}_{q}(t)
$$

is available for use in the control algorithm on the slave side. Analogously, a force reflection signal $f_{r}$ is transmitted back from the slave to the master side with communication delay $\tau_{b}(t)$, and is some subject to quantization/measurement noise error $\hat{\delta}_{f}(t)$, so that the signal

$$
\hat{f}_{r}:=f_{r}\left(t-\tau_{b}(t)\right)+\hat{\delta}_{f}(t)
$$

can be used for the force reflection on the master's side, where $f_{r}$ is the force reflection signal measured on the slave side. In general, it is assumed below that 
communication delays $\tau_{f}(\cdot), \tau_{b}(\cdot)$ satisfy Assumption 1; however, when dealing with tracking properties of the teleoperator system, some additional "smoothness" assumptions will be imposed on the forward communication delay $\tau_{f}(\cdot)$. Signals $\hat{\delta}_{q}(t), \hat{\delta}_{f}(t)$ are assumed to be essentially bounded uniformly for all $t \in \mathbb{R}$.

\subsection{Control Schemes}

The following control schemes are utilized. For the master manipulator, we will consider two types of control algorithm. The first control law utilizes the well known "PD+gravity compensation" control strategy

$$
u_{m}=G_{m}\left(q_{m}\right)-K_{m}\left(\dot{q}_{m}+\Lambda_{m} q_{m}\right)
$$

where $K_{m}, \Lambda_{m} \in \mathbb{R}^{n \times n}$ are symmetric (i.e., $K_{m}=K_{m}^{T}, \Lambda_{m}=\Lambda_{m}^{T}$ ) positive definite (i.e., $x^{T} K_{m} x>0, x^{T} \Lambda_{m} x>0$ for all $x \in \mathbb{R}^{n} \backslash\{0\}$ ) matrices. On the other hand, the following more detailed version of control algorithm

$$
u_{m}=-H_{m}\left(q_{m}\right) \Lambda_{m} \dot{q}_{m}-C_{m}\left(q_{m}, \dot{q}_{m}\right) \Lambda_{m} q_{m}+G_{m}\left(q_{m}\right)-K_{m}\left(\dot{q}_{m}+\Lambda_{m} q_{m}\right)
$$

will also be taken into consideration. The control algorithm for the slave manipulator has a form

$$
\begin{aligned}
u_{s}= & H_{s}\left(q_{s}\right)\left(g^{2} \alpha_{0}\left(\hat{q}_{m}-\xi_{1}\right)+\Lambda_{s}\left(\xi_{2}+g \alpha_{1}\left(\hat{q}_{m}-\xi_{1}\right)-\dot{q}_{s}\right)\right) \\
& +C_{s}\left(q_{s}, \dot{q}_{s}\right)\left(\xi_{2}+\Lambda_{s}\left(\xi_{1}-q_{s}\right)\right)+G_{s}\left(q_{s}\right)-K_{s}\left(\dot{q}_{s}-\xi_{2}+\Lambda_{s}\left(q_{s}-\xi_{1}\right)\right)(3.7)
\end{aligned}
$$

where $K_{s}, \Lambda_{s} \in \mathbb{R}^{n \times n}$ are symmetric positive definite matrices. Furthermore, $\xi_{1}, \xi_{2} \in$ $\mathbb{R}^{n}$ are estimates provided by the following "dirty-derivative" filter

$$
\begin{aligned}
& \dot{\xi}_{1}=\xi_{2}+g \alpha_{1}\left(\hat{q}_{m}-\xi_{1}\right), \\
& \dot{\xi}_{2}=g^{2} \alpha_{0}\left(\hat{q}_{m}-\xi_{1}\right),
\end{aligned}
$$


where $\alpha_{0}, \alpha_{1}$ are positive constants such that the roots of $p(s)=s^{2}+\alpha_{1} s+\alpha_{0}$ have negative real parts, and $g>0$.

The rationale behind the proposed control schemes is as follows. Our goal is to design a control law that makes both the master and the slave subsystem input-tostate stable; the overall stability of the teleoperator system then follows by application of the small gain arguments. In particular, both master control algorithms (3.5) and (3.6) make the master subsystem input-to-state stable. The difference between these control laws is that the algorithm (3.6) allows to assign an arbitrary ISS gain to the closed-loop master subsystem, which generally implies that stronger stability results can be achieved when using this control algorithm (for example, compare the statements of Theorem 2 and Theorem 3 below). On the other hand, the slave control algorithm (3.7), (3.8), (3.9) is essentially a passivity-based tracking control algorithm that also makes the interconnection of the slave and the environment input-to-state stable with respect to delayed master trajectory $\hat{q}_{m}(t)$. The filter (3.8), (3.9) has two main functions: first, it provides estimates for the first and second derivatives of the reference trajectory $\hat{q}_{m}(t)$, and second, it provides a smooth approximation of the reference trajectory in the case when (due to irregularity of the forward communication delay $\left.\tau_{f}(\cdot)\right)$ the reference trajectory on the slave side $\hat{q}_{m}(t):=q_{m}\left(t-\tau_{f}(t)\right)$ is nonsmooth.

\subsection{Force Reflection}

In this section, we define the force reflection signal $f_{r}$. In teleoperator systems, the force reflection signal may come from different sources. Two of the most common schemes are where the force reflecting term is proportional to tracking (position and/or velocity) error on the slave side (so called position-error based schemes), or 
to the actual contact force due to environment experienced by the slave manipulator (contact force reflection). In this proposal, we consider a general form of the force reflection term described as follows. First, let us define the "output" of the slaveenvironment subsystem, according to the following formula

$$
y_{s}=\Lambda_{1} f_{e}+\Lambda_{2}\left(q_{s}-\xi_{1}\right)+\Lambda_{3}\left(\dot{q}_{s}-\xi_{2}\right),
$$

where $\Lambda_{1}, \Lambda_{2}, \Lambda_{3}$ are symmetric $\left(\Lambda_{i}=\Lambda_{i}^{T}, i=1,2,3\right)$ nonnegative definite matrices satisfying $\Lambda_{1}+\Lambda_{2}+\Lambda_{3}=\mathbb{I}$. Thus, the "output" (3.10) is a weighted combination of the contact force due to environment $f_{e}$, slave position error $q_{s}-\xi_{1}$, and slave velocity error $\dot{q}_{s}-\xi_{2}$. Now, the force reflecting term $f_{r}$ is defined according to the formula

$$
f_{r}(t)=K_{f} y_{s}(t)
$$

where $K_{f} \geq 0$ is the force reflection gain.

Remark 4. The choice of $\Lambda_{1}, \Lambda_{2}, \Lambda_{3}$ in equation (3.10) depends on the source of the force reflection signal. For example, if the force reflection on the master side is based on direct measurement of the contact force due to environment, the appropriate choice would be $\Lambda_{1}=\mathbb{I}, \Lambda_{2}=\Lambda_{3}=\mathbb{O}$, while for the position error based schemes, where the force reflection on the master side is proportional to the slave's position error, we have $\Lambda_{2}=\mathbb{I}, \Lambda_{1}=\Lambda_{3}=\mathbb{O}$. Generally, using the appropriate choice of matrices $\Lambda_{1}, \Lambda_{2}, \Lambda_{3}$, the force reflection signal can be made proportional to either the actual contact force due to environment, position error, velocity error, or an arbitrary linear combination of all the above signals. 


\subsection{Environmental Dynamics}

In this proposal, we assume that the environment is described as a dynamical system of the following form

$$
\begin{aligned}
& \dot{x}_{e}=F_{e n v}\left(x_{e}, q_{s}, \dot{q}_{s}, f_{e x t}, t\right), \\
& f_{e}=G_{e n v}\left(x_{e}, q_{s}, \dot{q}_{s}, f_{e x t}, t\right),
\end{aligned}
$$

where $x_{e} \in \mathbb{R}^{q}$ is a state of the environment, $f_{e} \in \mathbb{R}^{n}$ the contact force due to environment applied to the slave manipulator, $q_{s}, \dot{q}_{s}$ are the position and velocity of the slave manipulator, and $f_{e x t}$ is an arbitrary measurable essentially bounded function that represents an equivalent of all external forces imposed on the environment; $q_{s}$, $\dot{q}_{s}, f_{e x t}$ all play the role of the inputs of the environmental dynamics. The righthand side of (3.12) depends on time $t$, i.e., the environment is possibly time-varying. We assume that $F_{e n v}(\cdot)$ is locally Lipschitz in $x_{e}, q_{s}, \dot{q}_{s}, \dot{f}_{\text {ext }}$, measurable in $t$, and essentially bounded on any compact set of $x_{e}, q_{s}, \dot{q}_{s}, f_{e x t}$ uniformly for all $t \in \mathbb{R}$. Additionally, it is assumed that $G_{e n v}(\cdot)$ is continuous in $x_{e}, q_{s}, \dot{q}_{s}, f_{e x t}$ uniformly for all $t \in \mathbb{R}$ and satisfies $G_{\text {env }}(0,0,0,0, t)=0$ for all $t \in \mathbb{R}$.

Our general assumption is that the environmental dynamics (3.12) are inputto-state stable. Under the above assumptions imposed on $G_{e n v}(\cdot)$, this also implies that the system (3.12) is input-to-output stable. For simplicity, it is additionally assumed that the corresponding IOS gains are linear. More precisely, the following assumption is imposed on the environmental dynamics (3.12).

Assumption 3. The environmental dynamics (3.12) is input-to-state stable. Moreover, there exist $\beta_{e} \in \mathcal{K}_{\infty}, \gamma_{f} \geq 0, \gamma_{e} \geq 0$ such that the contact force due to the environment, $f_{e}(t)$, satisfies the following properties:

i) uniform boundedness: for any $t_{0} \in \mathbb{R}$

$$
\sup _{t \geq t_{0}}\left|f_{e}(t)\right| \leq \max \left\{\beta_{e}\left(x_{e}\left(t_{0}\right)\right), \gamma_{e}\left(\sup _{t \geq t_{0}}\left|\begin{array}{l}
q_{s}(t) \\
\dot{q}_{\varepsilon}(t)
\end{array}\right|\right), \gamma_{f}\left(\sup _{t \geq t_{0}}\left|f_{e x t}(t)\right|\right)\right\} ;
$$


ii) convergence:

$$
\limsup _{t \rightarrow+\infty}\left|f_{e}(t)\right| \leq \max \left\{\gamma_{e}\left(\limsup _{t \rightarrow+\infty} \mid \begin{array}{l}
q_{s}(t) \\
\dot{q}_{s}(t)
\end{array}\right), \gamma_{f}\left(\limsup _{t \rightarrow+\infty}\left|f_{e x t}(t)\right|\right)\right\}
$$

\subsection{Stability}

In this section, general stability results for the force-reflecting teleoperator system are established. We address a general situation where the communication between the master and the slave manipulators is performed over an unreliable communication channel. In fact, in many telerobotic applications, it may be desirable to always transmit the most recent available information about the master's position to the slave side. In this case, if some packets of information are lost during the transmission, they are usually not sent again. In general, this results in discontinuity of the communication delay function, which may imply the discontinuity of the reference trajectory for the slave manipulator. Although in this case perfect tracking cannot be achieved because of such a discontinuity of the reference trajectory, however, as shown below, the proposed control scheme guarantees the overall stability which is uniform with respect to time delay in the communication channel. To formulate our results, let us introduce a set of state variables as follows

$$
\mathbf{x}_{t}:=\left(q_{m}^{T}, \dot{q}_{m}^{T}, \xi_{1}^{T}, \xi_{2}^{T}, \tilde{q}_{s}^{T}, \tilde{\dot{q}}_{s}^{T}, x_{e}^{T}\right)_{d}^{T}
$$

where $\tilde{q}_{s}:=q_{s}-\xi_{1}$, and $\tilde{\dot{q}}_{s}:=\dot{q}_{s}-\xi_{2}$, and where we use the notation

$x_{d}(t):=\left\{x(s), s \in\left[t-t_{d}(t), t\right]\right\}$ introduced in Chapter 2 , with $t_{d}: \mathbb{R} \rightarrow \mathbb{R}_{+}$defined below. The input of the teleoperator system is defined as follows

$$
u:=\left(f_{h}^{T}, f_{e x t}^{T}, \hat{\delta}_{q}^{T}, \hat{\delta}_{f}^{T}\right)^{T}
$$

where $\hat{\delta}_{q}, \hat{\delta}_{f}$ are errors due to quantization/measurement noise in the forward (3.3) and backward (3.4) communication channels, respectively. 
The following theorems describe stability properties of the closed-loop bilateral teleoperator system. First, let us formulate a stability result for the system with master control algorithm given by (3.5).

Theorem 2. Consider the bilateral teleoperator system (3.1), (3.2), (3.3), (3.4), (3.11), (3.12) with control law $(3.5),(3.7)-(3.9)$. Suppose the delays $\tau_{f}(\cdot), \tau_{b}(\cdot)$ in both the forward and the backward communication channels (3.3), (3.4) satisfy Assumption 1. Suppose also that the environmental dynamics (3.12) satisfy Assumption 3. Then there exist $\kappa_{s}>0, K_{f}^{*}>0$ such that $\lambda_{\min }\left(K_{s}\right) \geq \kappa_{s}, K_{f} \in\left[0, K_{f}^{*}\right)$ imply the closed-loop teleoperator system is input-to-state stable at $t=0$ with

$$
t_{d}=\max \left\{\tau_{f}(0)+\tau_{b}\left(-\tau_{f}(0)\right), \tau_{b}(0)+\tau_{f}\left(-\tau_{b}(0)\right)\right\}
$$

For the master control algorithm given by (3.6), the following stronger result can be obtained.

Theorem 3. Consider the system (3.1), (3.2), (3.3), (3.4), (3.11), (3.12) with control law $(3.6)-(3.9)$. Suppose the delays $\tau_{f}(\cdot), \tau_{b}(\cdot)$ in both the forward and the backward communication channels (3.3), (3.4) satisfy Assumption 1. Suppose also that the environmental dynamics (3.12) satisfy Assumption 3. Then, given $K_{f}^{*} \in[0,+\infty)$, there exist $\kappa_{m}>0, \kappa_{s}>0$ such that $\lambda_{\min }\left(K_{m}\right) \geq \kappa_{m}, \lambda_{\min }\left(K_{s}\right) \geq \kappa_{s}$ imply that the closed-loop teleoperator system is input-to-state stable at $t=0$ with $t_{d}$ defined by (3.17), for all $K_{f} \in\left[0, K_{f}^{*}\right)$.

Remark 5. Both Theorem 2 and Theorem 3 state stability results for the force-reflecting teleoperator system with irregular communications. The difference between these two results is essentially the following: Theorem 2 states that there exists an admissible upper bound for the force reflecting gain such that the overall system is stable, while Theorem 3 implies that an admissible upper bound for the force reflecting gain can be assigned arbitrarily by an appropriate choice of the 
master's local feedback gain matrix $K_{m}$. The reason for a stronger result to be obtained in Theorem 3 is that a more detailed master control law (3.6) is used.

\subsection{Tracking}

Besides contact stability, good free space tracking performance is important for majority of teleoperation tasks. In this section, we address tracking properties of the bilateral teleoperator system described above. Generally, in order to show that the slave tracks the (delayed) master trajectory, one needs to impose certain smoothness assumptions on communication delay $\tau_{f}(\cdot)$ in the feedforward channel. Indeed, "significant" discontinuity of the communication delay $\tau_{f}(\cdot)$ generally results in discontinuous reference trajectory for the slave manipulator for which rigorous tracking results cannot be obtained. Thus, to deal with tracking problem, one should assume that the discontinuity of the signal $\hat{q}_{m}$ is limited in some sense. One possible approach is to assume that "nominal" communication process in the feedforward channel (3.3) can be described by a smooth communication delay function $\tau_{f}(\cdot)$, while all the discontinuities (that, in particular, may correspond to discretization, jitter, packet loss, etc) are considered as disturbances and, therefore, are included in $\hat{\delta}_{q}$. More precisely, let us introduce the following assumption.

Assumption 4. The forward communication delay $\tau_{f}: \mathbb{R} \rightarrow \mathbb{R}_{+}$is a continuously differentiable function of time $t$ with the following properties:

i) $-\Upsilon_{1} \leq \frac{d \tau_{f}(t)}{d t} \leq 1$ for some $\Upsilon_{1} \geq 0$;

ii) $\left|\frac{d \tau_{f}}{d t}\left(t_{2}\right)-\frac{d \tau_{f}}{d t}\left(t_{1}\right)\right| \leq \Upsilon_{2}\left|t_{2}-t_{1}\right|$ for some $\Upsilon_{2} \geq 0$ and for all $t_{1}, t_{2} \in \mathbb{R}$;

iii) $t-\tau_{f}(t) \rightarrow+\infty$ as $t \rightarrow+\infty$.

Informally, we will say that the communication process in the forward channel is "approximately smooth" if $\hat{q}_{m}$ can be represented in the form (3.3) where $\tau_{f}(\cdot)$ 
satisfies Assumption 4, and $\hat{\delta}_{q}$ is sufficiently small. Note that, for sufficiently fine quantization, an approximately smooth communication can be achieved in practice by combining a reliable communication protocol and a buffering mechanism [38].

Below, we study the stability and tracking properties of the controlled telerobotic system under the assumption that the communication protocol in the forward communication channel (from the master to the slave) guarantees that $\tau_{f}(\cdot)$ satisfies Assumption 4. In this case, let us introduce some additional notation. Denote

$$
\hat{q}_{m}^{a}(t)=q_{m}\left(t-\tau_{f}(t)\right), \quad \dot{\hat{q}}_{m}^{a}(t):=\frac{d}{d t} \hat{q}_{m}^{a}(t)
$$

Roughly speaking, $\hat{q}_{m}^{a}, \hat{q}_{m}^{a}$ represent a "smooth component" of the delayed master position and its time derivative, respectively. Further, for the purposes of this section, it is convenient to decompose the input of the telerobotic system into two separate "channels", as follows

$$
u^{\{1\}}:=\left(f_{h}^{T}, f_{e x t}^{T}, \hat{\delta}_{f}^{T}\right)^{T}, \quad u^{\{2\}}:=\hat{\delta}_{q}
$$

Also, let us define an output of the closed-loop teleoperator system, according to the following formula

$$
y:=\left(\tilde{\xi}_{1}^{T}, \tilde{\xi}_{2}^{T}, \tilde{q}_{s}^{T}, \tilde{\dot{q}}_{s}^{T}\right)^{T}
$$

where $\tilde{\xi}_{1}=\hat{q}_{m}^{a}-\xi_{1}, \tilde{\xi}_{2}=\dot{\hat{q}}_{m}^{a}-\xi_{2}$. The output (3.20) essentially consists of the filter tracking error $\left(\tilde{\xi}_{1}^{T}, \tilde{\xi}_{2}^{T}\right)^{T}$ and the slave tracking error $\left(\tilde{q}_{s}^{T}, \tilde{q}_{s}^{T}\right)^{T}$. Below, the tracking properties of the closed-loop system will be described in terms of the IOS gain from input $u^{\{1\}}$ to output $y$. More precisely, the following result is valid.

Theorem 4. Consider the bilateral teleoperator system $(3.1),(3.2),(3.3),(3.4)$, (3.11), (3.12) with control law (3.5), (3.7) - (3.9). Suppose the communication delay $\tau_{f}(\cdot)$ in the forward channel (3.3) satisfies Assumption 4, while the communication delay $\tau_{b}(\cdot)$ in the backward channel (3.4) satisfies Assumption 1. Suppose also that 
the environmental dynamics (3.12) satisfy Assumption 3. Then there exist $\kappa_{s}>0$, $K_{f}^{*}>0$ such that $\lambda_{\min }\left(K_{s}\right) \geq \kappa_{s}, K_{f} \in\left[0, K_{f}^{*}\right)$ imply the closed-loop teleoperator system is input-to-state stable at $t=0$ with $t_{d}$ defined by (3.17). Moreover, the IOS gain from input $u^{\{1\}}$ to output $y$ can be made arbitrarily small by an appropriate choice of the parameters $K_{s}, \Lambda_{s}$ and $g$.

In the case of master control algorithm (3.6), the following result is valid.

Theorem 5. Consider the system (3.1), (3.2), (3.3), (3.4), (3.11), (3.12) with control law $(3.6)-(3.9)$. Suppose the communication delay $\tau_{f}(\cdot)$ in the forward channel (3.3) satisfies Assumption 4, while the communication delay $\tau_{b}(\cdot)$ in the backward channel (3.4) satisfies Assumption 1. Suppose also that the environmental dynamics (3.12) satisfy Assumption 3. Then, given $K_{f}^{*} \in[0,+\infty)$, there exist $\kappa_{m}>0, \kappa_{s}>0$ such that $\lambda_{\min }\left(K_{m}\right) \geq \kappa_{m}, \lambda_{\min }\left(K_{s}\right) \geq \kappa_{s}$ imply that the closedloop teleoperator system is input-to-state stable at $t=0$ with $t_{d}$ defined by (3.17), for all $K_{f} \in\left[0, K_{f}^{*}\right)$. Moreover, the IOS gain from input $u^{\{1\}}$ to output $y$ can be made arbitrarily small by an appropriate choice of the parameters $K_{s}, \Lambda_{s}$ and $g$.

Remark 6. The results of Theorems 4,5 can be given the following interpretation. In addition to the input-to-state stability of the overall teleoperator system, it can be shown that the slave tracks the delayed master trajectory with error that depends on magnitude of the forces applied as well as magnitude of the quantization/measurement error terms $\hat{\delta}_{q}, \hat{\delta}_{f}$. If the term $\hat{\delta}_{q}$ is negligible, which corresponds to the case of "approximately smooth" communication in the forward communication channel, then an arbitrarily precise tracking can be achieved by appropriate choice of the feedback matrices and the filter constant $g$. More precisely, if $\hat{\delta}_{q} \approx 0$, then for a given maximal magnitude of the external forces, and a maximal magnitude of the quantization/measurement error in the backward channel, the following two properties of the closed-loop teleoperator system can be guaranteed uniformly 
for all $\tau_{f}(\cdot), \tau_{b}(\cdot)$ by an appropriate choice of the feedback matrices $K_{m}, K_{s}$ and the filter constant $g:$ i) the dirty-derivative filter gives an arbitrary precise estimate of the delayed trajectory of the master, and ii) the slave manipulator tracks the trajectory provided by the dirty-derivative filter with error less than or equal to a prescribed bound.

\subsection{Proofs}

\section{Proof of Theorem 2 and 3}

Below, proofs of both Theorem 2 and Theorem 3 will be given through a sequence of propositions. Both these proofs follow the same line of reasoning. First, we show the proposed control laws make both the master and the slave subsystems are input-tostate stable. Then, proofs are finalized by application of the small gain arguments (Theorem 1). Let us first formulate two results that describe stability properties of the master subsystem equipped with control laws (3.5) and (3.6), respectively. Let us define a state and input of the master subsystem, as follows,

$$
\begin{gathered}
x_{M}:=\left(q_{m}^{T}, \dot{q}_{m}^{T}\right)^{T}, \\
u_{M}:=f_{h}-\hat{f}_{r} .
\end{gathered}
$$

First, we address the ISS properties of the subsystem "master manipulator (3.1) + control law (3.5)". The following result is proven in [62].

Proposition 1. The closed-loop master subsystem (3.1), (3.5) is input-to-state stable.

Next, consider the master subsystem with control law (3.6). Substituting (3.6) into the equations of the master manipulator (3.1), and using the variable $e_{m}=$ $\dot{q}_{m}+\Lambda_{m} q_{m}$, we get the following equations of the closed-loop master subsystem in 
terms of state variables $q_{m}, e_{m}$ :

$$
\begin{aligned}
& \dot{q}_{m}=-\Lambda_{m} q_{m}+e_{m}, \\
& \dot{e}_{m}=H_{m}^{-1}\left(q_{m}\right)\left(-C_{m}\left(q_{m}, e_{m}-\Lambda_{m} q_{m}\right) e_{m}-K_{m} e_{m}+f_{h}-\hat{f}_{r}\right) .
\end{aligned}
$$

Note that the state (3.21) can be obtained from $\left(q_{m}^{T}, e_{m}^{T}\right)^{T}$ by the following nonsingular coordinate transformation

$$
x_{M}:=\left(\begin{array}{c}
q_{m} \\
\dot{q}_{m}
\end{array}\right)=\left(\begin{array}{cc}
1 & 0 \\
\Lambda_{m} & 1
\end{array}\right)^{-1}\left(\begin{array}{c}
q_{m} \\
e_{m}
\end{array}\right) .
$$

Let us also define the following two outputs for the system (3.23), (3.24):

$$
y_{M}^{\{1\}}:=\left(q_{m}^{T}, \dot{q}_{m}^{T}\right)^{T}=x_{M}, \quad y_{M}^{\{2\}}:=\ddot{q}_{m}
$$

The following proposition describes the IOS/ISS properties of the closed loop master subsystem (3.23), (3.24).

Proposition 2. Given $\gamma_{m}^{\{1\}}>0$, there exists $\kappa_{m} \geq 0$ such that if the matrix $K_{m}$ satisfies $\lambda_{\min }\left(K_{m}\right) \geq \kappa_{m}$, then the closed-loop master subsystem (3.23), (3.24) with state (3.21) and input (3.22) is input-to-state stable, and the corresponding ISS gains are less than or equal to $\gamma_{m}^{\{1\}}$. Moreover, there exists $\gamma_{m}^{\{2\}} \in \mathcal{K}$ independent of $\gamma_{m}^{\{1\}}$ and satisfying

$$
\limsup _{s \rightarrow 0+} \frac{\gamma_{m}^{\{2\}}(s)}{s}<+\infty
$$

such that the IOS gain from $u_{M}$ to $y_{M}^{\{2\}}$ are less than or equal to $\gamma_{m}^{\{2\}}$.

Proof Consider the ISS Lyapunov function candidate

$$
V_{m}:=\frac{1}{2} e_{m}^{T} H_{m}\left(q_{m}\right) e_{m}+\frac{1}{2} q_{m}^{T} q_{m} .
$$

Since the matrix $H_{m}\left(q_{m}\right)$ is positive definite for all $q_{m} \in \mathbb{Q}_{m}$ [61], it can be easily checked using (3.25) that there exist $\alpha_{1 m}>0, \alpha_{2 m}>0, \alpha_{1 m} \leq \alpha_{2 m}$ such that

$$
\alpha_{1 m}\left(\left|\dot{q}_{m}\right|^{2}+\left|q_{m}\right|^{2}\right) \leq V_{m} \leq \alpha_{2 m}\left(\left|\dot{q}_{m}\right|^{2}+\left|q_{m}\right|^{2}\right)
$$


Calculating the time derivative of $V_{m}$ along the trajectories of the closed-loop master subsystem, taking into account the skew-symmetry of $\dot{H}_{m}-2 C_{m}$ (see [61]), and completing the squares, one can get that the following estimate

$$
\dot{V}_{m} \leq-\frac{1}{2}\left(e_{m}^{T} K_{m} e_{m}+q_{m}^{T} \Lambda_{m} q_{m}\right)+\frac{3}{2 \lambda_{\min }\left(K_{m}\right)}\left(\left|f_{h}-\hat{f}_{r}\right|^{2}\right)
$$

holds if $\lambda_{\min }\left(K_{m}\right)>0$ is sufficiently large. This implies [63] that the system is ISS, and the corresponding ISS gains can be assigned arbitrarily low by increasing $\lambda_{\text {min }}\left(K_{m}\right)>0$. Finally, the IOS property with respect to output $y_{M}^{\{2\}}$ as well as the inequality (3.27) can be checked by straightforward calculations which are omitted here due to space reasons.

Next, consider the closed-loop slave subsystem (3.2), (3.7). Denote

$$
\tilde{q}_{s}:=q_{s}-\xi_{1}, \quad \tilde{q}_{s}:=\dot{q}_{s}-\xi_{2}, \quad e_{s}:=\tilde{q}_{s}+\Lambda_{s} \tilde{q}_{s}
$$

In these notations, the equations (3.2), (3.7) can be rewritten as follows

$$
\begin{aligned}
& \dot{e}_{s}=H_{s}^{-1}\left(q_{s}\right)\left(-C_{s}\left(q_{s}, \dot{q}_{s}\right) e_{s}-K_{s} e_{s}-f_{e}\right) \\
& \dot{\tilde{q}}_{s}=-\Lambda_{s} \tilde{q}_{s}+e_{s}-g \alpha_{1}\left(\hat{q}_{m}-\xi_{1}\right) .
\end{aligned}
$$

Keeping in mind that

$$
\left(\begin{array}{c}
\tilde{\tilde{q}}_{s} \\
\tilde{q}_{s}
\end{array}\right)=\left(\begin{array}{cc}
1 & \Lambda_{s} \\
0 & 1
\end{array}\right)^{-1}\left(\begin{array}{l}
e_{s} \\
\tilde{q}_{s}
\end{array}\right),
$$

one can define a state of the closed-loop system as follows

$$
x_{S}:=\left(\tilde{q}_{s}^{T}, \tilde{q}_{s}^{T}\right)^{T}
$$

Proposition 3. The closed-loop slave subsystem (3.31), (3.32) with state (3.34) and inputs $f_{e}, \tilde{\varsigma}_{1}:=g\left(\hat{q}_{m}-\xi_{1}\right)$ is input-to-state stable. Moreover, given $\gamma_{s}>0$, there exists $\kappa_{s} \geq 0$ such that $\lambda_{\min }\left(K_{s}\right) \geq \kappa_{s}$ implies the ISS gain with respect to input $f_{e}$ is less than or equal to $\gamma_{s}$. 
Proof. Consider the ISS Lyapunov function candidate

$$
V=\frac{1}{2} e_{s}^{T} H_{s}\left(q_{s}\right) e_{s}+\frac{1}{2} \tilde{q}_{s}^{T} \tilde{q}_{s}
$$

Taking into account (3.33), we see that

$$
\alpha_{\min }\left(\left|\tilde{\dot{q}}_{s}\right|^{2}+\left|\tilde{q}_{s}\right|^{2}\right) \leq V \leq \alpha_{\max }\left(\left|\tilde{\tilde{q}}_{s}\right|^{2}+\left|\tilde{q}_{s}\right|^{2}\right)
$$

for some $\alpha_{\min }, \alpha_{\max }>0$. Calculating the time derivative of $V$ along the trajectories of the system (3.31), (3.32), and completing the squares, we get

$$
\begin{gathered}
\dot{V} \leq-e_{s}^{T} K_{s} e_{s}+e_{s}^{T} f_{e}+\tilde{q}^{T}\left(-\Lambda_{s} \tilde{q}+e_{s}+\alpha_{1} \tilde{\varsigma}_{1}\right) \\
\leq-\frac{3}{4} e_{s}^{T} K_{s} e_{s}-\frac{1}{2} \hat{q}_{s}^{T} \Lambda_{s} \tilde{q}_{s}+\frac{1}{\lambda_{\min }\left(K_{s}\right)}\left|f_{e}\right|^{2}+\frac{1}{\lambda_{\min }\left(\Lambda_{s}\right)}\left|e_{s}\right|^{2}+\frac{1}{\lambda_{\min }\left(\Lambda_{s}\right)} \alpha_{1}^{2}\left|\tilde{\zeta}_{1}\right|^{2} .
\end{gathered}
$$

Taking into account (3.33), and applying the results presented in [63], one get that (3.37) implies the input-to-state stability of the closed-loop slave subsystem (3.31), (3.32). Further, it is easy to see that the ISS gain with respect to the input $f_{e}$ can be assigned arbitrarily low by increasing $\lambda_{\min }\left(K_{s}\right)>0$.

Next, consider the interconnection of the closed-loop slave subsystem (3.31), (3.32) with the environment described by equation (3.12). This interconnection has inputs $\xi=\left(\xi_{1}^{T}, \xi_{2}^{T}\right)^{T}, f_{\text {ext }}$, and $\hat{q}_{m}$, and a state of the interconnection can be defined as $\mathbf{x}_{S+E}:=\left(\tilde{q}_{s}^{T}, \tilde{\dot{q}}_{s}^{T}, x_{e}^{T}\right)^{T}$. According to Proposition 3 and Assumption 3 , both the subsystems are ISS, and the corresponding small gain stability condition has a form

$$
\gamma_{e} \gamma_{s}<1
$$

According to Proposition $3, \gamma_{s}>0$ can be assigned arbitrarily by an appropriate choice of $K_{s}$, therefore condition (3.38) can always be satisfied.

Now, consider the filter (3.8), (3.9). Define a new set of coordinates $\varsigma=$ $\left(\varsigma_{1}^{T}, \varsigma_{2}^{T}\right)^{T}:=\left(g \xi_{1}^{T}, \xi_{2}^{T}\right)^{T}$. In the new coordinates the equations (3.8), (3.9) become

$$
\dot{\varsigma}=g A \varsigma+g^{2} B \hat{q}_{m}
$$


where

$$
A:=\left[\begin{array}{cc}
-\alpha_{1} \mathbb{I}_{n \times n} & \mathbb{I}_{n \times n} \\
-\alpha_{0} \mathbb{I}_{n \times n} & \mathbb{O}_{n \times n}
\end{array}\right], B:=\left[\begin{array}{c}
\alpha_{1} \mathbb{I}_{n \times n} \\
\alpha_{0} \mathbb{I}_{n \times n}
\end{array}\right] .
$$

Proposition 4. The system (3.39) is ISS with ISS-Lyapunov function $V=$ $\varsigma^{T} P \varsigma$, where $P=P^{T}>0$ is the (unique) solution of the matrix Lyapunov equation

$$
A^{T} P+P A=-\mathbb{I}
$$

The proof of the above proposition is straightforward.

Now, the proofs of Theorem 2 and Theorem 3 can be finalized as follows. First, note that the interconnection of the filter (3.8), (3.9) and the "controlled slave + environment" (3.31), (3.32), (3.12) systems has a cascade structure and, therefore, is ISS as a cascade interconnection of two ISS subsystems. Let $y_{s}$ defined by (3.10) be the output of the "filter + controlled slave + environment" interconnection, and let $\gamma_{F S E}>0$ be the corresponding IOS gain (from input $\hat{q}_{m}$ to output $y_{s}$ ). According to Proposition 1, the master subsystem with control law (3.5) is ISS. Let, therefore, $\gamma_{m}>0$ be the corresponding ISS gain. Applying the small gain result (Theorem 1), we see that the small gain stability condition has a form

$$
\gamma_{m} \cdot K_{f} \cdot \gamma_{F S E}<1
$$

which implies that the overall system is stable for all $K_{f} \in\left[0, K_{f}^{*}\right)$, where $K_{f}^{*}=$ $\left(\gamma_{m} \cdot \gamma_{F S E}\right)^{-1}$. This proves Theorem 2. To prove Theorem 3, note that in the case of master control law (3.6), according to Proposition 2, the master gain $\gamma_{m}>0$ can be assigned arbitrarily. Therefore, given $K_{f}^{*}>0, \gamma_{F S E}>0$, the overall teleoperator system is stable for all $K_{f} \in\left[0, K_{f}^{*}\right)$ whenever $\gamma_{m} \leq\left(K_{f}^{*} \cdot \gamma_{F S E}\right)^{-1}$. This completes the proof of Theorem 3 . 


\section{Proof of Theorem 4 and 5}

First, note that the assumptions of Theorems 4, 5 are strictly stronger that the ones of Theorems 2, 3; thus, the stability parts of both Theorem 4 and Theorem 5 follow directly from the results of Theorem 2 and 3, respectively. It remains to prove that the IOS gain from input $u^{\{1\}}$ to output $y$ can be made arbitrarily small by an appropriate choice of the parameters $K_{s}, \Lambda_{s}$ and $g$. By assumption, the forward communication channel is described by equation (3.3), where $\tau_{f}(t)$ satisfies Assumption 4, i) - iii). Furthermore, by Assumption 4, ii), we see that the first time

derivative of $\tau_{f}(\cdot)$ is an uniformly Lipschitz function, therefore (see for example [64]), $d^{2} \tau_{f} / d t^{2}$ is well-defined for almost all $t \geq 0$, and satisfies

$$
\sup _{t \geq 0}\left|d^{2} \tau_{f}(t) / d t^{2}\right| \leq \Upsilon_{2}
$$

Let us denote $\hat{\dot{q}}_{m}^{a}(t):=\dot{q}_{m}\left(t-\tau_{f}(t)\right), \quad \hat{\tilde{q}}_{m}^{a}(t):=\ddot{q}_{m}\left(t-\tau_{f}(t)\right)$. We have

$$
\dot{\hat{q}}_{m}^{a}(t):=\frac{d}{d t} \hat{q}_{m}^{a}=\hat{\dot{q}}_{m}^{a}(t)\left(1-\frac{d \tau_{f}(t)}{d t}\right)
$$

and

$$
\ddot{\hat{q}}_{m}^{a}(t):=\frac{d^{2}}{d t^{2}} \hat{q}_{m}^{a}=\hat{\tilde{q}}_{m}^{a}(t) \cdot\left[1-\frac{d \tau_{f}(t)}{d t}\right]^{2}-\hat{\dot{q}}_{m}^{a}(t) \frac{d^{2} \tau_{f}}{d t^{2}},
$$

where the last equality is valid for almost all $t \geq 0$. Using Assumption 4 , i), ii), we get

$$
\left|\dot{\hat{q}}_{m}^{a}(t)\right| \leq \bar{\Upsilon}_{1}\left|\hat{\dot{q}}_{m}^{\alpha}(t)\right|
$$

and

$$
\left|\ddot{\tilde{q}}_{m}^{a}(t)\right| \leq 2 \bar{\Upsilon}_{2} \max \left\{\left|\hat{\hat{q}}_{m}^{a}(t)\right|,\left|\hat{\hat{q}}_{m}^{a}(t)\right|\right\},
$$

where $\bar{\Upsilon}_{1}:=\Upsilon_{1}+1, \bar{\Upsilon}_{2}:=\left[\left(\Upsilon_{1}+1\right)^{2}+\Upsilon_{2}\right]$.

Now, consider the equations of the filter (3.8), (3.9). Denote $\tilde{\xi}_{1}=\hat{q}_{m}^{a}-\xi_{1}$, $\tilde{\xi}_{2}=\dot{\hat{q}}_{m}^{a}-\xi_{2}$, and, analogously to the proof of Theorems 2,3 , let us introduce a 
new set of coordinates $\tilde{\varsigma}=\left(\tilde{\varsigma}_{1}^{T}, \tilde{\varsigma}_{2}^{T}\right)^{T}:=\left(g \tilde{\xi}_{1}^{T}, \tilde{\xi}_{2}^{T}\right)^{T}$. In the new coordinates, the equations (3.8), (3.9) become

$$
\dot{\tilde{\zeta}}=g A \tilde{\varsigma}+B \ddot{\hat{q}}_{m}^{a}+g^{2} B \hat{\delta}_{q}
$$

where matrices $A, B$ are defined by (3.40). Using the ISS Lyapunov function candidate $V=\tilde{\varsigma}^{T} P \tilde{\zeta}$, where $P=P^{T}>0$ is the solution of (3.41), it is trivial to check that the system (3.44) with inputs $\ddot{\hat{q}}_{m}, \hat{\delta}_{q}$ is input-to-state stable, and the ISS gain $\gamma_{\left[\hat{\tilde{q}}_{m \rightarrow \xi}\right]}>0$ corresponding to input $\ddot{\hat{q}}_{m}$ can be assigned arbitrarily small by choosing sufficiently large $g>0$.

As a next step, recall that the "slave+environment" interconnection is ISS (and IOS) if (3.38) holds. Therefore, the "filter+slave+environment" subsystem (3.12), (3.14), (3.31), (3.32), (3.42)-(3.44) with state $\left(\tilde{q}_{s}^{T}, \tilde{q}_{s}^{T}, \tilde{\varsigma}^{T}\right)^{T}$, inputs $\hat{q}_{m}^{a}, \hat{\dot{q}}_{m}^{a}, \hat{\ddot{q}}_{m}^{a}, \hat{\delta}_{q}$, and output $y_{s}$ is IOS (and ISS) as a cascade connection of two IOS (ISS) subsystems. Moreover, Proposition 3 implies that the gain with respect to input $f_{e}$ can be assigned arbitrarily small. Taking into account Assumption 3, one sees that the ISS gain with respect to input $f_{\text {ext }}$ can be made arbitrarily small by choosing $\lambda_{\min }\left(K_{s}\right)>0$ sufficiently large. Also, combining (3.43) with the ISS properties of the filter (3.44), we see that the IOS gain $\gamma_{\left[\hat{q}_{m \rightarrow} \rightarrow y_{s}\right]}>0$ from the "input" $\hat{\ddot{q}}_{m}^{a}$ to the output $y_{s}$ can also be assigned arbitrary small by choosing $g>0$ sufficiently large. The rest of the proof is similar to the corresponding parts of the proofs of Theorems 2 and 3 . •. 


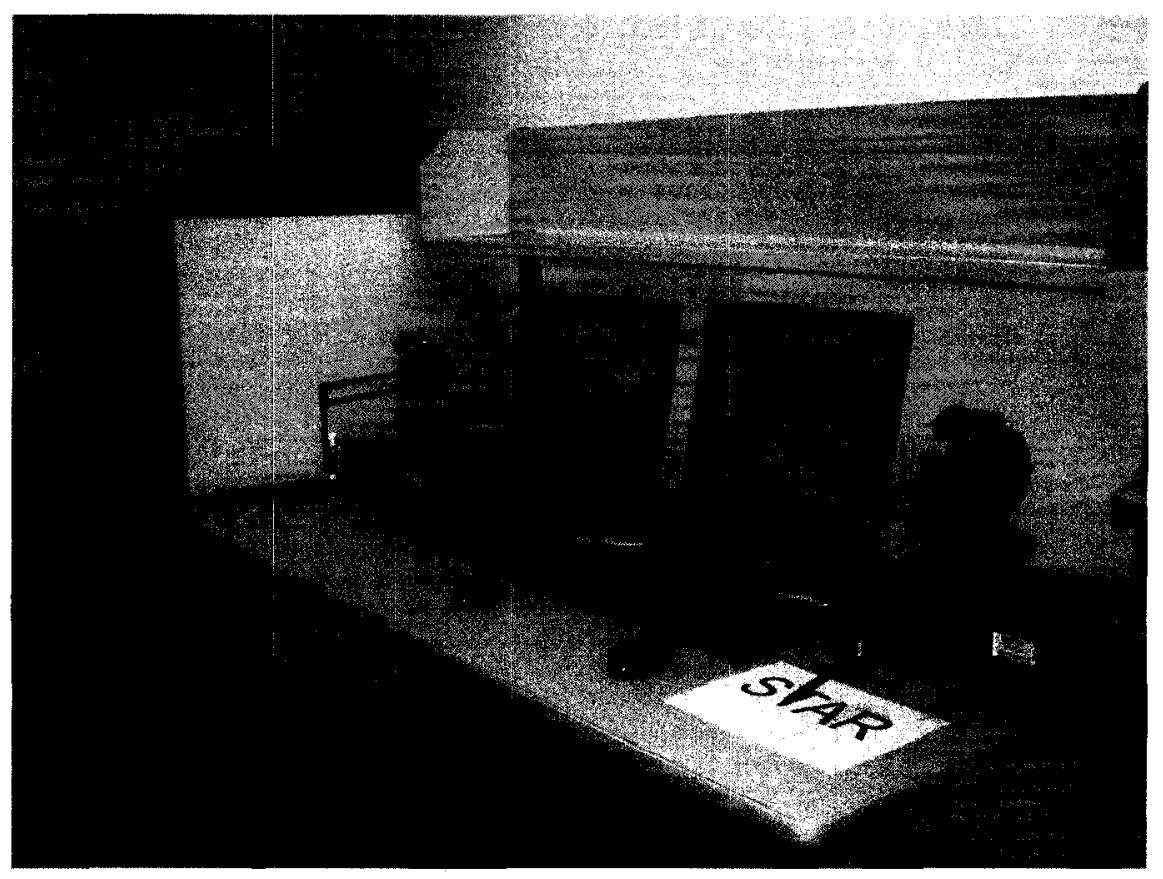

Figure 3.1: Experimental setup

\subsection{Experimental Results}

In this section, some results of experimental evaluation of the above proposed small gain based schemes for bilateral teleoperation over networks are presented. More precisely, we evaluate stability and performance of the position error based scheme described by equations (3.1), (3.2), (3.3), (3.4), (3.11), (3.12) with control law (3.5), (3.7) - (3.9); the corresponding stability result is given by Theorem 2. The telerobotic system used in our experiments is shown in Figure 3.1. It includes two Phantom Premium 1.5A robotic arms with 3 degrees of freedom positional sensing which are provided by SensAble Technologies, Inc.. The master manipulator is equipped with standard passive stylus and thimble gimbal, while the slave has a marker attached to its last link. As a preliminary step, modelling and parameter identification procedures of the Phantom Premium 1.5A devices were performed. A complete account of these procedures is given in Appendix A. As a result, the 
following model of the Phantom Premium 1.5A is obtained

$$
\left[\begin{array}{ccc}
M_{11} & 0 & 0 \\
0 & M_{22} & M_{23} \\
0 & M_{32} & M_{33}
\end{array}\right]\left[\begin{array}{c}
\ddot{q}_{1} \\
\ddot{q}_{2} \\
\ddot{q}_{3}
\end{array}\right]+\left[\begin{array}{ccc}
C_{11} & C_{12} & C_{13} \\
C_{21} & C_{22} & C_{23} \\
C_{31} & 0 & 0
\end{array}\right]\left[\begin{array}{c}
\dot{q}_{1} \\
\dot{q}_{2} \\
\dot{q}_{3}
\end{array}\right]+\left[\begin{array}{c}
K_{f v 1} \dot{q}_{1}+K_{f c 1} \operatorname{sign} \dot{q}_{1} \\
G_{2}+K_{f v 2} \dot{q}_{2}+K_{f c 2} \operatorname{sign} \dot{q}_{2} \\
G_{3}+K_{f v 3} \dot{q}_{3}+K_{f c 3} \operatorname{sign} \dot{q}_{3}
\end{array}\right]=\left[\begin{array}{c}
\tau_{1} \\
\tau_{2} \\
\tau_{3}
\end{array}\right],
$$

where $\tau_{i}$ are joint torques, $M_{i j}$ are the elements of inertia matrix, $C_{i j}$ are elements of the matrix of Coriolis and centrifugal forces (torques), $G_{i}$ are elements of the gravity vector, and $K_{f v}, K_{f c}$ are viscous and Coulomb friction coefficients, respectively. In the above equations,

$$
\begin{aligned}
M_{11}= & \pi_{1}+\pi_{2} \cos ^{2} q_{2}+\left(\pi_{3}+\pi_{5}\right) \sin ^{2} q_{3}+2 \pi_{6} \cos q_{2} \sin q_{3} \\
M_{22}= & \pi_{4}+\pi_{5}-2 \pi_{6} \sin \left(q_{2}-q_{3}\right) \\
M_{23}= & M_{32}=\pi_{5}-\pi_{6} \sin \left(q_{2}-q_{3}\right) \\
M_{33}= & \pi_{5} \\
C_{11}= & -\left(\pi_{2} \sin q_{2} \cos q_{2}+\pi_{6} \sin q_{2} \sin q_{3}\right) \dot{q}_{2} \\
& +\left(\left(\pi_{3}+\pi_{5}\right) \sin q_{3} \cos q_{3}+\pi_{6} \cos q_{2} \cos q_{3}\right) \dot{q}_{3} \\
C_{12}= & -\left(\pi_{2} \sin q_{2} \cos q_{2}+\pi_{6} \sin q_{2} \sin q_{3}\right) \dot{q}_{1} \\
C_{13}= & \left(\left(\pi_{3}+\pi_{5}\right) \sin q_{3} \cos q_{3}+\pi_{6} \cos q_{2} \cos q_{3}\right) \dot{q}_{1} \\
C_{21}= & \left(\pi_{2} \sin q_{2} \cos q_{2}+\pi_{6} \sin q_{2} \sin q_{3}\right) \dot{q}_{1} \\
C_{22}= & \pi_{6} \cos \left(q_{2}-q_{3}\right)\left(\dot{q}_{3}-\dot{q}_{2}\right) \\
C_{23}= & \pi_{6} \cos \left(q_{2}-q_{3}\right)\left(\dot{q}_{2}-\dot{q}_{3}\right) \\
C_{31}= & \Gamma_{311} \dot{q}_{1}=-\left(\pi_{3}+\pi_{5}\right) \sin q_{3} \cos q_{3} \dot{q}_{1}-\pi_{6} \cos q_{2} \cos q_{3} \dot{q}_{1} \\
G_{2}= & \pi_{7} \cos q_{2}, \quad G_{3}=\pi_{8} \sin q_{3}, K_{f v 1}=\pi_{9}, K_{f v 2}=\pi_{10}, K_{f v 3}=\pi_{11} \\
\quad & K_{f c 1}=\pi_{12}, K_{f c 2}=\pi_{13}, K_{f c 3}=\pi_{14} \\
&
\end{aligned}
$$

It is worth noting that in the software implementation used in our experiments, the position in joint space is measured in units of radians, while the joint space input (torque) is a numerical value between -32768 to 32767 . The parameter identification 
procedure gives the following values of the parameters $\pi_{1}, \ldots, \pi_{14}$ of the master manipulator: $\pi_{1}=50, \pi_{2}=60, \pi_{3}=48, \pi_{4}=80, \pi_{5}=9, \pi_{6}=8, \pi_{7}=235$, $\pi_{8}=270, \pi_{9}=25, \pi_{10}=22, \pi_{11}=110, \pi_{12}=82, \pi_{13}=160, \pi_{14}=90$. For the slave manipulator, the parameters are identified as follows: $\pi_{1}=32, \pi_{2}=34$, $\pi_{3}=20, \pi_{4}=74, \pi_{5}=1, \pi_{6}=2, \pi_{7}=-926, \pi_{8}=-685, \pi_{9}=15, \pi_{10}=15$, $\pi_{11}=90, \pi_{12}=80, \pi_{13}=112, \pi_{14}=55$. Throughout this section, the parameters of the control law (3.5), (3.7) - (3.9) are chosen as follows $\Lambda_{m}=\operatorname{diag}\{2,2,2\}$, $\Lambda_{s}=\operatorname{diag}\{5,5,5\}, K_{m}=\operatorname{diag}\{10,10,10\}, K_{s}=\operatorname{diag}\{300,300,300\}, g=10$, $\alpha_{1}=4, \alpha_{0}=4$.

In the experiments presented below, the end-effector of the master manipulator is initially located approximately at the origin of the task space coordinate frame; in particular, $x(0) \approx 0 \mathrm{~m}$. Starting from approximately $t=10$ seconds, the human operator quickly moves the end-effector of the master along the negative direction of the $\mathrm{X}$-axis, then returns it back to the origin and immediately releases. Figure 3.2 shows an example of the movement of the master manipulator which is intended by the human operator. When slave follows the master's trajectory, it hits an obstacle which is located approximately at $x=-0.25 \mathrm{~m}$. The obstacle is a rigid wall with very high stiffness. The quick movement together with a contact with a rigid environment create a strong destabilizing effect; the goal of the experiments presented is to evaluate the resulting response of the master-slave teleoperator system, in particular in the presence of communication delay and jitter. The form of the experiment described above is chosen because, as our preliminary experiments show, the instability only arises during free motion after the operator releases the master manipulator rather than during contact with the environment. In other words, no instability was observed in the experiments if the operator still held the master at the end. This observation is actually consistent with what found by other re- 
searchers $[65 ; 15]$ : a rigid grasp of the control handle is likely to stabilize the system while a loose grasp or the release of the master tends to make the system unstable.

Below, we present examples of experimental results for different values of force reflecting gain as well as different communication delay characteristics. According to the theoretical considerations presented above, it is expected that there exists an admissible upper bound for the force reflecting gain such that the overall system is stable for all communication delays, as long as the force reflecting gain satisfies this bound. It is also naturally expected that for small (negligible) communication delays, the stability is preserved for higher values of the force reflecting gain comparing to the case of significant communication delays. Both these hypotheses are confirmed by our experimental results. In particular, responses of the teleoperator system for the case of negligible communication delays are presented in Figures 3.43.7; these figures correspond to the values of the force reflecting gain equal to 500 , 1500,3000 , and 4000, respectively. As expected, the system is stable for low values of the force reflection gain, and unstable for high ones. Also, it can be seen from Figures 3.4-3.7, that the border of stability in terms of admissible FR gain in the case of negligible communication delay lies somewhere between 3000 (still stable, Figure 3.6) and 4000 (unstable, Figure 3.7).

On the other hand, we also address the case where significantly large irregular communication delays are present in both the forward (from master to slave) and the backward (from slave to master) communication channels. More precisely, we consider a situation where both the forward $\tau_{f}(\cdot)$ and the backward $\tau_{b}(\cdot)$ communication delays are modelled as random variables with normal (Gaussian) distribution characterized by its mean $T_{a v}$ and standard deviation $\sigma$ (the following standard notation $\tau(\cdot) \sim \mathbb{N}\left(T_{a v}, \sigma^{2}\right)$ is used). Example of such a communication delay function (with $T_{a v}=1 \mathrm{sec}$, and $\sigma=0.02$ ) is shown in Figure 3.3. It is worth noting that 
normally distributed communication delay functions are considered here just as an example, while other forms of communication delays are also possible, due to the fact that our stability results are proven under a very general assumption on communication delay (Assumption 1) which, in particular, does not specify exact form of the probability distribution function. Experimental results for $\tau_{f}(\cdot), \tau_{b}(\cdot) \sim \mathbb{N}\left(T_{a v}, \sigma^{2}\right)$, $T_{a v}=1 \mathrm{sec}, \sigma=0.02$, are shown in Figures 3.8-3.10; these figures correspond to FR gains equal to 500,1000 , and 1500, respectively. Although the qualitative picture is the same (i.e., the system is stable for low FR gains, and unstable for high ones), the border of stability in this case is significantly lower comparing to the case of negligible communication delays; namely, it lies somewhere between 1000 (stable, Figure 3.9) and 1500 (unstable, Figure 3.10). It is interesting to note that further increasing the communication delay does not lead to decreasing the admissible FR gain; in particular, the system with FR gain equal to 1000 remains stable for significantly higher values of communication delay. Example of the response for $\tau_{f}(\cdot), \tau_{b}(\cdot) \sim \mathbb{N}\left(T_{a v}, \sigma^{2}\right), T_{a v}=2.5 \mathrm{sec}, \sigma=0.05$, and $\mathrm{FR}$ gain equal to 1000 is shown in Figure 3.11; one can see that it is stable, and the performance of the response is visually very similar to the one with $T_{a v}=1 \mathrm{sec}, \sigma=0.02$.

Overall, the experiments performed confirm that, for the small-gain schemes proposed in this chapter, there exists an upper bound for the force reflecting gain such that the force-reflecting teleoperator system is stable for all FR gains that satisfy this bound, regardless of the communication delay characteristics. An important question is how to improve this bound without losing the overall stability. This problem is addressed in the next chapter. 


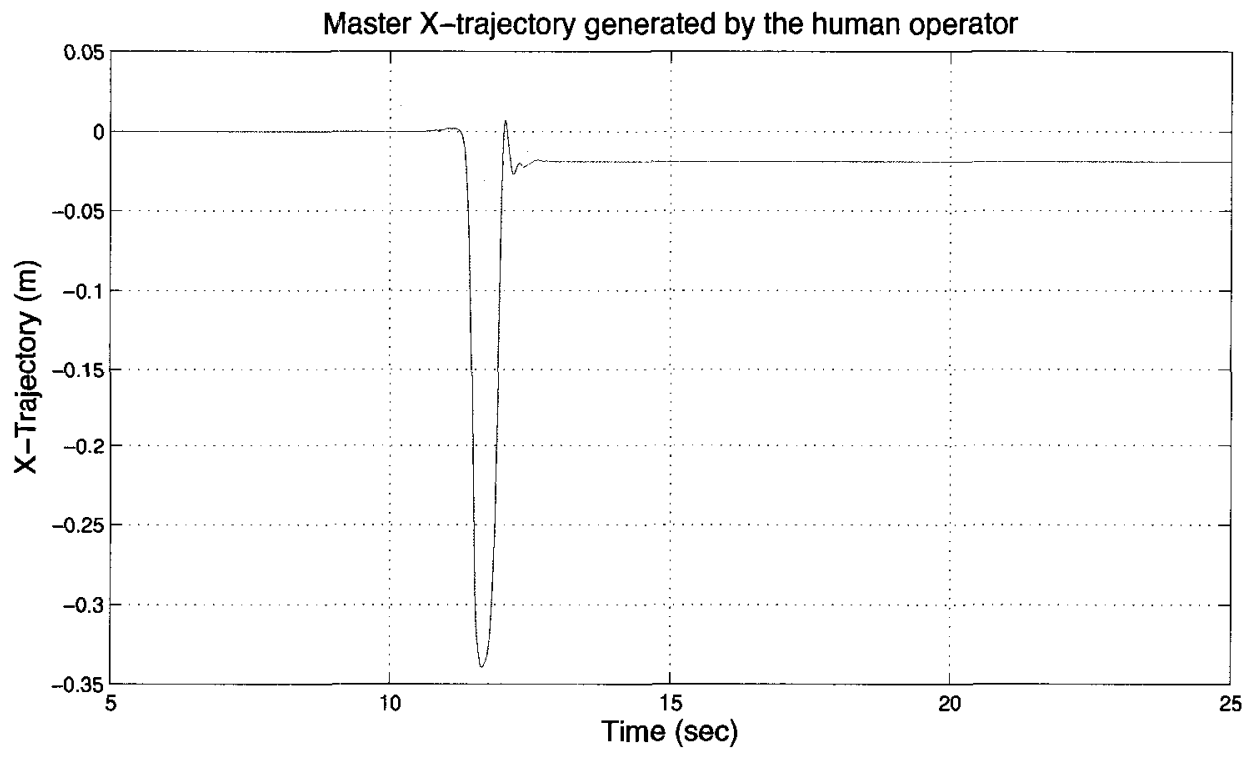

Figure 3.2: Example of the master trajectory generated by the human operator

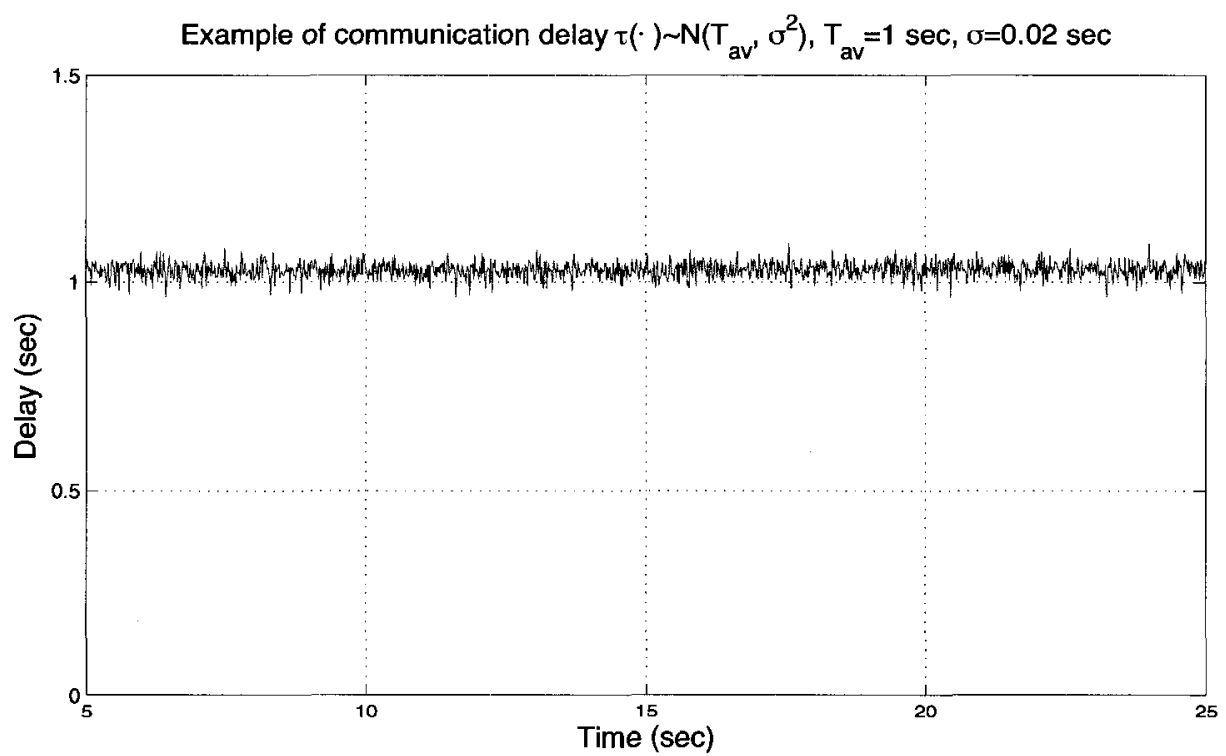

Figure 3.3: Example of communication delay function, $\tau(\cdot) \sim \mathbb{N}\left(T_{a v}, \sigma^{2}\right), T_{a v}=1$ sec, $\sigma=0.02 \mathrm{sec}$ 

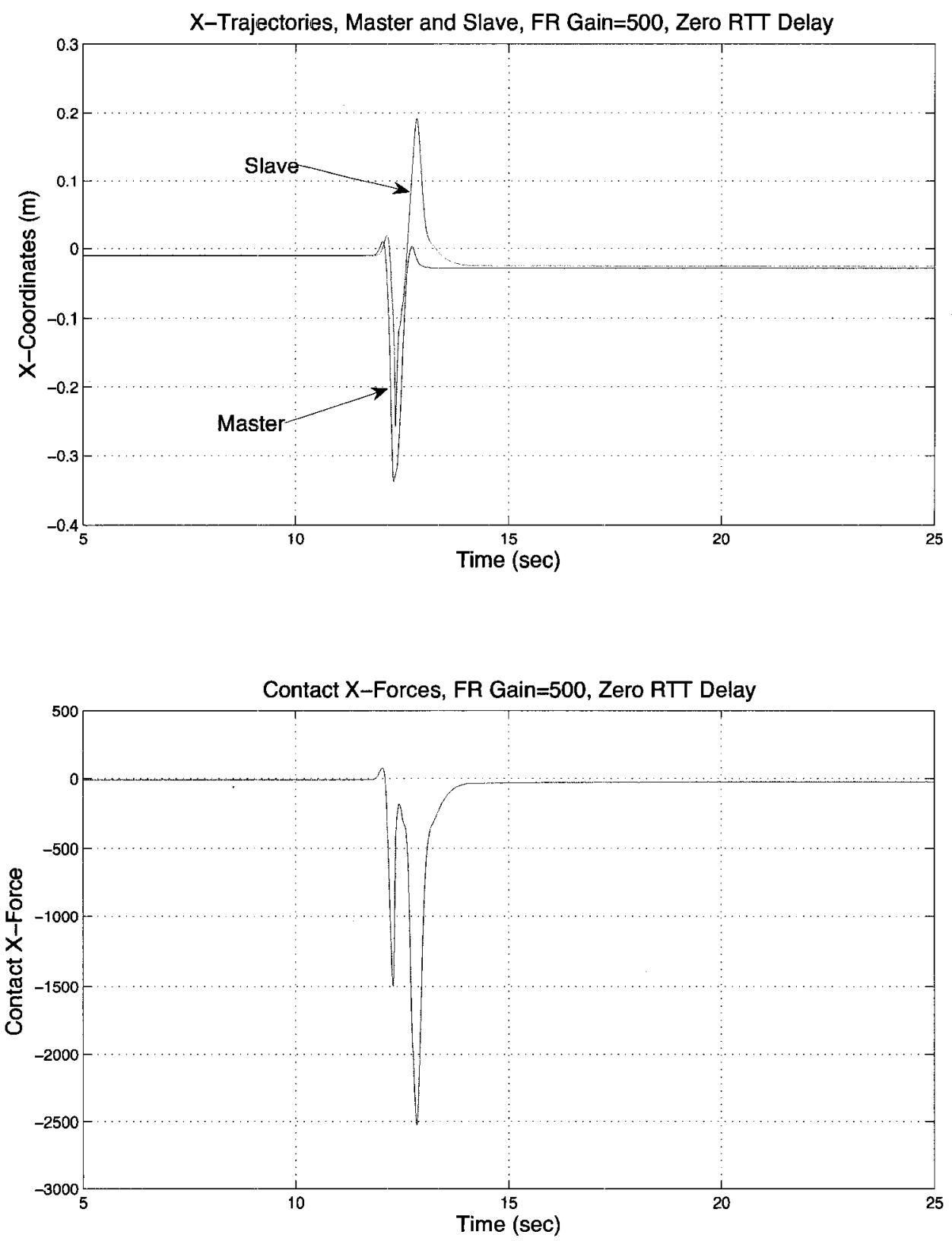

Figure 3.4: Teleoperator system response, Delay $\approx 0, \mathrm{FR}$ gain $=500$ : X-trajectories, master and slave (top); Contact X-Forces (bottom) 

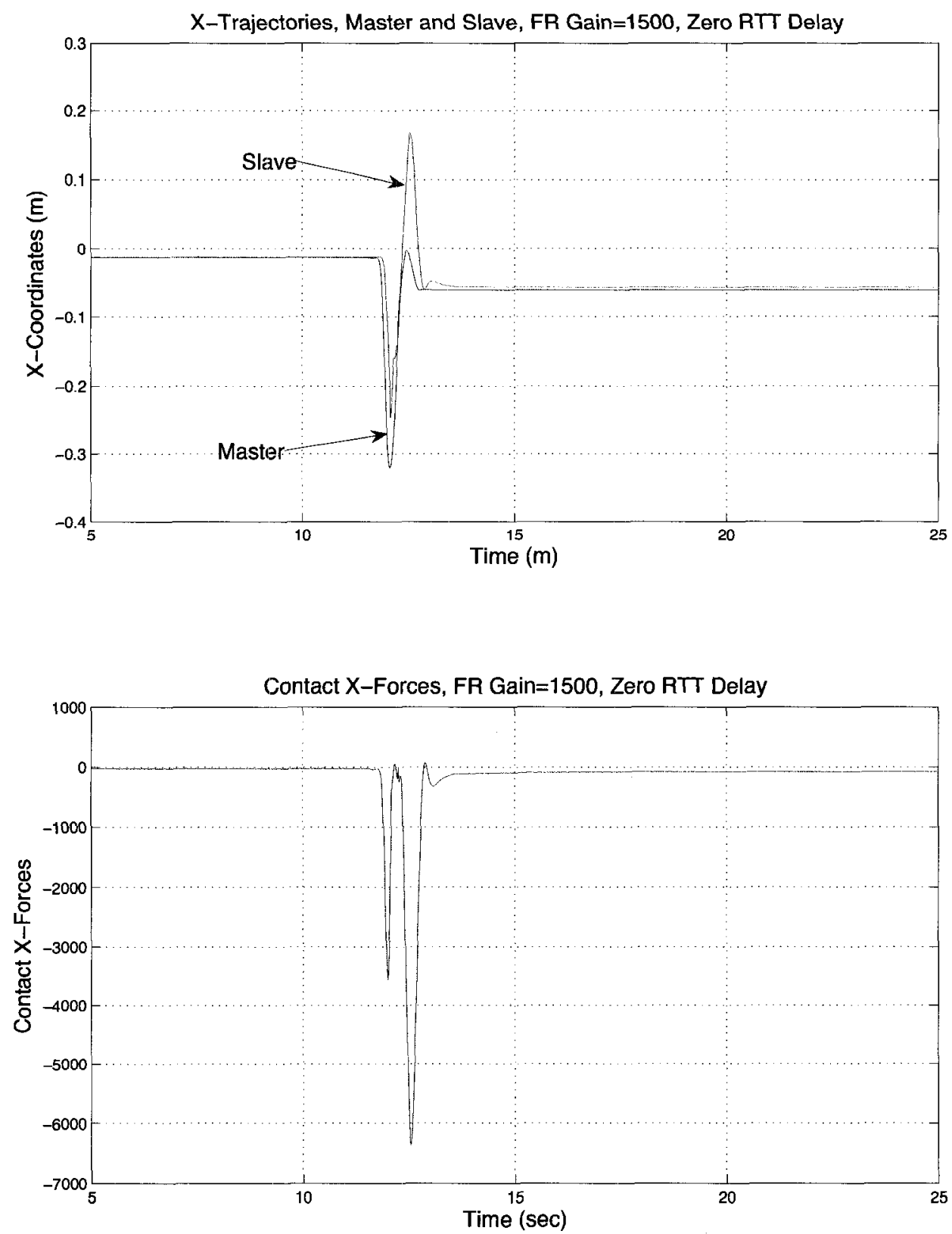

Figure 3.5: Teleoperator system response, Delay $\approx 0, \mathrm{FR}$ gain=1500: X-trajectories, master and slave (top); Contact X-Forces (bottom) 

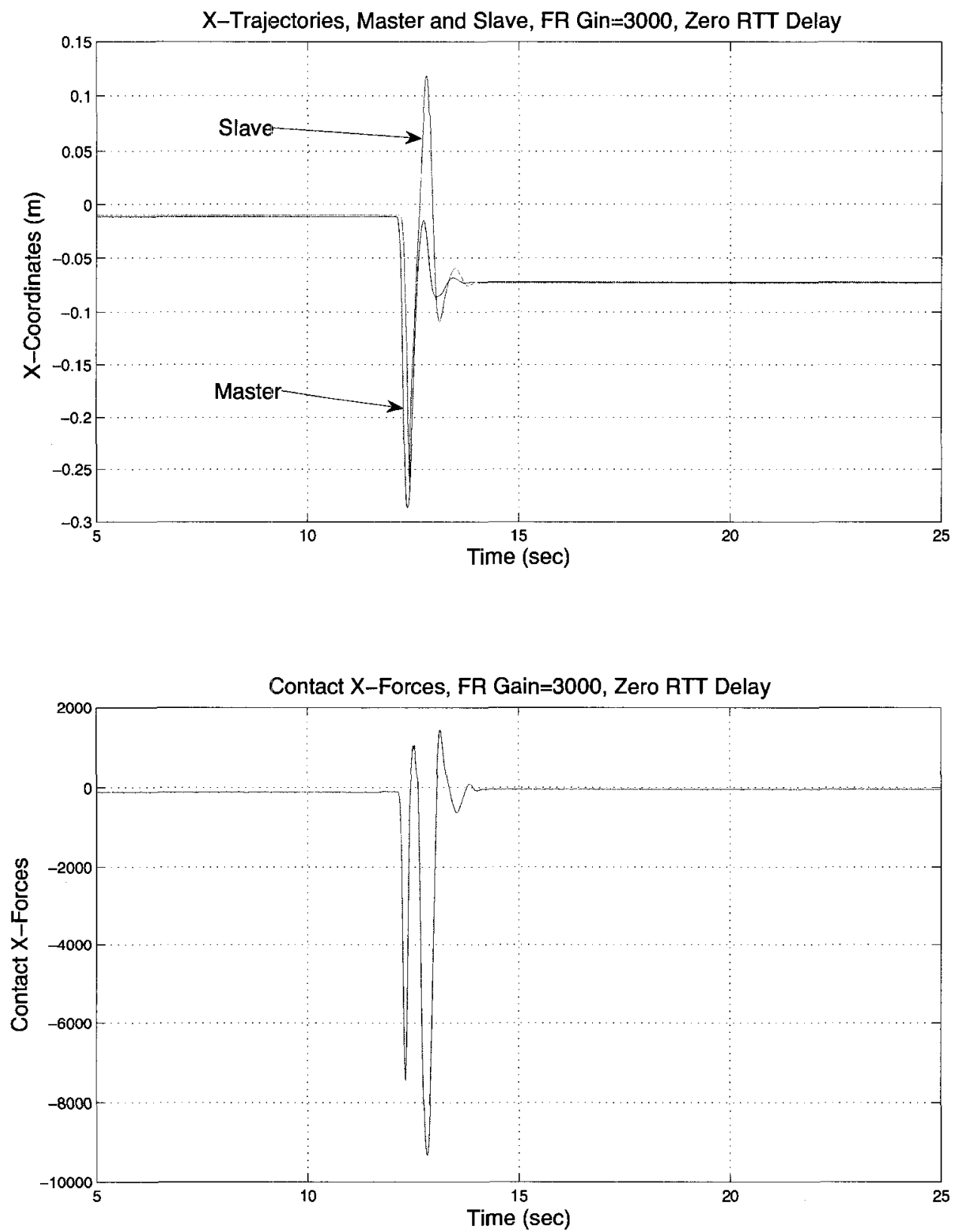

Figure 3.6: Teleoperator system response, Delay $\approx 0, \mathrm{FR}$ gain $=3000$ : $\mathrm{X}$-trajectories, master and slave (top); Contact X-Forces (bottom) 

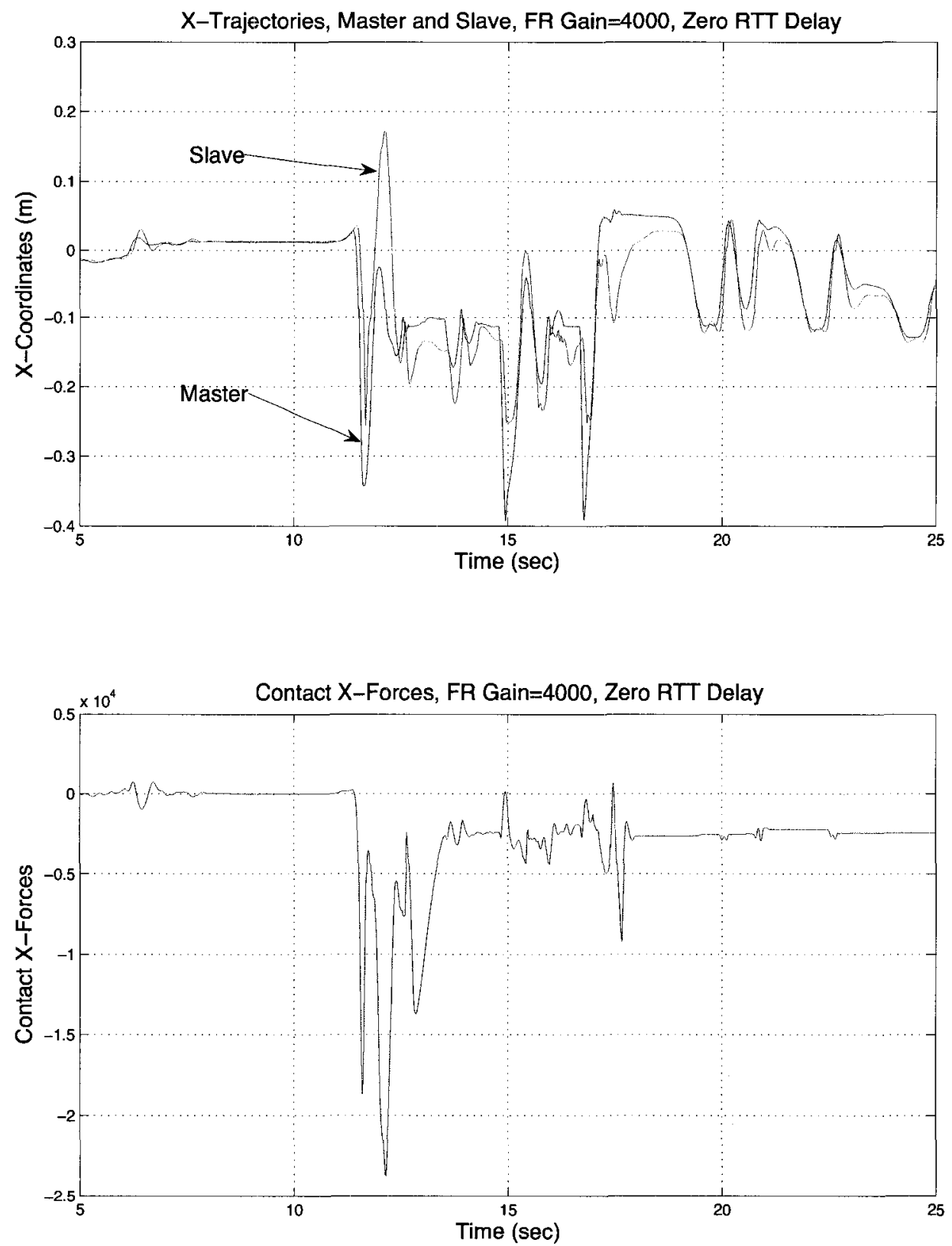

Figure 3.7: Teleoperator system response, Delay $\approx$ 0, FR gain=4000: X-trajectories, master and slave (top); Contact X-Forces (bottom) 

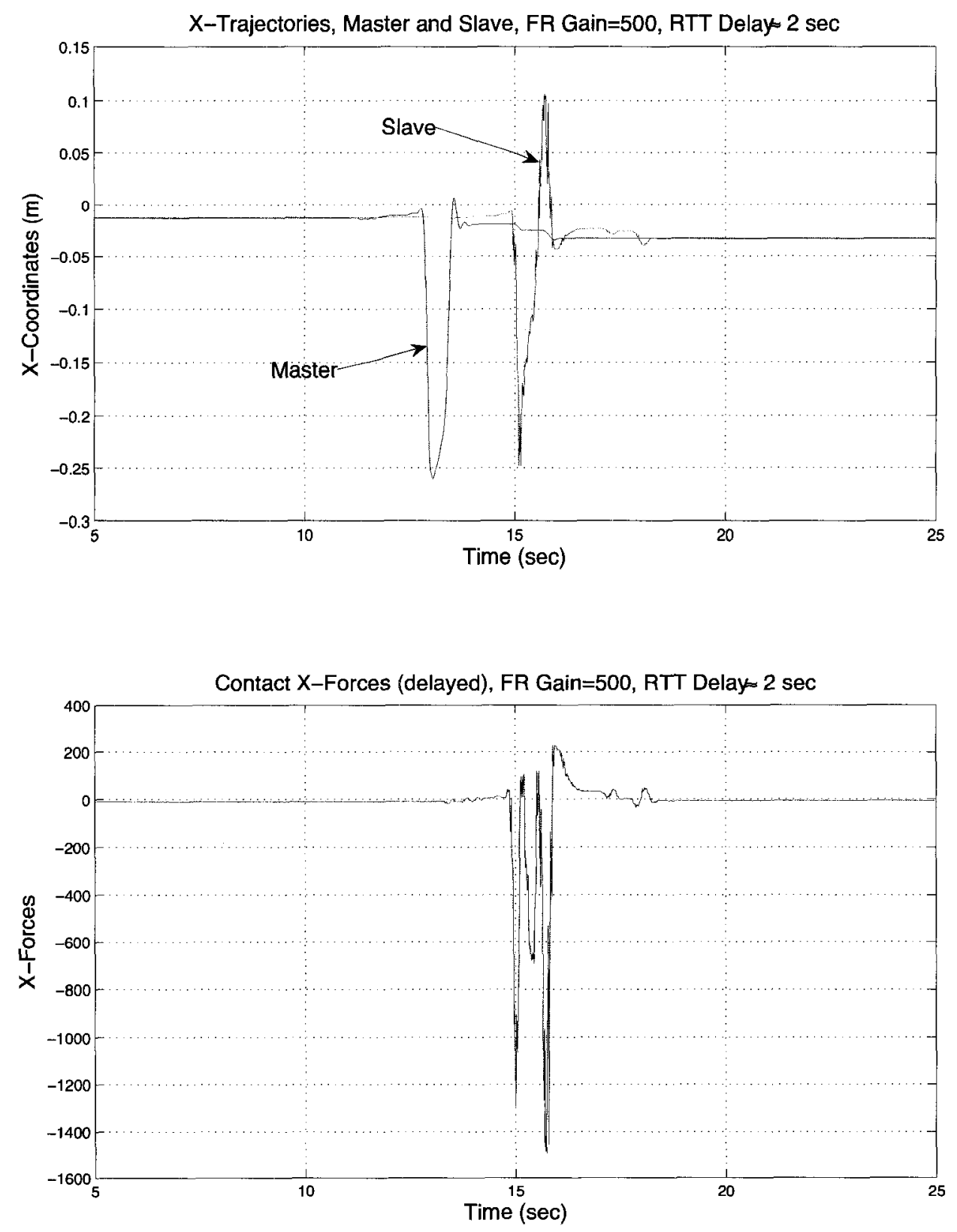

Figure 3.8: Teleoperator system response, Delays $\tau_{f}(\cdot), \tau_{b}(\cdot) \sim \mathbb{N}\left(T_{a v}, \sigma^{2}\right), T_{a v}=1 \mathrm{~s}$, $\sigma=0.02 \mathrm{~s}$, FR gain=500: X-trajectories, master and slave (top); Contact X-Forces (bottom) 

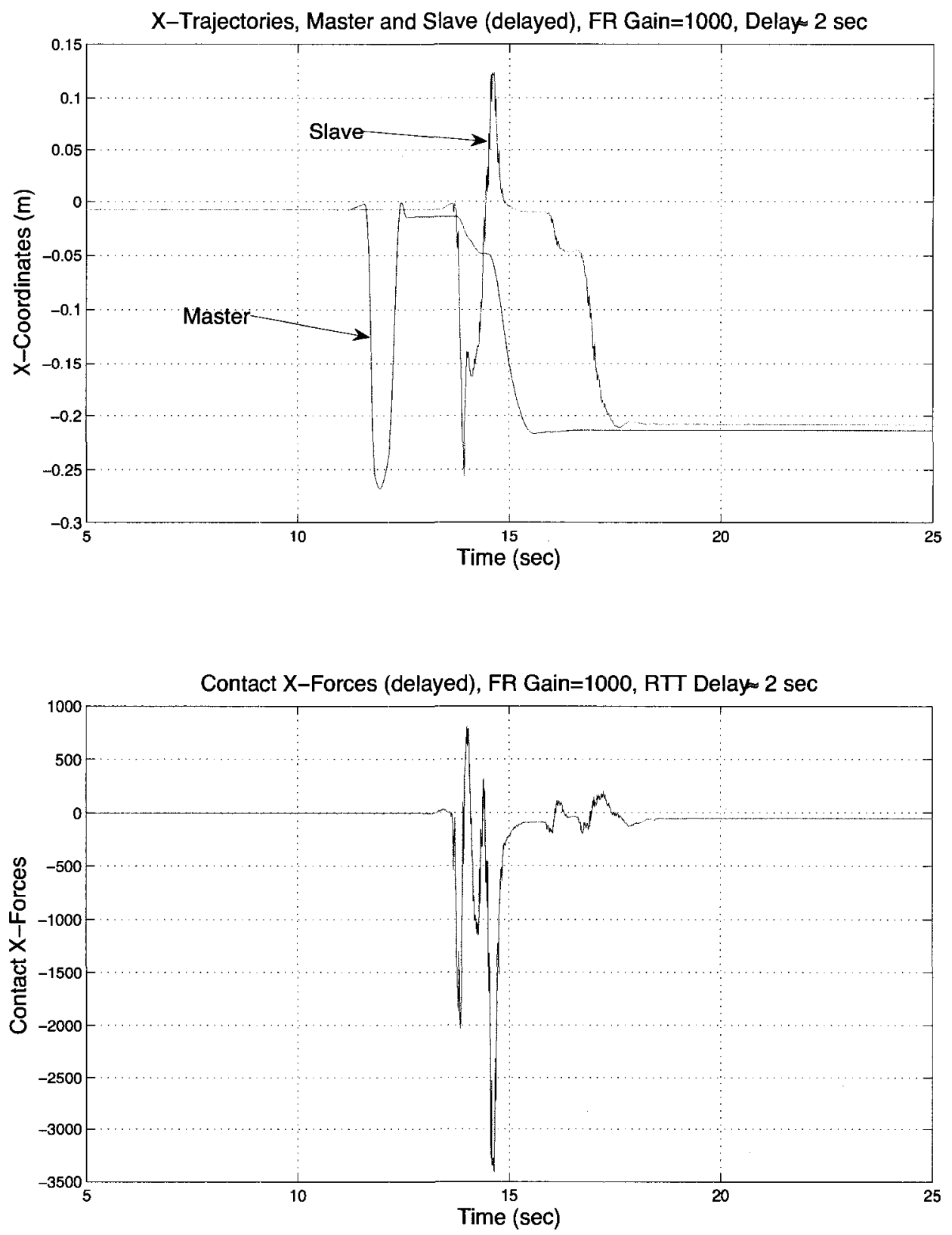

Figure 3.9: Teleoperator system response, Delays $\tau_{f}(\cdot), \tau_{b}(\cdot) \sim \mathbb{N}\left(T_{a v}, \sigma^{2}\right), T_{a v}=1 \mathrm{~s}$, $\sigma=0.02 \mathrm{~s}, \mathrm{FR}$ gain=1000: X-trajectories, master and slave (top); Contact X-Forces (bottom) 

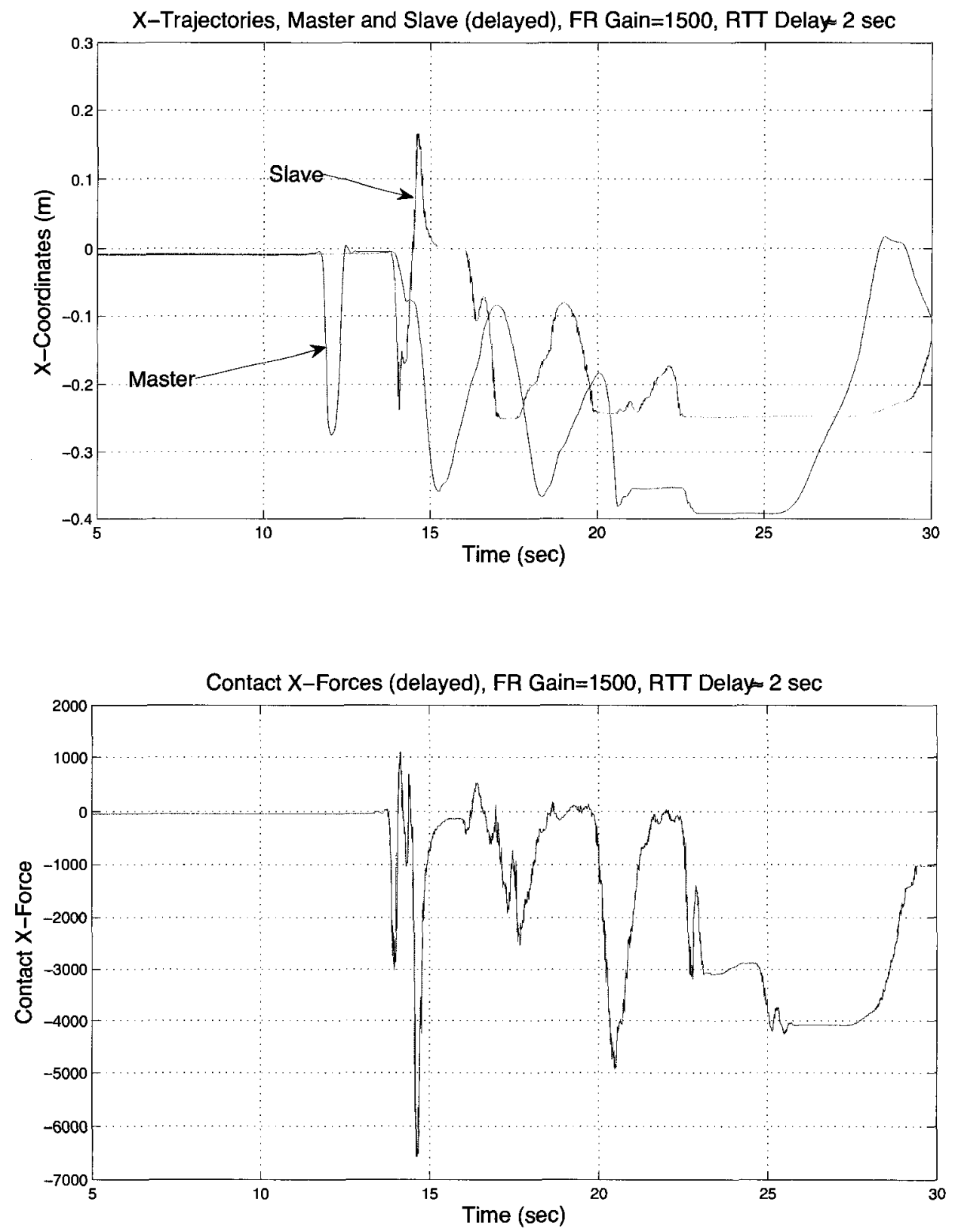

Figure 3.10: Teleoperator system response, Delays $\tau_{f}(\cdot), \tau_{b}(\cdot) \sim \mathbb{N}\left(T_{a v}, \sigma^{2}\right), T_{a v}=1$ s, $\sigma=0.02 \mathrm{~s}$, FR gain=1500: X-trajectories, master and slave (top); Contact XForces (bottom) 

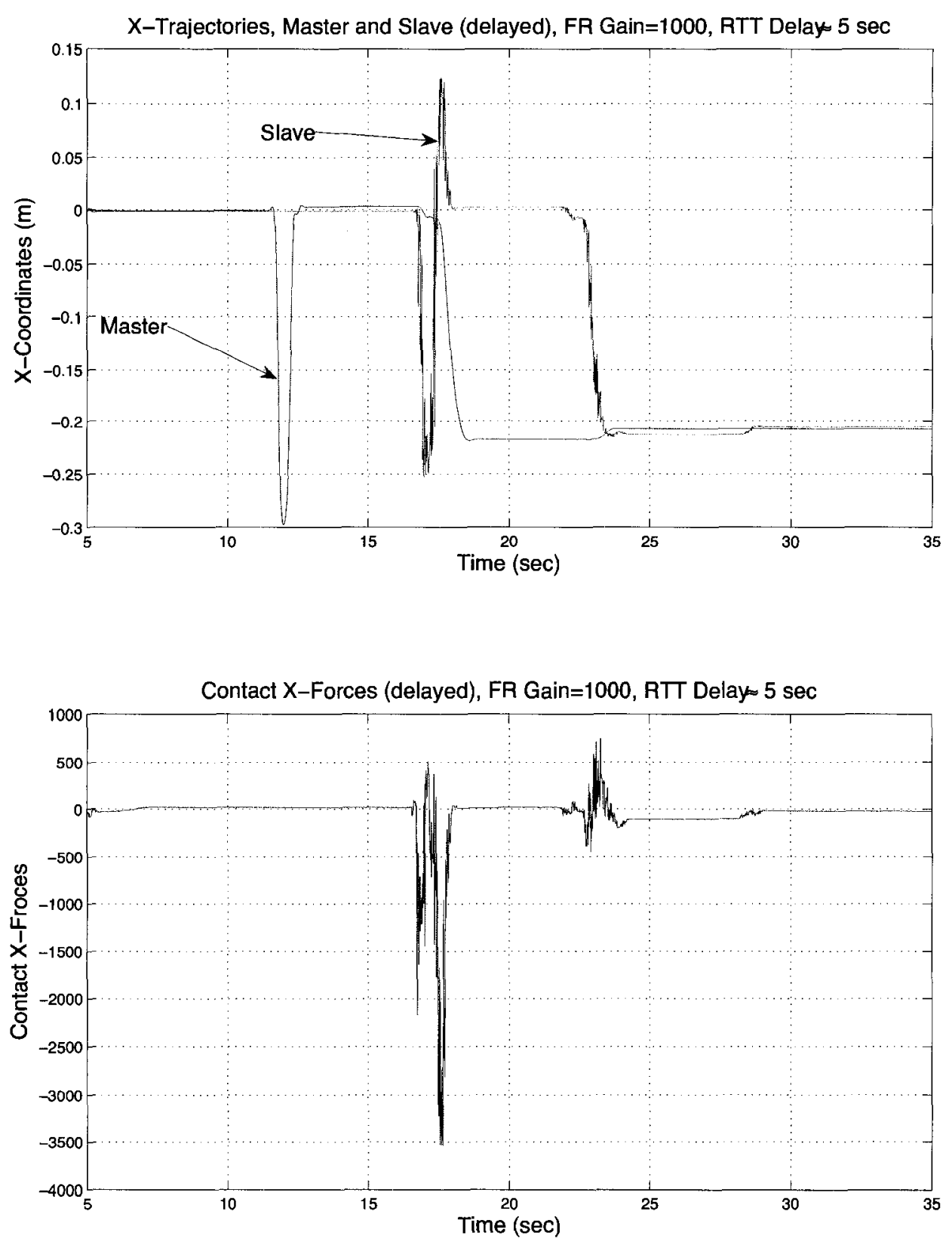

Figure 3.11: Teleoperator system response, Delays $\tau_{f}(\cdot), \tau_{b}(\cdot) \sim \mathbb{N}\left(T_{a v}, \sigma^{2}\right), T_{a v}=$ $2.5 \mathrm{~s}, \sigma=0.05 \mathrm{~s}, \mathrm{FR}$ gain=1000: X-trajectories, master and slave (top); Contact X-Forces (bottom) 


\section{Chapter 4}

\section{Projection-Based Force Reflection}

\section{Algorithms}

\subsection{Introduction}

This chapter address the problem of design of force-reflecting network-based teleoperator systems that achieve stable teleoperation in presence of high force reflecting gain. It is a well-known fact that, in bilateral teleoperation, stability and transparency are conflicting goals [15]. In particular, high force reflection gain provides a better kinesthetic as well as tactile (i.e., related to the sense of touch) feedback, however, it also tends to destabilize the overall system due to increasing the closedloop gain. To solve the contradiction between the overall stability and high force reflecting gain, we introduce an approach where the force reflecting term is shaped based on forces applied by the human operator. This approach uses a simple observation that a human operator can feel a force only when pushing against it, and the magnitude of the force felt by a human is exactly equal to the magnitude of the component of the external forces that is compensated by the human hand. Based 
on this observation, we introduce a class of projection-based force-reflection algorithms. The idea behind these algorithms is to attenuate the component of the external forces that is not compensated by the human hand; such an attenuation does not affect the human perception of the force, however, it removes the component of the force reflection that can potentially destroy the system's stability. The proposed approach allows to achieve the stability for an arbitrarily low damping of the master manipulator and arbitrarily high force reflection gain; thus, it solves the trade-off between stability, maneuvrability, and high force reflection gain in bilateral teleoperation with time-varying irregular communication delay.

The chapter is organized as follows. A trade-off between stability and high force reflection gain in bilateral teleoperation with communication delay is discussed in Section 4.2. In Section 4.3, projection-based force reflection algorithms are introduced. A general stability result for bilateral teleoperator systems with projectionbased force reflection algorithms is presented together with its proof in Section 4.4. In Section 4.5, we demonstrate that the use of projection-based force reflection algorithms leads to improved performance of the small-gain based schemes for bilateral teleoperation presented in Chapter 3. In Section 4.6, a human force estimator is designed that can be used in a projection-based force reflecting algorithm instead of direct measurement of the human force/torque. Experimental results are presented in Section 4.7, while in Section 4.8 some concluding remarks are given.

\subsection{Trade-Off Between Stability and High Force Reflection Gain}

The design of high performance teleoperator systems often involves trade-offs between conflicting design objectives. One such a trade-off that arises in force-reflecting 
teleoperators is between the overall stability and high force reflecting gain. Higher force reflecting gain generally implies improved haptic perception of the remote object, however, it also increases the closed-loop gain which leads to instability. The mechanism of such an instability is analyzed in great details in [66]. According to this work, the instability can be explained in terms of so called induced master motion. The induced master motion is the movement of the master manipulator due to the force signal reflected from the slave side. This movement results in deviation of the master trajectory from the one intended by the human operator, and since the master trajectory is then used as the reference trajectory for the slave manipulator, the induced master motion creates similar reaction of the slave subsystem, etc. Essentially, such an interaction forms a control loop, and the corresponding closed-loop gain is directly proportional to the force feedback gain; as a result, high force feedback gain leads to instability of the teleoperator system.

To the date, several approaches have been proposed in the literature to deal with trade-off between stability and high force reflection gain. The most obvious solution is to increase damping of the master manipulator using local feedback. This improve the stability of the system, however, the increased master damping itself leads to transparency deterioration, since in this case the human operator feels the stabilizing action of the local controller rather than the pure interaction with the remote environment. Increased master damping also makes the system "sluggish" and nonresponsive even when the slave moves in free space, and requires the human operator to apply excessive forces for manipulation. Other approaches include low-pass filtering of the slave reference trajectory [67] as well as low-pass filtering of the reflected force [68; 69]. Both of these approaches, however, also result in transparency deterioration. Filtering the reference trajectories prevents the slave from following quick master movements, which makes the system to be felt 
less responsive. Filtering of the reflected force, on the other hand, also decreases transparency, since the high-frequency band of the reflected force contains haptic information which is especially important during contact with hard objects.

Another approach, which has recently been proposed in [70], is based on the idea that the component of the master movement caused by the force reflection can be estimated and consequently canceled from the reference trajectory of the slave. Thus, the force reflection term is applied to the motors of the master and therefore felt by the human operator, however, the corresponding master reaction is removed from the feedback loop, thus avoiding possible instability. This approach, nevertheless, has at least two shortcomings. First, effective decomposition of the master movement into the one caused by the human force and the one due to force reflection is generally impossible when dealing with a nonlinear model of the manipulator. Second, some fundamental characteristics of the human response to the force disturbances are not taken into account in this approach. Indeed, as shown experimentally in [71], human tends to increase the impedance (stiffness as well as damping) of the muscles in presence of force disturbances while performing the mechanical tasks like moving a manipulator. This will result in that the increased damping and stiffness of the human hand will compensate, to a certain extent, the reflected forces, and the resulting deviation of the master trajectory will be smaller than the one calculated using the master model. The subsequent cancelation of the calculated master reaction from the slave reference trajectory, therefore, may lead to unpredictable movements of the slave and possible instability.

In the next section, we introduce the projection-based force reflection algorithms that solve the trade-off between stability and high force reflection gain without having any of the shortcomings mentioned above. 


\subsection{Projection-Based Force Reflection Algorithms}

The idea behind the projection-based force reflection algorithms is based on the following simple observation: the force felt by the human operator is exactly the component of the external forces that is compensated by the human hand. It is clear that, since this component of the external force is compensated, it does not create the induced master motion. On the contrary, the induced master motion is created by the residual component of the external forces which is not compensated by the human hand counter-force and, therefore, not immediately felt by the human operator. The combination of these observations leads us to the idea that the force reflection term may be altered depending on the forces applied by the human operator. More precisely, since the human operator feels the forces that are directed against her/his own, and the magnitude of the forces felt is not greater than the magnitude of the human forces exserted, this implies that all the external forces outside these direction and magnitude constraints can be attenuated or, in the ultimate case, completely filtered out; such an alteration will not be felt by the human operator, however, the potentially harmful induced master motion may be avoided or, at least, limited to an appropriate level that does not destroy the overall stability.

Below, we address a force reflection scheme where the force signal applied to the motors of the master $\hat{f}_{r}$ is described by the following formula

$$
\hat{f}_{r}=\frac{\alpha\left(\left|\hat{f}_{e n v}\right|\right)}{\left|\hat{f}_{e n v}\right|} \hat{f}_{e n v}+\frac{[\mathbb{I}-\alpha]\left(\left|\hat{\phi}_{e n v}\right|\right)}{\left|\hat{\phi}_{e n v}\right|} \hat{\phi}_{e n v}
$$

Here, $\hat{f}_{e n v}$ is the force signal that is arrived directly from the slave subsystem, $\hat{\phi}_{e n v}$ is the signal generated by the projection-based force reflection algorithm described below, and $\alpha \in \mathcal{G}$ is the corresponding weighting function; the last $\alpha$ should be chosen to satisfy $[\mathbb{I}-\alpha] \in \mathcal{G}$, where $\mathbb{I}: \mathbb{R}_{+} \rightarrow \mathbb{R}_{+}$is the identity function, $\mathbb{I}(r)=r$ 
for all $r \geq 0$. The signall $\hat{\phi}_{\text {env }}$ can be obtained using the force reflection algorithm, as follows

$$
\hat{\phi}_{e n v}:=\operatorname{Sat}_{[0,1]}\left\{\frac{\hat{f}_{e n v}^{T} \bar{f}_{h}}{\max \left\{\left|\bar{f}_{h}\right|^{2}, \epsilon_{1}\right\}}\right\} \bar{f}_{h},
$$

where $\vec{f}_{h}$ is a measurement/estimate of the human force applied to the master manipulator, $\epsilon_{1}>0$ is a sufficiently small constant, and

$$
\underset{[a, b]}{\operatorname{Sat}}\{x\}:= \begin{cases}a & \text { if } a \geq x \\ x & \text { if } a \leq x \leq b \\ b & \text { if } x \geq b .\end{cases}
$$

Algorithm (4.2) can be explained as follows. Assuming $\left|\bar{f}_{h}\right|^{2} \geq \epsilon_{1}$ and $0 \leq \hat{f}_{\text {env }}^{T} \bar{f}_{h} /\left|\bar{f}_{h}\right|^{2} \leq 1$ (i.e., the saturation in (4.2) is not achieved), it is easy to see that $\hat{\phi}_{e n v}$ is the projection of $\hat{f}_{e n v}$ onto the direction of $\bar{f}_{h}$. By placing the lower saturation limit at 0 , one guarantees that $-\hat{\phi}_{e n v}$ and $\bar{f}_{h}$ are directed against each other; on the other hand, the upper saturation limit at 1 implies that $\left|\hat{\phi}_{\text {env }}\right|$ does not exceed $\left|\bar{f}_{h}\right|$ (i.e., $\left.\left|\hat{\phi}_{\text {env }}\right| \leq\left|\bar{f}_{h}\right|\right)$. Finally, sufficiently small $\epsilon_{1}>0$ removes the singularity at $\bar{f}_{h}=0$. Thus, algorithm (4.2) calculates the component of the environmental force that is directed against the human force, and makes its magnitude bounded by the magnitude of the human force. Therefore, according to the considerations presented above, substitution of $\hat{\phi}_{e n v}$ for $\hat{f}_{e n v}$ in the force reflection scheme would not make a difference in terms of the human force sensing; however, contrary to the latter, the former does not generate the induced master motion.

The above presented algorithm calculates $\hat{\phi}_{e n v}$ as the projection of $\hat{f}_{e n v}$ onto the subspace spanned by the human force estimate $\bar{f}_{h}$; as a result, $\hat{\phi}_{e n v}$ is always collinear to $\bar{f}_{h}$. It is possible to construct another similar force reflection algorithm, where the resulting vector $\hat{\phi}_{e n v}$ would preserve the direction of the environmental force $\hat{f}_{\text {env }}$, however, its magnitude would depend on the magnitude of the projection 
of $\bar{f}_{h}$ onto the subspace spanned by $\hat{f}_{e n v}$. Such an algorithm is described by the formula

$$
\hat{\phi}_{e n v}:=\operatorname{Sat}_{[0,1]}\left\{\frac{\hat{f}_{e n v}^{T} \bar{f}_{h}}{\max \left\{\left|\hat{f}_{e n v}\right|^{2}, \epsilon_{1}\right\}}\right\} \hat{f}_{e n v} .
$$

Note that algorithms (4.2) and (4.3) give the same result if $\hat{f}_{e n v}$ is collinear to $\bar{f}_{h}$. It is also possible to use any convex combination of the algorithms (4.2) and (4.3).

Remark 7. It can be easily checked that $\hat{\phi}_{e n v}$ generated by any of the algorithms (4.2), (4.3), satisfies the inequality

$$
\left|\bar{f}_{h}-\hat{\phi}_{\text {env }}\right| \leq\left|\bar{f}_{h}\right| .
$$

This property will be utilized below, where we formulate and prove stability results for systems with force-reflecting algorithms that satisfy an inequality more general that (4.4).

\subsection{General Stability Result}

In this section, we address stability properties of bilateral teleoperator system with force reflection algorithms described above. More precisely, we define a class of force reflection algorithms that, in particular, includes algorithms (4.2) and (4.3), and prove general stability result for teleoperator system with any force reflection algorithm from that class.

We assume that the closed-loop "master manipulator plus local master controller" subsystem is described as a general nonlinear system of the form

$$
\begin{aligned}
& \dot{x}_{m}=F_{m}\left(x_{m}, u_{m}\right), \\
& y_{m}=G_{m}\left(x_{m}, u_{m}\right),
\end{aligned}
$$

where $x_{m}$ is the state of the master subsystem, and $u_{m}$ is the master input. We impose general regularity assumptions on $F_{m}$ and the output map $G_{m}$; namely, it 
is assumed that both $F_{m}(\cdot, \cdot), G_{m}(\cdot, \cdot)$ are locally Lipschitz in their arguments. The input of the master subsystem is the external force input

$$
u_{m}=f_{h}-\hat{f}_{r},
$$

where $f_{h}$ is the force/torque applied by the human operator, and $\hat{f}_{r}$ is the force/torque reflection signal. The output $y_{m}$ of the master subsystem contains an arbitrary set of signals that are to be transmitted to the slave side, these may consist of master positions, velocities, forces/torques, as well as arbitrary combinations of them. During the transmission, the output $y_{m}$ is subject to time-varying communication delay $\tau_{f}: \mathbb{R}_{+} \rightarrow \mathbb{R}_{+}$; the transmitted version of the master output is then applied to the input of the slave subsystem, as follows

$$
u_{s}(t):=y_{m}\left(t-\tau_{f}(t)\right) .
$$

The closed loop "slave plus environment plus local slave controller" subsystem is also described as a nonlinear system of the form similar to (3.1), i.e.,

$$
\begin{aligned}
& \dot{x}_{s}=F_{s}\left(x_{s}, u_{s}\right), \\
& y_{s}=G_{s}\left(x_{s}, u_{s}\right),
\end{aligned}
$$

where $x_{s}$ is a state of the slave+environment interconnection, $u_{s}$ is the input, and $y_{s}$ is the output of the slave subsystem. Again, both $F_{s}$ and $G_{s}$ are assumed to be locally Lipschitz functions of $x_{s}, u_{s}$. The output $y_{s}$ of the slave subsystem is again an arbitrary force signal which is to be transmitted to the master subsystem. This force signal may contain information about contact forces due to environment, position errors, velocity errors, or any other signals that depend on the state of the slave+environment subsystem or its inputs. Last but not least, these signals can be multiplied by arbitrary coefficients, which, in particular, corresponds to an arbitrary force reflection gain. The transmission of $y_{s}$ to the master side is also a subject to 
time-varying communication delay $\tau_{b}: \mathbb{R}_{+} \rightarrow \mathbb{R}_{+}$, according to the formula

$$
\hat{f}_{e n v}(t):=y_{s}\left(t-\tau_{b}(t)\right)
$$

Based on $\hat{f}_{e n v}$, the force reflection signal $\hat{f}_{r}$ in (4.6) is then generated according to the formula (4.1), where $\hat{\phi}_{e n v}$ is the outcome of a projection-based force reflection algorithm (such as (4.2) or (4.3)).

In order to formulate and prove a general stability result for teleoperator system with projection-based force reflection, a number of assumptions is imposed on the system (4.5)-(4.9). First, the input-to-state stability of both the master and the slave subsystems is assumed, as follows.

Assumption 5. Both the master (4.5) and the slave (4.8) subsystems are input-to-state stable in the sense of Definition 1 (with $l=r=1$ and $t_{d}(\cdot) \equiv 0$ ).

Remark 8. Due to regularity (local Lipschitzness) assumption imposed on $G_{m}$, $G_{s}$, the input-to-state stability also implies the input-to-output stability of both the master and the slave subsystems. Note that, contrary to Assumption 2 which is used in the proof of the general small-gain result (Theorem 1), neither ISS nor IOS gains are specified in Assumption 5; only the existence of these gains is assumed. As a result, such an assumption allows significant flexibility in design of local controllers for both the master and the slave subsystems; in particular, the ISS property (with possibly high ISS gain) of the master subsystem can be achieved, for example, by using local PD controller with arbitrarily low damping and stiffness coefficients [62].

Second assumption is imposed on communication delays $\tau_{f}(\cdot), \tau_{b}(\cdot)$; this is essentially an "one-channel" version of Assumptions 1.

Assumption 6. The communication delays $\tau_{f}, \tau_{b}: \mathbb{R}_{+} \rightarrow \mathbb{R}_{+}$satisfy Assumption 1 (with $T_{0}=0$ and $p=q=1$ ). 
The next assumption describes the class of the force reflecting algorithms. The assumption is a generalization of the property described above in Remark 7.

Assumption 7. There exists $\eta \in \mathcal{K}$ such that $\hat{\phi}_{\text {env }}$ in (4.1) satisfies the inequality

$$
\left|\bar{f}_{h}-\hat{\phi}_{e n v}\right| \leq \eta\left(\left|\bar{f}_{h}\right|\right)
$$

Note that, according to Remark 7, both the projection-based force reflection algorithms (4.2) and (4.3) satisfy Assumption 7 with $\eta(\cdot) \equiv \mathbb{I}(\cdot)$.

The fourth and last assumption is related to the force measurement/estimation process on the master side. In the results presented below, we do not restrict our consideration to the situation where the human force/torque is perfectly measured. On the contrary, we consider the human force measurement/estimation as a process whose accuracy may depend on a values of the human force and its derivatives as well as on disturbance level. More precisely, a sort of input-to-state stability assumption is imposed on the human force measurement/estimation process, as follows.

Assumption 8. Human force (torque) measurement/estimation process satisfies the following estimate

$$
\left|f_{h}(t)-\widetilde{f}_{h}(t)\right| \leq \max \left\{\begin{array}{l}
\beta\left(\left|f_{h}(0)-\bar{f}_{h}(0)\right|, t\right), \\
\gamma_{f}^{\{0\}}\left(\sup _{s \in[0, t)}\left|f_{h}(s)\right|\right), \ldots, \\
\gamma_{f}^{\{r\}}\left(\sup _{s \in[0, t)}\left|f_{h}^{\{r\}}(s)\right|\right), \\
\gamma_{w}\left(\sup _{s \in[0, t)}|w(s)|\right)
\end{array}\right\}
$$

for all $t \geq 0$, where $f_{h}$ is the human force applied to the master, $f_{h}^{\{i\}}$ is its i-th derivative, $w$ are the external disturbances that affect the measurement process, $\beta_{m} \in \mathcal{K} L, \gamma_{f}^{\{0\}}, \ldots,{ }^{\{r\}}, \gamma_{w} \in \mathcal{K}$.

Remark 9. Assumption 8 allows to address schemes where the direct input force measurement is performed in presence of sensor noise as well as a wide range of schemes that utilize different input estimation techniques [72]. In particular, 
the specific form of inequality (4.11) is motivated by the fact that the accuracy of estimates provided by input observers normally depends on derivatives of input (first derivative in the cases of high-gain and sliding mode input observers, second derivative for "dirty-derivative" filters, etc.)

Consider now the closed-loop teleoperator system (4.5)-(4.9). Our main stability result can be formulated as follows.

Theorem 6. Consider the closed-loop force reflecting teleoperator system (4.5)(4.9) with force reflection algorithm (4.1). Suppose Assumptions 5-8 are satisfied. Then there exists $\alpha_{*} \in \mathcal{K}_{\infty}$ such that if $\alpha(\cdot) \in \mathcal{G}$ in (4.1) satisfies $\alpha(s) \leq \alpha_{*}(s)$ for all $s \geq 0$ then the closed-loop force reflecting teleoperator system with state

$$
\mathbf{x}_{d}=\left(x_{m}^{T}, x_{s}^{T}\right)_{d}^{T}
$$

and input

$$
\mathbf{u}=\left(f_{h}^{T}, \ldots,\left(f_{h}^{\{r\}}\right)^{T}, w^{T}\right)^{T}
$$

is input-to-state stable in the sense of Definition 1 with $t_{d}$ defined by (3.17).

Proof of Theorem 6 is based on a version of a small-gain theorem for systems with delays presented in Chapter 3. First, let us note that

$$
\left|u_{m}\right|=\left|f_{h}-\hat{f}_{r}\right| \leq\left|f_{h}-\bar{f}_{h}\right|+\left|\bar{f}_{h}-\hat{f}_{r}\right|
$$

Assuming without loss of generality that $\eta(\cdot)$ in $(4.10)$ satisfies $\eta(s) \geq \mathbb{I}(s)$ for all $s \geq 0$, one gets

$$
\begin{gathered}
\left|\bar{f}_{h}-\hat{f}_{r}\right|=\left|\bar{f}_{h}-\frac{\left.\alpha\left(\left|\hat{f}_{e n v}\right|\right)\right)}{\left|\hat{f}_{e n v}\right|} \hat{f}_{e n v}-\frac{\left[\mathbb{I}-\alpha \mid\left(\left|\hat{\phi}_{e n v}\right|\right)\right.}{\left|\hat{\phi}_{e n v}\right|} \hat{\phi}_{e n v}\right| \leq \alpha\left(\left|\hat{f}_{e n v}\right|\right)+\left|\bar{f}_{h}-\frac{[\mathbb{I}-\alpha]\left(\left|\hat{\phi}_{e n v}\right|\right)}{\left|\hat{\phi}_{e n v}\right|} \hat{\phi}_{e n v}\right| \\
\leq \alpha\left(\left|\hat{f}_{e n v}\right|\right)+\eta\left(\left|\bar{f}_{h}\right|\right) \leq \alpha\left(\left|\hat{f}_{e n v}\right|\right)+\eta\left(2\left|f_{h}-\bar{f}_{h}\right|\right)+\eta\left(2\left|f_{h}\right|\right) .
\end{gathered}
$$

(In the sequence above, the inequality

$$
\left|\bar{f}_{h}-\frac{[\mathbb{I}-\alpha]\left(\left|\hat{\phi}_{e n v}\right|\right)}{\left|\hat{\phi}_{e n v}\right|} \hat{\phi}_{e n v}\right| \leq \eta\left(\left|\bar{f}_{h}\right|\right)
$$


follows from (4.10) because $[\mathbb{I}-\alpha]\left(\left|\hat{\phi}_{e n v}\right|\right) \leq\left|\hat{\phi}_{e n v}\right|$, and $|\cdot|$ is a convex function). Overall,

$$
\left|u_{m}\right| \leq \alpha\left(\left|\hat{f}_{e n v}\right|\right)+\bar{\eta}\left(\left|f_{h}-\bar{f}_{h}\right|\right)+\eta\left(2\left|f_{h}\right|\right),
$$

where $\vec{\eta}(s):=\eta(2 s)+s$. Taking into account (4.11), one sees that the gain from $\left|\hat{f}_{\text {env }}\right|$ to $\left|u_{m}\right|$ is less than or equal to $\alpha(\cdot)$. Now, let $\gamma_{m}, \gamma_{s} \in \mathcal{K}$ be IOS gains of the master (3.1) and the slave (3.2) subsystems, respectively. From (4.7), (4.9), it follows that the closed loop system gain is $\gamma_{m} \circ \gamma_{s} \circ \alpha(\cdot)$, and therefore, the small-gain stability condition for the system under consideration has a form

$$
\gamma_{m} \circ \gamma_{s} \circ \alpha(s)<\mathbb{I}(s) \text { for all } s>0
$$

The condition (4.12) is met if $\alpha(s) \leq \alpha_{*}$, where

$$
\alpha_{*}^{-1}(s):=\gamma_{m} \circ \gamma_{s}(s)+s
$$

The statement of Theorem 6 now follows from the small gain result (Theorem 1).

One of the most interesting features of the result presented in the above Theorem 6 is that it does not impose any restrictions on the ISS (IOS) gains of the master and slave subsystem. Instead, given the master and the slave gains, the overall stability can always be achieved by an appropriate choice of the weighting function $\alpha(\cdot) \in \mathcal{G}$ in (4.1). As one can see from the formula (4.1), $\alpha(\cdot) \in \mathcal{G}$ and $[\mathbb{I}-\alpha](\cdot) \in \mathcal{G}$ determine the relative weights of the terms $\hat{f}_{\text {env }}$ and $\hat{\phi}_{\text {env }}$, respectively, in the force reflection signal $\hat{f}_{r}$. Both these terms provide the haptic feedback to the operator; however, the difference between them is that, contrary to $\hat{f}_{e n v}$, the term $\hat{\phi}_{e n v}$ does not create the induced master motion that can potentially destabilize the overall teleoperator system. Thus, the choice of $\alpha(\cdot)$ determines the gain between the force reflection signal and the resulting induced master motion. In particular, if $\alpha(\cdot) \equiv 0$ in $(4.1)$, the induced master motion is eliminated completely; in this case, 
the stability is guaranteed simultaneously for all gains as long as both the master and the slave subsystems are stable. Thus, the design of stable force reflecting teleoperator system with communication delay is essentially reduced to the design of two stable subsystems. Theoretically, the choice $\alpha(\cdot) \equiv 0$ disconnects the feedback loop in terms of induced master motion; i.e., the corresponding closed loop gain becomes equal to zero. In particular, this allows to achieve stability for arbitrarily low damping and stiffness of the master manipulator and in presence of arbitrarily high force reflection gain, which has numerous advantages in terms of better operability and transparency. On the other hand, a nonzero $\alpha(\cdot)$ implies that the force reflection term may create some induced motion of the master manipulator; however, the amount of this motion is not sufficient to destroy the overall stability as long as $\alpha(\cdot)$ is small enough. It seerns conceivable that certain small enough amount of the induced master motion may be useful in some teleoperation tasks; for example, it may impel the otherwise inactive human operator to apply forces against it. It can also be noticed that a small amount of the induced master motion may improve force regulation.

\subsection{A Scheme with Direct Human Force Measure- ment}

The main purpose of this section is to show that the force-reflecting algorithms described above may significantly improve performance of the small-gain schemes for network-based teleoperation presented in Chapter 3. In particular, we show that, using the projection-based force reflecting algorithm, stability/tracking properties of the force-reflecting network-based teleoperators can be achieved for arbitrarily high force reflecting gain and arbitrarily low damping/stiffness of the master manipulator, 
which fundamentally improves transparency of the teleoperator system.

Consider a force-reflecting teleoperator system where the master and the slave manipulators are described by the Euler-Lagrange equations of the form (3.1), (3.2), respectively. The control schemes used in this section are the same as the ones used in Chapter 3. Namely, it is assumed that the master manipulator is equipped with local control algorithm described either by (3.5) or by (3.6), while the local slave controller consists of controller (3.7) and filter (3.8), (3.9). Also, it is assumed that the master position is sent over the communication channel to the remotely located slave according to the formula (3.3). The communication process in the forward communication channel is described by equation (3.3). Regarding the communication in the backward channel, it is convenient to change the notation slightly, as follows. Let the following signal

$$
f_{\text {env }}(t)=K_{f} y_{s}(t)
$$

be transmitted to the master side according to the formula

$$
\hat{f}_{e n v}(t):=f_{e n v}\left(t-\tau_{b}(t)\right)+\hat{\delta}_{f}(t)
$$

In the above expressions, $y_{s}$ is the "output" of the slave-environment subsystem defined by (3.10), $K_{f} \geq 0$ is the force reflection gain communication delay $\tau_{b}(t)$ is the communication delay in the backward channel, and $\hat{\delta}_{f}(t)$ is quantization/measurement noise error. The signal $\hat{f}_{\text {env }}$ is then processed by the force reflection algorithm $(4.1),(4.2)$ or $(4.1),(4.3)$, and the output $\hat{f}_{r}$ is reflected to the motors of the master according to (3.1). The state and the input of the closed-loop teleoperator system are defined by (3.15) and (3.16), respectively. The following theorem describes stability properties of the teleoperator system with projection-based force reflection algorithm. 
Theorem 7. Consider the system (3.1), (3.2), (3.3), (3.4), (3.11), (3.12), where the master control algorithm is described by either (3.5) or (3.6), and the slave control law is (3.7)-(3.9). Suppose the delays $\tau_{f}(\cdot), \tau_{b}(\cdot)$ in both the forward and the backward communication channels (3.3), (3.4) satisfy Assumption 1. Suppose also that the environmental dynamics (3.12) satisfy Assumption 3. Then, given $K_{f}^{*} \in[0,+\infty)$, there exists $\alpha^{*}(\cdot) \in \mathcal{K}_{\infty}$ such that if $\alpha(\cdot) \in \mathcal{G}$ in (4.1) satisfies $\alpha(s) \leq \alpha^{*}(s)$ for all $s \geq 0$, then the closed-loop teleoperator system is input-tostate stable at $t=0$ with $t_{d}$ defined by (3.17), for all $K_{f} \in\left[0, K_{f}^{*}\right)$.

Proof of Theorem 7 follows directly by combination of arguments from the proofs of Theorems 2, 3, and Theorem 6 .

Remark 10. Theorem 7 implies in particular that, if any of the projection-based force reflection algorithms (4.1), (4.2) or (4.1), (4.3) is utilized, the stability of the overall teleoperator system can be achieved for an arbitrarily low damping/stiffness on the master side (i.e., arbitrary $K_{m}>0, \Lambda_{m}>0$ ) and arbitrarily high force reflection gain (arbitrary $K_{f} \geq 0$ in (4.13)), by an appropriate choice of the weighting factor $\alpha$. Note that a higher force reflection gain provides stronger haptic cues to the human operator; on the other hand, higher master damping/stiffness lead to transparency deterioration since these forces interfere with environmental forces reflected. Thus, Theorem 7 implies, in particular, that the proposed force reflection algorithm substantially improves transparency of the teleoperation.

\subsection{A Scheme without Human Force Measure- ment}

The above presented FR algorithms utilize an estimate $\hat{f}_{h}$ of the force $f_{h}$ applied by the human operator to the master manipulator. If a direct force measurement 
on the master side is not available, $\hat{f}_{h}$ can be obtained using some sort of input estimation technique. Different types of input estimators can be employed. One possible approach is to construct a high gain input observer designed as follows [72]. Suppose we deal with a system of the form

$$
\dot{z}=y+u
$$

where $z$ and $y$ are known (measured) signals and $u$ is an unknown input to be estimated. Then, the following input observer

$$
\begin{aligned}
\dot{w} & =-\gamma w+\gamma y+\gamma^{2} z, \\
\bar{u} & =\gamma z-w,
\end{aligned}
$$

provides an estimate $\bar{u}$ of the unknown input $u$, where $w$ is an auxiliary variable, and $\gamma>0$ is an observer gain which determines the transient response as well the ultimate bound of the estimation error. More precisely, the estimate $\bar{u}$ satisfies the following inequality

$$
|\bar{u}(t)-u(t)| \leq \sqrt{|\bar{u}(0)-u(0)|^{2} e^{-\gamma t}+\frac{\sup _{s \in[0, t]}|\dot{u}(s)|^{2}}{\gamma^{2}}},
$$

which implies that the estimation error satisfies a form of the input-to-state stability property. In the case of the master manipulator described by (3.1), signals $z, y$, and $u$ can be chosen as follows: $z=H_{m}\left(q_{m}\right) \dot{q}_{m}, u=f_{h}$, and

$$
y=\dot{H}_{m}\left(q_{m}, \dot{q}_{m}\right) \dot{q}_{m}-C_{m}\left(q_{m}, \dot{q}_{m}\right) \dot{q}_{m}-G_{m}\left(q_{m}\right)-\hat{f}_{r}+u_{m},
$$

where $\dot{H}_{m}\left(q_{m}, \dot{q}_{m}\right) \in \mathbb{R}^{n \times n}, \dot{H}_{m}^{\{i j\}}\left(q_{m}, \dot{q}_{m}\right)=\left(\partial H_{m}^{\{i j\}}\left(q_{m}\right) / \partial q_{m}\right)^{T} \dot{q}_{m}, i, j \in\{1, \ldots, n\}$, and $u_{m}$ is the master control input determined by the control algorithm used. The above choice of $z, y, u$ results in the observer of the form

$$
\begin{aligned}
\dot{w}= & -\gamma w+\gamma\left(\dot{H}_{m}\left(q_{m}, \dot{q}_{m}\right) \dot{q}_{m}-C_{m}\left(q_{m}, \dot{q}_{m}\right) \dot{q}_{m}-G_{m}\left(q_{m}\right)-\hat{f}_{r}+u_{m}\right) 4 \\
& +\gamma^{2} H_{m}\left(q_{m}\right) \dot{q}_{m} \\
\bar{f}_{h}= & \gamma H_{m}\left(q_{m}\right) \dot{q}_{m}-w
\end{aligned}
$$


and estimate (4.16) becomes

$$
\begin{aligned}
& \left|\bar{f}_{h}(t)-f_{h}(t)\right| \leq \sqrt{\left|\bar{f}_{h}(0)-f_{h}(0)\right|^{2} e^{-\gamma t}+\gamma^{-2} \sup _{s \in[0, t]}\left|\dot{f}_{h}(s)\right|^{2}} \\
& \quad \leq \sqrt{2} \max \left\{\left|\bar{f}_{h}(0)-f_{h}(0)\right| e^{-\gamma t / 2}, \gamma^{-1} \sup _{s \in[0, t]}\left|\dot{f}_{h}(s)\right|\right\}
\end{aligned}
$$

The last inequality implies that the estimate $\hat{f}_{h}$ obtained by observer (4.17), (4.18) satisfies Assumption 8; therefore, Theorem 6 can be used to guarantee the overall stability of the teleoperator system.

\subsection{Experimental Results}

In this section, examples of experimental evaluation of the teleoperator system with the proposed projection-based FR algorithms are presented. In the experiments described below, the teleoperator system addressed is built according to positionerror based scheme described by equations (3.1), (3.2), (3.3), (3.4), (3.11), (3.12) with control law (3.5), (3.7) - (3.9). This is the same scheme as the one used during the experiments presented in section 3.9 ; all the parameters are also equal to the parameters given in section 3.9. Additionally, the teleoperator system under consideration is equipped with projection-based force reflection algorithm of the form (4.1), (4.2), where the estimate of the human torque is provided by high-gain torque observer described by (4.17), (4.18), with gain $\gamma=20$.

In general, the experiments performed clearly indicate that the proposed forcereflection algorithms substantially improve the admissible force-reflecting gain without loosing the overall stability of the teleoperator system. This improvement is achieved in the case of small (negligible) communication delays as well as in the case of significantly large irregular communication delays; it is worth noting, however, that in the latter case the improvement is somewhat more dramatic. The results of the experiments for negligible communication delays for different values 
of the weighting coefficient $\alpha$ are shown in figures 4.1-4.5; in these figures the force reflecting gain is equal to 4000 . In particular, figure 4.1 shows the position and force response of the teleoperator system for $\alpha=1$, which corresponds to the direct force reflection (this is because the weight of the projection-based term is $1-\alpha=0$ ). The resulting response is clearly unstable, which is in good accordance with the experimental data for direct force reflection algorithms presented in section 3.9. On the other hand, figure 4.2 shows the analogous response of the system with $\alpha=0.5$. (i.e., the weights of the direct force reflection and projection-based force reflection are equal); one can see that the response is perfectly stable. Indeed, since the operator releases the master immediately after returning back to origin, the corresponding projection of the reflected force onto the estimated vector of the human force becomes (approximately) zero, and so does the projection based force reflection term, which results in improved stability. The natural question, of course, is how does this type of force reflection affect the transparency of the system; in other words, what would the human operator feel if she/he does not release the master. The answer to this question can be illustrated by figure 4.3 , where, instead of releasing the master, the human operator tries to hold it firmly after returning to the origin. One can see that, in this case, the force reflected to the motors of the master actually follows quite closely the contact force generated on the slave side. The small difference between these force responses can be attributed to the fact that the estimate of the human force is obtained using a high-gain observer that, besides having its own dynamics, is built based on the model which probably has some discrepancy with the actual dynamics of the robot. The analogous results for $\alpha=0.25$ is presented in figures $4.4,4.5$.

In the next set of the experiments, the communication delays in both directions are set to be normally distributed random variables $\left(\tau(\cdot) \sim \mathbb{N}\left(T_{a v}, \sigma^{2}\right)\right)$ with mean 
$T_{a v}=1 \mathrm{sec}$, and standard deviation $\sigma=0.02 \mathrm{sec}$. Thus, the average RTT delay is equal to 2 sec. As shown in section 3.9, in this case the admissible FR gain (i.e., the maximal value of $\mathrm{FR}$ gain such that the system remains stable) for the system with direct force reflection $(\alpha=1)$ lies somewhere between 1000 and 1500 . Examples of the experimental results for the system with projection-based force reflection algorithm with FR Gain $=3000$ and $\alpha=0.3$ are shown in figures 4.6, 4.7. In particular, figure 4.6 represents the experiments where the human operator releases the master immediately after returning back to origin. One can see that the systen quickly stabilizes. On the other hand, the transparency properties of the system is illustrated by figure 4.7 , where the human operator holds firmly the master after returning to the origin. Again, one can see that in this case, the force reflected to the hand of the human operator follows closely the actual contact force generated on the slave side, thus confirming that there is virtually no transparency loss.

Overall, the experimental data indicate that the use of projection-based force reflection algorithms in bilateral teleoperation, particularly in the presence of communication delays, leads to drastic improvement in admissible force reflection gain without loosing the stability of the overall system. 

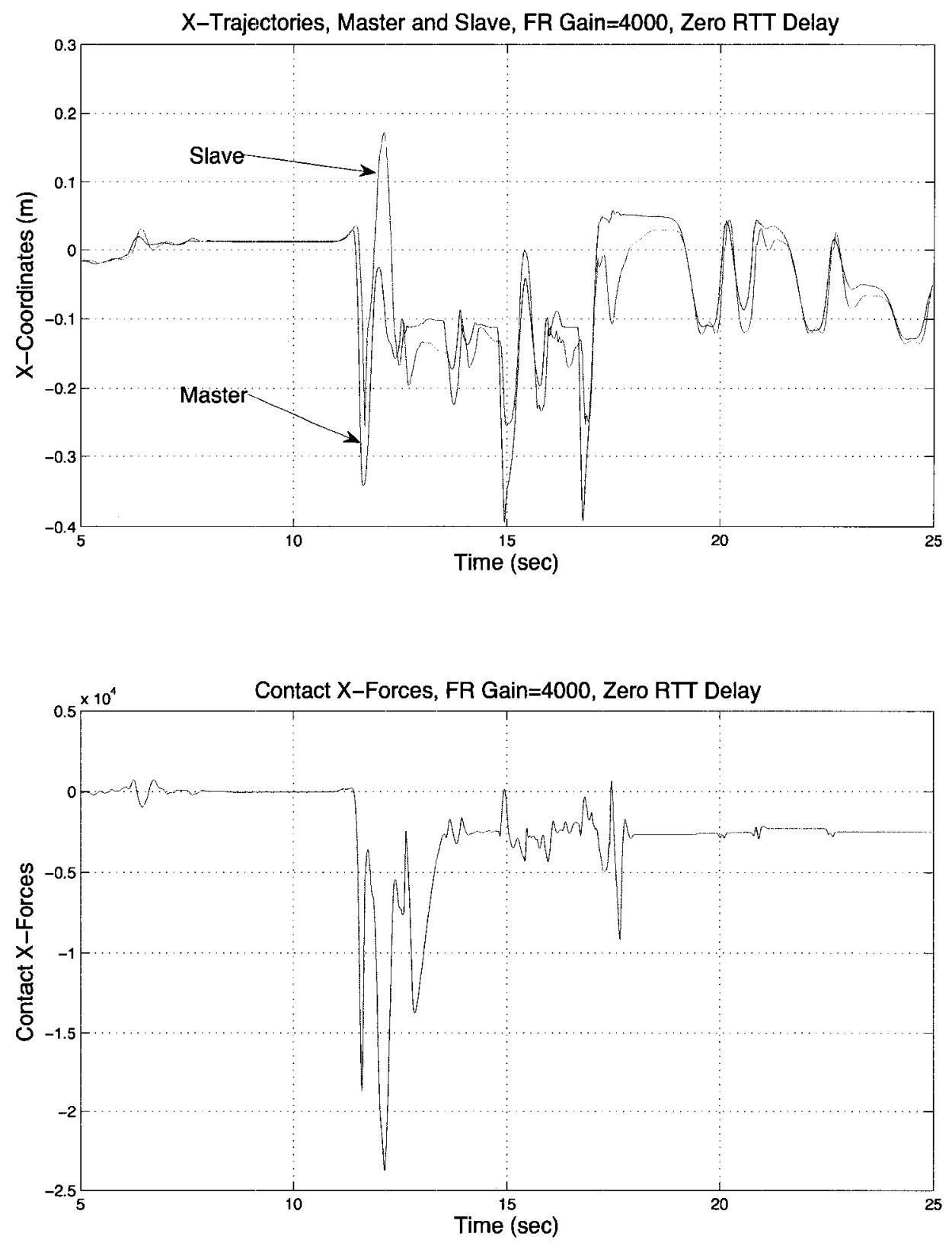

Figure 4.1: Teleoperator system response, Delay $\approx 0, \mathrm{FR}$ gain $=4000, \alpha=1$ (i.e., direct force reflection), free motion: $\mathrm{X}$-trajectories, master and slave (top); Contact X-Forces (bottom) 

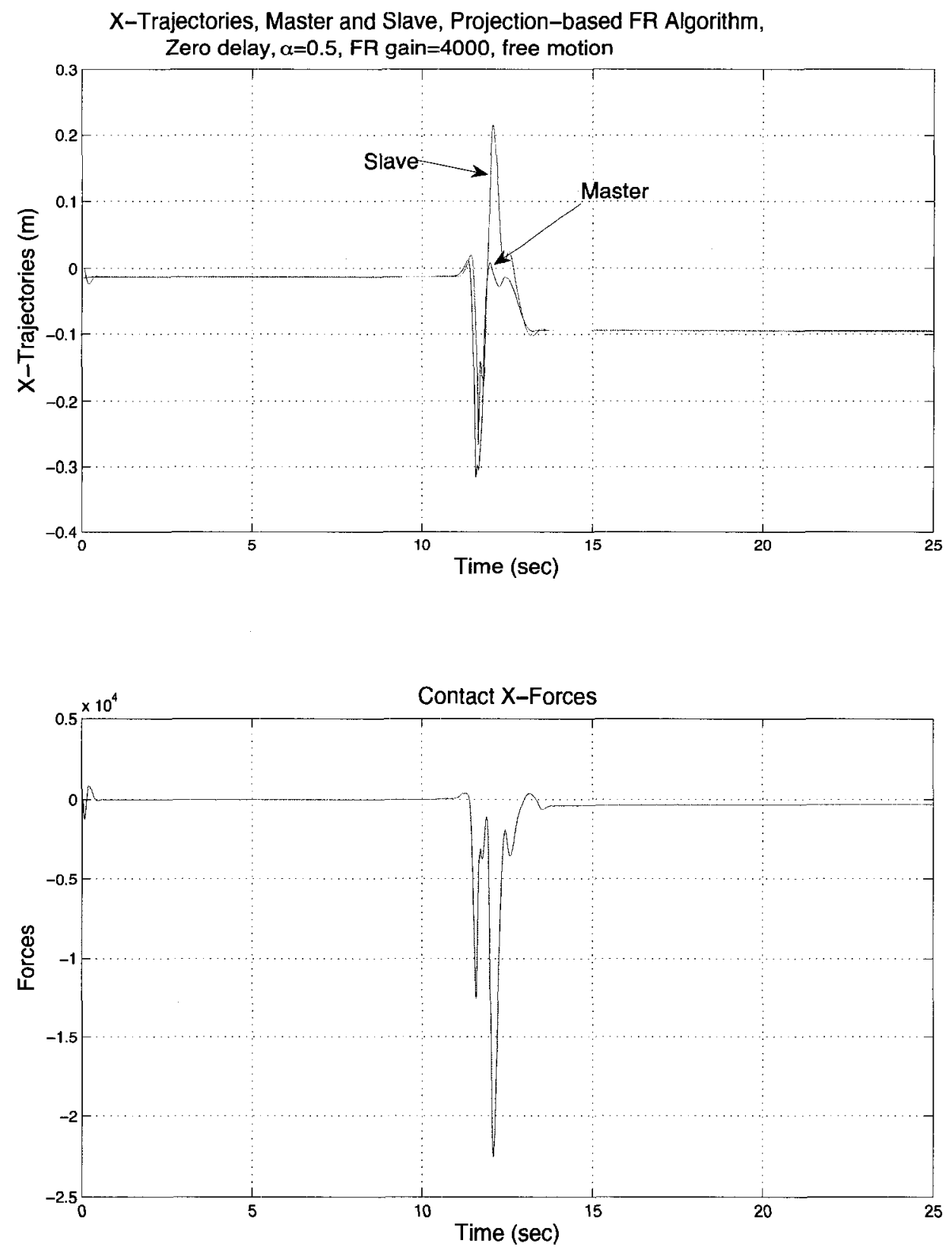

Figure 4.2: Teleoperator system response, Delay $\approx 0, \mathrm{FR}$ gain $=4000, \alpha=0.5$, free motion: X-trajectories, master and slave (top); Contact X-Forces (bottom) 

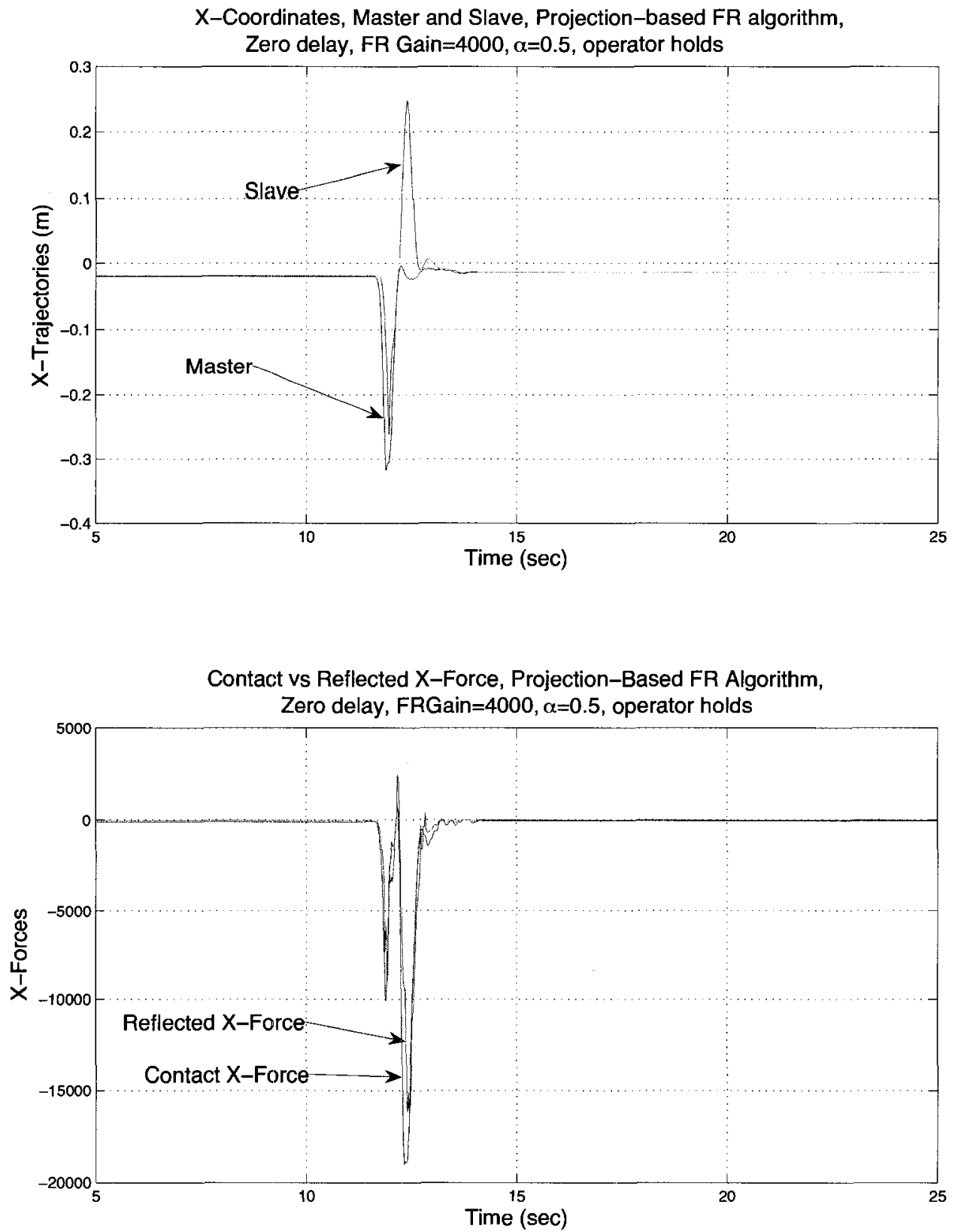

Figure 4.3: Teleoperator system response, Delay $\approx 0, \mathrm{FR}$ gain $=4000, \alpha=0.5$, operator holds: X-trajectories, master and slave (top); Contact X-Forces vs. Reflected X-Forces(bottom) 

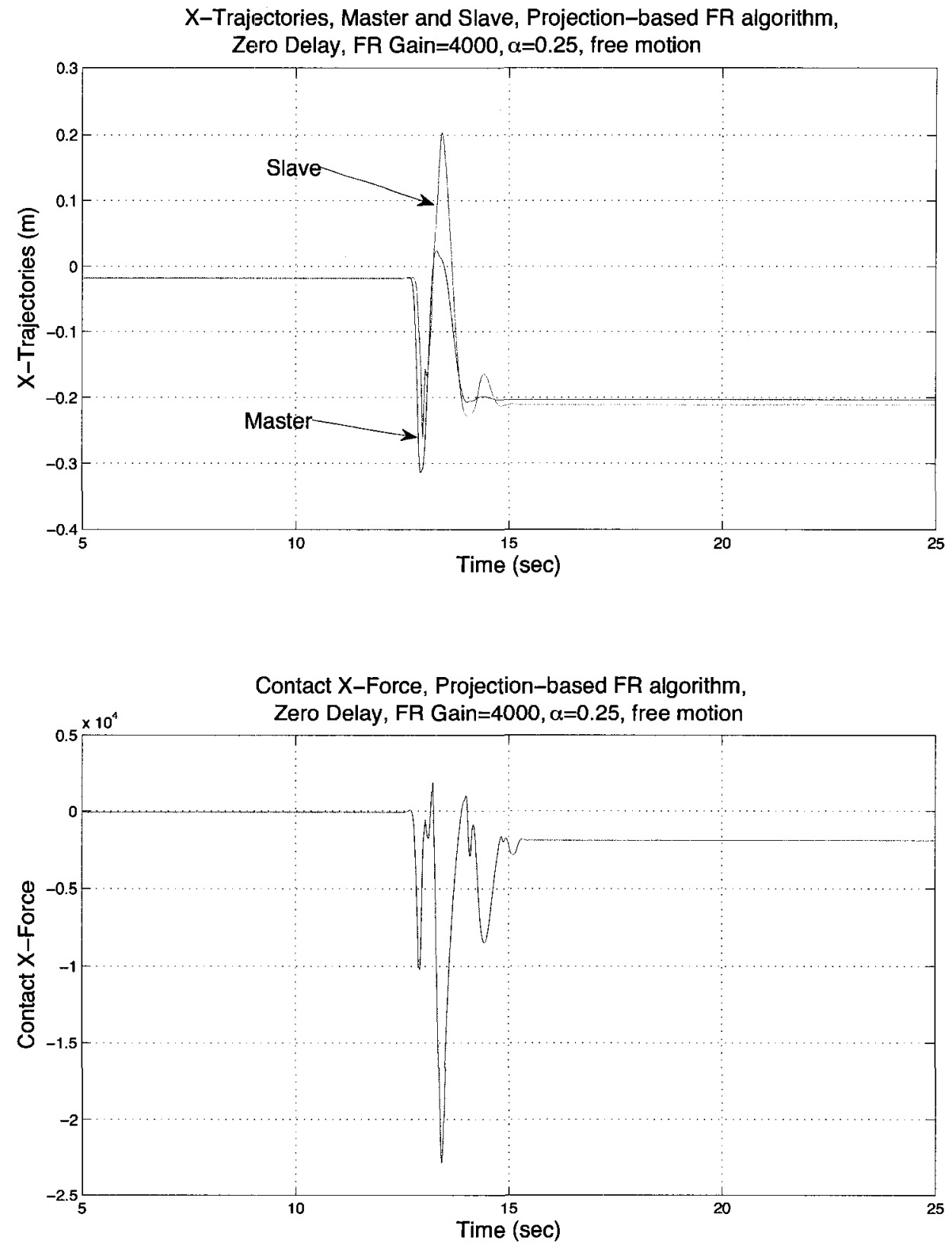

Figure 4.4: Teleoperator system response, Delay $\approx 0, \mathrm{FR}$ gain $=4000, \alpha=0.25$, free motion: X-trajectories, master and slave (top); Contact X-Forces (bottom) 

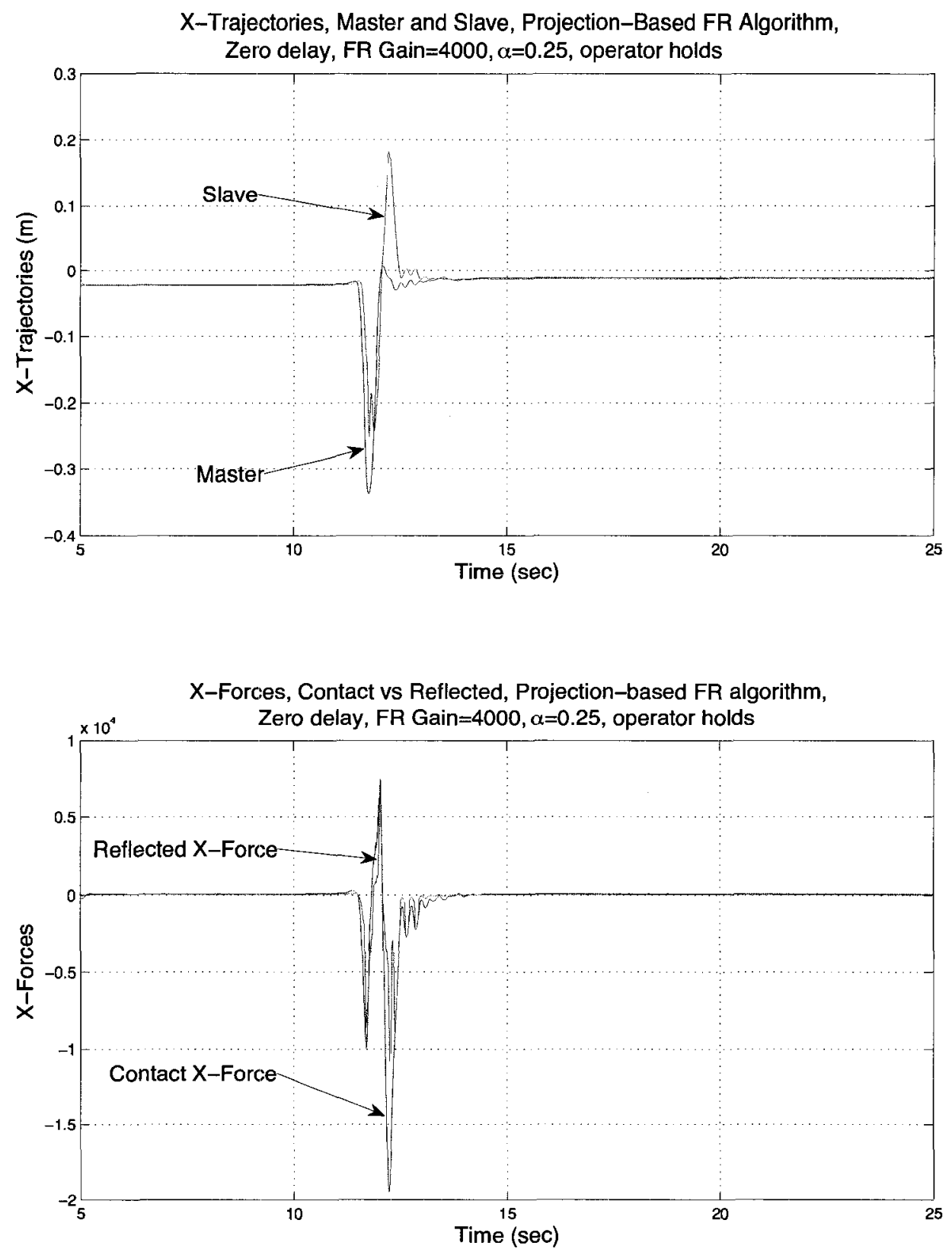

Figure 4.5: Teleoperator system response, Delay $\approx 0$, FR gain $=4000, \alpha=0.25$, operator holds: X-trajectories, master and slave (top); Contact X-Forces vs. Reflected X-Forces(bottom) 

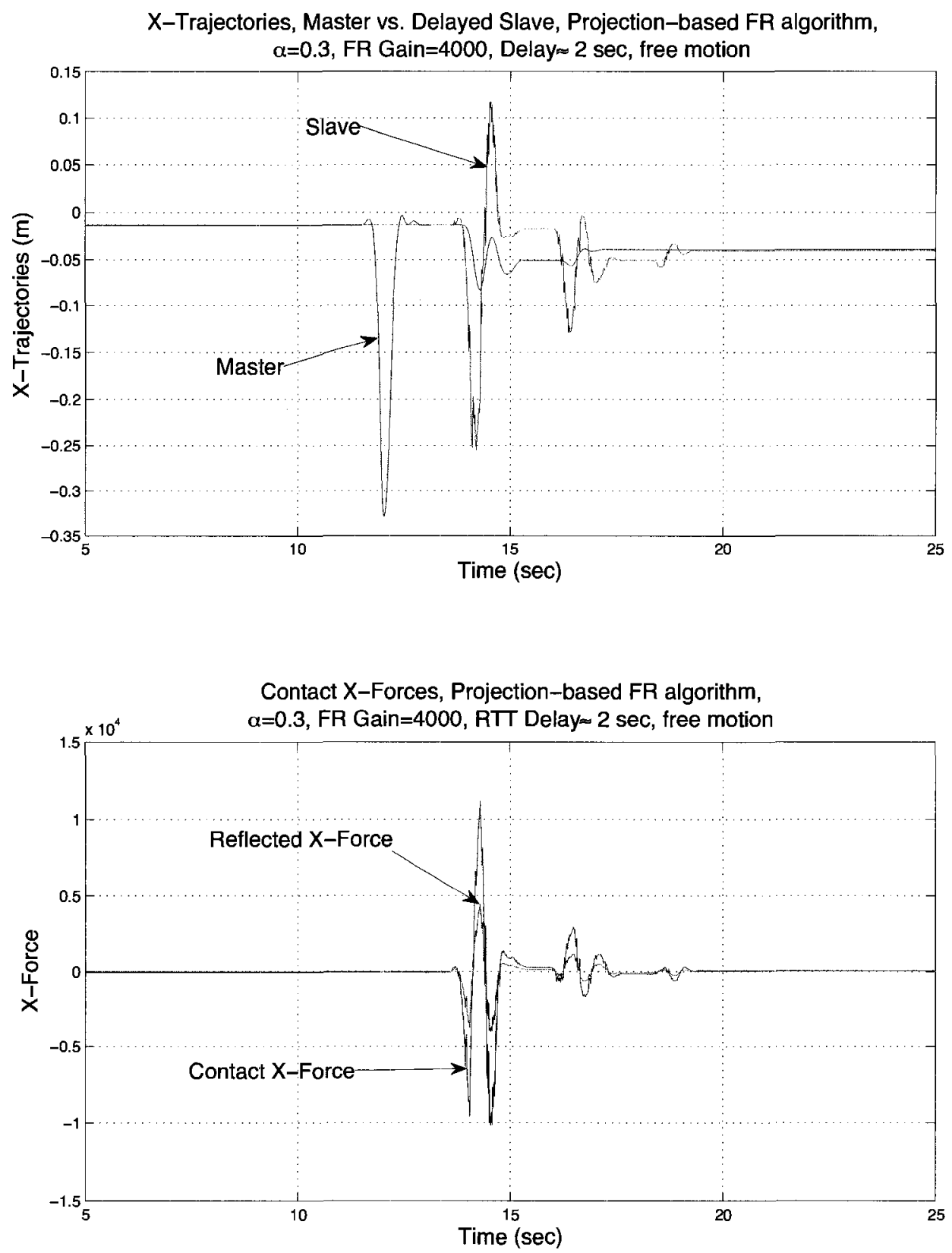

Figure 4.6: Teleoperator system response, Delay $\approx 2$ sec, FR gain $=4000, \alpha=0.3$, free motion: X-trajectories, master and slave (top); Contact X-Forces (bottom) 

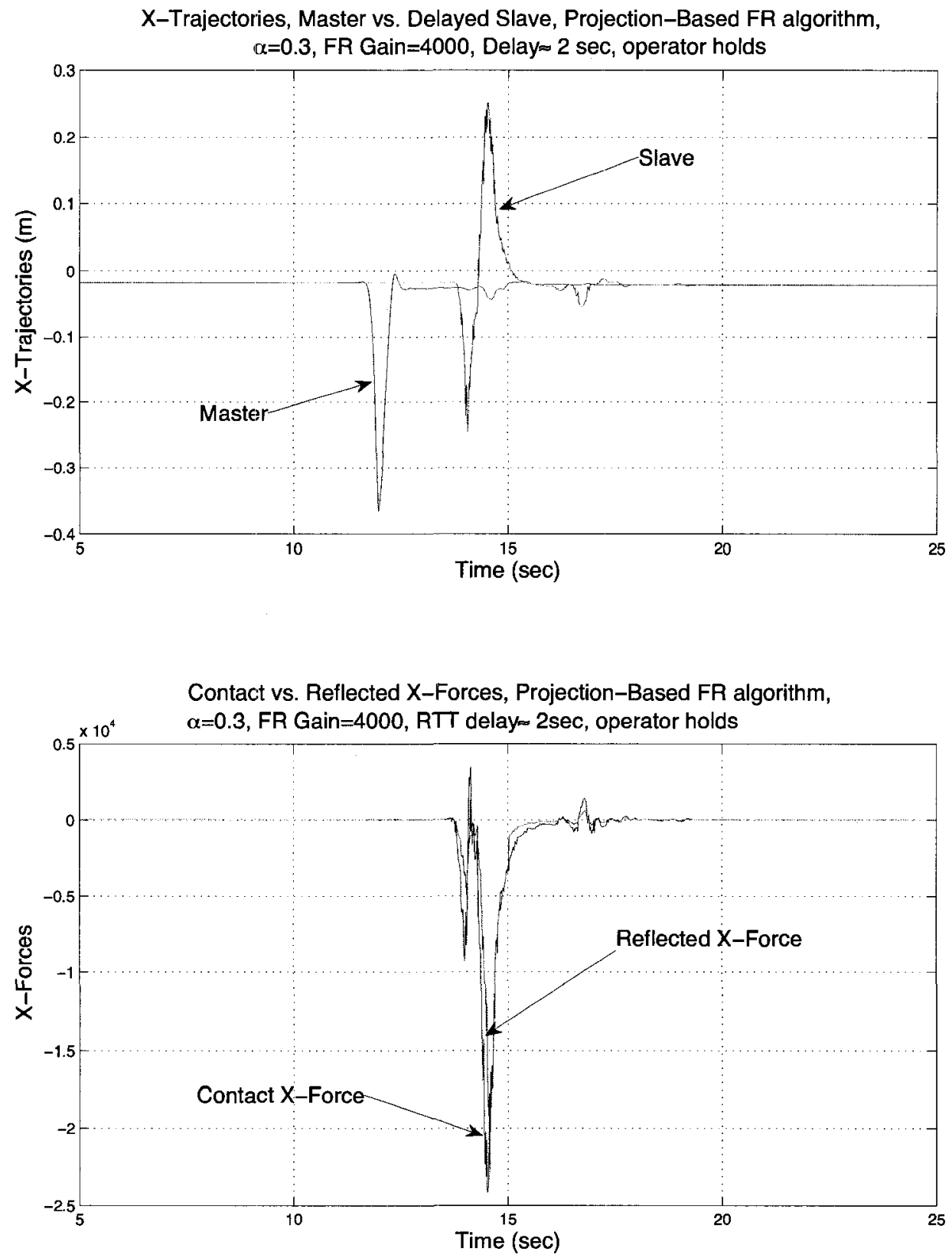

Figure 4.7: Teleoperator system response, Delay $\approx 2$ sec, FR gain $=4000, \alpha=0.3$, operator holds: X-trajectories, master and slave (top); Contact X-Forces vs. Reflected X-Forces(bottom) 


\subsection{Conclusions}

Traditionally, the small-gain approach is considered to be not particularly suitable for teleoperation tasks, since it " ... would result in conservative design criteria (leading to poor transparency)..." [15]. Indeed, for force reflecting teleoperator systems, the direct application of the small-gain approach results in significant constraints on the force reflection gain, as well as generally requires high damping/stiffness of the master manipulator. In this chapter, we show that, using the projection-based force reflection algorithms, the constraints on subsystem's gains can be effectively removed. In particular, this implies that the stability can be achieved for arbitrarily high force reflection gain and arbitrarily low damping/stiffness of the master manipulator. Essentially, the proposed approach reduces the design of stable force reflecting teleoperator system with communication delay to the design of two stable subsystems. It is worth noting that this is achieved by utilizing certain fundamental characteristics of human force sensing and without paying the price in terms of transparency deterioration. Overall, the use of projection-based force reflection algorithms may significantly improve the applicability of the small-gain methods to the design of the force-reflecting teleoperator systems, particularly in the presence of network induced communication constraints. 


\section{Chapter 5}

\section{Model-Based Teleoperation with Communication Constraints}

\subsection{Introduction}

In the previous chapters, the problem of stable and transparent force reflecting teleoperation in the presence of network induced communication constraints was addressed, and some new solutions were proposed. In general, however, even in the case of perfect stability and perfect (delayed) transparency (although generally these properties can not be achieved simultaneously), the existence of significant communication delays inevitably leads to loss of the sense of causality for the human operator. For example, it is confirmed experimentally that in telesurgery systems, even in the situation where the human surgeon is exposed to a visual feedback and no force feedback applied, the surgeon effectively loses his/her ability to operate when communication delay exceeds $500 \mathrm{~ms}$ [3]. Moreover, in presence of the haptic feedback, the human operator tolerance to communication delay decreases significantly. According to $[73 ; 74]$, in this case the admissible upper bound for round-trip 
time (RTT) delay is up to $40 \mathrm{~ms}$ for abrupt movements and up to $160 \mathrm{~ms}$ for gentle movement. This implies that even a perfectly designed force reflecting teleoperator system may become increasingly hard to control if the underlying communication channel does not deliver the data with reasonably small time delay and jitter. Thus, although the direct force feedback usually improves the ability of the operator to perform the task, the implementation of such a feedback may be extremely challenging, or may require solutions whose complexity and cost are beyond acceptable limits.

One alternative approach to the delayed force feedback is to create a virtual environment (VE) on the master side that duplicates the essential features of the remote slave+environment interconnection. In this case, the human operator may use the local (direct, in the terminology of [75]) force feedback that is generated by the virtual "slave+environment" model in order to obtain a certain "feeling" of the process. Such a local force feedback is not subject to communication delay, which restores the sense of causality for the human operator and does not have the destabilization effect of the delayed force feedback. Such a "virtual" force feedback, however, is generally prone to modelling errors. The local ("direct" [75]) force feedback can also be combined with the delayed force feedback from the remote environment ("indirect" force feedback) to form the dual force feedback. Other references related to this topic include (but by no means limited to) $[76 ; 77 ; 78 ; 79$; $80 ; 81]$, some survey is also presented in [82, Section 10.5].

In this chapter, we first present a model-based approach to control of nonlinear systems in presence of significant network-induced communication constraints, and then apply (and in some aspects, further develop) this approach to design of virtual reality enhanced bilateral teleoperator systems. In general, the approach presented here utilizes some ideas from nonlinear sampled-data system design $[53 ; 83]$ and, in 
particular, its extension to multi-rate sampled data systems and model-based control over networks presented in $[84 ; 85]$. Essentially, it is based on the assumption that a partially known set of approximate discrete-time models of the remote process is available for the designer. Based on this assumption, control algorithms and communication protocols are presented that guarantee stability of the overall system under mild assumptions imposed on communication process. The structure of this chapter is as follows. In Section 5.2, the model-based approach is presented for a general case of nonlinear systems controlled over communication networks. In Section 5.3 , the approach is further developed for the case of network-based bilateral teleoperators. Simulation example is presented in Section 5.4, and finally, concluding remarks are given in Section 5.5.

\subsection{Model-Based Approach to Nonlinear Networked Control Systems}

In recent years, the problem of control of dynamical systems over communication networks attracts considerable research interest (see for example $[86 ; 87 ; 88 ; 89 ; 90$; 91; 92], among many other publications). In networked control systems (NCS), the controller and the plant exchange the information over a network communication channel. The presence of a network in the control loop may result in significant communication constraints imposed on the control process. These constraints may include irregularity of transfer intervals, existence of communication delays, and possibility of packet losses, among others. In particular, traditional control applications usually require data exchange between the plant and the controller to be performed at a fixed sampling rate, therefore, the transfer interval (the interval between two consecutive transmissions) must be constant and known. This assumption is not 
normally satisfied even for many types of local area networks (LANs), such as the Ethernet with CSMA/CD, where the transfer interval is a random variable whose characteristics essentially depend on the network load. Also, in traditional data networks the information is normally transmitted in relatively large packets, for example, the length of data field in the Ethernet frame is limited from below by 46 bytes. On the contrary, control applications send data in short messages, therefore, in this case the Ethernet frames have to be "stuffed", which results in inefficient utilization of the networks bandwidth. Furthermore, if a control process is performed over a wide area network (WAN), additional problems arise. Occurrence of congestion, for example, may result in significant communication delay and data packet losses. Finally, real-time control applications normally require clock synchronization between the plant and the controller which may not be easy to achieve even in LANs such as the Ethernet [93]. Because of these specific constraints, direct application of many traditional control techniques to NCSs may be impossible, or may lead to performance degradation and/or instability.

Recently, several specific control techniques have been developed that deal with communication constraints imposed by networks. One such technique is modelbased control [94; 95]. In model-based networked control systems, a model of the plant is included into the controller node, and the control action is calculated based on the current state of the model rather than the actual state of the plant. On the other hand, the model state is updated from time to time based on measurement of the actual state of the plant. Essentially, the plant is controlled in open-loop when the network is not available for transmission. This strategy generally leads to significantly lower communication bandwidth requirements, since the update of the model's state can usually be performed at a much lower rate comparing to the one with which the control action is generated. Thus, the model-based approach utilizes 
a trade-off between two control strategies, the closed-loop control which allows to guarantee good stability/performance characteristics but requires frequent data exchange between the controller and the plant with strong real-time requirements, and the open-loop control which requires very little communication (no communication at all in the "ultimate" case), however, lacks stability/performance guarantees.

In this Section, we address a problem of model-based sampled-data control of a nonlinear system where the communication between the plant and the controller are subject to network-induced constraints. Comparing to the previous results on model-based NCS (for example, in [94; 95]), our work has several distinct features. First, in $[94 ; 95]$, it is assumed that a network is located in the feedback (measurement) path of the control loop, however, no communication constraints are imposed on the feedforward (control) path. This assumption significantly simplifies the problem, however, it does not reflect the most common real-life situation where the plant and the controller are located at different nodes and, therefore, both feedforward and feedback signals are transmitted over the network. We address the problem in a more general setting where both the feedforward and the feedback paths are subject to network induced constraints. In particular, we assume that the transfer interval (i.e., the interval between two consecutive transmissions) in both feedforward and feedback paths is time varying and unknown. Further, communication delays are assumed to be negligible in [94; 95], with the exception of Theorem 3 in [94], where the communication delay is assumed to be constant, known, and limited to the feedback path of the networked control system. Also, no information is assumed in $[94 ; 95]$ to be lost during communication process. However, transport delays are unavoidable when communication is performed over WANs due to packet queuing, and congestions may lead to unpredictable variations of transport delay as well as packet dropouts. Therefore, the control problem is addressed in our work 
under the assumption that the time delays in both the channels are time-varying and unknown, and some packets may be lost. Also, since the clock synchronization may pose a significant problem even in LANs, we address the control problem assuming that the clock synchronization between the controller and the plant nodes is not possible. To deal with the control problem under all these assumptions, we propose a new protocol for communication between the controller and the plant nodes. Finally, most of the previous results (with an exception of [95, Theorem 12]) address linear NCSs. The essential feature of a nonlinear case is that the exact discrete-time model of a continuous-time nonlinear system is usually not possible to obtain; instead, the designer rather deals with a set of approximate models of the plant [52]. We utilize the multi-rate approach to controller design for nonlinear sampled-data systems [84], although the results are presented in this paper for the case of dynamic controller which is not addressed in [84]. We formulate and prove stability result for the proposed NCS, and show that performance of non-networked control system can be recovered in model-based NCS; more precisely, the decaying estimate for the trajectories of NCS can be achieved arbitrarily close to the one valid for non-networked system. Additionally, it is demonstrated that the maximum allowable transfer interval (MATI) in the proposed model-based NCS can be assigned arbitrarily by an appropriate choice of the design parameters. The results presented in this section form a basis for our development of a scheme for virtual environment enhanced bilateral teleoperation over networks presented in section 5.3.

\subsubsection{Sampled-Data Stabilization of Nonlinear Systems}

Consider a nonlinear time-varying continuous-time system which is connected to a digital network through discrete-analog $(\mathrm{D} / \mathrm{A})$ and analog-discrete $(\mathrm{A} / \mathrm{D})$ converters. For a fixed sampling period $T>0$, such a system is described by its exact 
discrete-time model of the form

$$
x(i+1)=F^{+}(x(i), u(i), i),
$$

where $x \in \mathbb{R}^{n}$ is a state, $u \in \mathbb{R}^{m}$ is a (discrete-time) control input, and $i \in \mathbb{Z}^{+}=$ $\{0,1, \ldots\}$. In most situations, the exact discrete-time model (5.1) is not possible to obtain, since it requires exact integration of the original nonlinear differential equation. Instead, the designer rather deals with a set of approximate discrete-time models of the form

$$
\hat{x}(i+1)=f_{h}^{+}(\hat{x}(i), u(i), i),
$$

where the right-hand side depends on a modelling parameter $h>0$. Generally, the modelling parameter is any parameter which may be used to enhance the accuracy of the approximate model; the closer $h$ to 0 , the better accuracy is achieved. A common example of the modelling parameter is the integration step of the numerical approximation scheme. Standard sufficient condition for stabilization of the system (5.1) based on its approximate models (5.2) include [52] existence of a set of control laws that asymptotically stabilize the approximate models (5.2) and multi-step consistency of the approximation scheme. Versions of these properties used in this paper are as follows.

Assumption 9. (Lyapunov certificates for asymptotic stabilization of a set of approximate models) There exist a set of Lyapunov functions $V_{h}: \mathbb{R}^{n} \times \mathbb{R}^{p} \rightarrow \mathbb{R}^{+}$ and a set of control algorithms

$$
\begin{aligned}
z(i+1) & =Z_{h}(z(i), x(i), i) \\
u(i) & =U_{h}(z(i), x(i), i),
\end{aligned}
$$

both parameterized by modelling parameter $h \in\left(0, h^{*}\right], h^{*}>0$, with the following properties: $V_{h}$ is continuous in first argument uniformly for $h \in\left(0, h^{*}\right]$ and satisfying

$$
\alpha_{1}(\max \{|x|,|z|\}) \leq V_{h}(x, z) \leq \alpha_{2}(\max \{|x|,|z|\})
$$


uniformly for $h \in\left(0, h^{*}\right]$, where $\alpha_{1}, \alpha_{2} \in \mathcal{K}_{\infty} ; U_{h}(z, x, i)$ is bounded on an arbitrary bounded set of $z, x$, uniformly for all $h \in\left(0, h^{*}\right]$ and $i \in \mathbb{Z}^{+}$; and the inequality

$$
\Delta V_{h}(x, z, i):=V_{h}\left(f_{h}^{+}\left(x, U_{h}(z, x, i), i\right), Z_{h}(z, x, i)\right)-V_{h}(x, z) \leq-\alpha_{3}\left(V_{h}(x, z)\right)
$$

holds for all $i \in \mathbb{Z}^{+}$and all $h \in\left(0, h^{*}\right]$.

Assumption 10. (Multi-Step Time-Uniform Consistency of the Approximation Scheme) Given $\Delta_{x}, \Delta_{u} \geq 0$, there exists a function $\rho_{\Delta_{x}, \Delta_{u}}: \mathbb{N} \times \mathbb{R}^{+} \rightarrow$ $\mathbb{R}^{+}$such that $\rho_{\Delta_{x}, \Delta_{u}}(i, h)$ is a $\mathcal{K}$-class function in $h>0$ and strictly increasing in $i \in \mathbb{N}$, such that the following holds. For any $x_{0} \in \mathbb{R}^{n},\left|x_{0}\right| \leq \Delta_{x}$, any $u_{0}, u_{1}, \ldots, u_{j} \in \mathbb{R}^{m}, j \in \mathbb{Z}^{+},\left|u_{i}\right| \leq \Delta_{u}, i=0,1, \ldots, j$, and any $i_{0} \in \mathbb{Z}^{+}$such that $F^{+}\left(\ldots F^{+}\left(F^{+}\left(x_{0}, u_{0}, i_{0}\right), u_{1}, i_{0}+1\right), \ldots, u_{j}, i_{0}+j\right)$ is well-defined, the following holds

$$
\begin{gathered}
\mid F^{+}\left(\ldots F^{+}\left(F^{+}\left(x_{0}, u_{0}, i_{0}\right), u_{1}, i_{0}+1\right) \ldots, u_{j}, i_{0}+j\right) \\
-f_{h}^{+}\left(\ldots f_{h}^{+}\left(f_{h}^{+}\left(x_{0}, u_{0}, i_{0}\right), u_{1}, i_{0}+1\right) \ldots, u_{j}, i_{0}+j\right) \mid \\
\leq \rho_{\Delta_{x}, \Delta_{u}}(j, h) . \bullet
\end{gathered}
$$

Under Assumptions 9 and 10, the closed-loop system (5.1), (5.3) is semi-globally practically asymptotically stable if $h>0$ is sufficiently small. For more advanced and detailed results of this type, the reader is referred to [52; 53]. A partial extension of these results to the case of multi-rate (model-based) stabilization is given in [84].

In this paper, we address a problem of sampled-data stabilization of a nonlinear system (5.1) based on its approximate model (5.2) in the situation where the plant and the controller communicate over a network. More precisely, we assume that the plant and the controller are located in separate nodes, so that all communications between them in both directions are subject to network-induced constraints. These constraints include irregularity of transfer intervals, existence of (time-varying and unknown) communication delays, and the possibility of packet dropouts. Formally, let $\mathbb{J}_{0} \subset \mathbb{Z}^{+}$be a communication sequence for the plant (5.1). For each $i \in \mathbb{J}_{0}$, the 
plant state $x(i)$ is measured and the corresponding data are sent over the network to the controller node $(\mathrm{CN})$. Also, for each $i \in \mathbb{J}_{0}$, let $d(i) \in \mathbb{Z}^{+}$be the corresponding total round-trip time (RTT) delay. This means that, if a state measurement is performed at $i$-th sampling instant, $i \in \mathbb{J}_{0}$, the corresponding control action is available at the plant node (PN) starting from $i+d(i)$-th sampling instant. At this point, no restrictions are applied on $\mathbb{J}_{0}$ and $d(\cdot)$; in particular, it is possible that $d(i)=+\infty$ for some $i \in \mathbb{J}_{0}$, which corresponds to the case of packet dropout.

To deal with the stabilization problem under these constraints, we utilize a model-based approach to controller design. The model-based controller is a controller of the form (5.3) augmented by an approximate model (5.2), as follows

$$
\begin{aligned}
\hat{x}(i+1) & =f_{h}^{+}(\hat{x}(i), u(i), i), \\
z(i+1) & =Z_{h}(z(i), \hat{x}(i), i), \\
u(i) & =U_{h}(z(i), \hat{x}(i), i) .
\end{aligned}
$$

Here, as before, $\hat{x}$ is a state of the model, $z$ is a controller state, and $h>0$ is a modelling parameter. The main idea behind the model-based controller (5.5)-(5.7) is that the control action $u$ is calculated based on the model state $\hat{x}$ rather than the actual plant state $x$. The state $\hat{x}$ of the model, however, is updated based on the actual measurements of the plant state $x(i), i \in \mathbb{J}_{0}$. In the presence of communication constraints, this approach has an advantage over the traditional (non-model-based) one, since it generally requires lower bandwidth for communication; indeed, the model state $\hat{x}$ can be updated at a significantly lower rate comparing to the control action generation.

Remark 11. Multi-step consistency property can be guaranteed, for example, in terms of one-step consistency of the approximation scheme and continuity of the set of approximate models uniform in $h$ as $h \rightarrow 0+$, as follows. 
Proposition 5. Suppose for any given $\Delta_{x}, \Delta_{u} \geq 0$, there exist $\rho(\cdot) \in \mathcal{K}, \lambda_{x} \in \mathcal{K}$, and $h^{*}>0$, such that the following two properties hold:

i) One-Step Time-Uniform Consistency of the Approximation Scheme: for any $x \in \bar{B}\left(\Delta_{x}\right)$, any $u \in \bar{B}\left(\Delta_{u}\right)$, and any $i \in\{0,1, \ldots\}$,

$$
\left|F^{+}(x, u, i)-f_{h}^{+}(x, u, i)\right| \leq \rho(h)
$$

ii) Uniform Continuity in $x$ of Approximate Models: for any $x_{1}, x_{2} \in \bar{B}\left(\Delta_{x}\right)$, $u \in \bar{B}\left(\Delta_{u}\right), i \geq 0$, and $h \in\left(0, h^{*}\right]$,

$$
\left|f_{h}^{+}\left(x_{1}, u, i\right)-f_{h}^{+}\left(x_{2}, u, i\right)\right| \leq \lambda_{x}\left(\left|x_{1}-x_{2}\right|\right) .
$$

Then the approximation scheme is multi-step consistent.

\subsubsection{Protocol for Model-Based NCS}

This section describes a communication protocol for model-based NCS (5.1), (5.5)(5.7). First, we assume that $\mathrm{PN}$ is equipped with an input buffer $\mathrm{U}_{p}=\{u(i), u(i+1), \ldots\}$, which contains, for each time instant $i \in \mathbb{Z}^{+}$, a sequence of "future" control signals $u(i), u(i+1)$, etc. On the other hand, $\mathrm{CN}$ is equipped with four buffers $\mathbf{U}_{c}, \mathbf{U}_{c}^{t e m p}$, $\mathbf{Z}_{c}, \mathbf{Z}_{c}^{\text {temp }}$. We also assume that an estimate for admissible upper bound of RTT $\hat{d}$ is maintained at PN. We do not specify how this estimate is maintained exactly; it may be a result of network measurements, execution of certain algorithms, or, in the simplest case, an appropriately chosen positive constant. Additionally, the communication protocol utilizes the following set of variables: $n_{p} \in\{0,1\}, \tau_{p} \in \mathbb{Z}^{+}$, $j_{p}^{*} \in \mathbb{Z}^{+}$at PN, and $n_{c} \in\{0,1\}, \tau_{c} \in \mathbb{Z}^{+}, j_{c}^{*}, j_{c t}^{*} \in \mathbb{Z}^{+}$at CN. Formally, the protocol can be described as follows.

\section{Initialization:}

$$
\mathbf{U}_{p}=\mathbf{U}_{c}=\mathbf{U}_{c}^{t e m p} \leftarrow \mathcal{U}^{0}:=\left\{u^{0}(0), u^{0}(1), \ldots\right\}
$$




$$
\begin{aligned}
& \mathbf{Z}_{c}=\mathbf{Z}_{c}^{\text {temp }} \leftarrow\{0,0, \ldots\} \\
& n_{p}=n_{c} \leftarrow 0, \tau_{p}=\tau_{c} \leftarrow 0, j_{p}^{*}=j_{c}^{*}=j_{c t}^{*} \leftarrow 0
\end{aligned}
$$

\section{Plant Node:}

for $i \in \mathbb{Z}^{+}, i \geq j_{0}$

control: $u(i) \leftarrow \mathbf{U}_{p}(i)$ is applied to the input of (5.1);

if $i \in \mathbb{J}_{0}$ (i.e., $x=x(i)$ is measured)

$$
\begin{aligned}
& \text { obtain } \hat{d} \leftarrow \hat{d}(i) \\
& \text { set } \tau_{p} \leftarrow i-j_{p}^{*} \\
& \text { send } \mathrm{D}_{p \rightarrow c}:=\left\{x ; \hat{d} ; \tau_{p} ; n_{p}\right\} \text { to } \mathrm{CN}
\end{aligned}
$$

if the new data $\mathbf{D}_{c \rightarrow p}:=\left\{\mathbf{U}_{c}^{t e m p}(i), i \geq j_{c t}^{*}+\hat{d} ; \tau_{c}\right\}$ arrived from CN

$$
\begin{aligned}
& \text { if } \tau_{c}=\tau_{p} \text { and } \hat{d} \geq d\left(j_{p}^{*}+\tau_{p}\right) \\
& \qquad \mathbf{U}_{p}(i) \leftarrow \mathbf{U}_{c}^{t e m p}(i) \text { for } i \geq j_{p}^{*}+\tau_{p}+\hat{d} \\
& \quad j_{p}^{*} \leftarrow j_{p}^{*}+\tau_{p}, n_{p} \leftarrow n_{p}+1(\bmod 2) ; \\
& \text { otherwise }\left(\tau_{c} \neq \tau_{p} \text { or } \hat{d}<d\left(j_{p}^{*}+\tau_{p}\right)\right) \\
& \text { discard } \mathbf{D}_{c \rightarrow p}
\end{aligned}
$$

\section{Controller Node:}

if the new data $\mathbf{D}_{p \rightarrow c}:=\left\{x ; \hat{d} ; \tau_{p} ; n_{p}\right\}$ is received if $n_{c} \neq n_{p}$

$$
\mathbf{Z}_{c} \leftarrow \mathbf{Z}_{c}^{\text {temp }}, \mathbf{U}_{c} \leftarrow \mathbf{U}_{c}^{t e m p}, j_{c}^{*} \leftarrow j_{c t}^{*}, n_{c} \leftarrow n_{p} ;
$$

set $j_{c t}^{*}=j_{c}^{*}+\tau_{p}$

calculate $\hat{x}\left(j_{c t}^{*}+\hat{d}\right)$ as the solution of (5.5) with IC $\hat{x}_{h}\left(j_{c t}^{*}\right)=x$, and input

$$
u(i) \in \mathbf{U}_{c}
$$


calculate $\hat{x}(i), z(i), u(i)$ for $i \geq j_{c t}^{*}+\hat{d}$ as the solution of $(5.5)-(5.7)$ with $\operatorname{ICs} \hat{x}\left(j_{c t}^{*}+\hat{d}\right)$ and $z\left(j_{c t}^{*}+\hat{d}\right) \leftarrow \mathbf{Z}_{c}$

$$
\begin{aligned}
& \mathbf{U}_{c}^{t e m p}(i) \leftarrow \mathbf{U}_{c}(i) \text { for } i \in\left\{j_{c t}^{*}, \ldots, j_{c t}^{*}+\hat{d}\right\} \\
& \mathbf{Z}_{c}^{t e m p}(i) \leftarrow \mathbf{Z}_{c}(i) \text { for } i \in\left\{j_{c t}^{*}, \ldots, j_{c t}^{*}+\hat{d}\right\} \\
& \mathbf{U}_{c}^{t e m p}(i) \leftarrow u(i), \mathbf{Z}_{c}^{t e m p}(i) \leftarrow z(i) \text { for } i>j_{c t}^{*}+\hat{d} \\
& \tau_{c} \leftarrow \tau_{p}
\end{aligned}
$$

send $\mathbf{D}_{c \rightarrow p}:=\left\{\mathbf{U}_{c}^{t e m p}(i), i \geq j_{c t}^{*}+\hat{d} ; \tau_{c}\right\}$ to PN

The above protocol can be explained as follows. Initially, buffers $\mathbf{U}_{p}, \mathbf{U}_{c}, \mathbf{U}_{c}^{t e m p}$ contain a precalculated sequence of control signals $\mathcal{U}^{0}$. For each $i \in \mathbb{Z}^{+}$, the control signal $u(i)$ is retrieved from the buffer $\mathbf{U}_{p}$ and applied to the input of the plant (5.1). The content of the buffer $\mathrm{U}_{p}$ is updated according to the following scheme. For each transfer instant $i \in \mathbb{J}_{0}$, the plant state $x(i)$ is measured, and the measurement data (together with the current values of $\hat{d}, \tau_{p}$ and $n_{p}$, the purpose of all these is explained below) are sent over network to $\mathrm{CN}$. Upon receiving the data, $\mathrm{CN}$ updates the state of the plant's model according to the formula $\hat{x}(j):=x(j)$, and then calculates the sequence of control signals $u(i), i \geq j$, that corresponds to the solution of the closed loop "plant's model+controller" system (5.5)-(5.7). For simplicity, let us assume that these control signals are calculated for all future instants $i \geq j$; however, only a finite number of $u(i)$ is actually required (see Remark 15 below). This control sequence is then transmitted in a single packet to PN. Upon receiving the packet, the plant node extracts the control sequence and updates the buffer $\mathrm{U}_{p}$.

The parameters $\tau_{p}, \tau_{c}, n_{p}$, and $n_{c}$ are introduced to achieve coordination between $\mathrm{PN}$ and $\mathrm{CN}$, i.e., to guarantee that the control sequence applied to the plant's input is exactly the same as the one "known" by the controller. In particular, $\tau_{p}$ is defined as a number of sampling instants between the current state measurement and the 
last "successful" one. Upon receiving the data from PN, CN first identifies the time instant $j_{c t}^{*}$ when the measurement was performed, according to the formula $j_{c t}^{*}=j_{c}^{*}+\tau_{p}$, where $j_{c}^{*}$ is the time instant of the previous "successful" measurement. Next, CN calculates $\hat{x}\left(j_{c t}^{*}+\hat{d}\right)$ (which is an estimate for $x\left(j_{c t}^{*}+\hat{d}\right)$ ) as a solution of (5.5) with initial condition $\hat{x}_{h}\left(j_{c t}^{*}\right)=x$ under the control sequence $u(i), i=j, \ldots, j+\hat{d}(j)$; the latter is retrieved from the buffer $\mathbf{U}_{c}$. After that, CN calculates $\hat{x}(i), z(i), u(i)$ for $i \geq j_{c t}^{*}+\hat{d}$ as a solution of the closed-loop system (5.5)-(5.7) with initial conditions $\hat{x}\left(j_{c t}^{*}+\hat{d}\right)$ and $z\left(j_{c t}^{*}+\hat{d}\right)$; the latter is retrieved from the buffer $\mathbf{Z}_{c}$. Upon performing these calculations, CN stores the sequences $u(i), z(i)$ in the "temporary" buffers $\mathbf{U}_{c}^{\text {temp }}, \mathbf{Z}_{c}^{\text {temp}}$, respectively, and sends the content of the buffer $\mathbf{U}_{c}^{t e m p}$ to PN together with the value of $\hat{d}$. Once the packet is received, PN compares $\hat{d}$ with the actual RTT delay $d$. The fact $d>\hat{d}$ implies that the control sequence has arrived after the "deadline", and, therefore, the corresponding control sequence is considered outdated and to be discarded. If the control sequence arrived before $j_{p}^{*}+\hat{d}(j)$ (i.e., $d(j) \leq \hat{d}(j)$ ), it updates the input buffer $\mathbf{U}_{p}$ for $i \geq j+\hat{d}(j)$ but not for $i \in\{j+d(j), \ldots, j+\hat{d}(j)-1\}$ (a similar idea of using buffering for equalizing the variable delay to the worst-case delay was introduced in [96]).

The double set of buffers at $\mathrm{CN}$ is introduced to cope with possible packet losses. As explained above, CN initially stores the sequences $u(i), z(i)$ in the "temporary" buffers $\mathbf{U}_{c}^{\text {temp }}, \mathbf{Z}_{c}^{\text {temp }}$, respectively. If the control sequence successfully updated $\mathbf{U}_{p}$, the corresponding data are copied from $\mathbf{U}_{c}^{\text {temp }}, \mathbf{Z}_{c}^{\text {temp }}$ to $\mathbf{U}_{c}, \mathbf{Z}_{c}$. To indicate that a given control sequence has successfully updated $\mathbf{U}_{p}$, the protocol uses one-bit sequence numbers $n_{p}, n_{c}$. Once $\mathbf{U}_{p}$ is updated, $n_{p}$ is increased by one (modulo 2). The current value of $n_{p}$ is then sent with every measurements to CN. Upon arriving, $n_{p}$ is compared to a similar controller sequence number $n_{c}$. The fact that $n_{p} \neq n_{c}$ indicates that the previous control sequence arrives successfully to PN; in this case 
the corresponding data from $\mathbf{U}_{c}^{\text {temp }}, \mathbf{Z}_{c}^{\text {temp }}$ has to be written to permanent buffers $\mathbf{U}_{c}, \mathbf{Z}_{c}$, and $n_{c}$ is set equal to $n_{p}$. On the other hand, $n_{p}=n_{c}$ implies that the control sequence did not arrive, or was discarded by $\mathrm{PN}$; in this case, $\mathbf{U}_{c}, \mathbf{Z}_{c}$ are kept intact.

It is also possible that a control sequence has arrived to PN after a new measurement is performed and new data are already sent to CN. Such a control sequence may be misinterpreted and handled as the one based on the new measurement. Also, in this case the controller is already notified that the previous control sequence has not arrived, and the controller's buffers are overwritten with the new data. In order to recognize these "outdated" control sequences, the protocol uses parameters $\tau_{p}$ and $\tau_{c}$. More precisely, the parameter $\tau_{p}$ is then sent with the measurement data to $\mathrm{CN}$, and the parameter $\tau_{c}=\tau_{p}$ is returned to $\mathrm{PN}$, where $\tau_{c}$ is compared to the current value of $\tau_{p}$. In particular, $\tau_{c}<\tau_{p}$ implies that the control sequence is outdated, and has to be discarded.

Remark 12. Traditional real-time control algorithms require frequent exchange of small packets with strict real-time guarantees. The proposed protocol, on the other hand, relaxes the real-time requirements, and generally uses less frequent exchange of larger packets. This property makes it suitable for use in conjunction with traditional data networks, such as the Ethernet.

\subsubsection{Stability and Performance Recovery}

In order to give an analytical description of the model-based NCS with the above presented protocol, let us first introduce some additional notations. A measurement performed at $j \in \mathbb{J}_{0}$ is said to be successful if the corresponding control sequence arrives in time to the plant node and successfully updates the plant input buffer $\mathbf{U}_{p}$. The set of all successful measurement instants is denoted by $\mathbb{I}$, it forms a subset of 
the set $\mathbb{J}_{0}\left(\mathbb{J} \subset \mathbb{J}_{0}\right)$. By definition, $j \in \mathbb{J}$ if and only if $j \in \mathbb{J}_{0}$ and $d(j) \leq \hat{d}(j)$. Taking into account the protocol presented, one can formally consider a map $\Psi ; \mathbb{I} \rightarrow \mathbb{Z}^{+}$, $\Psi(j)=j+\hat{d}(j)$, which assigns to each successful measurement instant $j$ an instant when the corresponding control sequence is first applied to the plant's input. The range of $\Psi$ is denoted by $\Psi(\mathbb{J})$. Using this notation, the closed-loop model-based NCS can be described by the following set of equations

$$
\begin{aligned}
& x(i+1)=F^{+}(x(i), u(i), i), \\
& u(i)= \begin{cases}u^{0}(i) \in \mathcal{U}^{0} & \text { for } i<\Psi\left(j_{0}\right), \\
U_{h}(z(i), \bar{x}(i), i) & \text { for } i \in \Psi(\mathbb{J}), \\
U_{h}(z(i), \hat{x}(i), i) & \text { otherwise, }\end{cases} \\
& z(i+1)= \begin{cases}0 & \text { for } i<\Psi\left(j_{0}\right), \\
Z_{h}(z(i), \bar{x}(i), i) & \text { for } i \in \Psi(\mathbb{J}) \\
Z_{h}(z(i), \hat{x}(i), i) & \text { otherwise }\end{cases} \\
& \hat{x}(i+1)= \begin{cases}0 & \text { for } i<\Psi\left(j_{0}\right), \\
f_{h}^{+}(\bar{x}(i), u(i), i) & \text { for } i \in \Psi(\mathbb{J}), \\
f_{h}^{+}(\hat{x}(i), u(i), i) & \text { otherwise, }\end{cases}
\end{aligned}
$$

Here, $\bar{x}\left(\Psi\left(j_{k}\right)\right)$ is an estimate of $x\left(\Psi\left(j_{k}\right)\right)$ which is based on a measurement performed at $j_{k} \in \mathbb{J} ;$ it is defined as the value at $i=\Psi\left(j_{k}\right)$ of the solution of

$$
\bar{x}(i+1)=f_{h}^{+}(\bar{x}(i), u(i), i)
$$

with initial conditions $\bar{x}\left(j_{k}\right)=x\left(j_{k}\right)$. The following result is valid for NCS (5.10)(5.14).

Theorem 8. Consider NCS (5.10) - (5.14). Suppose Assumptions 9 and 10 hold. Given $\Delta_{x}>0, \delta>0, \delta \leq \Delta_{x}, \Delta_{u} \geq 0, \mathcal{T}_{\max } \in \mathbb{N}$, and $\alpha_{3}^{*} \in \mathcal{K}$ such that $\alpha_{3}(s)-\alpha_{3}^{*}(s)>0$ for $s>0$, suppose $x(0) \in \mathbb{R}^{n}$ and $\mathcal{U}^{0}:=\left\{u^{0}(0), u^{0}(1), \ldots\right\}$ are 
such that $\left|u^{0}(i)\right| \leq \Delta_{u}$ for all $i \in\left\{0,1, \ldots, \Psi\left(j_{0}\right)\right\}$, and the corresponding solution of (5.10) satisfies

$$
|x(i)| \leq \Delta_{x} \text { for all } i \in\left\{0,1, \ldots, \Psi\left(j_{0}\right)\right\} .
$$

Suppose also

$$
\Psi\left(j_{k}\right)-j_{k-1} \leq \mathcal{T}_{\max } \text { for all } j_{k} \in \mathbb{J} .
$$

Then there exists $h^{*}>0$ such that the system $(5.10)-(5.14)$ with initial conditions satisfying (5.15) is asymptotically stable with offset $\delta$ whenever $h \in\left(0, h^{*}\right]$. More precisely, the inequalities

$$
\begin{gathered}
\max \{|x(i)|,|z(i)|\} \leq \max \left\{\alpha_{1}^{-1} \circ \beta_{\alpha_{3}^{*}}\left(\alpha_{2}\left(\left|x\left(\Psi\left(j_{0}\right)\right)\right|\right), i-\Psi\left(j_{0}\right)\right), \delta / 2\right\}, \\
|x(i)-\hat{x}(i)| \leq \delta / 2
\end{gathered}
$$

hold for all $i \geq \Psi\left(j_{0}\right)$ and all $i>\Psi\left(j_{0}\right)$, respectively, where $\beta_{\alpha_{3}^{*}}\left(v_{0}, i\right)$ is a solution of the difference equation $v(i+1)=-\alpha_{3}^{*}(v(i))$ with initial condition $v(0)=v_{0}$.

Remark 13. Assumption 9 implies that the "ideal" decaying estimate for the non-networked system (5.1), (5.3) is $\alpha_{1}^{-1} \circ \beta_{\alpha_{3}}\left(\alpha_{2}(\cdot), i\right)$, where $\beta_{\alpha_{3}}(\cdot, i)$ is the solution of the equation $v(i+1)=-\alpha_{3}(v(i))$. Since the function $\beta_{\alpha_{3}^{*}}(\cdot, i)$ can be made arbitrarily close to $\beta_{\alpha_{3}}(\cdot, i)$ by choosing $\alpha_{3}^{*} \in \mathcal{K}$ sufficiently close to $\alpha_{3} \in \mathcal{K}$, Theorem 8 implies that the decaying estimate of the model-based NCS can be made arbitrarily close to the "ideal" one over arbitrarily large set of initial conditions, if $h>0$ is sufficiently small (i.e., the "performance recovery" [97] is achieved).

Remark 14. According to (5.16), the parameter $\mathcal{T}_{\max }$ is the upper bound to the sum of a transfer interval and the corresponding RTT delay. Theorem 8 states that $\mathcal{T}_{\text {max }}$ can be assigned arbitrarily by choosing $h>0$ sufficiently small. In particular, this implies, under the assumption that RTT delay is uniformly bounded, that the maximum allowable transfer interval (MATI [88]) can be assigned arbitrarily in the proposed model-based NCS. 
Remark 15. It was assumed above for simplicity that all the buffers involved have infinite capacity, and each packet successfully arrived to PN contains a control sequence with infinite number of elements. The important questions, however, are how much buffer capacity is actually required, and what is the minimum length of each control sequence that guarantee stability of the model-based NCS. From the protocol presented and the formulation of Theorem 8 , it follows that the required horizon of the control prediction (and, therefore, the required capacity of each of the buffers $\mathbf{U}_{p}, \mathbf{U}_{c}, \mathbf{U}_{c}^{\text {temp }}, \mathbf{Z}_{c}, \mathbf{Z}_{c}^{\text {temp }}$ ) is equal to $\mathcal{T}_{\max }$ elements, while the length of the corresponding control sequence must be greater than or equal to $\mathcal{T}_{\max }-\hat{d}$ elements to guarantee stability.

The proof of Theorem 8 utilizes the following simple lemma (for a similar result, see [53, Proposition 1]).

Lemma 1. Let $V: \mathbb{Z}^{+} \rightarrow R^{+}$be a sequence of nonnegative real numbers. Suppose there exist $\alpha \in \mathcal{K}, \alpha(s) \leq s$ for all $s \geq 0$, and $l_{1}, l_{2}, l_{3}$ satisfying $0 \leq$ $l_{1} \leq l_{2} \leq l_{3}$ such that $V(i+1)-V(i) \leq-\alpha(V(i))$ holds for all $V(i) \in\left[l_{1}, l_{3}\right]$, and $V(i+1)-V(i) \leq l_{2}-l_{1}$ for all $V(i) \in\left[0, l_{1}\right]$. Then $V(0) \leq l_{3}$ implies that $V(i) \leq \max \left\{\beta(V(0), i), l_{2}\right\}$ for all $i \in \mathbb{Z}^{+}$, where $\beta\left(V_{0}, i\right)$ is the solution of the difference equation $V(i+1)=V(i)-\alpha(V(i))$ with initial condition $V(0)=V_{0}$.

Proof of Theorem 8. Consider the system (5.10) - (5.14). First, according to Assumption 9 , for each $i>\Psi\left(j_{0}\right), i \notin \Psi(\mathbb{J})$, the inequality

$$
\Delta V_{h}(\hat{x}(i), z(i), i) \leq-\alpha_{3}\left(V_{h}(\hat{x}(i), z(i))\right)
$$

holds along the trajectories of $(\hat{x}, z)$-subsystem. In particular, this implies that the 
inequalities

$$
\begin{gathered}
\Delta V_{h}(x, z, i)-\left|V_{h}(\hat{x}(i), z(i))-V_{h}(x(i), z(i))\right| \\
-\left|V_{h}(\hat{x}(i+1), z(i+1))-V_{h}(x(i+1), z(i+1))\right| \\
\leq \Delta V_{h}(\hat{x}(i), z(i), i) \leq-\alpha_{3}\left(V_{h}(\hat{x}(i), z(i))-V_{h}(x(i), z(i))+V_{h}(x(i), z(i))\right) \\
\leq-\alpha_{3} \circ[\mathbb{I}-\epsilon]\left(V_{h}(x(i), z(i))\right)+\alpha_{3} \circ\left[\epsilon^{-1}+\mathbb{I}\right]\left(\left|V_{h}(\hat{x}(i), z(i))-V_{h}(x(i), z(i))\right|\right)
\end{gathered}
$$

hold for each $i>\Psi\left(j_{0}\right), i \notin \Psi(\mathbb{J})$, where $\mathbb{I}(s)=s$ for all $s \geq 0$, and $\epsilon(\cdot) \in \mathcal{K}_{\infty}$ is such that $[\mathbb{I}-\epsilon] \in \mathcal{K}_{\infty}$. On the other hand, Assumption 9 implies that the inequality

$$
V_{h}(\hat{x}(i+1), z(i+1))-V_{h}(\bar{x}(i), z(i)) \leq-\alpha_{3}\left(V_{h}(\bar{x}(i), z(i))\right)
$$

holds for all $i \in \Psi(\mathbb{J})$. Using the same line of reasoning as above, we see that

$$
\begin{gathered}
\Delta V_{h}(x, z, i)-\left|V_{h}(\bar{x}(i), z(i))-V_{h}(x(i), z(i))\right| \\
-\left|V_{h}(\hat{x}(i+1), z(i+1))-V_{h}(x(i+1), z(i+1))\right| \\
\leq-\alpha_{3} \circ[\mathbb{I}-\epsilon]\left(V_{h}(x(i), z(i))\right)+\alpha_{3} \circ\left[\epsilon^{-1}+\mathbb{I}\right]\left(\left|V_{h}(\bar{x}(i), z(i))-V_{h}(x(i), z(i))\right|\right)
\end{gathered}
$$

holds for each $i \in \Psi(\mathbb{J})$. The following claims are valid.

Claim 1. Given $\Delta_{x}^{*} \geq 0, \Delta_{z}^{*} \geq 0, \Delta_{u}^{*} \geq 0$, there exists $\varrho(i, h)$ which is a $\mathcal{K}$-class function in $h>0$ and strictly increasing in $i \in \mathbb{N}$ such that the following holds. Suppose

$$
|x(i)| \leq \Delta_{x}, \quad|u(i)| \leq \Delta_{u}, \quad|z(i)| \leq \Delta_{z}
$$

hold for all $i \leq \Psi\left(j_{k}\right)$, where $j_{k} \in \mathbb{J}$. Then

$$
\begin{aligned}
\left|x\left(\Psi\left(j_{k}\right)\right)-\bar{x}\left(\Psi\left(j_{k}\right)\right)\right| & \leq \varrho\left(\Psi\left(j_{k}\right)-j_{k}, h\right) \\
|x(i)-\hat{x}(i)| & \leq \varrho\left(\Psi\left(j_{k+1}\right)-j_{k}, h\right)
\end{aligned}
$$

where (5.25) holds for all $i \in\left\{\Psi\left(j_{k}\right)+1, \ldots, \Psi\left(j_{k+1}\right)\right\}$.

Proof. First, Assumption 10 together with (5.23) imply that

$$
\left|x\left(\Psi\left(j_{k}\right)\right)-\bar{x}\left(\Psi\left(j_{k}\right)\right)\right| \leq \rho_{\Delta_{x}^{*}, \Delta_{u}^{*}}\left(\Psi\left(j_{k}\right)-j_{k}, h\right) .
$$


Next, (5.19), (5.21) imply that

$V_{h}(\hat{x}(i), z(i)) \leq V_{h}\left(\bar{x}\left(\Psi\left(j_{k}\right)\right), z\left(\Psi\left(j_{k}\right)\right)\right)$ for all $i \in\left\{\Psi\left(j_{k}\right)+1, \ldots, \Psi\left(j_{k+1}\right)\right\}$. Since $\left|\bar{x}\left(\Psi\left(j_{k}\right)\right)\right| \leq \Delta_{x}^{*}+\rho_{\Delta_{x}^{*}, \Delta_{u}^{*}}\left(\Psi\left(j_{k}\right)-j_{k}, h^{*}\right)$, and $\left|z\left(\Psi\left(j_{k}\right)\right)\right| \leq \Delta_{z}^{*}$, Assumption 9 (more precisely, inequality (5.4)) implies that

$$
\max \{|\hat{x}(i)|,|z(i)|\} \leq \alpha_{1}^{-1} \circ \alpha_{2}\left(\max \left\{\Delta_{x}^{*}+\rho_{\Delta_{x}^{*}, \Delta_{u}^{*}}\left(\Psi\left(j_{k}\right)-j_{k}, h^{*}\right), \Delta_{z}^{*}\right\}\right)
$$

for all $i \in\left\{\Psi\left(j_{k}\right)+1, \ldots, \Psi\left(j_{k+1}\right)\right\}$. Further, according to Assumption $9, U_{h}(z, \hat{x}, i)$ is bounded on an arbitrary bounded set of $z, \hat{x}$ uniformly in $h \in\left(0, h^{*}\right]$ and $i \in \mathbb{Z}^{+}$, which implies that $u(i)=U_{h}(z(i), \hat{x}(i), i)$ is uniformly bounded on $\left\{\Psi\left(j_{k}\right)+1, \ldots, \Psi\left(j_{k+1}\right)\right\}$ with some upper bound $\Delta_{u}^{\sharp}$. Choose $\Delta_{u}^{0}:=\max \left\{\Delta_{u}^{*}, \Delta_{u}^{\sharp}\right\}$. Due to Assumption 10, (5.24) and (5.25) hold with $\varrho(\cdot, \cdot):=\rho_{\Delta_{x}^{*}, \Delta_{u}^{0}}(\cdot, \cdot)$.

Claim 2. Given $\Delta_{x}^{*} \geq 0, \Delta_{z}^{*} \geq 0, \Delta_{u}^{*} \geq 0$, there exists $\varrho_{1}(i, h)$ which is a $\mathcal{K}$-class function in $h>0$ and strictly increasing in $i \in \mathbb{N}$ such that the following holds. Suppose (5.23) is valid for some $j_{k} \in \mathbb{J}$, then

$$
\begin{aligned}
& \Delta V_{h}(x, z, i) \leq-\alpha_{3} \circ[\mathbb{I}-\epsilon]\left(V_{h}(x(i), z(i))\right) \\
& +\left[\alpha_{3} \circ\left[\epsilon^{-1}+\mathbb{I}\right]+2 \mathbb{I}\right] \circ \varrho_{1}\left(\Psi\left(j_{k+1}\right)-j_{k}, h\right),
\end{aligned}
$$

holds for all $i \in\left\{\Psi\left(j_{k}\right), \ldots, \Psi\left(j_{k+1}-1\right)\right\}$, where $\epsilon(\cdot) \in \mathcal{K}_{\infty}$ is such that $[\mathbb{I}-\epsilon] \in$ $\mathcal{K}_{\infty}$

Proof. It was shown above in the proof of Claim 1 that all the signals $x(i)$, $\hat{x}(i), z(i)$ are uniformly bounded for all $i \in\left\{\Psi\left(j_{k}\right), \ldots, \Psi\left(j_{k+1}-1\right)\right\}$. Taking into account the uniform continuity of $V_{h}$ as well as inequalities (5.20), (5.22), the statement of Claim 2 follows.

Claim 3. Under the assumptions of Theorem 8 , there exists $h^{*}>0$ such that for any $h \in\left(0, h^{*}\right]$ the inequalities

$$
\Delta V_{h}(x, z, i) \leq-\alpha_{3} \circ[\mathbb{I}-\epsilon]\left(V_{h}(x(i), z(i))\right)+\left[\alpha_{3} \circ\left[\epsilon^{-1}+\mathbb{I}\right]+2 \mathbb{I}\right] \circ \varrho_{1}\left(\mathcal{T}_{\max }, h\right),
$$




$$
|x(i+1)-\hat{x}(i+1)| \leq \varrho\left(\mathcal{T}_{\max }, h\right)
$$

hold for all $i \geq \Psi\left(j_{0}\right)$.

Proof. Given $\Delta_{x}>0, \Delta_{u} \geq 0$, put $\Delta_{x}^{*}:=\Delta_{z}^{*}=\alpha_{1}^{-1} \circ \alpha_{2}\left(\Delta_{x}\right)$. Furthermore, choose $\Delta_{u}^{0} \geq 0$ such that $\left|U_{h}(z, \hat{x}, i)\right| \leq \Delta_{u}^{0}$ for all $i \in \mathbb{Z}^{+}$as long as $|z| \leq \Delta_{z}^{*}$ and $\hat{x} \leq$ $2 \Delta_{x}^{*}$, and put $\Delta_{u}^{*}:=\max \left\{\Delta_{u}, \Delta_{u}^{0}\right\}$. By Claim 1 , let $\varrho(\cdot, \cdot)$ be generated by $\Delta_{x}^{*}, \Delta_{z}^{*}$, $\Delta_{u^{*}}^{*}$. Also, by Claim 2 , let $\varrho_{1}(\cdot, \cdot)$ be generated by $\Delta_{x}^{*}, \Delta_{z}^{*}, \Delta_{u}^{*}$. Finally, choose $h^{*}>0$ such that $\varrho\left(\mathcal{T}_{\max }, h^{*}\right) \leq \Delta_{x}^{*}$ and $1 / 4 \cdot \alpha_{3} \circ[\mathbb{I}-\epsilon] \circ \alpha_{2}\left(\Delta_{x}\right) \geq\left[\alpha_{3} \circ\left[\epsilon^{-1}+\mathbb{I}\right]+2 \mathbb{I}\right] \circ$ $\varrho_{1}\left(\mathcal{T}_{\max }, h^{*}\right)$. Suppose $|x(i)| \leq \Delta_{x},\left|u^{0}(i)\right| \leq \Delta_{u}$ hold for all $i \leq \Psi\left(j_{0}\right)$. Claim 2 then implies that (5.27) holds at least for $i \in\left\{\Psi\left(j_{0}\right), \ldots, \Psi\left(j_{1}-1\right)\right\}$. Further, since $|x(i)| \leq \Delta_{x}$ and $|z(i)|=0$ for all $i \leq \Psi\left(j_{0}\right)$, inequality (5.27) then implies that $V_{h}(i) \leq \alpha_{2}\left(\Delta_{x}\right)$ for all $i \leq \Psi\left(j_{1}\right)$, or $|x(i)| \leq \Delta_{x}^{*}$, and $|z(i)| \leq \Delta_{z}^{*}$ for all $i \leq \Psi\left(j_{1}\right)$. Also, $\varrho\left(\mathcal{T}_{\max }, h^{*}\right) \leq \Delta_{x}^{*}$ together with (5.25) imply that $\hat{x}(i) \leq 2 \Delta_{x}^{*}$ for all $i \leq \Psi\left(j_{1}\right)$. Finally, by the choice of $\Delta_{u}^{*}$, we have $\left|U_{h}(z(i), \hat{x}(i), i)\right| \leq \Delta_{u}^{*}$ for all $i \leq \Psi\left(j_{1}\right)$. Applying Claim 2, we see that (5.27) is also valid for all $i \in\left\{\Psi\left(j_{1}\right), \ldots, \Psi\left(j_{2}-1\right)\right\}$, etc. The statement of Claim 3 now follows by induction arguments.

Now, the proof of Theorem 8 can be finalized as follows. Given $\Delta_{x} \geq \delta>0$, choose $\epsilon \in \mathcal{K}_{\infty}$ and $h^{*}>0$ such that $\left[\alpha_{3} \circ\left[\epsilon^{-1}+\mathbb{I}\right]+2 \mathbb{I}\right] \circ \varrho_{1}\left(\mathcal{T}_{\max }, h^{*}\right) \leq \frac{1}{4} \alpha_{1}(\delta)$, $\varrho\left(\mathcal{T}_{\max }, h^{*}\right) \leq \delta / 2$, and the inequality $\alpha_{3} \circ[\mathbb{I}-\epsilon](s) \geq \alpha_{3}^{*}(s)+\left[\alpha_{3} \circ\left[\epsilon^{-1}+\mathbb{I}\right]+2 \mathbb{I}\right] \circ$ $\varrho_{1}\left(\mathcal{T}_{\max }, h^{*}\right)$ holds for all $s \in\left[\frac{1}{4} \alpha_{1}(\delta), \alpha_{2}\left(\Delta_{x}\right)\right]$. Note that such a choice is always possible. Inequality (5.27) now implies that $\Delta V_{h}(x, z, i) \leq-\alpha_{3}^{*}\left(V_{h}(x(i), z(i))\right)$ holds for all $V_{h}(x(i), z(i)) \in\left[\frac{1}{4} \alpha_{1}(\delta), \alpha_{2}\left(\Delta_{x}\right)\right]$, and $\Delta V_{h}(x, z, i) \leq 1 / 4 \cdot \alpha_{1}(\delta)$ whenever $V_{h}(x(i), z(i)) \in\left[0, \frac{1}{4} \alpha_{1}(\delta)\right]$. Applying Lemma 1, we see that (5.17) holds for all $i \geq \Psi\left(j_{0}\right)$. Also, (5.28) implies (5.18). The proof of Theorem 8 is complete. 


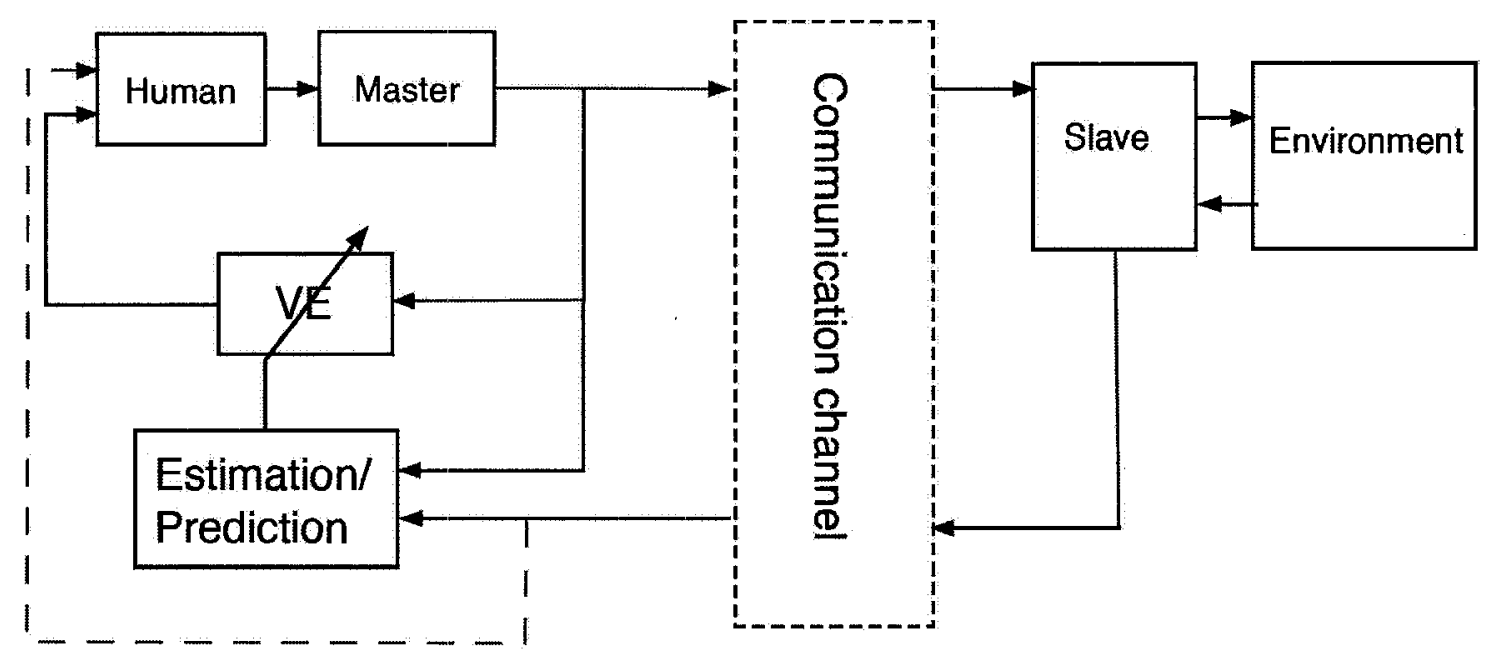

Figure 5.1: Structure of VE-enhanced teleoperator system

\subsection{Virtual Reality Enhanced Bilateral Teleoper- ation with Communication Constraints}

In this section, we apply the ideas presented above to the design of virtual environment based teleoperator systems. A general structure of the virtual environment based force-reflecting teleoperator system is shown in Figure 5.1. The need for virtual environment on the master side appears usually when communications between the parts of telerobot are subject to communication constraints significant enough to make it impossible to successfully control the telerobot using direct force feedback (shown by dashed line in Figure 5.1). One particularly important case is where significant communication delays and/or data losses arise which destroy the sense of causality for the human operator, and may also lead to overall instability. In this case, a virtual slave+environment (VE) interconnection is created on the master side which duplicates essential features of the remote slave-environment interconnection. This virtual slave+environment block may be used to provide the human with some 
force feedback (as well as some other type of feedback); since this feedback is not subject to communication delay and other communication constraints, it may restore the sense of causality and, when properly designed, guarantee stability of the overall system. This type of feedback, however, is prone to modeling errors. In order to make this virtual environment provide the human operator with a response similar to the one from the real remote environment, the state of virtual environment should be updated frequently based on (possibly delayed) measurement of the remote environment response and the corresponding state estimation/prediction algorithms.

The outline of our approach is as follows. We assume that a partially known approximate discrete-time model of the remote slave/environment interconnection is available for the designer. This model is implemented in the virtual environment on the master side together with certain observation/identification/prediction mechanism that allows to approximately recover the "current" state/parameters of the remote environment based on delayed and possibly distorted measurement data. We develop a control/communication protocol that allows to guarantee the stability/tracking properties of the teleoperator system in presence of network induced communication constraints. Below, the corresponding theoretical framework is presented.

\subsubsection{Approximate Discrete-Time Model Based Design}

Consider a teleoperation system where both the master and the "slave+environment" subsystems are described as nonlinear systems of the form

$$
\begin{aligned}
& \dot{x}_{m}=F_{m}\left(x_{m}, u_{m}, u_{h}\right) \\
& y_{m}=G_{m}\left(x_{m}, u_{m}, u_{h}\right)
\end{aligned}
$$


and

$$
\begin{aligned}
& \dot{x}_{s}=F_{s}\left(x_{s}, u_{s}, \theta_{s}\right) \\
& y_{s}=G_{s}\left(x_{s}, u_{s}, \theta_{s}\right),
\end{aligned}
$$

respectively. Here $x_{m}, x_{s}$ are states, $u_{m}$ and $u_{s}$ are local controller inputs, and $y_{m}, y_{s}$ are the outputs of the master robot and the "slave+environment" subsystem, respectively; $u_{H}$ is the input applied by the human operator to the master robot, and $\theta$ is a (constant) vector of parameters of the remote environment, which is assumed to be unknown but belongs to some (compact) subset $\Theta_{s}$. We address the situation, where the master and the slave subsystems communicate over a digital network, therefore, it is convenient to consider the teleoperator problem in the discrete-time setting. Given a sampling period $T>0$, the subsystems (5.29) and (5.30) can be described by their exact discrete-time models, as follows,

$$
\begin{aligned}
x_{m}(i+1) & =F_{m}^{+}\left(x_{m}(i), u_{m}(i), u_{H}(i)\right) \\
y_{m}(i) & =G_{m}^{+}\left(x_{m}(i), u_{m}(i), u_{H}(i)\right),
\end{aligned}
$$

and

$$
\begin{aligned}
x_{s}(i+1) & =F_{s}^{+}\left(x_{s}(i), u_{s}(i), \theta_{s}(i)\right) \\
\theta_{s}(i+1) & =\theta_{s}(i) \\
y_{s}(i) & =G_{s}\left(x_{s}(i), u_{s}(i)\right),
\end{aligned}
$$

respectively. In virtual environment (VE) based teleoperator systems, a model of the remote subsystem is included into the local virtual environment, so the human operator receives the force feedback (and possibly other forms of feedback) without being subject to communication constraints. For nonlinear systems of the form (5.29), (5.30), the exact-discrete time model is usually unknown, since the modelling process involves exact integration of the underlying nonlinear differential equations, which is generally not possible. Instead, a set of approximate models parameterized by a modelling parameter $h>0$ is usually available (a common example of such 
a model is the discrete-time Euler approximation of the corresponding continuoustime dynamics). Therefore, we assume that a set of approximate models of the remote subsystem (5.30) of the form

$$
\begin{aligned}
x_{s, h}(i+1) & =f_{s, h}^{+}\left(x_{s, h}(i), u_{s, h}(i), \theta_{s, h}(i)\right) \\
\theta_{s, h}(i+1) & =\theta_{s, h}(i), \\
y_{s, h}(i) & =g_{s, h}^{+}\left(x_{s, h}(i), u_{s, h}(i), \theta_{s, h}(i)\right)
\end{aligned}
$$

is implemented on the master side, where $h>0$ is the modelling parameter. The master subsystem forms an interconnection with the virtual environment (5.33), as follows

$$
u_{s, h}=y_{m}, \quad u_{m}=y_{s, h} .
$$

The human operator is, therefore, assumed to apply forces to the master subsystem that depend on the state of the master manipulator and the state of VR (5.33), as well as the parameters of the VR, according to the formula

$$
u_{H}=U_{H}\left(x_{m}, x_{s, h}, \theta_{s, h}\right) .
$$

Let the following set of assumptions be valid for the system (5.31)-(5.35). First, it is assumed that human operator (5.35) is able to stabilize the closed-loop "master+VR" subsystem (5.29), (5.33), (5.34) uniformly for all $\theta_{s, h} \in \Theta$. More precisely, this assumption can be formulated in terms of Lyapunov-like conditions as follows.

Assumption 11. The human operator (5.35) stabilizes the closed-loop "master+VR" subsystem (5.29), (5.33), (5.34) uniformly for all $\theta_{s, h} \in \Theta$. More precisely, there exists a set of Lyapunov functions $V_{h}: \mathbb{R}^{n 1} \times \mathbb{R}^{n 2} \rightarrow \mathbb{R}_{+}, h \in\left(0, h^{*}\right]$, which are locally Lipschitz uniformly in $h \in\left(0, h^{*}\right]$, such that

$$
\alpha_{1}\left(\max \left\{\left|x_{m}\right|,\left|x_{s, h}\right|\right\}\right) \leq V_{h}\left(x_{m}, x_{s, h}\right) \leq \alpha_{2}\left(\max \left\{\left|x_{m}\right|,\left|x_{s, h}\right|\right\}\right)
$$

and the inequality

$$
\Delta V_{h}(\cdot) \leq-\alpha_{3}\left(V_{h}\left(x_{m}(i), x_{s, h}(i)\right)\right)
$$


holds along the trajectories of the system (5.35), (5.29), (5.33), (5.34), where $\Delta V_{h}(i):=$ $V_{h}\left(x_{m}(i+1), x_{s, h}(i+1)\right)-V_{h}\left(x_{m}(i), x_{s, h}(i)\right)$.

Furthermore, it is assumed that the approximate discrete-time model used in the virtual environment provides consistent approximation of the remote slave+environment dynamics, in the following sense.

Assumption 12. (Consistency of approximation) Given $\Delta_{x}, \Delta_{u} \geq 0$, there exist $\rho_{f}(\cdot), \rho_{g}(\cdot) \in \mathcal{K}, \lambda_{x}, \lambda_{\theta} \in \mathcal{K}$, and $h^{*}>0$, such that the following two properties hold:

i) One-Step Time-Uniform Consistency of the Approximation Scheme: for any $x_{s} \in \bar{B}\left(\Delta_{x}\right)$, any $u_{s} \in \bar{B}\left(\Delta_{u}\right)$, any $\theta_{s} \in \Theta_{s}$, and each $h \in\left(0, h^{*}\right]$,

$$
\begin{array}{r}
\left|F_{s}^{+}\left(x_{s}, u_{s}, \theta_{s}\right)-f_{s, h}^{+}\left(x_{s}, u_{s}, \theta_{s}\right)\right| \leq \rho_{f}(h) ; \\
\left|G_{s}\left(x_{s}, u_{s}, \theta_{s}\right)-g_{s, h}^{+}\left(x_{s}, u_{s}, \theta_{s}\right)\right| \leq \rho_{g}(h) ;
\end{array}
$$

ii) Uniform Continuity in $x, \theta$ of Approximate Models: for any $x_{1}, x_{2} \in \bar{B}\left(\Delta_{x}\right)$, $\theta_{1}, \theta_{2} \in \Theta_{s}, u \in \bar{B}\left(\Delta_{u}\right)$, and $h \in\left(0, h^{*}\right]$,

$$
\left|f_{s, h}^{+}\left(x_{1}, u, \theta_{1}\right)-f_{s, h}^{+}\left(x_{2}, u, \theta_{2}\right)\right| \leq \lambda_{x}\left(\left|x_{1}-x_{2}\right|\right)+\lambda_{\theta}\left(\left|\theta_{1}-\theta_{2}\right|\right) \cdot \bullet
$$

Also, the approximate dynamics (5.33) are assumed to be observable/identifiable in the following sense.

Assumption 13. (Uniform observability of the slave's approximate models) There exist a set of observers of the form

$$
\begin{aligned}
\hat{x}(i+1) & =f_{s, h}^{+}\left(\hat{x}(i), u_{s, h}(i), \hat{\theta}(i)\right)+\gamma_{\hat{x}}\left(\hat{x}(i), \hat{\theta}(i), u_{s, h}(i), y_{s, h}(i)\right) \\
\hat{\theta}(i+1) & =\hat{\theta}(i)+\gamma_{\hat{\theta}}\left(\hat{x}(i), \hat{\theta}(i), u_{s, h}(i), y_{s, h}(i)\right),
\end{aligned}
$$

where $\gamma_{\hat{x}}(\cdot), \gamma_{\hat{\theta}}(\cdot)$ are continuous functions of their arguments, and a family of Lyapunov functions $V_{h}^{o}(x, \hat{x}, \theta, \hat{\theta}), h \in\left(0, h^{*}\right]$, such that $V_{h}^{o}(x, \hat{x}, \theta, \hat{\theta})$ are continuous in $x, \hat{x}, \theta, \hat{\theta}$ uniformly for $h \in\left(0, h^{*}\right]$, and

$$
\alpha_{1}^{o}(\max \{|\hat{x}-x|,|\hat{\theta}-\theta|\}) \leq V_{h}^{o}(x, \hat{x}, \theta, \hat{\theta}) \leq \alpha_{2}(\max \{|\hat{x}-x|,|\hat{\theta}-\theta|\})
$$


holds for some $\alpha_{1}^{o}, \alpha_{2}^{o} \in \mathcal{K}_{\infty}$, such that the following holds. Given $a^{*} \in(0,1)$, $\delta_{*}^{o}, \Delta_{*}^{o} \in(0,+\infty), \delta_{*}^{o}<\Delta_{*}^{o}$, there exist "output injection" functions $\gamma_{\hat{x}}(\cdot), \gamma_{\hat{\theta}}(\cdot)$ in (5.41) such that

$$
\Delta V_{h}^{o}(\cdot) \leq-\alpha_{3}^{o}\left(V_{h}^{o}\left(x_{s, h}(i), \hat{x}(i), \theta_{s, h}, \hat{\theta}(i)\right)\right)
$$

holds along the trajectories of the system (5.33), (5.41) with some $\alpha_{3}^{o} \in \mathcal{K}_{\infty}$ satisfying $\alpha_{3}^{o}(s)<s$ for all $s>0$ and $a^{*} s \leq \alpha_{3}^{o}(s)$ for $s \in\left[\delta_{*}^{o}, \Delta_{*}^{o}\right]$.

The last assumption is related to the communication process between the master subsystem and the remote slave+environment subsystem. We address a situation where the communication is subject to communication delay in both directions, and moreover, some of the packets may be lost or corrupted. However, we do assume that, within some fixed period of time, the communicated signal may be recovered on the receiver side with arbitrarily small error. More formally, the following assumption is valid.

Assumption 14. (Communication) Given arbitrary $\delta_{m}>0, \delta_{s}>0$, there exist $T_{f}, T_{b} \in \mathbb{Z}_{+}$such that the following relations are valid between the signals in the master (5.31) and the slave+environment (5.32) subsystems

$$
\begin{aligned}
\left|u_{s}(i)-y_{m}\left(i-T_{f}\right)\right| & \leq \delta_{s}, \\
\left|\hat{y}_{s}(i)-y_{s}\left(i-T_{b}\right)\right| & \leq \delta_{m},
\end{aligned}
$$

where $\hat{y}_{s}$ is the signal used below to update the state of the virtual environment.

Now, suppose the master node is equipped with two buffers, $\mathcal{Y}_{m}$ and $\mathcal{Y}_{s}$, where the sequences of signals $y_{m}(\cdot)$ and $\hat{y}_{s}(\cdot)$ are stored, respectively. More precisely, the capacity of these buffers should allow to store $T$ last signals, where $T:=T_{f}+T_{b}$ is the round-trip time delay.

The control/communication protocol for the master node can be described as follows. 
Protocol for the Master Node:

Initialization: $\hat{x}_{s, h}(0)=0, \hat{\theta}_{s, h}(0)=\theta^{*} \in \Theta_{s}, T_{o} \in \mathbb{N}$;

for $i \in \mathbb{Z}^{+}$

measure $y_{m}(i)$;

update $\mathcal{Y}_{m}$ with $\left\{y_{m}(i), i\right\}$

send $\left\{y_{m}(i), i\right\}$ to the SN

if $i \geq T$

retrieve $y_{m}(i-T)$ from $\mathcal{Y}_{m}$ and $\hat{y}_{s}(i)$ from $\mathcal{Y}_{s}$;

obtain $\hat{x}_{s, h}(i-T+1), \hat{\theta}_{s, h}(i-T+1)$ as a solution of the iterative observation/identification equation

$$
\begin{aligned}
\hat{x}_{s, h}(i-T+1)= & f_{s, h}^{+}\left(\hat{x}_{s, h}(i-T), y_{m}(i-T), \hat{\theta}(i-T)\right) \\
& +\gamma_{\hat{x}}\left(\hat{x}_{s, h}(i-T), \hat{\theta}(i-T), y_{m}(i-T), \hat{y}_{s}(i)\right), \\
\hat{\theta}_{s, h}(i-T+1)= & \hat{\theta}(i-T)+\gamma_{\hat{\theta}}\left(\hat{x}_{s, h}(i-T), \hat{\theta}(i-T), y_{m}(i-T), \hat{y}_{s}(i)\right) .
\end{aligned}
$$

if $i \geq T+T_{0}$

retrieve $y_{m}(j), j \in\{i-T+1, \ldots i\}$ from $\mathcal{Y}_{m}$

calculate $\tilde{x}_{s, h}(i)$ as a solution of the equation

$$
\tilde{x}_{s, h}(j+1)=f_{s, h}^{+}\left(\tilde{x}_{s, h}(j), y_{m}(j), \hat{\theta}(i-T)\right)
$$

with "initial" conditions $\tilde{x}_{s, h}(i-T) \leftarrow \hat{x}_{s, h}(i-T)$.

if either $\left|\tilde{x}_{s, h}(i)-x_{s, h}(i)\right|>\delta_{x}$ or $|\theta-\hat{\theta}(i-T)|>\delta_{\theta}$ :

overwrite $\theta \leftarrow \hat{\theta}(i-T), x_{s, h}(i) \leftarrow \tilde{x}_{s, h}(i)$

end if 
end if

end if

end for

The communication protocol for the slave-environment node is much simpler; it is presented below.

\section{Protocol for the Slave Node (SN):}

for $i \in \mathbb{Z}^{+}$

control action $u_{s}(i)$ is applied to the input of the slave;

measure $y_{s}(i)$;

send $\left\{y_{s}(i), i\right\}$ to the $\mathrm{MN}$;

end for

The above described VR-based teleoperator system can be made stable by choosing the modelling parameter $h>0$ sufficiently small. More precisely, the following statement is valid.

Theorem 9. Consider the VR-based teleoperator system (5.31)-(5.35) with control/communication protocols described above. Suppose Assumptions 11-14 hold. Then, for any given $T_{f}, T_{b} \in \mathbb{Z}_{+}$, the system can be made stable by an appropriate choice of design parameters. More precisely, given $\delta_{x}, \Delta_{x}>0, \delta_{x} \leq \Delta_{x}$, there exist $h^{*}>0, \delta_{m}, \delta_{s}, \delta_{x}, \delta_{\theta}>0$, and "output injection" functions $\gamma_{\hat{x}}(\cdot), \gamma_{\hat{\theta}}(\cdot)$ in (5.41) such that the assumption

$$
\max \left\{\left|x_{m}(0)\right|,\left|x_{s}\left(T_{f}\right)\right|\right\} \leq \Delta_{x}
$$

implies that

$$
\begin{gathered}
\max \left\{\left|x_{m}(i)\right|,\left|x_{s}\left(i+T_{f}\right)\right|,\left|x_{s, h}(i)\right|\right\} \\
\leq \max \left\{\beta\left(\max \left\{\left|x_{m}(0)\right|,\left|x_{s}\left(T_{f}\right)\right|\right\}, i\right), \delta_{x}\right\}
\end{gathered}
$$


holds along the trajectories of (5.31)-(5.35) for all $i \in \mathbb{N}$ whenever $h \in\left(0, h^{*}\right]$.

\subsubsection{Proof of Theorem 9}

Lemma 2. There exists $\beta_{1} \in \mathcal{N}_{\infty}$ such that for any $\Delta_{x} \geq 0$, assumption $\max \left\{\left|x_{m}(0)\right|,\left|x_{s}\left(0+T_{f}\right)\right|,\left|x_{s, h}(0)\right|\right\} \leq \Delta_{x}$ implies

$$
\max \left\{\left|x_{m}(i)\right|,\left|x_{s}\left(i+T_{f}\right)\right|,\left|x_{s, h}(i)\right|\right\} \leq \beta_{1}\left(\Delta_{x}\right) \text { for all } i \in\left\{0, \ldots, T+T_{o}\right\}
$$

Proof. First, consider the time interval $\left\{0, \ldots, T+T_{o}\right\}$. On this interval, the state of VR is not updated based on the remote measurement; thus, the solutions of the closed-loop "Master+VR" subsystems satisfy Assumption 11. In particular, this implies that $x_{m}(i), x_{s, h}(i)$ are uniformly bounded for $i \in\left\{0, \ldots, T+T_{o}\right\}$ with upper bound equal to $\alpha^{-1} \circ \alpha_{2}\left(\Delta_{x}\right)$, and by continuity considerations this also implies the uniform boundedness of all signals of the master $+V R$ subsystem, in particular, the uniform boundedness of $y_{m}(i)$. Taking into account Assumption 14, this also implies the uniform boundedness of $u_{s}\left(i+T_{f}\right)$. Finally, continuity considerations as well as forward completeness of the slave subsystem imply that $x_{s}\left(i+T_{f}\right)$ is uniformly bounded on $\left\{0, \ldots, T+T_{o}\right\}$ by $\beta_{1}\left(\Delta_{x}\right)$, where $\beta_{1}$ is some $\mathcal{N}_{\infty}$-class function.

Lemma 3. Given $\Delta_{x}^{*}>0, \epsilon_{s}>0, t_{1} \geq T+T_{o}$, there exist "output injection" functions $\gamma_{\hat{x}}, \gamma_{\hat{\theta}}$ in (5.43) such that the following holds. Suppose

$$
\max \left\{\left|x_{m}(i)\right|,\left|x_{s}\left(i+T_{f}\right)\right|,\left|x_{s, h}(i)\right|\right\} \leq \Delta_{x}^{*}, \quad \text { for all } i \in\left\{0, \ldots, t_{1}\right\}
$$

Then

$$
\begin{gathered}
\max \left\{\left|x_{s, h}\left(t_{1}\right)-x_{s}\left(t_{1}+T_{f}\right)\right|,\left|x_{s, h}\left(t_{1}+1\right)-x_{s}\left(t_{1}+T_{f}+1\right)\right|\right\} \\
\leq \rho_{h}^{*}(h)+\gamma_{\delta s}\left(\delta_{s}\right)+\gamma_{\delta m}\left(\delta_{m}\right)+\delta_{x}+\epsilon_{s}
\end{gathered}
$$

for some $\rho_{h}^{*}(\cdot), \gamma_{\delta s}(\cdot), \gamma_{\delta m}(\cdot) \in \mathcal{K}$ 
Proof. First, let us assume that

$$
\left|\tilde{x}_{s, h}\left(t_{1}-T\right)-x_{s}\left(t_{1}-T+T_{f}\right)\right| \leq \epsilon_{x}, \quad\left|\hat{\theta}\left(t_{1}-T\right)-\theta_{s}\right| \leq \epsilon_{\theta},
$$

holds for sufficiently small $\epsilon_{x}, \epsilon_{\theta}>0$. Using Assumptions 12, 14, one can easily derive that

$$
\begin{gathered}
\max \left\{\left|\tilde{x}_{s, h}\left(t_{1}\right)-x_{s}\left(t_{1}+T_{f}\right)\right|,\left|\tilde{x}_{s, h}\left(t_{1}+1\right)-x_{s}\left(t_{1}+T_{f}+1\right)\right|\right\} \\
\leq \rho_{h}^{*}(h)+\gamma_{\delta s}\left(\delta_{s}\right)+\gamma_{\delta m}\left(\delta_{m}\right)+\gamma_{x}\left(\epsilon_{x}\right)+\gamma_{\theta}\left(\epsilon_{\theta}\right),
\end{gathered}
$$

holds for some $\rho_{h}^{*}(\cdot), \gamma_{\delta s}(\cdot), \gamma_{\delta m}(\cdot), \gamma_{x}(\cdot), \gamma_{\theta}(\cdot) \in \mathcal{K}$, where $\tilde{x}_{s, h}(i)$ is the corresponding solution of $(5.44)$. Note that, according to the master node protocol, $x_{s, h}(i)$ is rewritten with $\tilde{x}_{s, h}(i)$ if $\left|\tilde{x}_{s, h}(i)-x_{s, h}(i)\right|>\delta_{x}$; therefore

$$
\begin{gathered}
\max \left\{\left|x_{s, h}\left(t_{1}\right)-x_{s}\left(t_{1}+T_{f}\right)\right|,\left|x_{s, h}\left(t_{1}+1\right)-x_{s}\left(t_{1}+T_{f}+1\right)\right|\right\} \\
\leq \rho_{h}^{*}(h)+\gamma_{\delta s}\left(\delta_{s}\right)+\gamma_{\delta m}\left(\delta_{m}\right)+\gamma_{x}\left(\epsilon_{x}\right)+\gamma_{\theta}\left(\epsilon_{\theta}\right)+\delta_{x} .
\end{gathered}
$$

It remains to prove that (5.50) holds with sufficiently small $\epsilon_{x}, \epsilon_{\theta}>0$, so that $\gamma_{x}\left(\epsilon_{x}\right)+\gamma_{\theta}\left(\epsilon_{\theta}\right) \leq \epsilon_{s}$. To this end, consider the evolution of function

$$
V_{h}^{o}(i):=V_{h}^{o}\left(x_{s}\left(i-T+T_{f}\right), \hat{x}_{s, h}(i-T), \theta_{s}, \hat{\theta}(i)\right)
$$

along the trajectories of the system (5.32), (5.43) for $i \in\left\{T, \ldots, t_{1}\right\}$. One can write that

$\left.\Delta V_{h}^{o}(i)\right|_{(5.32),(5.43)} \leq\left|V_{h}^{o}(i+1)\right|_{(5.32),(5.43)}-\left.V_{h}^{o}(i+1)\right|_{(5.33),(5.41)}\left|+\Delta V_{h}^{o}(i)\right|_{(5.33),(5.41)}$, where $\left.\Delta V_{h}^{o}(i)\right|_{(5.32),(5.43)}:=\left.V_{h}^{o}(i+1)\right|_{(5.32),(5.43)}-V_{h}^{o}(i)$ and $\left.\Delta V_{h}^{o}(i)\right|_{(5.33),(5.41)}:=\left.V_{h}^{o}(i+1)\right|_{(5.33),(5.41)}-V_{h}^{o}(i)$ are variations of $V_{h}^{o}$ along the trajectories of (5.32), (5.43) and (5.33), (5.41), respectively. More precisely,

$$
\begin{gathered}
\left.V_{h}^{o}(i+1)\right|_{(5.32),(5.43)}:=V_{h}^{o}\left(F_{s}^{+}\left(x_{s}\left(i-T+T_{f}\right), u_{s}\left(i-T+T_{f}\right), \theta_{s}\right),\right. \\
f_{s, h}^{+}\left(\hat{x}_{s, h}(i-T), y_{m}(i-T), \hat{\theta}(i-T)\right)+\gamma_{\hat{x}}\left(\hat{x}_{s, h}(i-T), \hat{\theta}(i-T), y_{m}(i-T), \hat{y}_{s}(i)\right), \\
\left.\theta_{s}, \hat{\theta}(i-T)+\gamma_{\hat{\theta}}\left(\hat{x}_{s, h}(i-T), \hat{\theta}(i-T), y_{m}(i-T), \hat{y}_{s}(i)\right)\right),
\end{gathered}
$$


and

$$
\begin{gathered}
\left.V_{h}^{o}(i+1)\right|_{(5.33),(5,41)}:=V_{h}^{o}\left(f_{s, h}^{+}\left(x_{s}\left(i-T+T_{f}\right), u_{s}\left(i-T+T_{f}\right), \theta_{s}\right),\right. \\
f_{s, h}^{+}\left(\hat{x}_{s, h}(i-T), u_{s}\left(i-T+T_{f}\right), \hat{\theta}(i-T)\right) \\
+\gamma_{\hat{x}}\left(\hat{x}_{s, h}(i-T), \hat{\theta}(i-T), u_{s}\left(i-T+T_{f}\right), y_{s}\left(i-T+T_{f}\right)\right), \\
\left.\theta_{s}, \hat{\theta}(i-T)+\gamma_{\hat{\theta}}\left(\hat{x}_{s, h}(i-T), \hat{\theta}(i-T), u_{s}\left(i-T+T_{f}\right), y_{s}\left(i-T+T_{f}\right)\right)\right) .
\end{gathered}
$$

According to Assumption 13, functions $\gamma_{\hat{x}}(\cdot), \gamma_{\hat{\theta}}(\cdot)$ can be chosen such that

$$
\left.\Delta V_{h}^{o}(i)\right|_{(5.33),(5.41)} \leq-\alpha_{3}^{o}\left(V_{h}^{o}(i)\right)
$$

holds, where $\alpha_{3}^{o} \in \mathcal{K}$ is "arbitrarily close" to $\mathbb{I}(\cdot)$ (although $\alpha_{3}^{o}(s)<s$ for all $s>0$ ). On the other hand, by assumption of the lemma, all signals are uniformly bounded on $\left\{0, \ldots, t_{1}\right\}$, therefore Assumptions 12,14 together with continuity of $V_{h}^{o}(\cdot), \gamma_{\hat{x}}(\cdot)$, $\gamma_{\hat{\theta}}(\cdot)$ imply that

$$
\left|V_{h}^{o}(i+1)\right|_{(5.32),(5.43)}-\left.V_{h}^{o}(i+1)\right|_{(5.33),(5.41)} \mid \leq \gamma_{1}(h)+\gamma_{2}\left(\delta_{m}\right)+\gamma_{3}\left(\delta_{s}\right)
$$

for some $\gamma, \gamma_{2}, \gamma_{3} \in \mathcal{K}$ (that possibly depend on $\left.\gamma_{\hat{x}}(\cdot), \gamma_{\hat{\theta}}(\cdot)\right)$. Combining all the above, we see that

$$
\left.\Delta V_{h}^{o}(i)\right|_{(5.32)_{,}(5.43)} \leq-\alpha_{3}^{o}\left(V_{h}^{o}(i)\right)+\gamma_{1}(h)+\gamma_{2}\left(\delta_{m}\right)+\gamma_{3}\left(\delta_{s}\right)
$$

holds for all $i \in\left\{T, \ldots, t_{1}\right\}$. In the above inequality, the possibility to choose $\alpha_{3}^{o}(\cdot) \in \mathcal{K}$ "sufficiently close" to identity function $\mathbb{I} d(\cdot)$ implies that the arbitrarily fast decaying of $V_{h}^{o}(i)$ can be achieved, while sufficiently small $h, \delta_{m}, \delta_{s}>0$ imply that the limit set for $V_{h}^{o}(i)$ can be made arbitrarily small. In particular, given $\epsilon_{v}>0$, the inequality

$$
V_{h}^{o}(i) \leq \epsilon_{v} \quad \text { for all } i \in\left\{T+T_{o}, \ldots, t_{1}+1\right\}
$$

can be guaranteed by an appropriate choice of $\alpha_{3}^{o}(\cdot) \in \mathcal{K}, h, \delta_{m}, \delta_{s}>0$. Choosing $\epsilon_{v}>0$ such that $\epsilon_{v} \leq \alpha_{1}^{o}\left(\min \left\{\epsilon_{x}, \epsilon_{\theta}\right\}\right)$, and taking into account $\tilde{x}_{s, h}\left(t_{1}-T\right)=$ $\hat{x}_{s, h}\left(t_{1}-T\right)$, we see that (5.53) implies (5.50). This completes the proof. 
Lemma 4. Given $\Delta_{x}^{*}>0, t_{1} \geq T+T_{o}$, suppose

$$
\max \left\{\left|x_{m}(i)\right|,\left|x_{s}\left(i+T_{f}\right)\right|,\left|x_{s, h}(i)\right|\right\} \leq \Delta_{x}^{*} \text { for all } i \in\left\{0, \ldots, t_{1}\right\}
$$

Then

$$
\begin{aligned}
& \Delta V_{h}\left(x_{m}\left(t_{1}\right), x_{s}\left(t_{1}+T_{f}\right)\right) \leq-\alpha_{3} \circ[\mathbb{I}-\epsilon]\left(V_{h}\left(x_{m}\left(t_{1}\right), x_{s}\left(t_{1}+T_{f}\right)\right)\right) \\
+ & \hat{\alpha}_{3}\left(\max \left\{\left|x_{s, h}\left(t_{1}\right)-x_{s}\left(t_{1}+T_{f}\right)\right|,\left|x_{s, h}\left(t_{1}+1\right)-x_{s}\left(t_{1}+T_{f}+1\right)\right|\right\}\right) .
\end{aligned}
$$

Proof of Lemma 3. First, condition (5.54) together with Assumption 11 imply directly that $V_{h}\left(x_{m}(i), x_{s}\left(i+T_{f}\right)\right) \leq \alpha_{2}\left(\Delta_{x}^{*}\right)$. Also, by continuity, condition (5.54) implies that all signals (such as $u_{m}, u_{s}, u_{s, h}$, and $u_{H}$ ) are uniformly bounded at time $t_{1}$, therefore $\left|x_{m}\left(t_{1}+1\right)\right|,\left|x_{s}\left(t_{1}+T_{f}+1\right)\right|$ are uniformly bounded, and so is $V_{h}\left(x_{m}(i+1), x_{s}\left(i+T_{f}+1\right)\right)$. The uniform boundedness together with continuity of $V_{h}(\cdot)$ imply that there exists $\gamma_{V} \in \mathcal{K}$ such that

$$
\begin{gathered}
\left|V_{h}\left(x_{m}(i+1), x_{s, h}(i+1)\right)-V_{h}\left(x_{m}(i+1), x_{s}\left(i+T_{f}+1\right)\right)\right| \\
\leq \gamma_{V}\left(\left|x_{s, h}\left(t_{1}+1\right)-x_{s}\left(t_{1}+T_{f}+1\right)\right|\right)
\end{gathered}
$$

and

$$
\left|V_{h}\left(x_{m}(i), x_{s, h}(i)\right)-V_{h}\left(x_{m}(i), x_{s}\left(i+T_{f}\right)\right)\right| \leq \gamma_{V}\left(\left|x_{s, h}\left(t_{1}\right)-x_{s}\left(t_{1}+T_{f}\right)\right|\right) .
$$

Now, we have

$$
\begin{gathered}
\Delta V_{h}\left(x_{m}(i), x_{s}\left(i+T_{f}\right)\right)-\left|V_{h}\left(x_{m}(i), x_{s, h}(i)\right)-V_{h}\left(x_{m}(i), x_{s}\left(i+T_{f}\right)\right)\right| \\
-\left|V_{h}\left(x_{m}(i+1), x_{s, h}(i+1)\right)-V_{h}\left(x_{m}(i+1), x_{s}\left(i+T_{f}+1\right)\right)\right| \\
\leq \Delta V_{h}\left(x_{m}(i), x_{s, h}(i)\right) \\
\leq-\alpha_{3}\left(V_{h}\left(x_{m}(i), x_{s, h}(i)\right)-V_{h}\left(x_{m}(i), x_{s}\left(i+T_{f}\right)\right)+V_{h}\left(x_{m}(i), x_{s}\left(i+T_{f}\right)\right)\right) \\
\leq-\alpha_{3} \circ[\mathbb{I}-\epsilon]\left(V_{h}\left(x_{m}(i), x_{s}\left(i+T_{f}\right)\right)\right) \\
+\alpha_{3} \circ\left[\epsilon^{-1}+\mathbb{I}\right]\left(\left|V_{h}\left(x_{m}(i), x_{s, h}(i)\right)-V_{h}\left(x_{m}(i), x_{s}\left(i+T_{f}\right)\right)\right|\right) .
\end{gathered}
$$


Substituting (5.56), (5.57) into (5.58) and performing simple calculations, one gets (5.55).

Now the proof of Theorem 9 can be completed as follows. Suppose (5.45) holds for some $\Delta_{x}>0$. Applying Lemma 2, we get (5.47); In particular, inequality

$$
V_{h}\left(t_{1}\right) \leq \alpha_{2} \circ \beta_{1}\left(\Delta_{x}\right)
$$

holds for $t_{1}=T+T_{0}$. Choosing $\Delta_{x}^{*}:=\alpha_{1}^{-1} \circ \alpha_{2} \circ \beta_{1}\left(\Delta_{x}\right)+a$, where $a>0$, and applying Lemma 2, we see that (5.48) holds for $t_{1}=T+T_{0}$. Applying Lemmas 3, 4, and combining their results, we see that the inequality

$$
\begin{aligned}
\Delta V_{h}\left(x_{m}\left(t_{1}\right), x_{s}\left(t_{1}+T_{f}\right)\right) \leq & -\alpha_{3} \circ[\mathbb{I}-\epsilon]\left(V_{h}\left(x_{m}\left(t_{1}\right), x_{s}\left(t_{1}+T_{f}\right)\right)\right) \\
& +\hat{\alpha}_{3}\left(\rho_{h}^{*}(h)+\gamma_{\delta s}\left(\delta_{s}\right)+\gamma_{\delta m}\left(\delta_{m}\right)+\delta_{x}+\epsilon_{s}\right)
\end{aligned}
$$

is valid for $t_{1}=T+T_{\text {o }}$.

Now suppose all $h, \delta_{s}, \delta_{m}, \delta_{x}, \epsilon_{s}>0$ are sufficiently small such that

$$
\alpha_{3} \circ[\mathbb{I}-\epsilon] \circ \alpha_{2} \circ \beta_{1}\left(\Delta_{x}\right) \geq 2 \hat{\alpha}_{3}\left(\rho_{h}^{*}(h)+\gamma_{\delta s}\left(\delta_{s}\right)+\gamma_{\delta m}\left(\delta_{m}\right)+\delta_{x}+\epsilon_{s}\right)
$$

and

$$
\rho_{h}^{*}(h)+\gamma_{\delta s}\left(\delta_{s}\right)+\gamma_{\delta m}\left(\delta_{m}\right)+\delta_{x}+\epsilon_{s} \leq a .
$$

Combination of (5.60), (5.61) with the fact that (5.59) holds for $t_{1}=T+T_{0}$ implies that the last holds also for $t_{1}=T+T_{o}+1$, and $\max \left\{\left|x_{m}\left(t_{1}+1\right)\right|,\left|x_{s}\left(t_{1}+1+T_{f}\right)\right|\right\} \leq$ $\alpha_{1}^{-1} \circ \alpha_{2} \circ \beta_{1}\left(\Delta_{x}\right)$. On the other hand, taking into account (5.62) and applying Lemma 3 , one gets $\left|x_{s, h}\left(t_{1}+1\right)-x_{s}\left(t_{1}+T_{f}+1\right)\right| \leq a$. Combining the last two inequalities, we see that (5.48) holds for $t_{1}=T+T_{o}+1$, and therefore (5.60) also holds for $t_{1}=T+T_{o}+1$, etc. Continuing by induction arguments, one gets that (5.60) holds for all $t_{1} \geq T+T_{0}$. Now, choosing $a>0$ sufficiently small, applying Lemma 1, and taking into account (5.49), (5.62), one can obtain (5.46). The proof of Theorem 9 is complete. 


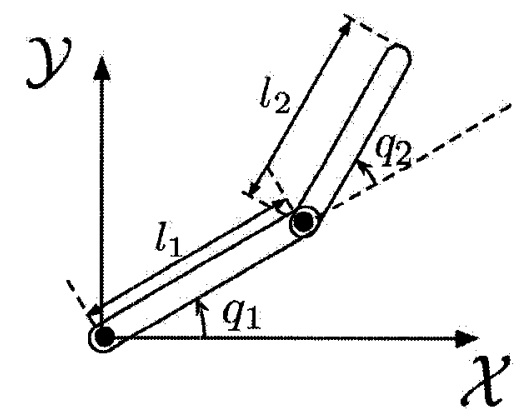

Figure 5.2: Kinematic Scheme

\subsection{Example}

We consider a teleoperator system where the master and the slave devices are identical two-degrees-of-freedom planar manipulators shown in figure 5.2. The forward kinematics of the master and the slave devices are described as follows

$$
\begin{aligned}
& x=l_{1} \cos q_{1}+l_{2} \cos \left(q_{1}+q_{2}\right), \\
& y=l_{1} \sin q_{1}+l_{2} \sin \left(q_{1}+q_{2}\right),
\end{aligned}
$$

where $x, y$ are the Cartesian coordinates, $q_{1}, q_{2}$ are joint angles, and $l_{1}=0.7 \mathrm{~m}$, $l_{2}=0.5 \mathrm{~m}$. The dynamics of both the master $(i=m)$ and the slave $(i=s)$ manipulators are described by Euler-Lagrange equations with compensated gravity terms, as follows

$$
H_{i}\left(q_{i}\right) \ddot{q}_{i}+C_{i}\left(q_{i}, \dot{q}_{i}\right) \dot{q}_{i}=\tau_{i}
$$

where

$$
\begin{gathered}
H(q)=\left[\begin{array}{cc}
\left(2 l_{1} \cos q_{2}+l_{2}\right) l_{2} m_{2}+l_{1}^{2}\left(m_{1}+m_{2}\right) & l_{2}^{2} m_{2}+l_{1} l_{2} m_{2} \cos q_{2} \\
l_{2}^{2} m_{2}+l_{1} l_{2} m_{2} \cos q_{2} & l_{2}^{2} m_{2}
\end{array}\right], \\
C(q, \dot{q})=\left[\begin{array}{cc}
-l_{1} l_{2} m_{2} \sin \left(q_{2}\right) \dot{q}_{2} & -l_{1} l_{2} m_{2} \sin \left(q_{2}\right)\left(\dot{q}_{1}+\dot{q}_{2}\right) \\
l_{1} l_{2} m_{2} \sin \left(q_{2}\right) \dot{q}_{1} & 0
\end{array}\right]
\end{gathered}
$$


and the masses are $m_{1}=10 \mathrm{~kg}$, and $m_{2}=5 \mathrm{~kg}$. Differentiating (5.63) with respect to time, we get the expression for spatial velocities, as follows

$$
\begin{aligned}
& \dot{x}=l_{1}\left(-\sin \left(q_{1}\right)\right) \dot{q}_{1}+l_{2}\left(-\sin \left(q_{1}+q_{2}\right)\right)\left(\dot{q}_{1}+\dot{q}_{2}\right), \\
& \dot{y}=l_{1} \cos \left(q_{1}\right) \dot{q}_{1}+l_{2} \cos \left(q_{1}+q_{2}\right)\left(\dot{q}_{1}+\dot{q}_{2}\right),
\end{aligned}
$$

or

$$
\left[\begin{array}{c}
\dot{x} \\
\dot{y}
\end{array}\right]=J_{s}(q)\left[\begin{array}{l}
\dot{q}_{1} \\
\dot{q}_{2}
\end{array}\right]
$$

where the spatial jacobian is

$$
J_{s}(q)=\left[\begin{array}{cc}
-l_{1} \sin \left(q_{1}\right)-l_{2} \sin \left(q_{1}+q_{2}\right) & -l_{2} \sin \left(q_{1}+q_{2}\right) \\
l_{1} \cos \left(q_{1}\right)+l_{2} \cos \left(q_{1}+q_{2}\right) & l_{2} \cos \left(q_{1}+q_{2}\right)
\end{array}\right] .
$$

Spatial acceleration of the end-effector is therefore given by the formula

$$
\left[\begin{array}{l}
\ddot{x} \\
\ddot{y}
\end{array}\right]=J_{s}(q)\left[\begin{array}{l}
\ddot{q}_{1} \\
\ddot{q}_{2}
\end{array}\right]+\dot{J}_{s}(q, \dot{q})\left[\begin{array}{l}
\dot{q}_{1} \\
\dot{q}_{2}
\end{array}\right],
$$

where

$$
\dot{J}_{s}(q, \dot{q}):=\left[\begin{array}{cc}
-l_{1} \cos \left(q_{1}\right) \dot{q}_{1}-l_{2} \cos \left(q_{1}+q_{2}\right)\left(\dot{q}_{1}+\dot{q}_{2}\right) & -l_{2} \cos \left(q_{1}+q_{2}\right)\left(\dot{q}_{1}+\dot{q}_{2}\right) \\
-l_{1} \sin \left(q_{1}\right) \dot{q}_{1}-l_{2} \sin \left(q_{1}+q_{2}\right)\left(\dot{q}_{1}+\dot{q}_{2}\right) & -l_{2} \sin \left(q_{1}+q_{2}\right)\left(\dot{q}_{1}+\dot{q}_{2}\right)
\end{array}\right] .
$$

The input torque of the master manipulator is described by the formula

$$
\tau_{m}=J_{s}\left(q_{m}\right)\left(F_{h}-\hat{F}_{r}\right)
$$

where $F_{h}$ is the forces applied by the human operator, and $\hat{F}_{r}$ is the force reflection term. The human operator attempts to move the master along the reference trajectory $\left(x_{r e f}(t), y_{r e f}(t)\right)$ shown in figure 5.3. More precisely, the forces exerted by the human operator are described as follows

$$
F_{h}=K_{\text {hum }}\left(\left[\begin{array}{c}
\dot{x}_{r e f}(t) \\
\dot{y}_{r e f}(t)
\end{array}\right]-\left[\begin{array}{c}
\dot{x}_{m}(t) \\
\dot{y}_{m}(t)
\end{array}\right]+\Lambda_{\text {hum }}\left(\left[\begin{array}{l}
x_{r e f}(t) \\
y_{r e f}(t)
\end{array}\right]-\left[\begin{array}{l}
x_{m}(t) \\
y_{m}(t)
\end{array}\right]\right)\right)
$$

where $K_{\text {hum }}=40$ and $\Lambda_{\text {hum }}=10$; thus, the human operator is modeled as a PD controller with P-gain equal to $K_{\text {hum }} \Lambda_{\text {hum }}$ and D-gain equal to $K_{h u m}$. 

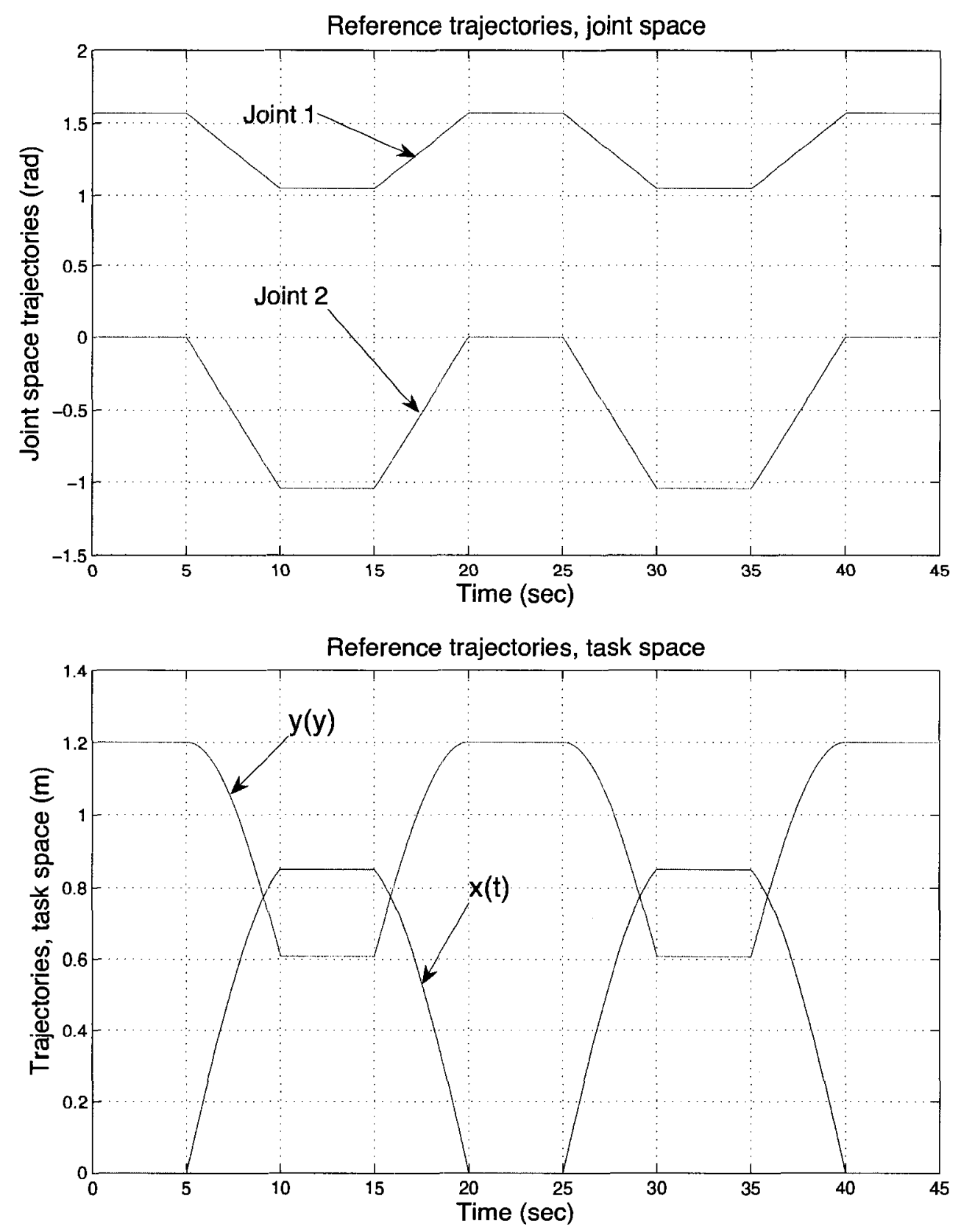

Figure 5.3: Reference trajectories: joint space (top) and task space(bottom) 
On the other hand, the input torque of the slave manipulator is described according to the following equation

$$
\tau_{s}=u_{s}-J_{s}^{T}\left(q_{s}\right) f_{i n t},
$$

where $u_{s}$ is the local control torque and $f_{\text {int }}$ is the interaction forces between the slave and the environment. In these simulations, we assume that the end-effector of the slave manipulator is permanently coupled with the environment; the last is described as a mass-spring-damper system, according to the formula

$$
m \ddot{x}_{s}+b \dot{x}_{s}+k x_{s}=f_{i n t}
$$

where $x_{s}$ is the position of the slave's end-effector, $f_{\text {int }}$ is the interaction force between the slave and the environment, $m>0, b>0$, and $k>0$ are environmental inertia (mass), damping and stiffness coefficients, respectively. In the simulations below, the numerical values of these parameters are chosen to be $m=80, b=120$, $k=150$; these values, however, are assumed to be unknown and, therefore, are not utilized directly in the control laws. The local slave controller is essentially a passivity-based tracking controller that attempts to track the delayed master position $\hat{q}_{m}(t)=q_{m}\left(t-T_{f}\right)$; it consists of the filter

$$
\begin{gathered}
\dot{\xi}_{1}=\xi_{2}+g \alpha_{1}\left(\hat{q}_{m}-\xi_{1}\right), \\
\dot{\xi}_{2}=g^{2} \alpha_{0}\left(\hat{q}_{m}-\xi_{1}\right),
\end{gathered}
$$

and the control law

$$
\begin{aligned}
u_{s}= & H_{s}\left(q_{s}\right)\left(\dot{\xi}_{2}+\Lambda_{s}\left(\dot{\xi}_{1}-\dot{q}_{s}\right)\right)+C_{s}\left(q_{s}, \dot{q}_{s}\right)\left(\xi_{2}+\Lambda_{s}\left(\xi_{1}-q_{s}\right)\right) \\
& +K_{s}\left(\dot{q}_{s}-\xi_{2}+\Lambda_{s}\left(q_{s}-\xi_{1}\right)\right),
\end{aligned}
$$

where the parameters are $\alpha_{0}=2, \alpha_{1}=3, g=10, K_{s}=100$, and $\Lambda_{s}=10$.

In the simulations below, we compare the performance of the direct force reflection scheme with a virtual environment based scheme which is designed according 


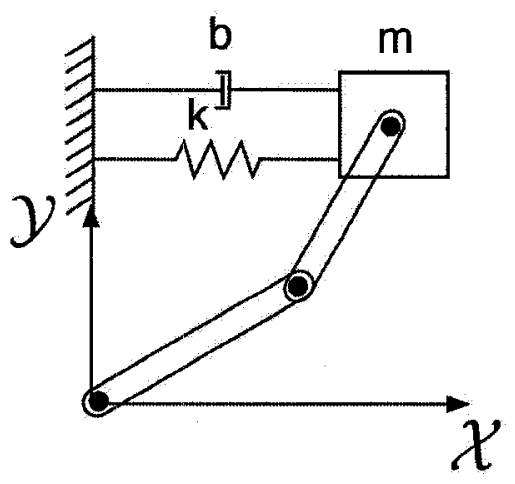

Figure 5.4: Slave manipulator interconnected with environment

to the methodology proposed in this Chapter. In the case of direct force reflection, the force reflection term on the master side is the delayed version of the interaction forces between the slave and the environment, i.e.,

$$
\hat{F}_{r}(t)=f_{\text {int }}\left(t-T_{b}\right),
$$

where $T_{b}$ is the communication delay in the backward (from slave to master) channel. In the simulations below, we choose $T_{f}=T_{b}=0.25 \mathrm{sec}$, which makes the round trip time delay equal to $0.5 \mathrm{sec}$. The results of simulation is illustrated by the plot presented in figure 5.4, where the x-trajectories of the master and the slave are shown. One can see that even existence of relatively small delays makes the system's response clearly unstable.

Next, performance of a virtual environment based teleoperator scheme proposed in this work was addressed. In this set of simulations, the virtual environment on the master side is represented by an approximate discrete-time model of the remote slave+environment interconnection, more precisely, by its Euler approximate model with integration step equal to $0.005 \mathrm{sec}$. Since the parameters of the remote environment are unknown, a recursive least-squares identification algorithm is implemented that provides estimates of the remote environment parameters based on 


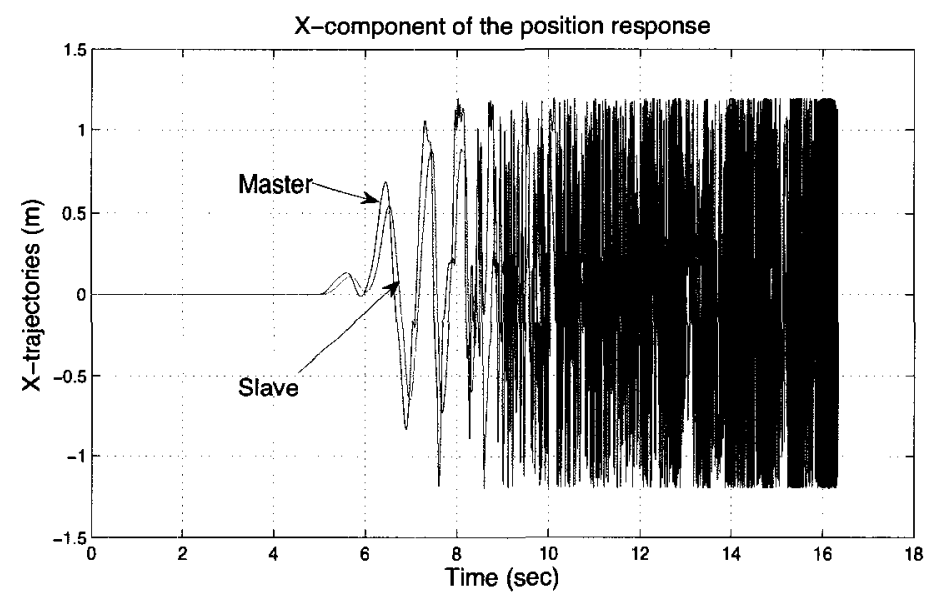

Figure 5.5: Direct force feedback: X-trajectories, master and slave devices

delayed data. To derive this algorithm, let us first rewrite the system (5.68) in the form

$$
\ddot{x}_{s}=-\theta_{2} \dot{x}_{s}-\theta_{1} x_{s}+\theta_{3} f_{i n t},
$$

where $\theta_{1}=k / m, \theta_{2}=b / m$, and $\theta_{3}=1 / m$. The discrete-time Euler approximate model for the system (5.68) can be written in the form

$$
y(i)=\phi(i) \theta
$$

where $\phi(i)=\left[-x_{s}(i-2) ; T^{-1}\left(x_{s}(i-2)-x_{s}(i-1)\right) ; f_{\text {int }}(i-2)\right], \theta=\left[\theta_{1}, \theta_{2}, \theta_{3}\right]^{T}$, $y(i)=T^{-2}\left(x_{s}(i)-x_{s}(i-2)+2 x_{s}(i-1)\right)$, and $T>0$ is sampling period. For the model (5.71), a recursive least squares identification algorithm can be derived according to the following formula

$$
\hat{\theta}(i+1)=\hat{\theta}(i)+K(i+1)(y(i+1)-\phi(i+1) \hat{\theta}(i)),
$$

where $K(i+1)=P(i) \phi^{T}(i+1)\left(1+\phi(i+1) P(i) \phi^{T}(i+1)\right)^{-1}$, and $P(\cdot)$ is a solution of the recursive matrix equation

$$
P(i+1)=(\mathbb{I}-K(i+1) \phi(i+1)) P(i)
$$


with "sufficiently large" initial condition $P(0)$. In the simulations presented below, $P(0)=\operatorname{diag}\{1000,1000,1000\}$. The results of simulation of the virtual environment based teleoperator system is illustrated by the plots presented in figure 5.6. In these plots, the position response of the master, slave, and virtual slave are presented, as well as the corresponding force responses; in these plots, in order to demonstrate more explicitly the position and force tracking properties of the scheme, the response of the slave is shifted ahead in time to the amount of one-way communication delay, i.e., $0.25 \mathrm{sec}$. One can see that the overall system is perfectly stable, and, after some transient time, both the position and the force response of the virtual slave track closely the corresponding trajectories of the actual slave+environment interconnection. Thus, one can see that the proposed scheme, besides improving transparency by providing the human operator with the essential position/force information without communication delay, also restores stability properties of the overall force reflecting teleoperator system.

\subsection{Conclusions}

In this chapter, we present a model-based approach to the design of VR-enhanced network-based force-reflecting teleoperators. The proposed method is built on sampleddata stabilization of a nonlinear system based on its approximate discrete-time model. A protocol for control and communication between the plant and the controller nodes is proposed that copes with irregularity of the transfer intervals, existence of time-varying communication delays, possibility of packet losses, and absence of clock synchronization between the nodes. For a general nonlinear system, stability plus performance recovery result is proven. It is shown that the maximum allowable transfer interval can be assigned arbitrarily by an appropriate choice of the mod- 

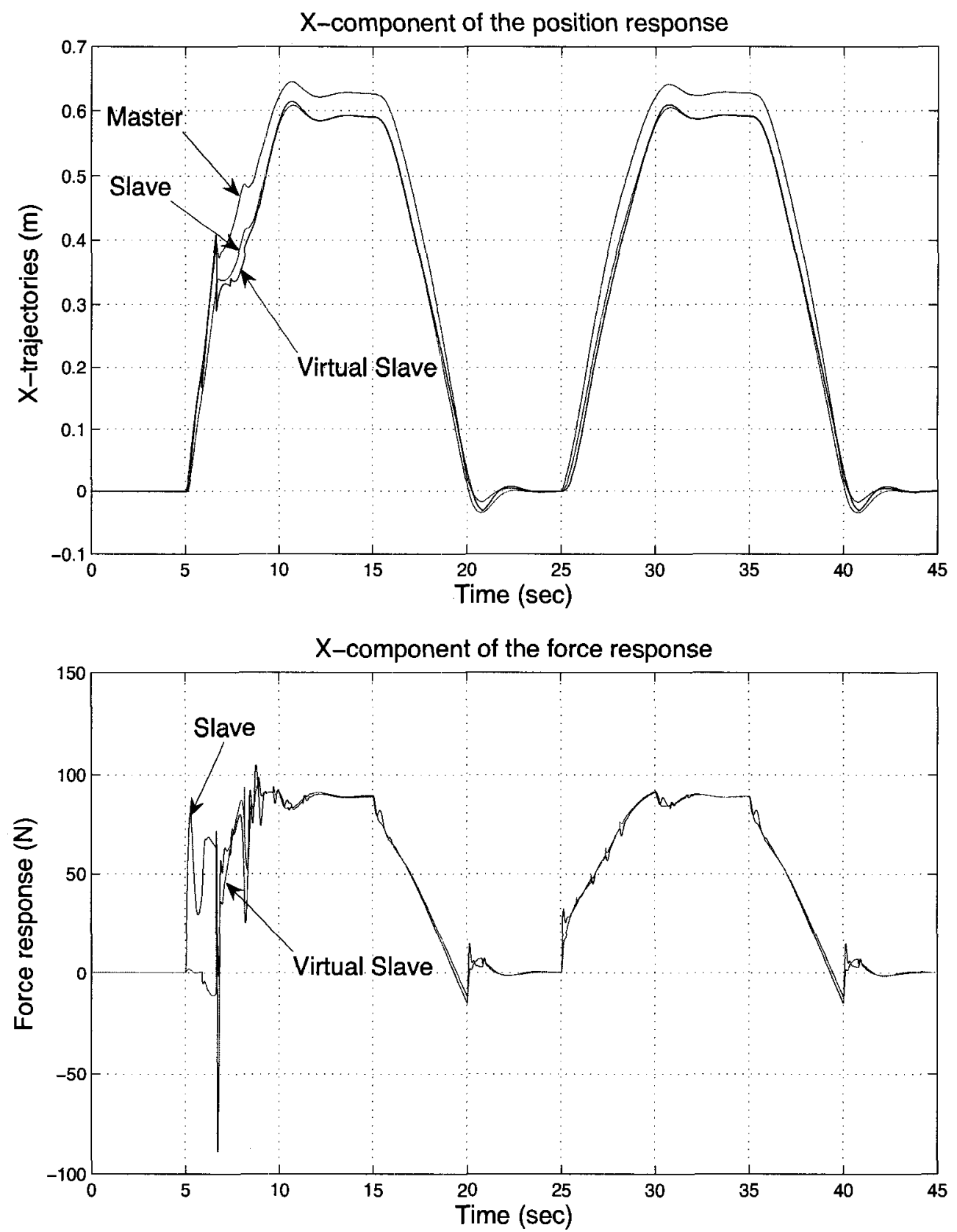

Figure 5.6: VE based force feedback: X-trajectories, master, slave and virtual slave (top); X-components of the contact forces, environment and virtual environment (bottom) 
elling parameter, horizon of control prediction as well as buffer size. This method is then applied to the design of virtual reality enhanced network-based bilateral teleoperator system. More precisely, it is assumed that a set of partially known approximate discrete-time models of the remote dynamics is available to the designer. These approximate models are used for design of a virtual environment block that incorporates state estimation/parameter identification of the remote dynamics together with an approximate trajectory prediction algorithms. Thus designed VE block provides the human operator with a local force feedback that is not subject to communication delay/jitter; therefore, it restores the sense of causality for the human operator and does not have destabilizing effect of the delayed force feedback. A simulation example is presented that demonstrates improvernent of the stability/transparency properties of the teleoperator system achieved by using the proposed method. 


\section{Chapter 6}

\section{Concluding Remarks}

\subsection{Thesis Contributions}

In this thesis, a set of results is developed that is directed towards achieving stable and transparent force-reflecting teleoperation in the presence of communication constraints typical for communication networks. One of the major goals of this thesis is to advocate the use of small-gain approach to the design of the networkbased force reflecting teleoperators. The traditional view on using the small gain approach in teleoperation tasks is somewhat skeptical [15]. The major part of this skepticism comes from belief that the small gain approach results in conservative design leading to poor transparency due to limited force reflection gain. In our opinion, the set of results presented in this work substantially challenges this point of view. Indeed, the use of projection-based force reflection algorithms proposed in this thesis effectively removes the constraints on force reflection gain imposed by the small-gain requirement. It is worth noting that, once these constraints are

removed, the small gain approach becomes very effective particularly in the case of network based teleoperation, due to the fact that it imposes virtually no restrictions 
on the characteristics of communication delay. On the contrary, the more popular passivity-based approaches generally impose significant restrictions on communication process, due to the fact that irregularity of the communication delays and the information losses may result in generation of energy in the communication channel, which destroys the overall passivity and leads to instability. There are a number of other limitations typical for passivity-based approaches (such as problems with position tracking, the requirement of equal number of inputs and outputs for each subsystems, etc.) that are absent in the case of small-gain design. Overall, the results presented in Chapters $2-4$ of this thesis may significantly increase applicability of the small-gain approach to network-based teleoperation tasks.

Another set of the results presented in this thesis is related to the design of network-based teleoperators enhanced by means of a virtual environment block implemented on the master side. The virtual environment block runs the remote state estimation, identification, and trajectory prediction algorithms that are based on an available model of the remote process and delayed possibly corrupted measurements. This is a scheme alternative to the direct force feedback which may help to restore the sense of causality for the human operator and to guarantee stability of the overall system in the presence of significant communication constraints. Our approach is based on certain results from sampled-data nonlinear systems design based on partial knowledge of a set of approximate discrete-time models of the remote system. An essential and distinct feature of our approach is the simultaneous design of a control algorithm and a communication protocol that together guarantee the overall scheme stability and performance. The framework proposed may lead to significant improvement of performance in virtual reality based teleoperation over communication networks. 


\subsection{Manuscripts Published and Submitted}

The research presented in this thesis resulted in a number of refereed publications, both in journals and conference proceedings. The list of publications with brief description of contribution is given below. First, the multichannel IOS small-gain theorem for systems with network-induced communication constraints presented in Chapter 2 of this thesis was also the main topic of the following paper:

1. I. G. Polushin, H. J. Marquez, A. Tayebi, and P. X. Liu "A Multichannel IOS Small Gain Theorem for Systems with Multiple Time-Varying Communication Delays", IEEE Transactions on Automatic Control, 2009, Vol. 54, No. 1, to appear; Reference [98].

See also the preliminary (conference) version of the above paper, as follows:

2. I. G. Polushin, H. J. Marquez, A. Tayebi, and P. X. Liu "A Multichannel IOS Small Gain Theorem for Systems with Multiple Time-Varying Communication Delays," 46th IEEE Conference on Decision and Control, New Orleans, LA, December 12-14, 2007, pp. 3853 - 3858; Reference [99].

The small-gain based schemes for bilateral teleoperation over communication networks (presented in Chapter 3 of this Thesis) are addressed in the following articles:

3. I. G. Polushin, P. X. Liu, and C.-H. Lung "A Control Scheme for Stable ForceReflecting Teleoperation over IP Networks", IEEE Transactions on Systems, Man and Cybernetics, Part B: Cybernetics, 2006, Vol. 36, No. 4, pp. $930-$ 939; Reference [60].

4. I. G. Polushin, P. X. Liu, C.-H, Lung, and G.D. On "Position-Error Based Schemes for Bilateral Teleoperation with Time Delay: Theory and Experi- 
ments", ASME Journal of Dynamic Systems, Measurement and Control, provisionally accepted.

The preliminary versions of the above two papers were also published in refereed conferences proceedings, as follows:

5. I. G. Polushin, P. X. Liu, and C.-H. Lung "A Control Scheme for Stable Force-Reflecting Teleoperation over IP Networks", IEEE/RSJ International Conference on Intelligent Robots and Systems IROS 2005, Edmonton, AB, August 2005, pp. 2731- 2736; Reference [100].

6. I. G. Polushin, P. X. Liu, and C.-H. Lung "Position-Error Based Schemes for Bilateral Teleoperation with Time Delay: Theory and Experiments", International Conference on Mechatronics and Automation ICMA 2006, R. P. China, June 2006, pp. 312 - 317. The paper received Best Conference Paper Award. Reference [101].

Projection-based force reflection algorithms (Chapter 4) were the main topic of the following publications:

7. I. G. Polushin, P. X. Liu, and C.-H. Lung "A Force Reflection Algorithm for Improved Transparency in Bilateral Teleoperation with Communication Delay", IEEE/ASME Transactions on Mechatronics, 2007, Vol. 12, No. 3, pp. 361 - 374; Reference [102].

8. I. G. Polushin, P. X. Liu, and C.-H. Lung "Projection-Based Force Reflection Algorithm for Stable Bilateral Teleoperation over Networks", IEEE Transactions on Instrumentation and Measurement, 2008, Vol. 57, No. 9, pp. 1854 1865; Reference [103]. 
Also, the general stability result for teleoperator systems with projection-based force reflection algorithms presented in Section 4.4 of this Thesis, is the main topic of the following paper which is currently under review:

9. I. G. Polushin, P. X. Liu, and C.-H. Lung "Stability of Bilateral Teleoperators with Projection-Based Force Reflection Algorithms," IEEE Transactions on Robotics, in review.

The preliminary versions of the above papers were published in refereed conferences proceedings, as follows.

10. I. G. Polushin, P. X. Liu, and C.-H. Lung "A Force Reflection Algorithm for Improved Transparency in Bilateral Teleoperation with Communication Delay", International Conference on Robotics and Automation ICRA 2006, Orlando, FL, May 2006, pp. 2914 - 2920; Reference [104].

11. I. G. Polushin, P. X. Liu, and C.-H. Lung "Projection-Based Force Reflection Algorithm for Stable Bilateral Teleoperation over Networks," 2007 IEEE/RSJ International Conference on Intelligent Robots and Systems, San Diego, CA, October 29 - November 2, 2007, pp. 2654 - 2659; Reference [105].

12. I. G. Polushin, P. X. Liu, and C.-H. Lung "Stability of Bilateral Teleoperators with Projection-Based Force Reflection Algorithms," International Conference on Robotics and Automation ICRA 2008, Pasadena, CA, May 2008, pp. 677 - 682; Reference [106].

The model-based approach to control of nonlinear systems over networks as well as the application of this approach to the design of virtual environment enhanced teleoperator systems (Chapter 5 of this Thesis) were addressed in the following papers: 
13. I. G. Polushin, P. X. Liu, and C.-H. Lung "On the Model-Based Approach to Nonlinear Networked Control Systems", Automatica, 2008, Vol. 44, No. 9, pp. 2409 - 2414; Reference [85].

14. I. G. Polushin, P. X. Liu, and C.-H. Lung "On the Model-Based Approach to Nonlinear Networked Control Systems," 2007 American Control Conference, New York City, NY, July 11-13, 2007, pp. 281 - 286; Reference [107].

15. I. G. Polushin, J. P. Rhinelander, P. X. Liu, and C.-H. Lung "A Scheme for Virtual Reality Enhanced Bilateral Teleoperation with Time Delay," 2008 IEEE Instrumentation \& Measurement Technology Conference, Victoria, BC, May 2008, pp. 1819-1822; Reference [108].

16. I. G. Polushin, J. P. Rhinelander, P. X. Liu, and C.-H. Lung "Virtual Reality Enhanced Bilateral Teleoperation with Communication Constraints," submitted to International Conference on Robotics and Automation ICRA 2009, Kobe, Japan.

There are a number of topics on which the research work is still in progress. Some of these topics are briefly discussed in the next section.

\subsection{Possible Directions for Future Research}

The following are possible topics for the future research.

1. The projection-based force reflecting algorithms proposed in this thesis have their origin in a very simple observation: the force felt by the human is exactly the component of the external force that is compensated by the human hand. A deeper insight into the human force sensing/perception processes can possibly result in more sophisticated and effective force reflection algorithms. An 
example of a conceptually different approach to the design of force-reflecting teleoperators that is also based on certain features of the human force sensing/perception process is the approach adopted in [109] (see also [69]). In these works, the design is based on the observation that the frequency ranges of the manipulation and of the human perception are actually occupy two very distinct frequency bands. As a first step, it would be interesting to compare and possibly combine these two approaches, which may result in design of new and improved force-reflecting algorithms.

2. Although it seems to make a perfect combination with the small-gain approach, the idea of projection-based force reflection appears to be independent on the particular teleoperator design framework. It would be interesting, therefore, to understand how this idea can be incorporated into the teleoperator systems that are designed based on different principles (such as the wave-based approach).

3. Although the small-gain theorem (Theorem 1) presents stability conditions for multichannel network based interconnections, the teleoperation schemes presented in Sections 3, 4, are essentially two-channel schemes (position in the forward channel, force in the backward one). It would be interesting, therefore, to find appropriate extensions of this approach to the case of the four-channel scheme (position/force in both the forward and the backward channels) as well as various three channel schemes.

4. Extensive experimental evaluation of the approach to virtual reality enhanced bilateral teleoperation proposed in Chapter 5 would be highly desirable.

5. The very brief Chapter B can itself be considered as on outline of possible future research related to communication aspects of teleoperation and, in par- 
ticular, telesurgery over the Internet.

6. Applications of the proposed methods to specific problems of (tele)robotic surgery would be of great interest. 


\section{References}

[1] T. B. Sheridan, "Telerobotics," Automatica, vol. 25, no. 4, pp. 487-507, 1989.

[2] J. Marescaux, J. Leroy, M. Gagner, F. Rubino, D. Mutter, M. Vix, S. E. Butner, and M. K. Smith, "Transatlantic robot-assisted telesurgery," Nature, vol. 413, pp. 379-380, 2001.

[3] S. E. Butner and M. Ghodoussi, "Transforming a surgical robot for human telesurgery," IEEE Transactions on Robotics and Automation, vol. 19, pp. 818-824, Oct. 2003.

[4] S. Charles, "Dexterity enhancement for surgery," in Proceedings of the 1st Int. Symp. Medical Robotics and Computer Assisted Surgery, vol. 2, pp. 145-160, 1994.

[5] I. W. Hunter, L. A. Jones, M. A. Sagar, S. R. Lafontaine, and P. J. Hunter, "Ophthalmic microsurgical robot and associated virtual environment," Computers in Biology and Medicine, vol. 25, pp. 173-182, Mar. 1995.

[6] S. E. Salcudean, S. Ku, and G. Bell, "Performance measurement in scaled teleoperation for microsurgery," in Proceedings of the First Joint Conference on Computer Vision, Virtual Reality and Robotics in Medicine and Me- 
dial Robotics and Computer-Assisted Surgery, (London, UK), pp. 789-798, Springer-Verlag, 1997.

[7] M. Misuishi, H. Watanabe, H. Nakanishi, H. Kubota, and Y. IIzuka, "Dexterity enhancement for a tele-microsurgery system with multiple macro-micro colocated operation point manipulators and understanding of the operator's intention," in Proceedings of the 1st Joint Conf. Computer Vision, Virtual Reality and Robotics in Medicine and Medical Robotics and Computer-Assisted Surgery, (Grenoble, France), pp. 821-830, 1997.

[8] P. S. Green, J. W. Hill, J. F. Jensen, and A. S. Shah, "Telepresence surgery," IEEE Engineering in Medicine and Biology, vol. 14, no. 3, pp. 324-329, 1995.

[9] C. R. Wagner, N. Stylopoulos, and R. D. Howe, "The role of force feedback in surgery: Analysis of blunt dissection," in Proceedings of 10th Symposium on Haptic Interfaces for Virtual Environment and Teleoperator Systems HAPTICS 2002, (Orlando, Florida, USA), pp. 68-74, 2002.

[10] G. Tholey, J. P. Desai, and A. E. Castellanos, "Force feedback plays a significant role in minimally invasive surgery: Results and analysis," Annals of Surgery, vol. 241, pp. 102-109, Jan. 2005.

[11] M. Kitagawa, A. Okamura, B. Bethea, V. Gott, and W. Baumgartner, "Analysis of suture manipulation forces for teleoperation with force feedback," in Proceedings of the Fifth International Conference on Medical Image Computing and Computer Assisted Intervention - MICCAI 2002, pp. 155-162, 2002.

[12] A. M. Okamura, "Methods for haptic feedback in teleoperated robot-assisted surgery," Industrial Robot, vol. 31, no. 6, pp. 499-508, 2004. 
[13] G. J. Raju, G. C. Verghese, and T. B. Sheridan, "Design issues in 2-port network models of bilateral remote manipulation," in International Conference on Robotics and Automation, pp. 1316-1321, 1989.

[14] Y. Yokokohji and T. Yoshikawa, "Bilateral control of master-slave manipulators for ideal kinesthetic coupling - formulation and experiment," IEEE Transactions on Robotics and Automation, vol. 10, pp. 605-620, Oct. 1994.

[15] D. A. Lawrence, "Stability and transparency in bilateral teleoperation," IEEE Transactions on Robotics and Automation, vol. 9, pp. 624-637, Oct. 1993.

[16] M. C. Cavusoglu, "Telesurgery and surgical simulation: Design, modelling, and evaluation of haptic interfaces to real and virtual surgical environments." $\mathrm{PhD}$ Thesis, University of California at Berkeley, 2000.

[17] J. E. Colgate, "Robust impedance shaping telemanipulation," IEEE Transactions on Robotics and Automation, vol. 9, pp. 374-384, Aug. 1993.

[18] H. Kazerooni and G. Jenhwa, "Human extenders," Transactions of the ASME Journal of Dynamic Systems, Measurement and Control, vol. 115, pp. 281290, June 1993.

[19] R. Taylor, P. Jensen, L. Whitcomb, R. Kumar, D. Stoianovici, P. Gupta, Z.-X. Wang, E. de Juan, and L. Kavoussi, "A steady-hand robotic system for microsurgical augmentation," International Journal of Robotics Research, vol. 18, pp. 1201-1210, Dec. 1999.

[20] M. C. Cavusoglu, A. Sherman, and F. Tendick, "Design of bilateral teleoperation controllers for haptic exploration and telemanipulation of soft environments," IEEE Transactions on Robotics and Automation, vol. 18, pp. 641-647, Aug. 2002. 
[21] R. J. Anderson and M. W. Spong, "Bilateral control of teleoperators with time delay," IEEE Trans. Aut.Contr., vol. AC-34, pp. 494-501, May 1989.

[22] M. D. Fabrizio, B. R. Lee, D. Y. Chan, D. Stoianovici, T. W. Jarrett, C. Yang, and L. R. Kavoussi, "Effect of time delay on surgical performance during telesurgical manipulation," Journal of endourology, vol. 14, pp. 133-141, Mar. 2000.

[23] K. Hashtrudi-Zaad and S. S. Salcudean, "Transparency in time-delayed systems and the effect of local force feedback for transparent teleoperation," IEEE Transactions on Robotics and Automation, vol. 18, pp. 108-114, Feb. 2002.

[24] G. Niemeyer and J.-J. E. Slotine, "Telemanipulation with time delays," International Journal of Robotics Research, vol. 23, pp. 873-890, Sept. 2004.

[25] W. R. Ferrell, "Delayed force feedback," Human Factors, vol. 8, pp. 449-455, 1966.

[26] G. Niemeyer and J.-J. E. Slotine, "Stable adaptive teleoperation," IEEE Journal of Oceanic Engineering, vol. 16, pp. 152-162, Jan. 1991.

[27] S. Munir and W. J. Book, "Internet-based teleoperation using wave variables with prediction," IEEE/ASME Transactions on Mechatronics, vol. 7, pp. 124133 , June 2002.

[28] S. Stramigioli, A. van der Schaft, B. Maschke, and C. Melchiorri, "Geometric scattering in robotic telemanipulation," IEEE Transactions on Robotics and Automation, vol. 18, pp. 588-596, Aug. 2002.

[29] W. S. Kim, B. Hannaford, and A. K. Bejczy, "Force-reflection and shared com- 
pliant control in operating telemanipulators with time delay," IEEE Transactions on Robotics and Automation, vol. 8, pp. 176-185, Apr, 1992.

[30] G. M. Leung, B. A. Francis, and J. Apkarian, "Bilateral controller for teleoperators with time delay via mu-synthesis," IEEE Trans. Robotics and Automation, vol. 11, pp. 105-116, 1995.

[31] W.-H. Zhu and S. E. Salcudean, "Stability guaranteed teleoperation: an adaptive motion/force control approach," IEEE Trans. Aut.Contr., vol. AC-45, pp. 1951-1969, Nov. 2000 .

[32] P. Arcara and C. Melchiorri, "Control schemes for teleoperation with time delay: a comparative study," Robotics and Autonomous Systems, vol. 38, pp. 4964, Jan. 2002.

[33] K. Hirai and Y. Satoh, "Stability of systems with variable time delay," IEEE Transactions on Automatic Control, vol. AC-25, no. 3, pp. 552-554, 1980.

[34] G. Niemeyer and J.-J. E. Slotine, "Towards force reflecting teleoperation over the Internet," in International Conference on Robotics and Automation, (Leuven, Belgium), pp. 1909-1915, 1998.

[35] N. Chopra, M. W. Spong, S. Hirche, and M. Buss, "Bilateral teleoperation over the internet: the time varying delay problem," in American Control Conference, (Denver, CO), June 2003.

[36] J. H. Park and H. C. Cho, "Sliding mode controller for bilateral teleoperator with time-varying time delay," in Proceedings of the IEEE International Conference on Advanced Intellectual Mechanics, (Atlanta, GA,), pp. 311-316, 1999. 
[37] R. Oboe and P. Fiorini, "A design and control environment for Internet-based teleoperation," International Journal of Robotics Research, vol. 17, no. 4, pp. 433-449, 1998.

[38] R. Oboe, "Force-reflecting teleoperation over the Internet: The JBIT project," Proceedings of the IEEE, vol. 91, no. 3, pp. 449-462, 2003.

[39] I. W. Sandberg, "Some results on the theory of physical systems governed by nonlinear functional equations," Bell Sys. Tech., J., vol. 44, pp. 871-898, 1965.

[40] G. Zames, "On the input-output stability of time-varying nonlinear feedback systems. Part I: Conditions derived using concepts of loop gain, conicity, and positivity," IEEE Transactions on Automatic Control, vol. AC-11, pp. 228238, Apr. 1966.

[41] C. Desoer and M. Vidyasagar, Feedback Systems: Input-Output Properties. Academic Press, 1975.

[42] I.M.Y.Mareels and D.J.Hill, "Monotone stability of nonlinear feedback systems," Journal of Mathematical Systems Estimation and Control, vol. 2, no. 3, pp. 275-291, 1992.

[43] Z.-P. Jiang, A. R. Teel, and L. Praly, "Small-gain theorem for ISS systems and applications," Mathematics of Control, Signals, and Systems, vol. 7, pp. 95120, 1994.

[44] A. R. Teel, "A nonlinear small gain theorem for the analysis of control systems with saturation," IEEE Transactions on Automatic Control, vol. AC-41, pp. 1256-1270, Sept. 1996. 
[45] Z.-P. Jiang, "Control of interconnected nonlinear systems: a small gain viewpoint," in Optimal Control, Stabilization, and Nonsmooth Analysis (M. de Queiroz, M. Malisoff, and P. Wolenski, eds.), pp. 183-195, Heidelberg: Springer-Verlag, 2004.

[46] E. D. Sontag, "Input-to-state stability: Basic concepts and results," in Nonlinear and Optimal Control Theory (P. Nistri and G. Stefani, eds.), pp. 163-220, Berlin: Springer-Verlag, 2006.

[47] A. R. Teel, "Connections between Razumikhin-type theorems and the ISS nonlinear small gain theorem," IEEE Transactions on Automatic Control, vol. AC-43, pp. 960-964, July 1998.

[48] D. Liberzon, "Quantization, time delays, and nonlinear stabilization," IEEE Transactions on Automatic Control, vol. 51, no. 7, pp. 1190-1195, 2006.

[49] G. Enciso and E. D. Sontag, "Global attractivity, I/O monotone small-gain theorems, and biological delay systems," Discrete and Continuous Dynamical Systems, vol. 14, no. 3, pp. 549-578, 2006.

[50] A. R. Teel, D. Nesić, and P. V. Kokotović, "A note on input-to-state stability of sampled-data nonlinear systems," in Proceedings of the 37th IEEE Conference on Decision 8 Control, (Tampa, Florida, USA), pp. 2473-2478, 1998.

[51] D. S. Laila, D. Nesić, and A. R. Teel, "Open and closed loop dissipation inequalities under sampling and controller emulation," European Journal of Control, vol. 18, pp. 109-125, 2002.

[52] D. Nesić, A. R. Teel, and P. V. Kokotović, "Sufficient conditions for stabilization of sampled-data nonlinear systems via discrete-time approximations," Systems \& Control Letters, vol. 38, pp. 259-270, 1999. 
[53] D. Nesić and A. R. Teel, "A framework for stabilization of nonlinear sampleddata systems based on their approximate discrete-time models," IEEE Transactions on Automatic Control, vol. 49, pp. 1103-1122, July 2004.

[54] D. Nesić, A. R. Teel, and E. D. Sontag, "Formulas relating kl stability estimates of discrete-time and sampled-data nonlinear systems," Systems 8 Control Letters, vol. 38, pp. 48-60, 1999.

[55] D. Hristu-Varsakelis and W. S. Levine, eds., Handbook of Networked and Embedded Control Systems. Birkhauser, 2005.

[56] H. K. Khalil, Nonlinear Systems. Upper Saddle River, NJ: Prentice Hall, third ed., 2002.

[57] E. D. Sontag, "Smooth stabilization implies coprime factorization," IEEE Transactions on Automatic Control, vol. AC-34, pp. 435-443, 1989.

[58] J. K. Hale, Theory of Functional Differential Equations. New York: SpringerVerlag, 1977.

[59] D. Nesić and A. R. Teel, "Sampled-data control of nonlinear systems: an overview of recent results," in Perspectives on Robust Control (R.S.O.Moheimani, ed.), pp. 221-239, Springer-Verlag: New York, 2001.

[60] I. G. Polushin, P. X. Liu, and C.-H. Lung, "A control scheme for stable forcereflecting teleoperation over IP networks," IEEE Transactions on Systems, Man, and Cybernetics, Part B: Cybernetics, vol. 36, no. 4, pp. 930-939, 2006.

[61] M. W. Spong, "Motion control of robot manipulators," in Handbook of Control (W. Levine, ed.), pp. 1339-1350, CRC Press, 1996. 
[62] D. Angeli, "Input-to-state stability of PD-controlled robotic systems," Automatica, vol. 35, pp. 1285-1290, 1999.

[63] E. D. Sontag and Y. Wang, "On characterizations of the input-to-state stability property," Systems $\mathcal{E}$ Control Letters, vol. 24, pp. 351-359, 1995.

[64] G. E. Shilov, Elementary Functional Analysis. Cambridge, Mass.: MIT Press, 1974. translated and edited by Richard A. Silverman.

[65] B. Hannaford, "Stability and performance tradeoffs in bi-lateral telemanipulation," in Proceedings of the 1989 IEEE International Conference on Robotics and Automation, pp. 1764-1767, 1989.

[66] K. J. Kuchenbecker and G. Niemeyer, "Induced master motion in forcereflecting teleoperation," ASME Journal of Dynamic Systems, Measurement, and Control, vol. 128, no. 4, pp. 800-810, 2006.

[67] J. E. Speich, K. Fite, and M. Goldfarb, "A method for simultaneously increasing transparency and stability robustness in bilateral telemanipulation," in Proceedings of the IEEE International Conference on Robotics and Automation ICRA 2000, (San Francisco, CA), pp. 2671-2676, Apr. 2000.

[68] B. Hannaford, "A design framework for teleoperators with kinesthetic feedback," IEEE Transactions on Robotics and Automation, vol. 5, pp. 426-434, Aug. 1989.

[69] R. W. Daniel and P. R. McAree, "Fundamental linits of performance for force reflecting teleoperation," International Journal of Robotics Research, vol. 8, pp. 811-830, 1998. 
[70] K. J. Kuchenbecker and G. Niemeyer, "Canceling induced master motion in force-reflecting teleoperation," in Proceedings of ASME International Mechanical Engineering Congress and Exposition IMECE 2004, vol. 2, (Anaheim, CA), Nov. 2004.

[71] E. Burdet, R. Osu, D. W. Franklin, T. E. Milner, and M. Kawato, "The central nervous system stabilizes unstable dynamics by learning optimal impedance," Nature, vol. 414, pp. 446-449, Nov. 2001.

[72] A. Stotsky and I. Kolmanovsky, "Application of input estimation techniques to charge estimation and control in automotive engines," Control Engineering Practice, vol. 10, pp. 1371-1383, 2002.

[73] D. Gutierrez, A. Shah, and D. Harris, "Performance of remote anatomy and surgical training applications under varied network conditions," in Proceedings of World Conference on Educational Multimedia, Hypermedia and Telecommunications 2002, (Denver, Colorado, USA), pp. 662-667, 2002.

[74] P. Dev, D. Harris, D. Gutierrez, A. Shah, and S. Senger, "End-to-end performance measurement of internet based medical applications," in Proceedings of the 2002 American Medical Informatics Association Symposium, (San Antonio, TX), pp. 205-209, Nov. 2002.

[75] F. T. Buzan and T. B. Sheridan, "A model-based predictive operator aid for telemanipulators with time delay," in IEEE International Conference on Systems, Man and Cybernetics, vol. 1, (Cambridge, MA), pp. 138-143, Nov. 1989.

[76] K. Kosuge, K. Takeo, T. Fukuda, T. Sugiura, A. Sakai, and K. Yamada, "Unified approach for teleoperation of virtual and real environment for skill 
based teleoperation," in Proceedings of the IEEE/RSJ/GI International Conference on Intelligent Robots and Systems IROS '94, vol. 2, (Munich, Germany), pp. 1242-1247, Sept. 1994.

[77] K. Kosuge, K. Takeo, and T. Fukuda, "Unified approach for teleoperation of virtual and real environment-manipulation based on reference dynamics," in Proceedings of the 1995 IEEE International Conference on Robotics and Automation, vol. 1, (Nagoya, Aichi, Japan), pp. 938-943, May 1995.

[78] W.-K. Yoon, T. Goshozono, H. Kawabe, M. Kinami, Y. Tsumaki, M. Uchiyama, M. Oda, and T. Doi, "Model-based space robot teleoperation of ETS-VII manipulator," IEEE Transactions on Robotics and Automation, vol. 20, pp. 602-6.12, June 2004.

[79] L. J. Love and W. J. Book, "Force reflecting teleoperation with adaptive impedance control," IEEE Transactions on Systems, Man, and Cybernetics Part B:Cybernetics, vol. 34, pp. 159-166, Feb. 2004.

[80] L. Huijun and S. Aiguo, "Virtual-environment modeling and correction for force-reflecting teleoperation with time delay," IEEE Transactions on Industrial Electronics, vol. 54, pp. 1227-1233, Jan 2007.

[81] P. Mitra and G. Niemeyer, "Model-mediated telemanipulation," The International Journal of Robotics Research, vol. 27, pp. 1837-1843, feb 2008.

[82] P. F. Hokayem and M. W. Spong, "Bilateral teleoperation: A historical survey," Automatica, vol. 42, no. 12, pp. 2035-2057, 2006.

[83] M. Arcak and D. Nesić, "Sampled-data observer design via approximate discrete-time models and emulation," Automatica, vol. 40, no. 11, pp. 1931$1938,2004$. 
[84] I. G. Polushin and H. J. Marquez, "Multirate versions of sampled-data stabilization of nonlinear systems," Automatica, vol. 40, pp. 1035-1041, 2004.

[85] I. G. Polushin, P. X. Liu, and C.-H. Lung, "On the model-based approach to nonlinear networked control systems," Automatica, vol. 44, no. 9, pp. 2409$2414,2008$.

[86] W. Zhang, M. S. Branicky, and S. M. Phillips, "Stability of networked control systems," IEEE Control Systems Magazine, vol. 21, pp. 84-99, Feb. 2001.

[87] G. C. Walsh and H. Ye, "Scheduling of networked control systems," IEEE Control Systems Magazine, vol. 21, pp. 57-65, Feb. 2001.

[88] G. C. Walsh, O. Beldiman, and L. G. Bushnell, "Asymptotic behavior of nonlinear networked control systems," IEEE Transactions on Automatic Control, vol. 46, pp. 1093-1097, July 2001.

[89] G. C. Walsh, H. Ye, and L. G. Bushnell, "Stability analysis of networked control systems," IEEE Transactions on Control Systems Technology, vol. 10, pp. 438-446, May 2002.

[90] H. Ishii and B. A. Francis, Limited Data Rate in Control Systems with Networks. Berlin: Springer-Verlag, 2002.

[91] D. Nesić and A. R. Teel, "Input-output stability properties of networked control systems," IEEE Transactions on Automatic Control, vol. 49, pp. 16501667, Oct. 2004.

[92] P. Naghshtabrizi and J. Hespanha, "Designing an observer-based controller for a network control system," in 44th IEEE Conference on Decision and Con- 
trol and 2005 European Control Conference CDC-ECC '05, (Seville, Spain), pp. 848-853, Dec. 2005.

[93] J. Eidson and W. Cole, "Ethernet rules closed-loop system," InTech, pp. 3942, June 1998.

[94] L. A. Montestruque and P. J. Antsaklis, "On the model-based control of networked control systems," Automatica, vol. 39, pp. 1837-1843, 2004.

[95] L. A. Montestruque and P. J. Antsaklis, "Networked control systems: A model-based approach," in Handbook of Networked and Embedded Control Systems (D. Hristu-Varsakelis and W. S. Levine, eds.), pp. 601-625, Birkhauser Boston, 2005.

[96] R. Luck and A. Ray, "An observer-based compensator for distributed delays," Automatica, vol. 26, no. 5, pp. 903-908, 1990.

[97] H. K. Khalil, "Performance recovery under output feedback sampled-data stabilization of a class of nonlinear systems," IEEE Transactions on Automatic Control, vol. 49, pp. 2173-2184, Dec. 2004.

[98] I. G. Polushin, H. J. Marquez, A. Tayebi, and P. X. Liu, "A multichannel IOS small gain theorem for systems with multiple time-varying communication delays," IEEE Transactions on Automatic Control, vol. 54, no. 1, 2009. to appear.

[99] I. G. Polushin, H. J. Marquez, A. Tayebi, and P. X. Liu, "A multichannel IOS small gain theorem for systems with multiple time-varying communication delays," in 46th IEEE Conference on Decision and Control, (New Orleans, LA), Dec. 2007. 
[100] I. G. Polushin, P. X. Liu, and C.-H. Lung, "A control scheme for stable forcereflecting teleoperation over IP networks," in IEEE/RSJ International Conference on Intelligent Robots and Systems IROS 2005, (Edmonton, AB, Canada), Aug. 2005.

[101] I. G. Polushin, P. X. Liu, C.-H. Lung, and G. D. On, "Position-error based schemes for bilateral teleoperation with time delay: Theory and experiments," in International Conference on Mechatronics and Automation ICMA 2006, (R. P. China), June 2006.

[102] I. G. Polushin, P. X. Liu, and C.-H. Lung, "A force-reflection algorithm for improved transparency in bilateral teleoperation with communication delay," IEEE/ASME Transactions on Mechatronics, vol. 12, no. 3, pp. 361-374, 2007.

[103] I. G. Polushin, P. X. Liu, and C.-H. Lung, "Projection-based force reflection algorithm for stable bilateral teleoperation over networks," IEEE Transactions on Instrumentation and Measurement, vol. 57, no. 9, pp. 1854-1865, 2008.

[104] I. G. Polushin, P. X. Liu, and C.-H. Lung, "A force-reflection algorithm for improved transparency in bilateral teleoperation with communication delay," in 2006 IEEE International Conference on Robotics and Automation, (Orlando, FL), May 2006.

[105] I. G. Polushin, P. X. Liu, and C.-H. Lung, "Projection-based force reflection algorithm for stable bilateral teleoperation over networks," in IEEE/RSJ International Conference on Intelligent Robots and Systems IROS 2007, (San Diego, CA), Oct. 2007.

[106] I. G. Polushin, P. X. Liu, and C.-H. Lung, "Stability of bilateral teleoperators with projection-based force reflection algorithms," in 2008 IEEE International 
References

Conference on Robotics and Automation, (Pasadena, CA), pp. 677-682, May 2008.

[107] I. G. Polushin, P. X. Liu, and C.-H. Lung, "On the model-based approach to nonlinear networked control systems," in American Control Conference, (New York City, NY), July 2007.

[108] I. G. Polushin, J. P. Rhinelander, P. X. Liu, and C.-H. Lung, "A scheme for virtual reality enhanced bilateral teleoperation with time delay," in 2008 IEEE Instrumentation and Measurement Technology Conference, (Victoria, BC), pp. 1819-1822, May 2008.

[109] N. A. Tanner and G. Niemeyer, "Improving perception in time-delayed telerobotics," The International Journal of Robotics Research, vol. 24, pp. 631-644, aug 2005.

[110] R. M. Murray, Z, Li, and S. S. Sastry, A Mathematical Introduction to Robotic Manipulation. CRC Press, 1994.

[111] P. Joice, G. B. Hanna, and A. Cuschieri, "Errors enacted during endoscopic surgery - a human reliability analysis," Applied Ergonomics, vol. 29, pp. 409414, Dec. 1998.

[112] M. Y. Sung, Y. Yoo, K. Jun, N.-J. Kim, and J. Chae, "Experiments for a collaborative haptic virtual reality," in Proceedings of the 40th 16th International Conference on Artificial Reality and Telexistence-Workshops ICAT 06, (Hangzhou, P.R.China), pp. 174-179, Nov. 2006.

[113] J. Kim, H. Kin, B. K. Tay, M. Muniyandi, M. A. Srinivasan, J. Jordan, J. Mortensen, M. Oliveira, and M. Slater, "Transatlantic touch: A study of 
haptic collaboration over long distance," Presence: Teleoperators 6 Virtual Environments, vol. 13, no. 3, pp. 328-337, 2004.

[114] E. Kohler, M. Handley, and S. Floyd, "Designing decp: congestion control without reliability," SIGCOMM Comput. Commun. Rev., vol. 36, no. 4, pp. $27-38,2006$.

[115] S. Kandula, D. Katabi, B. Davie, and A. Charny, "Walking the tightrope: Responsive yet stable traffic engineering," in Proceedings of ACM SIGCOMM'05, (Philadelphia, PA), Aug. 2005. 


\section{Appendix A}

\section{Modelling of the Phantom ${ }^{\mathrm{TM}}$}

\section{Device}

The purpose of this appendix is to present a complete derivation of the kinematic and dynamic models of the two Phantom TM devices used in our experiments. The overall teleoperator system is shown in figure A.1. The master device is equipped with standard gimbal end-effector, while the slave has a marker attached to the end. The master and the slave devices are shown in figures A.2 and A.3, respectively. In the derivation below, we used the approach and the notation adopted in [110]. Majority of the calculations was performed using Maple ${ }^{T M}$ software.

\section{A.1 Forward Kinematics}

The forward kinematics of a manipulator can generally be described as a map $\mathbb{S}^{n} \rightarrow \mathbb{S} E(3)$ that gives the end-effector configuration in terms of joint angles. Here, the end-effector configuration is understood as the configuration (i.e., position and orientation) of the tool frame relative to the based frame. To determine the forward 


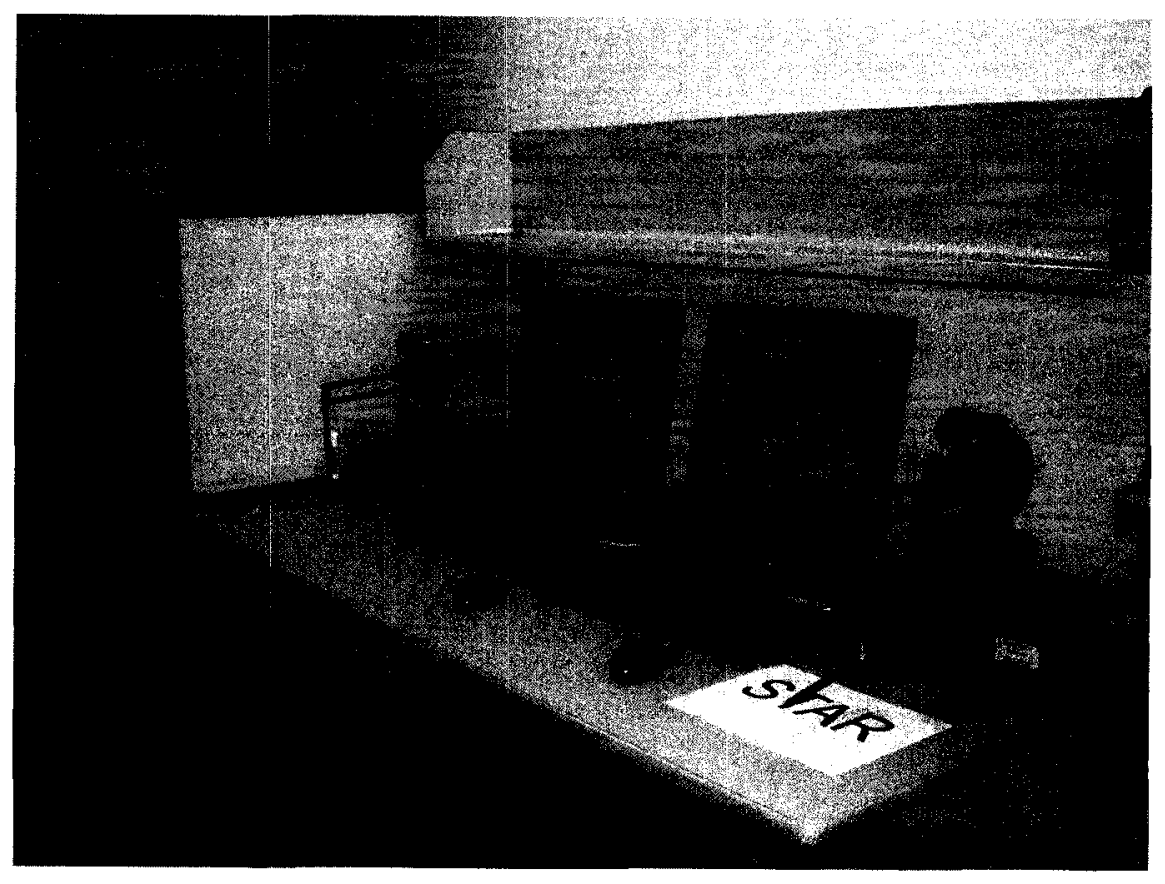

Figure A.1: Teleoperator system

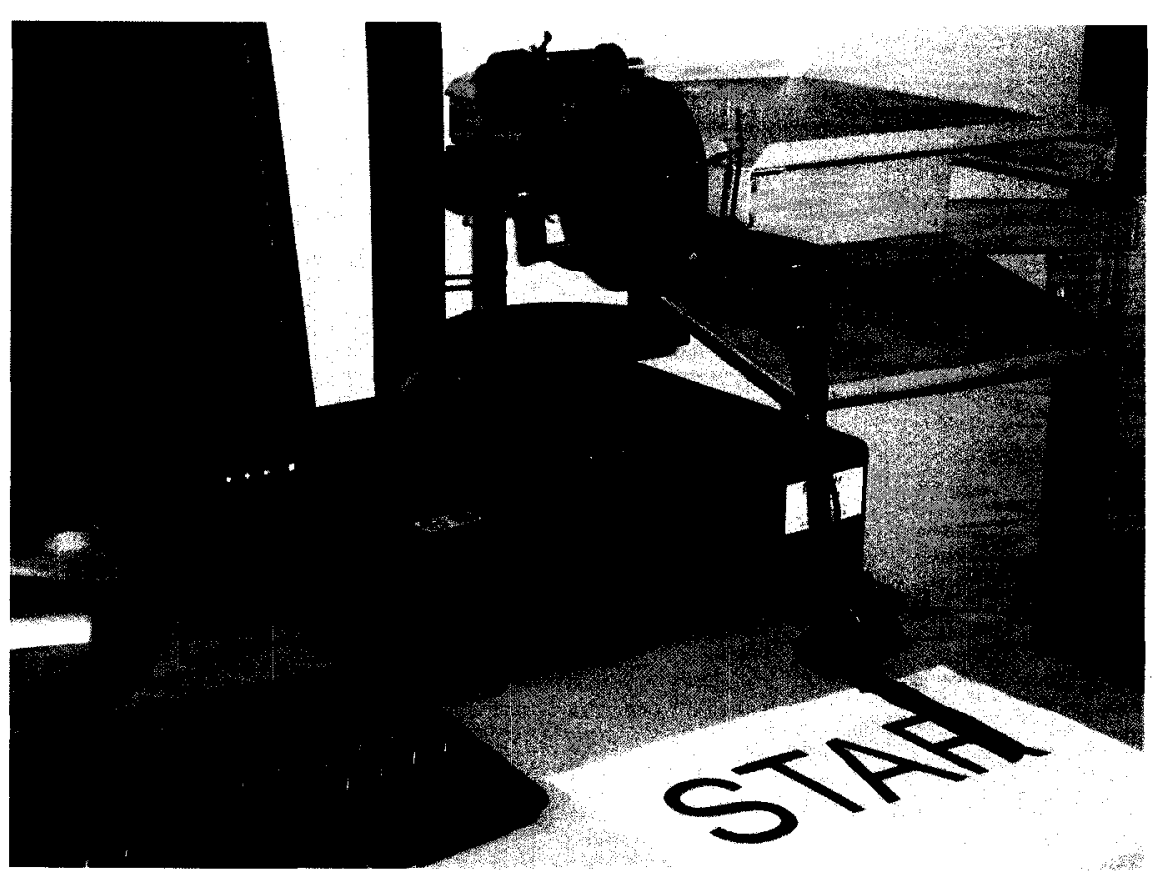

Figure A.2: Master device 


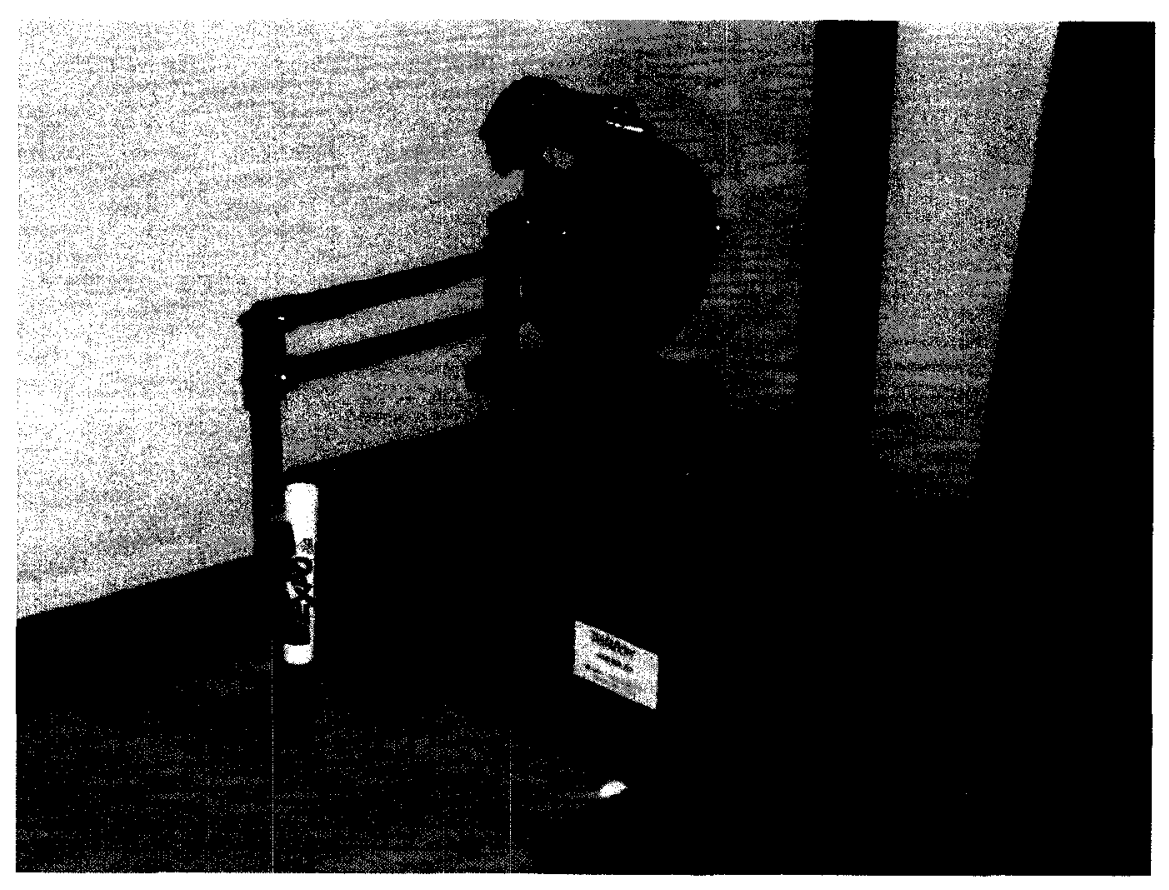

Figure A.3: Slave device
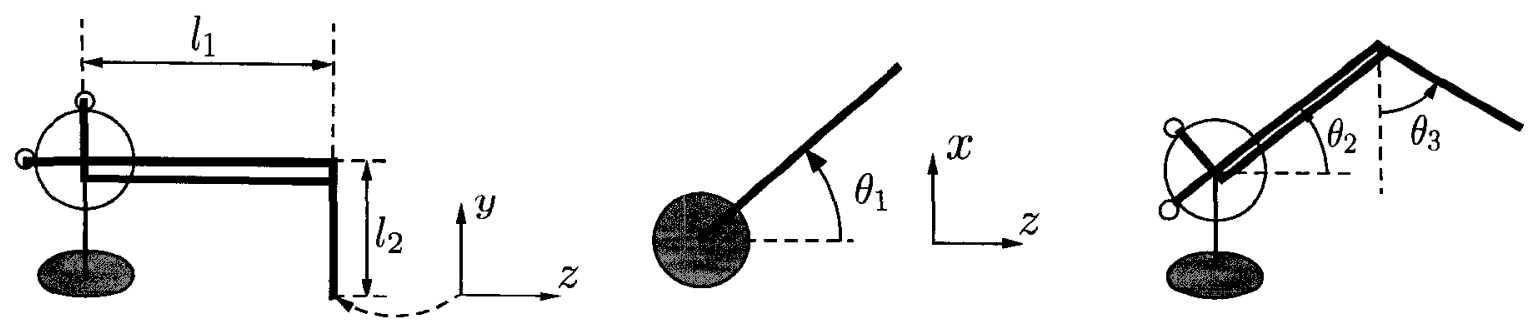

Figure A.4: Kinematic scheme of the Phantom ${ }^{T M}$ device 
kinematics of the Phantom ${ }^{T M}$ device, consider its kinematic scheme shown in Figure A.4. For simplicity of calculations, we choose the base frame to be coincident with the tool frame when $\theta=[0 ; 0 ; 0]$, which implies

$$
g_{s t}(0)=\mathbb{I}_{4 \times 4}
$$

The forward kinematics map $\mathbb{S}^{3} \rightarrow \mathbb{S} E(3)$ of the Phantom ${ }^{T M}$ device is described according to the following product of exponentials formula

$$
g_{s t}(\theta)=e^{\hat{\xi}_{1} \theta_{1}} e^{\hat{\xi}_{2} \theta_{2}} e^{\hat{\xi}_{3}\left(\theta_{3}-\theta_{2}\right)} g_{s t}(0)=e^{\hat{\xi}_{1} \theta_{1}} e^{\hat{\xi}_{2} \theta_{2}} e^{\hat{\xi}_{3}\left(\theta_{3}-\theta_{2}\right)}
$$

To calculate the underlying exponentials, note first that in the initial configuration $\left(\theta_{1}=\theta_{2}=\theta_{3}=0\right)$, the directions of the axes of rotation for $i$-th joints, $i=1,2,3$, are described by vectors $\omega_{i}$, as follows

$$
\omega_{1}=\left[\begin{array}{l}
0 \\
1 \\
0
\end{array}\right], \quad \omega_{2}=\left[\begin{array}{c}
-1 \\
0 \\
0
\end{array}\right], \quad \omega_{3}=\left[\begin{array}{c}
-1 \\
0 \\
0
\end{array}\right],
$$

and the corresponding axes points can be chosen as follows,

$$
q_{1}=\left[\begin{array}{c}
0 \\
0 \\
-l_{1}
\end{array}\right], \quad q_{2}=\left[\begin{array}{c}
0 \\
l_{2} \\
-l_{1}
\end{array}\right], \quad q_{3}=\left[\begin{array}{c}
0 \\
l_{2} \\
0
\end{array}\right] .
$$

Since all the joints are revolute, the corresponding twists $\xi_{i}, i=1,2,3$ are calculated according to the formula

$$
\xi_{i}=\left[\begin{array}{c}
v_{i} \\
\omega_{i}
\end{array}\right]=\left[\begin{array}{c}
-\omega_{i} \times q_{i} \\
\omega_{i}
\end{array}\right] .
$$


Appendix A. Modelling of the Phantom ${ }^{T} M_{\text {Device }}$

Performing the calculations, we get

$$
\xi_{1}=\left[\begin{array}{c}
v_{1} \\
\omega_{1}
\end{array}\right]=\left[\begin{array}{c}
l_{1} \\
0 \\
0 \\
0 \\
1 \\
0
\end{array}\right], \quad \xi_{2}=\left[\begin{array}{c}
v_{2} \\
\omega_{2}
\end{array}\right]=\left[\begin{array}{c}
0 \\
l_{1} \\
l_{2} \\
-1 \\
0 \\
0
\end{array}\right], \quad \xi_{3}=\left[\begin{array}{c}
v_{3} \\
\omega_{3}
\end{array}\right]=\left[\begin{array}{c}
0 \\
0 \\
l_{2} \\
-1 \\
0 \\
0
\end{array}\right] .
$$

Now, the corresponding exponential maps are calculated according to the formula (2.36) from [110], as follows,

$$
e^{\hat{\xi} \theta}=\left[\begin{array}{cc}
e^{\hat{\omega} \theta} & \left(\mathbb{I}-e^{\hat{\omega} \theta}\right)(\omega \times v)+\omega \omega^{T} v \theta \\
0 & 1
\end{array}\right],
$$

which is valid whenever $\omega \neq 0$. Performing calculations according to the above formula, one gets

$$
\begin{gathered}
e^{\hat{\xi}_{1} \theta_{1}}=\left[\begin{array}{cccc}
\cos \theta_{1} & 0 & \sin \theta_{1} & l_{1} \sin \theta_{1} \\
0 & 1 & 0 & 0 \\
-\sin \theta_{1} & 0 & \cos \theta_{1} & \left(\cos \theta_{1}-1\right) l_{1} \\
0 & 0 & 0 & 1
\end{array}\right] \\
e^{\tilde{\xi}_{2} \theta_{2}}=\left[\begin{array}{cccc}
1 & 0 & 0 & 0 \\
0 & \cos \theta_{2} & \sin \theta_{2} & l_{2}\left(1-\cos \theta_{2}\right)+l_{1} \sin \theta_{2} \\
0 & -\sin \theta_{2} & \cos \theta_{2} & l_{2} \sin \theta_{2}+l_{1}\left(\cos \theta_{2}-1\right) \\
0 & 0 & 0 & 1
\end{array}\right], \\
e^{\hat{\xi}_{3} \theta_{3}}=\left[\begin{array}{cccc}
1 & 0 & 0 & 0 \\
0 & \cos \theta_{3} & \sin \theta_{3} & l_{2}\left(1-\cos \theta_{3}\right) \\
0 & -\sin \theta_{3} & \cos \theta_{3} & l_{2} \sin \theta_{3} \\
0 & 0 & 0 & 1
\end{array}\right]
\end{gathered}
$$


Appendix A. Modelling of the Phantom $\overline{T M}$ Device

$$
e^{-\hat{\xi}_{3} \theta_{2}}=\left[\begin{array}{cccc}
1 & 0 & 0 & 0 \\
0 & \cos \left(-\theta_{2}\right) & \sin \left(-\theta_{2}\right) & l_{2}\left(1-\cos \left(-\theta_{2}\right)\right) \\
0 & -\sin \left(-\theta_{2}\right) & \cos \left(-\theta_{2}\right) & l_{2} \sin \left(-\theta_{2}\right) \\
0 & 0 & 0 & 1
\end{array}\right] .
$$

Combining all the above, we get

$$
=\left[\begin{array}{cccc}
g_{s t}\left(\theta_{1}, \theta_{2}, \theta_{3}\right) \\
\cos \theta_{1} & -\sin \theta_{1} \sin \theta_{3} & \sin \theta_{1} \cos \theta_{3} & \sin \theta_{1}\left(l_{2} \sin \theta_{3}+l_{1} \cos \theta_{2}\right) \\
0 & \cos \theta_{3} & \sin \theta_{3} & l_{2}\left(1-\cos \theta_{3}\right)+l_{1} \sin \theta_{2} \\
-\sin \theta_{1} & -\cos \theta_{1} \sin \theta_{3} & \cos \theta_{1} \cos \theta_{3} & -l_{1}+\cos \theta_{1}\left(l_{2} \sin \theta_{3}+l_{1} \cos \theta_{2}\right) \\
0 & 0 & 0 & 1
\end{array}\right],
$$

which represents, in the homogeneous coordinates, the forward kinematics of the Phantom $^{T M}$ device.

\section{A.2 Manipulator Jacobian}

To calculate the spatial Jacobian of the Phantom ${ }^{T M}$ device, we use formula

$$
J^{s}:=\left[\begin{array}{lll}
\xi_{1} & \xi_{2}^{\prime} & \xi_{3}^{\prime}
\end{array}\right]
$$

where $\xi_{i}^{\prime}$ is the $i$-th joint twist transformed to the current manipulator configuration,

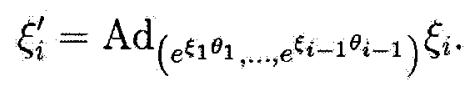

We will calculate $\xi_{i}^{\prime}, i=1,2,3$, according to the formulas

$$
\xi_{i}^{\prime}=\left[\begin{array}{c}
-\omega_{i}^{\prime} \times q_{i}^{\prime} \\
\omega_{i}^{\prime}
\end{array}\right],
$$

where $\omega_{i}^{\prime}$ is the unit vector in the direction of the twist axis, and $q_{i}^{\prime}$ is a point on the axis, both transformed to the current manipulator configuration. By inspection, we 
Appendix A. Modelling of the Phanton ${ }^{T M}$ Device

have

$$
\omega_{1}^{\prime}=\omega_{1}=\left[\begin{array}{l}
0 \\
1 \\
0
\end{array}\right], \quad \omega_{2}^{\prime}=\left[\begin{array}{c}
-\cos \theta_{1} \\
0 \\
\sin \theta_{1}
\end{array}\right], \quad \omega_{3}^{\prime}=\left[\begin{array}{c}
-\cos \theta_{1} \\
0 \\
\sin \theta_{1}
\end{array}\right]
$$

and

$$
q_{1}^{\prime}=q_{1}=\left[\begin{array}{c}
0 \\
0 \\
-l_{1}
\end{array}\right], \quad q_{2}^{\prime}=q_{2}=\left[\begin{array}{c}
0 \\
l_{2} \\
-l_{1}
\end{array}\right], \quad q_{3}^{\prime}=\left[\begin{array}{c}
l_{1} \sin \theta_{1} \cos \theta_{2} \\
l_{2}+l_{1} \sin \theta_{2} \\
l_{1}\left(\cos \theta_{1} \cos \theta_{2}-1\right)
\end{array}\right] .
$$

Using the above formulas, we get

$$
\xi_{1}^{\prime}=\left[\begin{array}{c}
l_{1} \\
0 \\
0 \\
0 \\
1 \\
0
\end{array}\right], \quad \xi_{2}^{\prime}=\left[\begin{array}{c}
l_{2} \sin \theta_{1} \\
l_{1} \cos \theta_{1} \\
l_{2} \cos \theta_{1} \\
-\cos \theta_{1} \\
0 \\
\sin \theta_{1}
\end{array}\right], \quad \xi_{3}^{\prime}=\left[\begin{array}{c}
\sin \theta_{1}\left(l_{2}+l_{1} \sin \theta_{2}\right) \\
l_{1}\left(\cos \theta_{1}-\cos \theta_{2}\right) \\
\cos \theta_{1}\left(l_{2}+l_{1} \sin \theta_{2}\right) \\
-\cos \theta_{1} \\
0 \\
\sin \theta_{1}
\end{array}\right],
$$

and therefore, the spatial Jacobian of the Phantom ${ }^{T M}$ device is as follows,

$$
J^{s}:=\left[\begin{array}{ccc}
l_{1} & l_{2} \sin \theta_{1} & \sin \theta_{1}\left(l_{2}+l_{1} \sin \theta_{2}\right) \\
0 & l_{1} \cos \theta_{1} & l_{1}\left(\cos \theta_{1}-\cos \theta_{2}\right) \\
0 & l_{2} \cos \theta_{1} & \cos \theta_{1}\left(l_{2}+l_{1} \sin \theta_{2}\right) \\
0 & -\cos \theta_{1} & -\cos \theta_{1} \\
1 & 0 & 0 \\
0 & \sin \theta_{1} & \sin \theta_{1}
\end{array}\right] .
$$


Appendix A. Modelling of the Phantom TM Device

\section{A.3 Dynamics}

One possible way to describe the dynamics of a manipulator is in terms of Lagrange's equations with dissipation, as follows

$$
M(\theta) \ddot{\theta}+C(\theta, \dot{\theta}) \dot{\theta}+G(\theta)+K_{F}(\dot{\theta})=\tau .
$$

Here, $M(\theta)$ is the matrix of inertia, $C(\theta, \dot{\theta}) \dot{\theta}$ represents Coriolis and centrifugal torques, $G(\theta)$ and $K_{F}(\dot{\theta})$ are the vectors of gravity torques and dissipative torques, respectively, while $\tau$ is the vector of external torques applied. Below, detailed calculations of the terms that comprise equation (A.2) for Phantom ${ }^{T M}$ device are presented.

First, let us define, for each $i \in\{1,2,3\}$, a coordinate frame $L_{i}$ attached to $i$-th link, such that the origin of $L_{i}$ is put at the $i$-th link's center of mass, and the axes of $L_{i}$ are aligned with the principal inertia axes of $i$-th link, Then, for each $L_{i}$, a forward kinematics map $g_{s l_{i}}(\theta) \in \mathbb{S E}(3)$ can be defined which represents configuration (position and orientation) of the frame $L_{i}$ relative to base frame $S$. In this case, the inertia matrix of the manipulator is calculated according to the formula

$$
M(\theta)=\sum_{i=1}^{3} J_{i}^{T}(\theta) M_{i} J_{i}(\theta),
$$

where $M_{i}$ is the generalized inertia matrix of $i$ - th link written in $L_{i}$ coordinate frame, and $J_{i}(\theta)$ is the body Jacobian corresponding to $g_{s i_{i}}(\theta)$. Since the principal inertia axes of $i$-th link are aligned with the axes of $L_{i}$, the generalized inertia matrices $M_{i}, i=1,2,3$ are diagonal, and have a form

$$
M_{i}=\operatorname{diag}\left\{m_{i}, m_{i}, m_{i}, I_{x i}, I_{y i}, I_{x i}\right\}, \quad, i=1,2,3,
$$

where $m_{i}$ is the mass of the i-th link, and $I_{x i}, I_{y i}, I_{z i}$ are the link's moments of inertia about principal axes. On the other hand, the body Jacobians $J_{i}, i=1,2,3$, 
are calculated according to the formula [110, Formula 3.55]

$$
J_{i}(\theta)=J_{s i_{i}}^{b}(\theta)=\left[\begin{array}{lll}
\xi_{1}^{\dagger} & \xi_{2}^{\dagger} & \xi_{3}^{\dagger}
\end{array}\right]
$$

where

$$
\xi_{j}^{\dagger}=\operatorname{Ad}_{\left(e^{\tilde{\xi}_{j} \theta_{j} \ldots e} e_{i}^{\left.\xi_{i} \theta_{i} g_{s l_{i}}(0)\right)}\right.}^{-1} \xi_{j} \quad \text { for } j \leq i,
$$

and $\xi_{j}^{\dagger}=0$ otherwise $(j>i)$. In the above formula, the inverse adjoint transformation associated with configuration $g \in \mathbb{S} E(3)$,

$$
g=\left[\begin{array}{ll}
R & p \\
0 & 1
\end{array}\right]
$$

is to be calculated by the formula

$$
\operatorname{Ad}_{g}^{-1}=\left[\begin{array}{cc}
R^{T} & -R^{T} \hat{p} \\
0 & R^{T}
\end{array}\right]
$$

The initial configurations $g_{s l_{i}}(0)$ can be found by inspection, as follows

$$
\begin{aligned}
& g_{s l_{1}}(0)=\left[\mathbb{I}\left(\begin{array}{c}
0 \\
r_{1} \\
-l_{1}
\end{array}\right)\right], g_{s l_{2}}(0)=\left[\begin{array}{c}
\mathbb{I}\left(\begin{array}{c}
0 \\
l_{2} \\
-l_{1}+r_{2}
\end{array}\right) \\
1
\end{array}\right] \text {, } \\
& g_{s l_{3}}(0)=\left[\begin{array}{c}
0 \\
\mathbb{I}\left(\begin{array}{c}
0 \\
l_{2}-r_{3} \\
0 \\
0
\end{array}\right)
\end{array}\right]
\end{aligned}
$$

where $r_{1}, r_{2}, r_{3}>0$ are parameters that describe the locations of the centers of 
Appendix A. Modelling of the Phantom ${ }^{T M}$ Device

masses for each link. Intermediate calculations give

$$
\begin{aligned}
& e^{\hat{\xi_{1}} \theta_{1}} g_{s l_{1}}(0)=\left[\begin{array}{cccc}
\cos \theta_{1} & 0 & \sin \theta_{1} & 0 \\
0 & 1 & 0 & r_{1} \\
-\sin \theta_{1} & 0 & \cos \theta_{1} & -l_{1} \\
0 & 0 & 0 & 1
\end{array}\right] \\
& e^{\hat{\varepsilon}_{2} \theta_{2}} g_{s l_{2}}(0)=\left[\begin{array}{cccc}
1 & 0 & 0 & 0 \\
0 & \cos \theta_{2} & \sin \theta_{2} & l_{2}+r_{2} \sin \theta_{2} \\
0 & -\sin \theta_{2} & \cos \theta_{2} & r_{2} \cos \theta_{2}-l_{1} \\
0 & 0 & 0 & 1
\end{array}\right] \\
& e^{\dot{\xi}_{1} \theta_{1}} e^{\hat{\xi}_{2} \theta_{2}} g_{s l_{2}}(0)=\left[\begin{array}{cccc}
\cos \theta_{1} & -\sin \theta_{1} \sin \theta_{2} & \sin \theta_{1} \cos \theta_{2} & r_{2} \sin \theta_{1} \cos \theta_{2} \\
0 & \cos \theta_{2} & \sin \theta_{2} & l_{2}+r_{2} \sin \theta_{2} \\
-\sin \theta_{1} & -\cos \theta_{1} \sin \theta_{2} & \cos \theta_{1} \cos \theta_{2} & r_{2} \cos \theta_{1} \cos \theta_{2}-l_{1} \\
0 & 0 & 0 & 1
\end{array}\right] \text {, } \\
& e^{\hat{\xi}_{3} \theta_{3}} g_{s l_{3}}(0)=\left[\begin{array}{cccc}
1 & 0 & 0 & 0 \\
0 & \cos \theta_{3} & \sin \theta_{3} & l_{2}-r_{3} \cos \theta_{3} \\
0 & -\sin \theta_{3} & \cos \theta_{3} & r_{3} \sin \theta_{3} \\
0 & 0 & 0 & 1
\end{array}\right] \\
& e^{\hat{\xi}_{2} \theta_{2}} e^{\hat{\xi}_{3}\left(\theta_{3}-\theta_{2}\right)} g_{s l_{3}}(0)=\left[\begin{array}{cccc}
1 & 0 & 0 & 0 \\
0 & \cos \theta_{3} & \sin \theta_{3} & -r_{3} \cos \theta_{3}+l_{2}+l_{1} \sin \theta_{2} \\
0 & -\sin \theta_{3} & \cos \theta_{3} & r_{3} \sin \theta_{3}+l_{1}\left(\cos \theta_{2}-1\right) \\
0 & 0 & 0 & 1
\end{array}\right],
\end{aligned}
$$


Appendix A. Modelling of the Phantom ${ }^{T M}$ Device

and

$$
=\left[\begin{array}{cccc}
e^{\hat{\xi}_{1} \theta_{1}} e^{\hat{\xi}_{2} \theta_{2}} e^{\hat{\xi}_{3}\left(\theta_{3}-\theta_{2}\right)} g_{s l_{3}}(0) \\
\cos \theta_{1} & -\sin \theta_{1} \sin \theta_{3} & \sin \theta_{1} \cos \theta_{3} & \sin \theta_{1}\left(r_{3} \sin \theta_{3}+l_{1} \cos \theta_{2}\right) \\
0 & \cos \theta_{3} & \sin \theta_{3} & -r_{3} \cos \theta_{3}+l_{2}+l_{1} \sin \theta_{2} \\
-\sin \theta_{1} & -\cos \theta_{1} \sin \theta_{3} & \cos \theta_{1} \cos \theta_{3} & \cos \theta_{1}\left(r_{3} \sin \theta_{3}+l_{1} \cos \theta_{2}\right)-l_{1} \\
0 & 0 & 0 & 1
\end{array}\right] .
$$

Now, using formulas (A.4)-(A.6), and performing extensive calculations, one gets

$$
\begin{gathered}
J_{1}:=\left[\begin{array}{ccc}
0 & 0 & 0 \\
0 & 0 & 0 \\
0 & 0 & 0 \\
0 & 0 & 0 \\
1 & 0 & 0 \\
0 & 0 & 0
\end{array}\right], J_{2}:=\left[\begin{array}{ccc}
r_{2} \cos \theta_{2} & 0 & 0 \\
0 & r_{2} & 0 \\
0 & 0 & 0 \\
0 & -1 & 0 \\
\cos \theta_{2} & 0 & 0 \\
\sin \theta_{2} & 0 & 0
\end{array}\right], \\
J_{3}:=\left[\begin{array}{ccc}
l_{1} \cos \theta_{2}+r_{3} \sin \theta_{3} \\
0 \\
0 \\
0 \\
\cos \theta_{3} \\
\sin \theta_{3} \\
l_{1} \cos \left(\theta_{3}-\theta_{2}\right) & 0 \\
r_{3}+l_{1} \sin \left(\theta_{3}-\theta_{2}\right) & r_{3} \\
-1 & -1 \\
0 & 0 \\
0 & 0
\end{array}\right],
\end{gathered}
$$

Finally, the matrix of inertia can be calculated according to the formula (A.3), which gives

$$
M(\theta)=\left[\begin{array}{ccc}
M_{11} & 0 & 0 \\
0 & M_{22} & M_{23} \\
0 & M_{32} & M_{33}
\end{array}\right],
$$


where

$$
\begin{aligned}
M_{11}(\theta)= & I_{y 1}+m_{2} r_{2}^{2} \cos ^{2} \theta_{2}+I_{y 2} \cos ^{2} \theta_{2}+I_{z 2} \sin ^{2} \theta_{2}+m_{3} l_{1}^{2} \cos ^{2} \theta_{2} \\
& +m_{3} r_{3}^{2} \sin ^{2} \theta_{3}+2 m_{3} r_{3} l_{1} \cos \theta_{2} \sin \theta_{3}+I_{y 3} \cos ^{2} \theta_{3}+I_{z 3} \sin ^{2} \theta_{3}, \\
M_{22}(\theta)= & m_{2} r_{2}^{2}+I_{x 2}+I_{x 3}+m_{3} l_{1}^{2}+m_{3} r_{3}^{2}-2 m_{3} r_{3} l_{1} \sin \left(\theta_{2}-\theta_{3}\right), \\
M_{23}(\theta)= & M_{32}(\theta)=m_{3} r_{3}^{2}+I_{x 3}-m_{3} r_{3} l_{1} \sin \left(\theta_{2}-\theta_{3}\right), \\
M_{33}(\theta)= & m_{3} r_{3}^{2}+I_{x 3} .
\end{aligned}
$$

Next, the matrix of Coriolis and centrifugal torques is defined as follows,

$$
C_{i j}(\theta, \dot{\theta})=\sum_{k=1}^{3} \Gamma_{i j k} \dot{\theta}_{k},
$$

where $\Gamma_{i j k}$ are Christoffel symbols,

$$
\Gamma_{i j k}=\frac{1}{2}\left(\frac{\partial M_{i j}}{\partial \theta_{k}}+\frac{\partial M_{i k}}{\partial \theta_{j}}-\frac{\partial M_{k j}}{\partial \theta_{i}}\right) .
$$

Using the above formula, one gets

$$
\begin{gathered}
\Gamma_{112}=-m_{2} r_{2}^{2} \cos \theta_{2} \sin \theta_{2}-I_{y 2} \cos \theta_{2} \sin \theta_{2}+I_{z 2} \sin \theta_{2} \cos \theta_{2} \\
-m_{3} l_{1}^{2} \sin \theta_{2} \cos \theta_{2}-m_{3} r_{3} l_{1} \sin \theta_{2} \sin \theta_{3}, \\
\Gamma_{113}=I_{z 3} \sin \theta_{3} \cos \theta_{3}-I_{y 3} \cos \theta_{3} \sin \theta_{3}+m_{3} r_{3} l_{1} \cos \theta_{2} \cos \theta_{3}+m_{3} r_{3}^{2} \sin \theta_{3} \cos \theta_{3}, \\
\Gamma_{121}=\Gamma_{112}, \quad \Gamma_{131}=\Gamma_{113}, \quad \Gamma_{211}=-\Gamma_{112}, \quad \Gamma_{222}=-m_{3} r_{3} l_{1} \cos \left(\theta_{2}-\theta_{3}\right), \\
\Gamma_{223}=-\Gamma_{222}, \quad \Gamma_{232}=\Gamma_{223}, \quad \Gamma_{233}=-m_{3} r_{3} l_{1} \cos \left(\theta_{2}-\theta_{3}\right), \quad \Gamma_{311}=-\Gamma_{113},
\end{gathered}
$$

and the rest of the Christoffel symbols are equal to zero. The elements of matrix $C(\theta, \dot{\theta})$, therefore, are calculated according to (A.7), which gives

$$
\begin{aligned}
C_{11}= & \Gamma_{112} \dot{\theta}_{2}+\Gamma_{113} \dot{\theta}_{3} \\
= & -m_{2} r_{2}^{2} \cos \theta_{2} \sin \theta_{2} \dot{\theta}_{2}-I_{y 2} \cos \theta_{2} \sin \theta_{2} \dot{\theta}_{2}+I_{z 2} \sin \theta_{2} \cos \theta_{2} \dot{\theta}_{2} \\
& -m_{3} l_{1}^{2} \sin \theta_{2} \cos \theta_{2} \dot{\theta}_{2}+I_{z 3} \sin \theta_{3} \cos \theta_{3} \dot{\theta}_{3}-I_{y 3} \cos \theta_{3} \sin \theta_{3} \dot{\theta}_{3} \\
& +m_{3} r_{3} l_{1}\left(\cos \theta_{2} \cos \theta_{3} \dot{\theta}_{3}-\sin \theta_{2} \sin \theta_{3} \dot{\theta}_{2}\right)+m_{3} r_{3}^{2} \sin \theta_{3} \cos \theta_{3} \dot{\theta}_{3},
\end{aligned}
$$


Appendix A. Modelling of the Phantom TM Device

$$
\begin{aligned}
C_{12}= & \Gamma_{121} \dot{\theta}_{1} \\
= & -m_{2} r_{2}^{2} \cos \theta_{2} \sin \theta_{2} \dot{\theta}_{1}-I_{y 2} \cos \theta_{2} \sin \theta_{2} \dot{\theta}_{1}+I_{z 2} \sin \theta_{2} \cos \theta_{2} \dot{\theta}_{1} \\
& -m_{3} l_{1}^{2} \sin \theta_{2} \cos \theta_{2} \dot{\theta}_{1}-m_{3} r_{3} l_{1} \sin \theta_{2} \sin \theta_{3} \dot{\theta}_{1} \\
C_{13}= & \Gamma_{131} \dot{\theta}_{1} \\
= & I_{z 3} \sin \theta_{3} \cos \theta_{3} \dot{\theta}_{1}-I_{y 3} \cos \theta_{3} \sin \theta_{3} \dot{\theta}_{1}+m_{3} r_{3} l_{1} \cos \theta_{2} \cos \theta_{3} \dot{\theta}_{1} \\
& +m_{3} r_{3}^{2} \sin \theta_{3} \cos \theta_{3} \dot{\theta}_{1} \\
C_{21}= & \Gamma_{211} \dot{\theta}_{1}=-\Gamma_{112} \dot{\theta}_{1} \\
= & m_{2} r_{2}^{2} \cos \theta_{2} \sin \theta_{2} \dot{\theta}_{1}+I_{y 2} \cos \theta_{2} \sin \dot{\theta}_{2} \dot{\theta}_{1}-I_{z 2} \sin \theta_{2} \cos \theta_{2} \dot{\theta}_{1} \\
& m_{3} l_{1}^{2} \sin \theta_{2} \cos \theta_{2} \dot{\theta}_{1}+m_{3} r_{3} l_{1} \sin \theta_{2} \sin \theta_{3} \dot{\theta}_{1}, \\
C_{22}= & \Gamma_{222} \dot{\theta}_{2}+\Gamma_{223} \dot{\theta}_{3}=m_{3} r_{3} l_{1} \cos \left(\theta_{2}-\theta_{3}\right)\left(\dot{\theta}_{3}-\dot{\theta}_{2}\right), \\
C_{23}= & \Gamma_{232} \dot{\theta}_{2}+\Gamma_{233} \dot{\theta}_{3}=m_{3} r_{3} l_{1} \cos \left(\theta_{2}-\theta_{3}\right)\left(\dot{\theta}_{2}-\dot{\theta}_{3}\right), \\
C_{31}= & \Gamma_{311} \dot{\theta}_{1} \\
= & -I_{z 3} \sin \theta_{3} \cos \theta_{3} \dot{\theta}_{1}+I_{y 3} \cos \theta_{3} \sin \theta_{3} \dot{\theta}_{1} \\
& -m_{3} r_{3} l_{1} \cos \theta_{2} \cos \theta_{3} \dot{\theta}_{1}-m_{3} r_{3}^{2} \sin \theta_{3} \cos \theta_{3} \dot{\theta}_{1}, \\
C_{32}= & C_{33}=0 .
\end{aligned}
$$

Next, the vector of gravity torques $G(\theta)$ can be calculated according to the formula $G(\theta):=\frac{\partial V}{\partial \theta}$, where $V(\theta)$ is the potential energy of the manipulator. The potential energy of the manipulator is the sum of potential energies of all links,

$$
V(\theta)=m_{1} g h_{1}(\theta)+m_{2} g h_{2}(\theta)+m_{3} g h_{3}(\theta)
$$

Here, $h_{i}$ is the height of the center of the masses for $i$-th link, which can be found using the product of exponentials formula. Performing the calculations, we get

$$
\begin{aligned}
& h_{1}=r_{1}, \\
& h_{2}=l_{2}+r_{2} \sin \theta_{2}, \\
& h_{3}=l_{2}+l_{1} \sin \theta_{2}-r_{3} \cos \theta_{3} .
\end{aligned}
$$


The vector of potential torques, therefore, has a form

$$
G(\theta):=\frac{\partial V}{\partial \theta}=\left[\begin{array}{c}
0 \\
g\left(m_{2} r_{2}+m_{3} l_{1}\right) \cos \theta_{2} \\
g m_{3} r_{3} \sin \theta_{3}
\end{array}\right] .
$$

Finally, the vector of dissipative torques is assumed to have a form

$$
K_{F}(\dot{\theta}):=\left[\begin{array}{l}
k_{V F 1} \dot{\theta}_{1}+k_{C F 1} \operatorname{sign}\left\{\dot{\theta}_{1}\right\} \\
k_{V F 2} \dot{\theta}_{2}+k_{C F 2} \operatorname{sign}\left\{\dot{\theta}_{2}\right\} \\
k_{V F 3} \dot{\theta}_{3}+k_{C F 3} \operatorname{sign}\left\{\dot{\theta}_{3}\right\}
\end{array}\right],
$$

where $k_{V F i}$ and $k_{C F i}, i=1,2,3$, are coefficients of the viscous friction and Coulomb friction, respectively.

\section{A.4 Parameter Identification}

The dynamical model of the Phantom ${ }^{T M}$ device derived in the previous section depends on a number of physical parameters of the manipulator. One possible set of the parameters can be chosen as follows

$$
\begin{gathered}
\pi_{1}=I_{y 1}+I_{y 3}+I_{z 2}, \pi_{2}=I_{y 2}-I_{z 2}+m_{2} r_{2}^{2}+m_{3} l_{1}^{2}, \pi_{3}=I_{z 3}-I_{x 3}-I_{y 3}, \\
\pi_{4}=I_{x 2}+m_{2} r_{2}^{2}+m_{3} l_{1}^{2}, \pi_{5}=m_{3} r_{3}^{2}+I_{x 3}, \pi_{6}=m_{3} r_{3} l_{1}, \pi_{7}=g\left(m_{2} r_{2}+m_{3} l_{1}\right), \\
\pi_{8}=g m_{3} r_{3}, \pi_{9}=k_{V F 1}, \pi_{10}=k_{V F 2}, \pi_{11}=k_{V F 3}, \pi_{12}=k_{C F 1}, \pi_{13}=k_{C F 2}, \pi_{14}=k_{C F 3} .
\end{gathered}
$$

Using the above defined set of parameters, the dynamics of the Phantom ${ }^{T M}$ device can be written in the form

$$
\phi^{T} \pi=\tau,
$$


where $\phi \in \mathbb{R}^{14 \times 3}$ is the regressor matrix, which is defined as follows

$\phi=\left[\begin{array}{ccc}\ddot{\theta}_{1} & 0 & 0 \\ \cos ^{2} \theta_{2} \ddot{\theta}_{1}-2 \cos \theta_{2} \sin \theta_{2} \dot{\theta}_{1} \dot{\theta}_{2} & \cos \theta_{2} \sin \theta_{2} \dot{\theta}_{1}^{2} & 0 \\ \sin ^{2} \theta_{3} \ddot{\theta}_{1}+2 \sin \theta_{3} \cos \theta_{3} \dot{\theta}_{1} \dot{\theta}_{3} & 0 & -\sin \theta_{3} \cos \theta_{3} \dot{\theta}_{1}^{2} \\ 0 & \ddot{\theta}_{2} & 0 \\ \sin ^{2} \theta_{3} \ddot{\theta}_{1}+2 \sin \theta_{3} \cos \theta_{3} \dot{\theta}_{1} \dot{\theta}_{3} & \ddot{\theta}_{2}+\ddot{\theta}_{3} & \ddot{\theta}_{2}+\ddot{\theta}_{3}-\sin \theta_{3} \cos \theta_{3} \dot{\theta}_{1}^{2} \\ 2 \cos \theta_{2} \sin \theta_{3} \ddot{\theta}_{1} & -2 \sin \left(\theta_{2}-\theta_{3}\right) \ddot{\theta}_{2} & -\sin \left(\theta_{2}-\theta_{3}\right) \ddot{\theta}_{2} \\ -2 \sin \theta_{2} \sin \theta_{3} \dot{\theta}_{1} \dot{\theta}_{2} & -\sin \left(\theta_{2}-\theta_{3}\right) \ddot{\theta}_{3} & -\cos \theta_{2} \cos \theta_{3} \dot{\theta}_{1}^{2} \\ +2 \cos \theta_{2} \cos \theta_{3} \dot{\theta}_{1} \dot{\theta}_{3} & +\sin \theta_{2} \sin \theta_{3} \dot{\theta}_{1}^{2} & \\ 0 & \cos \left(\theta_{2}-\theta_{3}\right)\left(\dot{\theta}_{2}-\dot{\theta}_{3}\right)^{2} & 0 \\ 0 & \cos \theta_{2} & \sin \theta_{3} \\ \dot{\theta}_{1} & 0 & 0 \\ 0 & 0 & 0 \\ 0 & \dot{\theta}_{2} & \dot{\theta}_{3} \\ \operatorname{sign}\left\{\dot{\theta}_{1}\right\} & 0 & 0 \\ 0 & 0 & 0 \\ 0 & \operatorname{sign}\left\{\dot{\theta}_{2}\right\} & \left.\dot{\theta}_{3}\right\}\end{array}\right]$

The parameter identification procedure was performed for both the master and the slave devices shown in Figures A.2 and A.3, respectively. In order to perform this procedure, both the manipulators were controlled to move along some sample trajectories that consist of a significant number of sinusoidal signals with different amplitudes and frequencies; the resulting joint positions trajectories as well as joint torques were recorded. To obtain estimates of the corresponding joint velocities and joint accelerations, the trajectories were polynomially approximated locally around each point of interest. Consequently, the least-squares parameter identification procedure was performed; the final estimate of each parameter was obtained by 
averaging all the estimates available and rounding the average to the closest integer number.

The least-squares parameter identification procedure used in our experiments can be described as follows. Suppose, experimental data are available that allows to give the values of the regressor matrix for $N$ different observation instants. Let the value of $\phi$ during $i$-th observation instant be denoted by $\phi(i), i \in\{1, \ldots, N\}$. Let $\phi_{1}(i), \phi_{2}(i), \phi_{3}(i) \in \mathbb{R}^{14}$ be the columns of regressor $\phi(i)$ for $i$-th observation instant. Denote

$$
\Phi_{j}=\left[\begin{array}{c}
\phi_{j}^{T}(1) \\
\phi_{j}^{T}(2) \\
\cdots \\
\phi_{j}^{T}(N)
\end{array}\right] \in \mathbb{R}^{N \times 14}, \quad \Phi=\left[\begin{array}{c}
\Phi_{1} \\
\Phi_{2} \\
\Phi_{3}
\end{array}\right] \in \mathbb{R}^{3 N \times 14}
$$

Analogously, let $\tau_{j}(i)$ be the torque in $j$-th joint measured during $i$-th observation instant. Denote

$$
\mathcal{T}_{j}=\left[\begin{array}{c}
\tau_{j}(1) \\
\tau_{j}(2) \\
\ldots \\
\tau_{j}(N)
\end{array}\right] \in \mathbb{R}^{N}, \quad \mathcal{T}=\left[\begin{array}{c}
\mathcal{T}_{1} \\
\mathcal{T}_{2} \\
\mathcal{T}_{3}
\end{array}\right] \in \mathbb{R}^{3 N}
$$

Then, the parameter estimate $\hat{\pi} \in \mathbb{R}^{14}$ can be calculated according to the leastsquares principle, as follows

$$
\hat{\pi}=\left(\Phi^{T} \Phi\right)^{-1} \Phi^{T} \mathcal{T}
$$

Below, the Matlab ${ }^{T M}$ script is presented which is used to perform the identification procedure.

\%atlab script for robot parameter identification

step $=0.015$; 
$\mathrm{n}=4 ; \%(2 \mathrm{n}+1)$ - number of points for trajectory estimation $n t=3 ; \%(2 n t+1)$ - number of points for torque approximation $\mathrm{np}=2 ; \%$ order of the polynomial approximation of trajectories npt $=1 ; \%$ order of the torque approximation lowerlimit $=100$; upperlimit $=4000$;

for $i=$ lowerlimit:upperlimit

$\mathrm{h}=[-\mathrm{n}: \mathrm{n}]$;

$\mathrm{ht}=[-\mathrm{nt}: \mathrm{nt}]$;

$t t=s t e p * h^{\prime} ;$

ttt=step*ht';

$h i=[i-n: i+n]$;

hit $=[i-n t: i+n t]$

jp1=[jointpositions $(\mathrm{hi}, 1)]$; \%positions of 1st joint

jp2=[jointpositions $(h i, 2)]$; \%position of 2nd joint

jp3 $=[$ jointpositions $(h i, 3)]$; \%position of 3rd joint

jt $1=[$ jointtorques(hit, 1$)] ; \%$ torques of 1 st joint

jt2 $=[$ jointtorques (hit,2)]; \%torques of 2 nd joint

jt3 $=[$ jointtorques (hit, 3$)] ; \%$ torques of 3 rd joint

$\mathrm{p} 1(:, i)=\operatorname{polyfit}(\mathrm{tt}, \mathrm{jp} 1, \mathrm{np})$;

$\mathrm{p} 2(:, i)=\operatorname{polyfit}(\mathrm{tt}, \mathrm{jp} 2, \mathrm{np})$;

$\mathrm{p} 3(:, i)=$ polyfit $(t t, j p 3, n p)$;

t1 $(:, i)=\operatorname{polyfit}(t t t, j t 1, \mathrm{npt})$;

t2 $(:, i)=\operatorname{polyfit}(t t t, j t 2, n p t)$;

t3 $(:, i)=\operatorname{polyfit}(t t t, j t 3, n p t)$;

end

position1st=p1 $(\mathrm{np}+1, \mathrm{)})$;

position $2 n d=p 2(n p+1,:)$; 
Appendix A. Modelling of the Phantom ${ }^{T M}$ Device

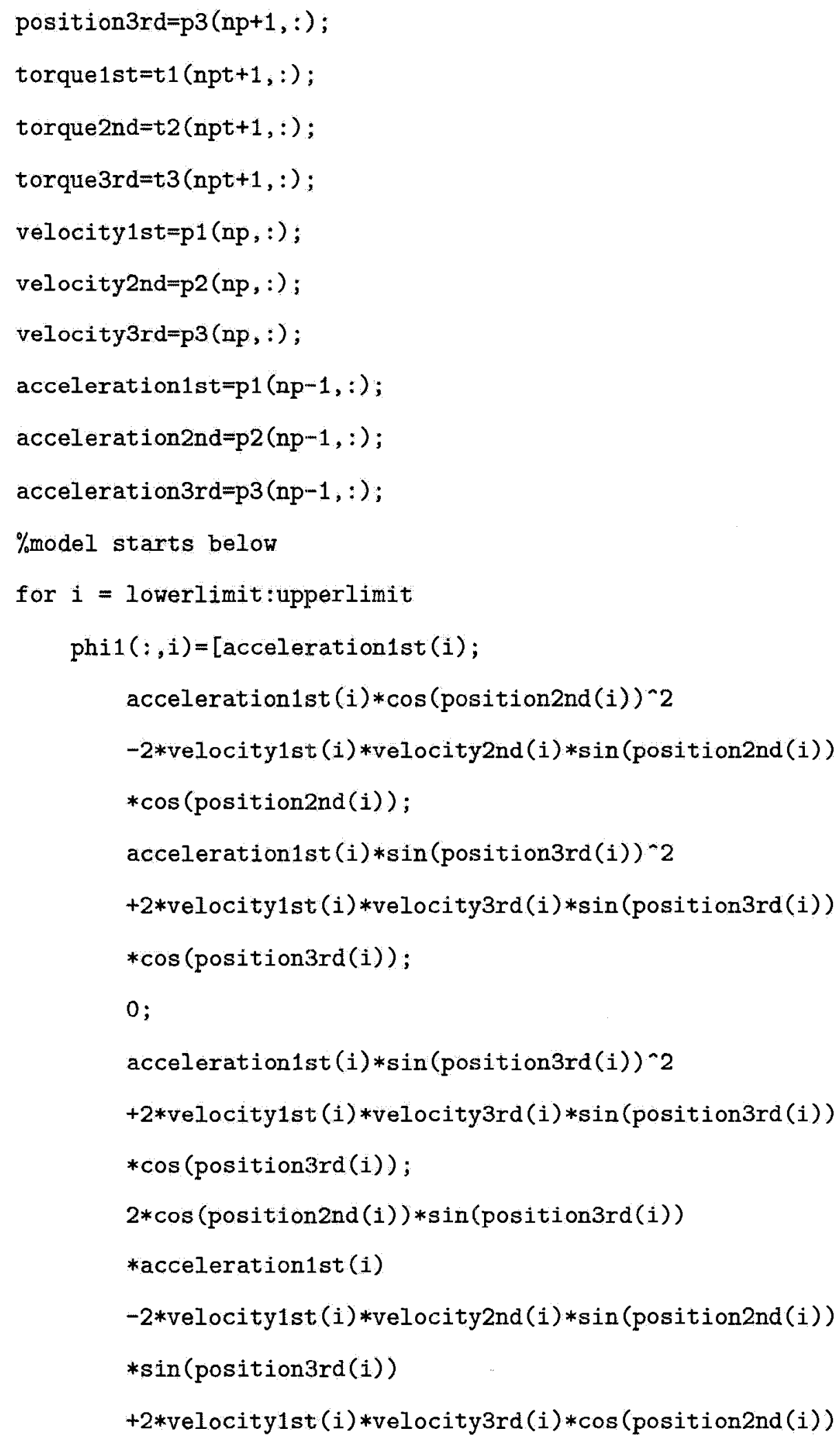


Appendix A. Modelling of the Phantom ${ }^{T M}$ Device

* $\cos ($ position3rd (i));

$0 ; 0$; velocity1st(i); $0 ; 0$; sign(velocity1st(i));0;0]; $\operatorname{phi2}(:, i)=[0$;

velocity1st (i) $2 * \sin ($ position 2 nd (i))* $\cos ($ position 2 nd (i));

0 ;

acceleration2nd(i);

acceleration 2 nd (i) +acceleration $3 r d(i)$;

$-2 * \sin ($ position2nd (i)-position3rd(i))*acceleration2nd (i)

-sin(position2nd(i)-position3rd(i))*acceleration3rd(i)

$+\sin ($ position2nd(i))*sin(position $3 r d(i)) * v e l o c i t y 1 s t(i)-2$

- $\cos ($ position2nd(i)-position3rd(i))*

(velocity2nd(i)-velocity3rd(i)) 2 ;

$\cos ($ position $2 n d(i)) ; 0 ; 0$; velocity2nd(i); $0 ; 0$;

sign (velocity2nd(i)); 0];

$\operatorname{phi} 3(:, i)=[0 ; 0$;

-velocity1st (i)*velocity1st(i)*sin (position3rd(i))

$* \cos ($ position $3 r d(i))$;

0 ; acceleration2nd(i)+acceleration3rd(i)

-velocity1st(i)*velocity1st(i)*sin(position3rd(i))

*cos (position3rd(i));

-sin(position2nd(i)-position3rd(i))*acceleration2nd(i)

-velocity1st (i)*velocity1st (i)*cos (position2nd(i))

* cos (position $3 r d(i))$;

$0 ; \sin (\operatorname{position} 3 \operatorname{rd}(i)) ; 0 ; 0$; velocity3rd(i);0;

$0 ; \operatorname{sign}(v e l o c i t y 3 r d(i))$

]

end

PHI=[phi1'; phi2'; phi3']; 
Appendix A. Modelling of the Phantom ${ }^{T M}$ Device

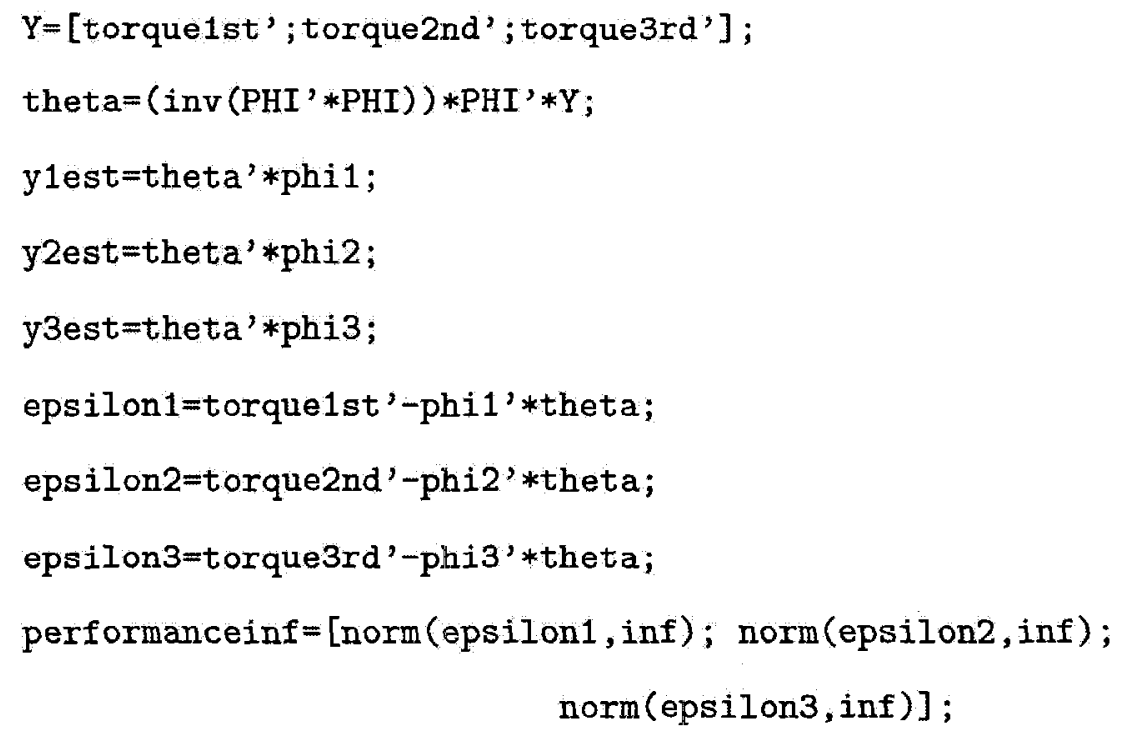

As a result of the above described procedure, in the case of master manipulator, the following parameter estimates are obtained: $\pi_{1}=50, \pi_{2}=60, \pi_{3}=48, \pi_{4}=80$, $\pi_{5}=9, \pi_{6}=8, \pi_{7}=235, \pi_{8}=270, \pi_{9}=25, \pi_{10}=22, \pi_{11}=110, \pi_{12}=82$, $\pi_{13}=160, \pi_{14}=90$. For the slave manipulator, the parameter estimates are as follows: $\pi_{1}=32, \pi_{2}=34, \pi_{3}=20, \pi_{4}=74, \pi_{5}=1, \pi_{6}=2, \pi_{7}=-926, \pi_{8}=-685$, $\pi_{9}=15, \pi_{10}=15, \pi_{11}=90, \pi_{12}=80, \pi_{13}=112, \pi_{14}=55$.

The elements of matrices $M(\theta), C(\theta, \dot{\theta})$, and $G(\theta)$ written in terms of parameters $\pi$ have a form

$$
\begin{aligned}
M_{11}(\theta) & =\pi_{1}+\pi_{2} \cos ^{2} \theta_{2}+\left(\pi_{3}+\pi_{5}\right) \sin ^{2} \theta_{3}+2 \pi_{6} \cos \theta_{2} \sin \theta_{3}, \\
M_{22}(\theta) & =\pi_{4}+\pi_{5}-2 \pi_{6} \sin \left(\theta_{2}-\theta_{3}\right), \\
M_{23}(\theta)=M_{32}(\theta) & =\pi_{5}-\pi_{6} \sin \left(\theta_{2}-\theta_{3}\right), \\
M_{33}(\theta) & =\pi_{5} .
\end{aligned}
$$




$$
\begin{aligned}
C_{11}= & \Gamma_{112} \dot{\theta}_{2}+\Gamma_{113} \dot{\theta}_{3} \\
= & -\left(\pi_{2} \sin \theta_{2} \cos \theta_{2}+\pi_{6} \sin \theta_{2} \sin \theta_{3}\right) \dot{\theta}_{2} \\
& +\left(\left(\pi_{3}+\pi_{5}\right) \sin \theta_{3} \cos \theta_{3}+\pi_{6} \cos \theta_{2} \cos \theta_{3}\right) \dot{\theta}_{3}, \\
C_{12}= & \Gamma_{121} \dot{\theta}_{1}=-\left(\pi_{2} \sin \theta_{2} \cos \theta_{2}+\pi_{6} \sin \theta_{2} \sin \theta_{3}\right) \dot{\theta}_{1}, \\
C_{13}= & \Gamma_{131} \dot{\theta}_{1}=\left(\left(\pi_{3}+\pi_{5}\right) \sin \theta_{3} \cos \theta_{3}+\pi_{6} \cos \theta_{2} \cos \theta_{3}\right) \dot{\theta}_{1}, \\
C_{21}= & \Gamma_{211} \dot{\theta}_{1}=-\Gamma_{112} \dot{\theta}_{1}=\left(\pi_{2} \sin \theta_{2} \cos \theta_{2}+\pi_{6} \sin \theta_{2} \sin \theta_{3}\right) \dot{\theta}_{1}, \\
C_{22}= & \Gamma_{222} \dot{\theta}_{2}+\Gamma_{223} \dot{\theta}_{3}=\pi_{6} \cos \left(\theta_{2}-\theta_{3}\right)\left(\dot{\theta}_{3}-\dot{\theta}_{2}\right) \\
C_{23}= & \Gamma_{232} \dot{\theta}_{2}+\Gamma_{233} \dot{\theta}_{3}=\pi_{6} \cos \left(\theta_{2}-\theta_{3}\right)\left(\dot{\theta}_{2}-\dot{\theta}_{3}\right) \\
C_{31}= & \Gamma_{311} \dot{\theta}_{1}=-\left(\pi_{3}+\pi_{5}\right) \sin \theta_{3} \cos \theta_{3} \dot{\theta}_{1}-\pi_{6} \cos \theta_{2} \cos \theta_{3} \dot{\theta}_{1}, \\
C_{32}= & C_{33}=0 . \\
& G_{1}=0, \quad G_{2}=\pi_{7} \cos \theta_{2}, \quad G_{3}=\pi_{8} \sin \theta_{3} .
\end{aligned}
$$

Finally, the derivatives of inertia matrix $M(\theta)$ which are used in Section 4.6 for the design of input observer can be calculated as follows,

$$
\begin{aligned}
\dot{M}_{11}= & -2 \pi_{2} \cos \theta_{2} \sin \theta_{2} \dot{\theta}_{2}+2\left(\pi_{3}+\pi_{5}\right) \sin \theta_{3} \cos \theta_{3} \dot{\theta}_{3} \\
& +2 \pi_{6}\left(-\sin \theta_{2} \sin \theta_{3} \dot{\theta}_{2}+\cos \theta_{2} \cos \theta_{3} \dot{\theta}_{3}\right), \\
\dot{M}_{22}= & -2 \pi_{6} \cos \left(\theta_{2}-\theta_{3}\right)\left(\dot{\theta}_{2}-\dot{\theta}_{3}\right), \\
\dot{M}_{23}= & \dot{M}_{32}= \\
\dot{M}_{33}= & -\pi_{6} \cos \left(\theta_{2}-\theta_{3}\right)\left(\dot{\theta}_{2}-\dot{\theta}_{3}\right),
\end{aligned}
$$




\section{Appendix B}

\section{Communication Aspects of}

\section{Telesurgery Over The Internet}

In this Appendix, we briefly present some observations related to communication aspects of telesurgery over the Internet. The first trans-oceanic telerobotic surgery, entitled Operation Lindbergh, were performed in 2001 [2; 3]. A group of surgeons located in New York City performed a laparoscopic cholecystectomy on a patient in Strasbourg, France. During this surgery, the communication between two parts of telerobot was performed over dedicated virtual ATM circuit with reserved bandwidth of $10 \mathrm{Mb} / \mathrm{s}, 70 \%$ of which was allocated for transporting the high-quality video image. Across the round-trip distance of $14,000 \mathrm{~km}$, the ATM technology allows to achieve round-trip transport delay approximately equal to $80 \mathrm{~ms}$ with almost zero packet loss. Overall RTT, including video coding/decoding and Ethernet-ATM packet conversion, was achieved approximately equal to $155 \mathrm{~ms}$. "Though we have demonstrated the concept and feasibility of remote telesurgery, we have done so using quite extensive (and expensive) telecommunications resources. It is realized that the demonstration as conducted in operation Lindbergh does not represent a 
cost-effective approach, nor does it constitute a viable scenario" [3]. In terms of communication resources, performing telesurgery over the Internet rather than over dedicated channels would greatly increase its accessibility and decrease cost.

\section{B.1 Visual and Haptic Feedback in Telesurgery}

In $[9 ; 10]$, the roles of the visual and the haptic (force) feedback in applications related to minimally invasive (laparoscopic) surgery were evaluated experimentally. In the experiments presented, tissue samples with different hardness characteristics were grasped with a specially designed laparoscopic grasper, and the subjects were asked to rank these samples from softest to hardest based on visual information, force feedback, or combination of the both. During experiments, subject opened and closed the jaws of the grasper using keyboard. The visual feedback was provided through video screen where subject was able to view the deformation of the samples. On the other hand, the force feedback were received through interaction with the PHANToM device. The experiments clearly show that visual and force feedback used simultaneously result in better performance comparing to the cases where visual feedback as well as force feedback were employed separately. (The other author's hypotheses was that purely force feedback would provide better performance comparing to purely visual feedback; it was not validated satisfactory). It is noted [111], that inappropriate and excessive application of force is a main cause of perforation of a gallbladder. 
Appendix B. Communication Aspects of Telesurgery Over The Internet

\section{B.2 Comparison of QoS Requirements for Visual and Haptic Data}

Quality of Service (QoS) requirements for both remote video and haptic information for surgical training applications were addressed in [73; 74]. More precisely, a remote stereo visual system and a haptic application were compared in terms of the following QoS requirements: i) bandwidth, ii) packet loss percentage, iii) time delay; and iv) jitter, i.e., statistical variance of the delay. It is interesting to note that the video and the haptic application have found to have very different QoS requirements. In particular, the visual information generally requires high bandwidth ( $\geq 40 \mathrm{Mbps}$ ), and is sensitive to packet losses (packet loss rate must be $<0.01 \%$ ), however, it is tolerant enough to time delays (admit up to $0.2 \mathrm{~s}$ of round-trip time (RTT) delay) and is not sensitive to jitter. The haptic information, on the other hand, is more sensitive to delays (it admits up to $40 \mathrm{~ms}$ of RTT delay for abrupt movements and up to $160 \mathrm{~ms}$ RTT for gentle movements), and is extremely sensitive to jitter (must be less than $1 \mathrm{~ms}$ ). However, it is very resilient to packet losses (admit up to $10 \%$ of packet loss rate). It is also claimed in $[73 ; 74]$, that the haptic information requires a significantly lower bandwidth comparing to video ( $\geq 128 \mathrm{kbps}$ ). However, the bandwidth requirements may be different for different type of objects. According to [112], recommended update rates are $1 \mathrm{kHz}$ for a rigid surface and $5-10 \mathrm{kHz}$ for a textured surface, while for transformable objects update rate should be kept as high as possible. Thus, to provide faithful haptic information of deformable objects, significant enough bandwidth may also be required.

One can conclude from the data presented above that the QoS requirements for visual and haptic communications in telesurgical applications differ significantly from each other. Roughly speaking, haptic information is extremely sensitive to 
communication delay and has almost zero tolerance to jitter, but requires comparatively lower bandwidth and is very resilient to packet losses. Video, on the other hand, does require high bandwidth and low packet loss rate, however, it admits higher time delays and is relatively insensitive to jitter. This difference in QoS requirements lead us to a hypotheses that data communication in telesurgery can be decomposed into separate video and haptics communication subproblems. More precisely, these two type of signals can possibly be sent over different connections using different type of communication protocols that "fit" the respective QoS requirements.

\section{B.3 Protocols for Haptic Communication in the Internet}

\section{B.3.1 TCP and UDP}

It was found experimentally $[113 ; 27]$, that TCP is inadequate to support user interactions with the remote objects (environment, virtual environment, or another user) successfully. On the other hand, UDP gives a workable solution. However, it was observed [112] that neither TCP nor UDP satisfy QoS (Quality of Service) requirements of the haptic transmission.

\section{Why UDP provides better solution than TCP?}

- Haptic feedback is extremely delay sensitive. Both UDP and TCP do not provide any delay guarantees. However, TCP implements reliable transmission, in particular, it retransmit lost/corrupted packets, which imply additional delays. 
- Haptic applications would generally prefer timeliness to reliability - at any given moment the transmission of new packet is better than retransmission of the old one. However, TCP can not be told to forget old data.

- Haptic applications are loss-tolerant (packet loss rate $\leq 10 \%$ is admissible) no immediate need in reliable transport service provided by TCP.

- Neither UDP nor TCP provides guarantees on transmission rate. However, TCP implements AIMD congestion control algorithm which has the following features: i) AI part of the algorithm increases sending rate until the point of congestion, which implies that TCP artificially creates congestions from time to time; ii) when congestion occurs, MD quickly decreases sending rate, while AI part acquires bandwidth significantly slower. Thus, sending rate is generally lower for TCP comparing to UDP.

\section{Why UDP is not good enough?}

- In terms of routing mechanisms, UDP relies on Internet's routing protocols such as BGP. In particular, solutions provided by Internet's routing protocols do not necessary lead to the shortest possible round-trip time delay.

- IP does not support (on-line) traffic engineering.

- UDP does not have any congestion control/congestion avoidance mechanism, therefore, occurrence of congestion may imply significant delays.

\section{What functionality can be added?}

- Minimum-Delay Routing

- On-line Traffic Engineering (such as TeXCP); 
Appendix B. Communication Aspects of Telesurgery Over The Internet

- Possibly congestion control:

- Congestion control is a part of TeXCP that guarantees stability of the overall TE scheme.

- Example: DCCP - congestion control without reliability [114].

\section{B.3.2 Example: TeXCP for Haptic Data}

$\mathrm{TeXCP}$ is an on-line distributed TE protocol that combines quick reaction to real time traffic demands with good stability/convergence properties [115]. TeXCP uses multiple precomputed paths to deliver traffic from ingress to egress router, and adaptively moves traffic from over-utilized to underutilized paths responding to real time traffic demands and conditions. To each ingress-egress pair, a TeXCP agent is assigned which is located in the ingress router and uses multiple precomputed paths to deliver traffic from the ingress to the egress. The TeXCP agent uses explicit feedback from the routers to discover path utilization, and adaptively moves traffic from over-utilized to underutilized paths using the load balancer. To guarantee quick reaction and absence of overshoots, oscillations, and instability, a light weight perpath XCP-like controller is utilized. Below, we consider some of the main TeXCP components, and address modifications that can be made to these components in order to improve the communications of haptic data.

\section{Path Selection}

The ISP preconfigures TeXCP with a set of paths between each ingress-egress (IE) pair. The choice of these paths is based solely on topology and is independent of the state of each path. By default, TeXCP picks the shortest paths, where the path length is set to its propagation delay. 


\section{Probing Network State}

In order to keep track of the utilization of each path, a TeXCP agent maintains a probe timer which fires every $T_{p}$ seconds. Every time when the timer fires, the TeXCP agent sends probes to each path to learn maximal utilization along the path. A router along the path compares the utilization reported in the packet with the utilization of its output link. If the utilization reported in the packet is smaller than the utilization of the router's output link, the router overwrites utilization in the packet with the new value. The egress node sends the content of the probe packet back to ingress node with the packet acknowledgment, thus informing the TeXCP agent about utilization along the path.

Probe loss is an indication of failure along the path. If the probe is not acknowledged, the TeXCP agent exponentially increases it's estimate of the corresponding path utilization. As a result, failed and highly congested paths are quickly recognized.

- In addition to the utilization of each path, the current single-trip time (STT) delay of each path can be determined.

\section{The Load Balancer}

The load balancer (LB) balances the traffic between available ingress-egress paths in order to keep the maximal utilization of the network as low as possible.

- The load balancer may additionally rebalance the haptic data flows between available paths taking into account the STT delay of each path. 


\section{B.4 Conclusions}

Performing telesurgery over the Internet rather than over dedicated channels would greatly increase its accessibility and decrease cost. It was shown experimentally that, in telesurgical systems, visual and force feedback used simultaneously result in better performance comparing to the cases where visual feedback as well as force feedback were employed separately. It was observed, however, that the video and haptic information have very different QoS requirements. This implies that the video and the haptics signals can possibly be sent over different connections using different type of communication protocols that "fit" the respective QoS requirements. In the Internet-based telesurgery systems, the communication of the haptic data can probably be performed using UDP-like unreliable communication protocols with some additional features that may include minimum-delay routing, on-line traffic engineering, and possibly congestion control. 